\title{
Biophysical and biochemical studies of the outer membrane proteins OprG and OprH from \\ Pseudomonas aeruginosa
}

\author{
Iga Kucharska \\ Zgorzelec, Poland
}

M.S., Wroclaw University of Technology, 2011

A Dissertation presented to the Graduate Faculty of the University of Virginia in Candidacy for the Degree of Doctor of Philosophy

Department of Molecular Physiology and Biological Physics

University of Virginia

August, 2016 


\section{Abstract}

Pseudomonas aeruginosa is a common Gram-negative bacterium that can be found in many different environments. It is also an opportunistic human pathogen and the most common cause of lung infections in cystic fibrosis patients. $P$. aeruginosa infections are very challenging to treat as this bacterium displays high intrinsic resistance to a wide range of antibiotics. This is mainly caused by the low permeability of its outer membrane, which on the outer surface is densely packed with lipopolysaccharide molecules. The outer membrane also contains multiple embedded proteins, which provide the only effective passage for the bacterial cell to exchange matter with the environment. About 70 different outer membrane proteins transport a variety of compounds across the outer membrane of $P$. aeruginosa. Some of them have been studied in some detail, but the exact substrate specificity and the mechanism of transport of many of them remains unknown.

In this dissertation I present a detailed structural and functional characterization of two outer membrane proteins from $P$. aeruginosa-OprG and OprH. OprG is a member of the OmpW family of proteins whose function as an antibiotic-sensitive porin has been controversial and not well defined. Circumstantial evidence led to the proposal that OprG might transport hydrophobic compounds by using a lateral gate in the barrel wall thought to be lined by three conserved prolines. To test this hypothesis and to find the physiological substrates of OprG, the purified protein was reconstituted into liposomes and was found to facilitate the transport of small amino acids such as glycine, alanine, 
valine, and serine, which was confirmed by Pseudomonas growth assays. The structures of the wild-type protein and a critical proline mutant were determined by nuclear magnetic resonance spectroscopy in dihexanoyl-phosphatidylcholine micellar solutions. Both proteins formed eight-stranded $\beta$-barrels with flexible extracellular loops. The interfacial prolines did not form a lateral gate in these structures, but loop 3 exhibited restricted motions in the inactive P92A mutant, but not in wild-type OprG.

The function of OprH is to provide increased stability to the outer membranes of $P$. aeruginosa by directly interacting with LPS molecules. The NMR solution structure of OprH reveals an eight-stranded $\beta$-barrel with four extracellular loops of unequal size. Based on NMR chemical shift perturbations observed upon the addition of LPS to OprH in lipid micelles, I concluded that the interaction is predominantly electrostatic and localized to charged regions near upper rim of the barrel. By applying site-directed mutagenesis and ELISA I was able to identify OprH residues that are essential for the interaction with LPS. The results of this study provide a more definitive molecular model for the binding of LPS to OprH and offer new insight into protein-lipid interactions that likely contribute to the antibiotic resistance during $P$. aeruginosa infections. 


\section{Dedication}

To my mother, Alicja Samek, for her encouragement and support. 


\section{Abbreviations}

AMP, antimicrobial peptide;

BLAST, basic local alignment search tool;

BM2, basal medium-2;

$\mathrm{BR}$, bronchiectasis;

CBQCA, 3-(4-carboxybenzoyl)quinolone-2-carboxaldehyde;

$\mathrm{CF}$, cystic fibrosis;

Dab, $\alpha, \gamma$-diaminobutyric acid;

DDM, lauryl- $\beta-D$-maltoside;

DHPC, 1,2-dihexanoyl-sn-glycero-3-phosphocholine;

DHPS, 1,2-dihexanoyl-sn-glycero-3-phospho-L-serine;

DMPC, 1,2-dimyristoyl-sn-glycero-3-phosphocholine;

DMPS, 1,2-dimyristoyl-sn-glycero-3-phospho-L-serine;

DPC, dodecylphosphocholine;

DSS, 2,2-dimethyl-2-silapentane-5-sulfonate;

EDTA, ethylenediaminetetraacetic acid;

ELISA, enzyme-linked immunosorbent assay;

EtNP, ethanolamine phosphate;

FhuA, ferric hydroxamate uptake $A$;

GalN, N-acetyl galactosamine; 
Glc, glucose;

Hep, L-glycero-D-manno-heptose;

HEPES, 4-(2-hydroxyethyl)-1-piperazineethanesulfonic acid;

HSQC, heteronuclear single quantum coherence spectroscopy;

Kdo, D-manno-oct-2-ulosonic acid;

LB, lysogeny broth;

LBP, LPS-binding protein;

LMPG, 1-myristoyl-2-hydroxy-sn-glycero-3-phospho-(1'-rac-glycerol);

LPS, lipopolysaccharide;

MALDI-TOF, matrix assisted laser desorption ionization time-of-flight;

MD-2, myeloid differentiation protein-2;

MSP, membrane scaffold protein;

NMR, nuclear magnetic resonance;

NOE, nuclear Overhauser effect;

NOESY, nuclear Overhauser effect spectroscopy;

O.D., optical density;

OM, outer membrane;

OMP, outer membrane protein;

OprG, outer membrane protein G;

OprH, outer membrane protein $\mathrm{H}$;

$\mathrm{PCR}$, polymerase chain reaction; 
PG, peptidoglycan;

PmB, polymyxin B;

POPC, 1-palmitoyl-2-oleoyl-sn-glycero-3-phosphocholine;

POPG, 1-palmitoyl-2-oleoyl-sn-glycero-3-phosphoglycerol;

POPS, 1-palmitoyl-2-oleoyl-sn-glycero-3-phosphatidylserine;

r.m.s.d., root mean square deviation;

Rha, rhamnose;

SDS, sodium dodecyl sulfate;

TEV, tobacco etch virus;

TLR, toll-like receptor;

TOCSY, total correlation spectroscopy;

TRACT, TROSY for rotational correlation times;

TROSY, transverse relaxation-optimized spectroscopy;

WT, wild-type. 


\section{Table of Contents:}

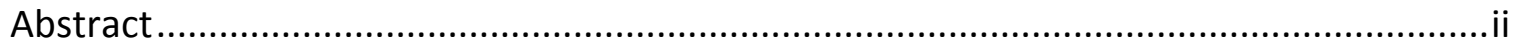

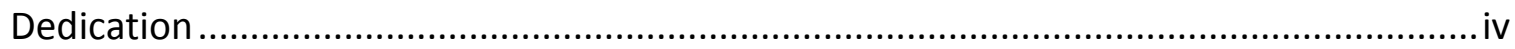

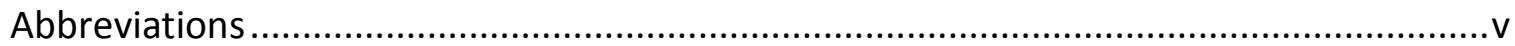

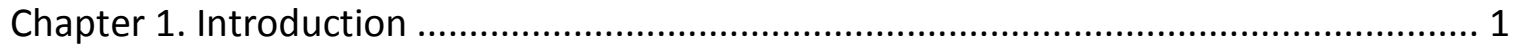

$1.1 \mathrm{~A}$ brief introduction to Pseudomonas aeruginosa ............................................... 1

1.2 Lipopolysaccharide (LPS) of P. aeruginosa ......................................................... 2

1.2.1 Structure and chemical composition of $P$. aeruginosa .................................. 2

1.2.2 Interaction of $P$. aeruginosa LPS with antimicrobial peptides (AMPs) ............... 7

1.2.3 Interaction of $P$. aeruginosa LPS with the innate immune system .................. 10

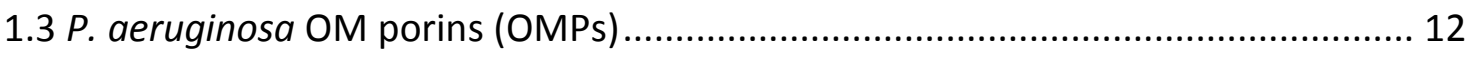

1.4 Outer membrane protein G (OprG) from P. aeruginosa ................................... 15

1.5 Outer membrane protein $\mathrm{H}(\mathrm{OprH})$ and PhoP-PhoQ two-component system ...... 17

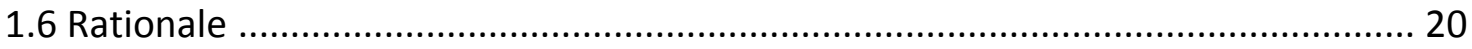

Chapter 2. OprG harnesses the dynamics of its extracellular loops to transport small

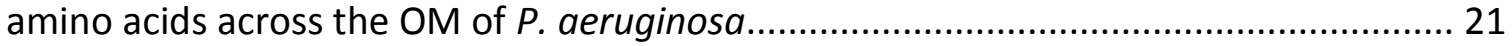

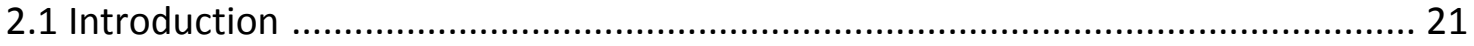

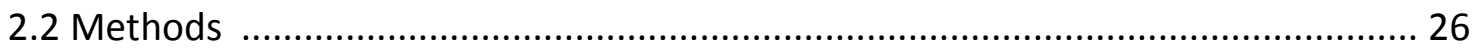

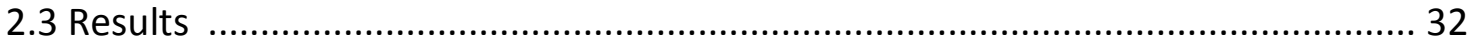

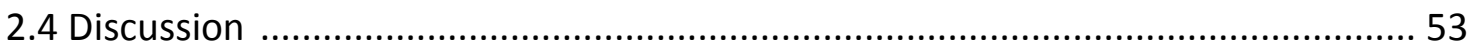

Chapter 3 Molecular Interactions of Lipopolysaccharide with an Outer Membrane Protein from Pseudomonas aeruginosa Probed by Solution NMR ......................................... 59

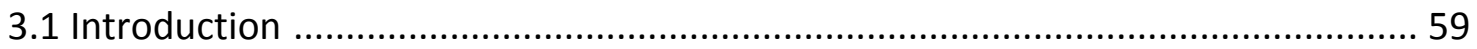

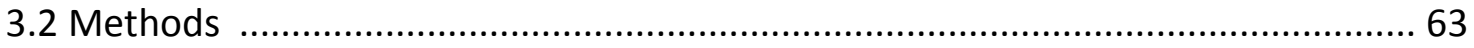

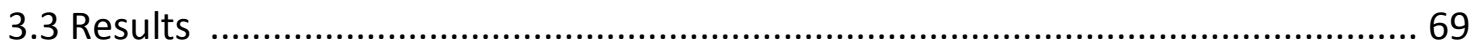

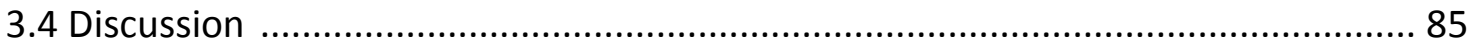


Chapter 4. Optimizing nanodiscs and bicelles for solution NMR studies of two $\beta$-barrel

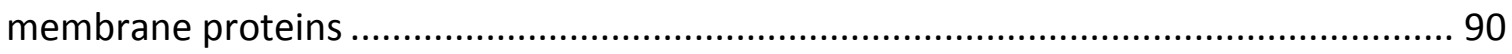

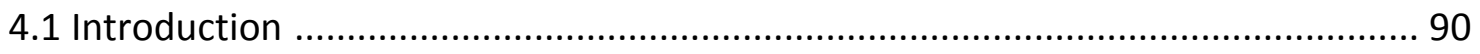

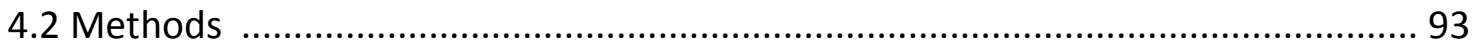

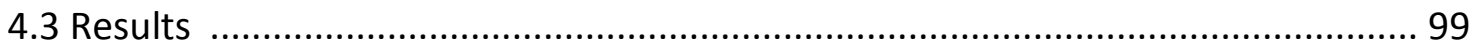

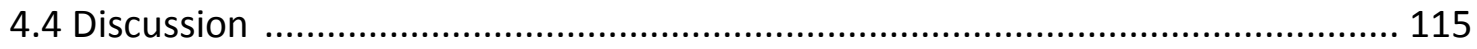

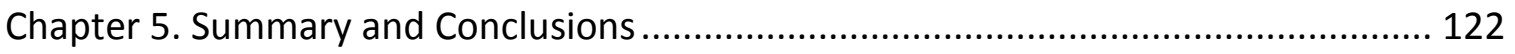

Appendix 1. Investigation of the OprG oligomerization state .................................. 126

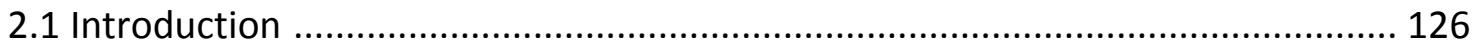

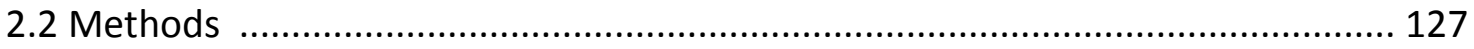

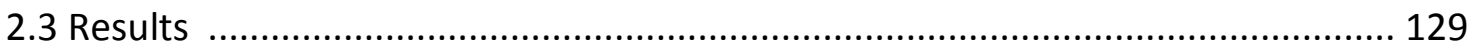

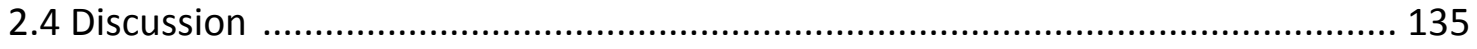

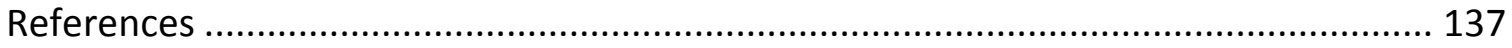




\section{Chapter 1. Introduction}

\subsection{A brief introduction to Pseudomonas aeruginosa}

Pseudomonas aeruginosa is a Gram-negative ubiquitous microorganism commonly found in aquatic and soil habitats, animals and plants [1]. Encoding 5570 genes on 6.3 mega base pairs of DNA, the genome of $P$. aeruginosa strain PA01 is one of the largest known bacterial genomes. The nature of the genes that have been described is consistent with the versatility of this organism, including four motility systems, a large number of systems for metabolism of carbon sources, one of the highest percentages of regulators (nearly 1 gene in 9) of any genome to date, and many different transport systems [2, 3]. P. aeruginosa is also an opportunistic human pathogen, particularly affecting immunocompromised patients or those admitted to the intensive care unit [4, 5]. This pathogen is an especially dangerous driver of chronic respiratory infections to patients suffering from cystic fibrosis (CF) or other chronic underlying diseases such as bronchiectasis and chronic obstructive pulmonary disease [6]. The list of infections caused by $P$. aeruginosa also includes community acquired pneumonia, bacteriemia, urinary tract infections, folliculitis, keratitis, endophthalmitis, otitis, enterocolitis, osteomyelitis and meningitis $[1,4]$. Compared with other pathogens, $P$. aeruginosa infections are very 
difficult to treat as this microorganism displays high intrinsic resistance to a wide range of antibiotics, including fluoroquinolones, aminoglycosides, and $\beta$-lactams. This is mainly caused by the low permeability of its outer membrane (OM) which is estimated to be 12 to 100 times less permeable than that of $E$. coli $[7,8]$. The low OM permeability and the slow rate of penetration of antibiotic molecules enable secondary adaptive resistance mechanisms to function more efficiently, including increased efflux and enzymatic modifications of antibiotics [9]. Due to the increasing prevalence of transferable resistance determinants, particularly those encoding class $\beta$-carbapenemases or extended-spectrum $\beta$-lactamases [10-12], $P$. aeruginosa is often resistant to multiple antibiotics and consequently has joined the ranks of 'superbugs' [9].

\subsection{Lipopolysaccharide (LPS) of P. aeruginosa}

1.2.1 Structure and chemical composition of $P$. aeruginosa LPS. Cells of gramnegative bacteria such as $P$. aeruginosa are surrounded by two concentric lipid bilayer membranes. Both membranes contain proteins that allow the passage of matter and information, but their compositions and functions are very different (Fig. 1). The inner (cytoplasmic) membrane is exclusively composed of phospholipids, mainly phosphatidylethanolamine (70-80\%), cardiolipin and phosphatidylglycerol, distributed equally among the inner and outer leaflet. In contrast, the OM is highly asymmetric; the inner leaflet shows the same lipid composition as the cytoplasmic membrane and the 
outer leaflet consists almost exclusively of LPS molecules [13]. LPS is made of a hydrophobic lipid $A$, which is responsible for the cytotoxic properties of the molecule, a hydrophilic core polysaccharide chain, and a hydrophilic O-antigenic polysaccharide side chain (Fig. 2). The acyl chains of lipid A are tightly apposed to the acyl chains of the phospholipid molecules from the inner leaflet of the OM, thus contributing to the structural integrity of the OM [14].

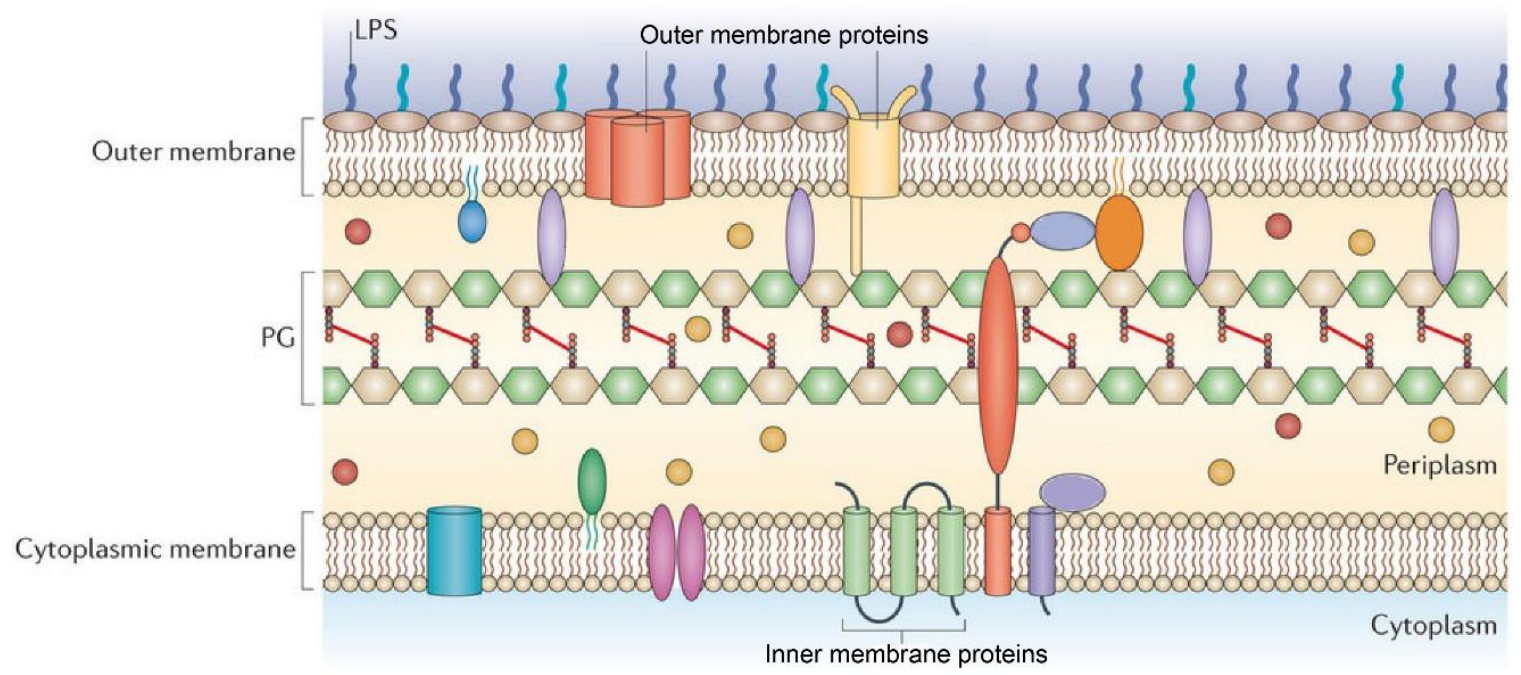

Figure 1. The cell envelope of Gram-negative bacteria consists of two membranes, the outer membrane and the cytoplasmic membrane. The cytoplasmic membrane is composed of a phospholipid bilayer, whereas the outer membrane comprises an interior leaflet of phospholipids and an exterior leaflet of LPS. In between the two membranes is the periplasmic space, which contains the peptidoglycan (PG) layer and periplasmic proteins. Envelope proteins can be soluble (periplasmic; orange and red spheres), transmembrane proteins (pink ovals and cyan cylinder) or anchored into the leaflet of either membrane via covalently attached lipid appendages (lipoproteins; green and blue ovals) [15]. 
a

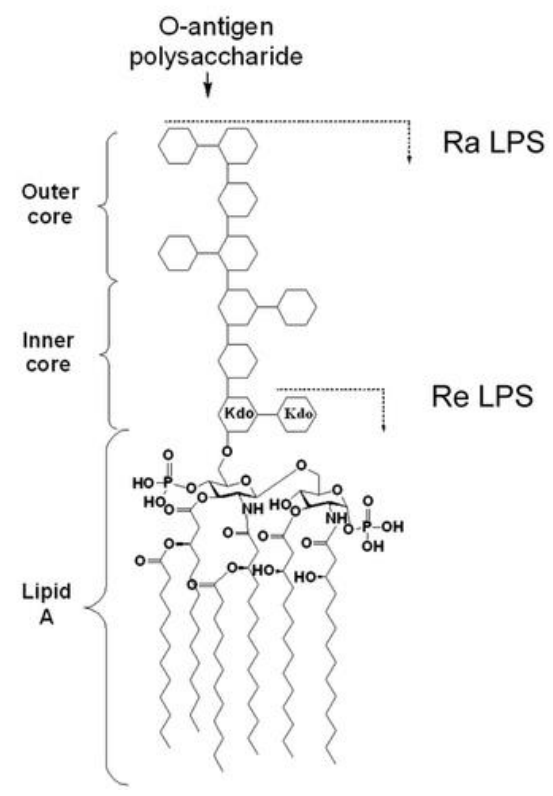

b

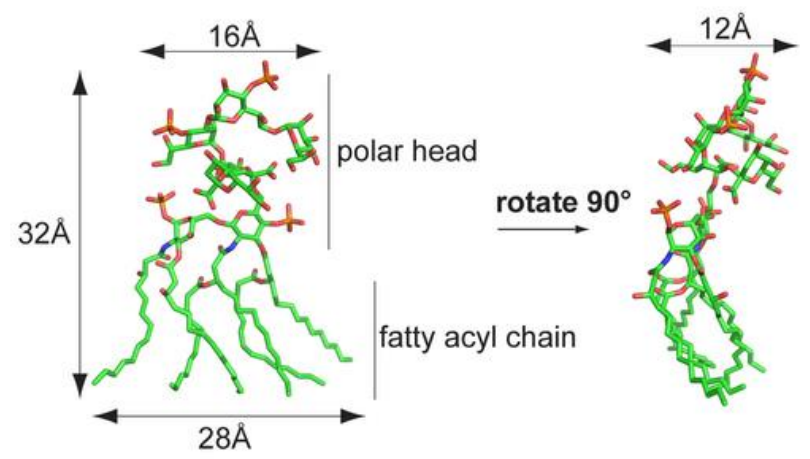

Figure 2. Chemical structure of E. coli LPS. A) LPS is composed of three modules: lipid A, a core oligosaccharide and a highly variable $\mathrm{O}$-antigen constituted of repeating oligosaccharide units. The core is covalently linked to lipid $A$ and can be further divided into inner and outer core. The inner core contains at least one residue of 3-deoxy-Dmanno-oct-2 ulosonic acid (Kdo) linking to lipid A. B) Ra LPS is about $32 \AA$ in height and $28 \AA \times 12 \AA$ in the other two dimensions. The size of Ra LPS is based on the crystal structure of the TLR4/MD-2/Ra LPS complex (PDB ID 3FXI) [16].
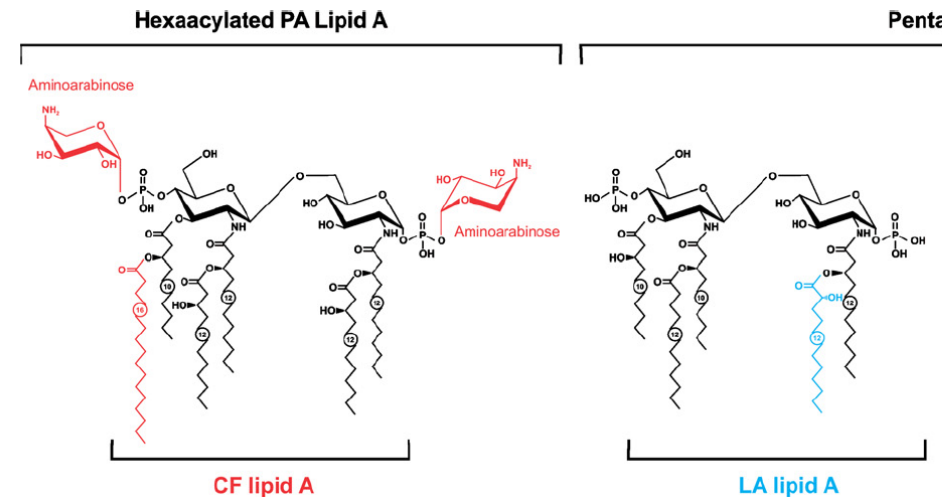

Pentaacylated PA Lipid A

Figure 3. Structures of variant $P$. aeruginosa lipid $A$ found to be predominantly expressed in isolates from CF patients (CF lipid A), bronchiectasis patients (BR lipid A) or from a laboratory-adapted (LA lipid A) strain, PAK [17]. 
LPS produced by $P$. aeruginosa is a main factor in virulence as well as innate and acquired host responses to infection. Its chemical structure is typical for gram-negative bacterial LPS, with a basic lipid A structure containing an $\mathrm{N}$ - and $\mathrm{O}$-acylated diglucosamine bisphosphate backbone with chemical variation in the number of primary acyl groups and the types of fatty acids substituting the primary and secondary acyl groups (Fig. 3). 75\% of the LPS molecules synthesized by laboratory adapted strains of $P$. aeruginosa are penta-acylated and $25 \%$ are made as a hexa-acylated LPS [17]. Growth conditions, especially concentration of magnesium ions, may influence the acylation pattern of $P$. aeruginosa lipid $\mathrm{A}[18,19]$. The hexa and hepta-acylated lipid A moieties may also contain cationic 4-amino-4-deoxy-L-arabinose sugars (Fig. 3). Bound to the lipid A is a relatively conserved inner core structure which contains two D-manno-oct-2-ulosonic acid residues (Kdol and Kdoll) and two L-glycero-D-manno-heptose residues (Hepl and Hepll) (Fig. 4) $[20,21]$. 7-O-carbamyl group is bound to the second heptose residue, Hepll [22], and is also found in the LPS of other types of pseudomonads [23]. The two heptose residues are often phosphorylated at positions 2 and 4 of Hepl and position 6 of Hepll [24-26]. Phosphate substituents are mainly mono- and di-phosphates, however, tri-phosphates are also commonly found, which is unique to $P$. aeruginosa. Phosphorylation of the $P$. aeruginosa LPS inner core is necessary for the bacterial survival; a mutation in the waaP gene that phosphorylates position 4 of the Hepl groups is lethal for the organism [27]. 
The outer core of the $P$. aeruginosa LPS is termed O-antigen, as it confers antigenic properties of this molecule. O-antigens are built of repeating units of oligosaccharides, the chemical structures of which are strain-specific. The outer cores of the $P$. aeruginosa LPS are usually synthesized as two different isoforms or glycoforms by individual strains $[20,24,26]$. Both isoforms contain an $\mathrm{N}$-alanylated galactosamine residue, three Dglucose residues and one L-rhamnose residue, the specific position of which depends on the isoform (Fig. 4). The monosaccharides are arranged in repeating units containing 3 or 4 individual monosaccharides [28]. P. aeruginosa strains that are unable to synthesize full length LPS are called "rough" strains, as the cell surface visualized under the electron microscope appears uneven. LPS-rough strains isolated from CF patients, have multiple mutations within the biosynthetic genes for the O-antigen [29] and do not have any Oantigen on glycoform 2 [17].

Purified LPS is highly heterogeneous and tends to form aggregates of varying sizes of 1-4 million Da or greater. Treatment with SDS and high temperature results in lowering the molecular weight to 50 to $100 \mathrm{kDa}$ [30]. Obtaining the purest form of LPS (10-20 kDa) requires the presence of strong surface active agents in the absence of divalent cations. The smallest forms of LPS in solution are 1000 kDa micelles formed in the presence of divalent cation sequestering agents such as EDTA. Upon treatment with divalent cations such as $\mathrm{Ca}^{2+}$ and $\mathrm{Mg}^{2+}$, a bilayer structure is formed that passes through a $0.2 \mu \mathrm{m}$ membrane, but not a $0.025 \mu \mathrm{m}$ membrane [31]. LPS can also form vesicles with up to 0.1 
$\mu \mathrm{m}$ in diameter in solution when divalent cations are present. The self-aggregation of LPS and the ability to bind to hydrophobic surfaces is believed to be a function of the lipid A component of the molecule [32].

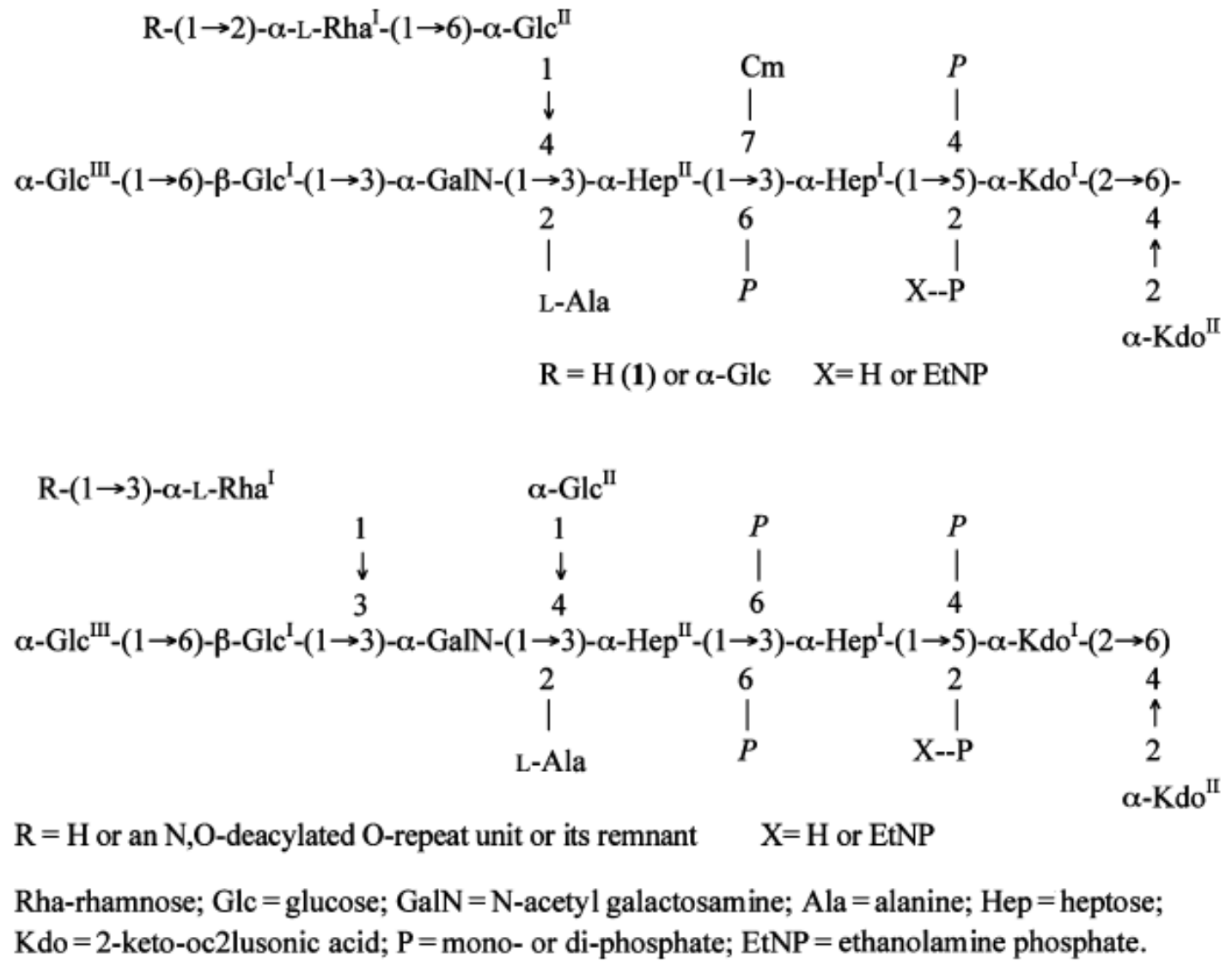

Figure 4. General structure of the two glycoforms of the $\boldsymbol{P}$. aeruginosa LPS core. The inner core comprises the two Kdo and two heptose (Hep) residues; the outer core the remainder of the molecule. Rha = rhamnose; Glc = glucose; GalN = N-acetyl galactosamine; $\mathrm{Ala}=$ alanine; $\mathrm{Hep}=$ heptose; $\mathrm{Kdo}=2$-keto-oc2lusonic acid; $\mathrm{P}=$ mono- or di-phosphate; EtNP = ethanolamine phosphate [17].

\subsubsection{Interaction of $\boldsymbol{P}$. aeruginosa LPS with antimicrobial peptides (AMPs). The}

binding of LPS to proteins and peptides is important in many biological responses. In 
recent years, AMPs have gained considerable attention as potential therapeutic agents for the treatment of bacterial infections, including sepsis [33]. AMPs are an important component of the innate immune system and the first line of defense against invading bacterial pathogens [34-37]. Nearly all known AMPs have a net positive charge due to an abundance of lysine and arginine residues and a high fraction (20-50\%) of hydrophobic residues, especially tryptophan [33]. AMPs are able to reach lytic concentrations near anionic bacterial surfaces by electrostatic attraction while adsorption and membrane rupture are further facilitated by the amphiphilicity of those peptides. Different mechanisms have been noted for AMP-induced bacterial membrane rupture, including formation of barrel-stave or toroidal pores and disruption of packing of LPS by a detergent-like mechanism. An important aspect of AMPs functionality is their selectivity, which is necessary to ensure that pathogen cells are efficiently destroyed while human cells are left intact. This selectivity is based on the different composition of human and bacterial membranes. The outer surfaces of bacterial membranes mainly contain anionic LPS while human cell membranes are rich in cholesterol and zwitterionic lipids, such as phosphatidylcholine and sphingomyelin, thus generally bearing much lower charge [33, 38].

Polymyxin $\mathrm{B}(\mathrm{PmB})$ is a highly cationic decapeptide antibiotic, which interacts with LPS as thoroughly characterized in several publications [39-42]. PmB contains a macrocylic ring including residues 4 to 10, six diaminobutyric acid residues and an acyl 
chain connected to the $\mathrm{N}$ terminus (Fig. 5). Due to severe toxic side effects, PmB is used only in treatments against highly antibiotic-resistant pathogens such as $P$. aeruginosa [3941, 43-49]. Mares et al [41] modeled the complex of LPS with PmB in the presence of dodecylphosphocholine micelles using molecular dynamics calculations with restraints derived from chemical shift mapping data and intermolecular nuclear Overhauser effects. In this complex the macrocyclic ring of PmB is centered on the phosphate group of $\mathrm{N}$ acylated diglucosamine B of LPS and additional contacts from polar side chains are formed to $\mathrm{N}$-acylated diglucosamine $\mathrm{A}$ and Kdol, while the hydrophobic side chains of Phe and Leu penetrate the acyl-chain region [41]. The importance of electrostatic interactions between phosphate groups of LPS and amino groups is also highlighted by other available structures of LPS-bound peptides and proteins. The structure of a LPS-bound fragment of the LALF protein [47] reveals a hairpin-type fold with several hydrophobic and cationic residues participating in binding to LPS. Cationic amino acids have also been postulated to critically contribute to the binding of LPS to the lytic bee venom peptide melittin [45] and proteins such as MD-2 [50] or FhuA [51, 52].

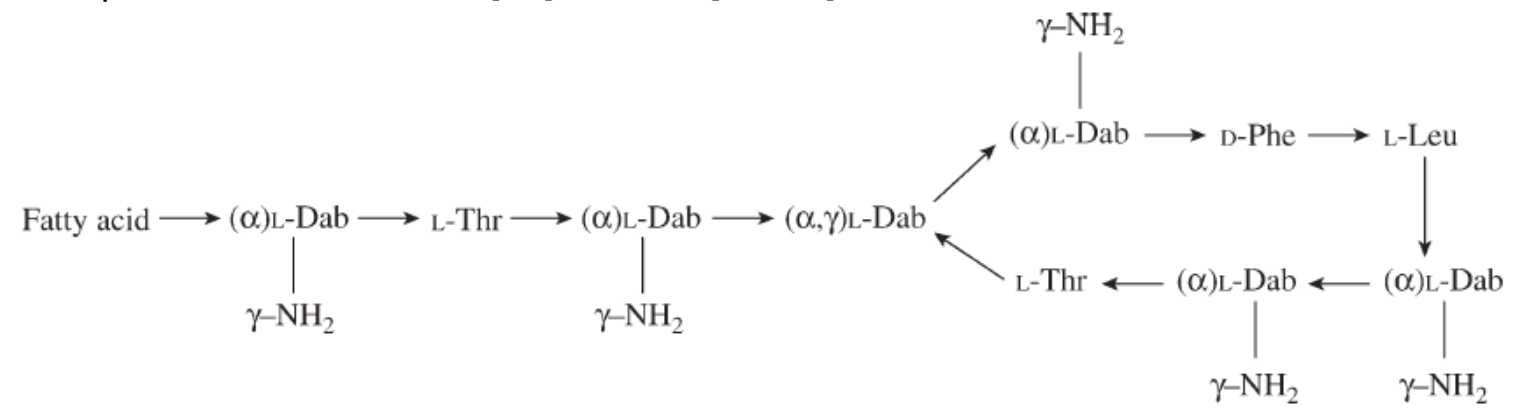

Figure 5. Structure of polymyxin B. Fatty acid: 6-methyloctanoic acid for polymyxin B1, 6-methylheptanoic acid for B2, octanoic acid for B3 and heptanoic acid for B4. Thr, threonine; Leu, leucine; Dab, $\alpha, \gamma$-diaminobutyric acid; Phe, phenylalanine; where $\alpha$ and $\gamma$ indicate the respective amino group involved in the peptide linkage [53]. 
1.2.3 Interaction of $\boldsymbol{P}$. aeruginosa LPS with the innate immune system. Toll-like receptors (TLR) are an integral part of the mammalian immune system responsible for its activation during bacterial infections. They are usually present in cell membranes of macrophages and dendritic cells and are activated by various molecules derived from microbes [54]. TLR4 is one of the better understood TLRs, and it is responsible for the recognition of the lipid A part of LPS. It is essential for the effective response of the host immune system and for pathological effects resulting from LPS-induced sepsis [55, 56]. Recognition of lipid A by TLR4 is a complex process which involves several extracellular proteins as well as intracellular signal-transducers. The first step of activation is binding of LPS by LPS-binding protein (LBP), a 60-kDa glycosylated polypeptide, which is constitutively synthesized by the liver [57]. Subsequently, LPS is transported by LBP to membrane-bound CD14, which constitutes a part of the LPS-binding complex. CD14bound LPS is able to interact with TLR4 in the presence of the co-factor MD2. It is not completely understood if this occurs on the cell surface or in the endosomes after bacteria or LPS are engulfed by phagocytosis or endocytosis [58]. Interaction of LPS with TLR4 recruits adaptor proteins containing Toll/IL-1 receptor (TIR) domains such as TIRAP/Mal and TRAM, which are essential for the signal transduction by the TLR4-MD2 LPS complex [55]. MyD88 and TRIF are other proteins which are essential for initializing the cellular response and activating various transcription factors, mainly NF-kB, in response to LPS. In the nucleus NF-kB promotes production of inflammatory cytokines, including interleukin 
(IL)-1, IL-6, IL-8 and tumor necrosis factor. The final host response to the presence of bacterial LPS is determined by the balance of production of these factors (Fig. 6) [17].

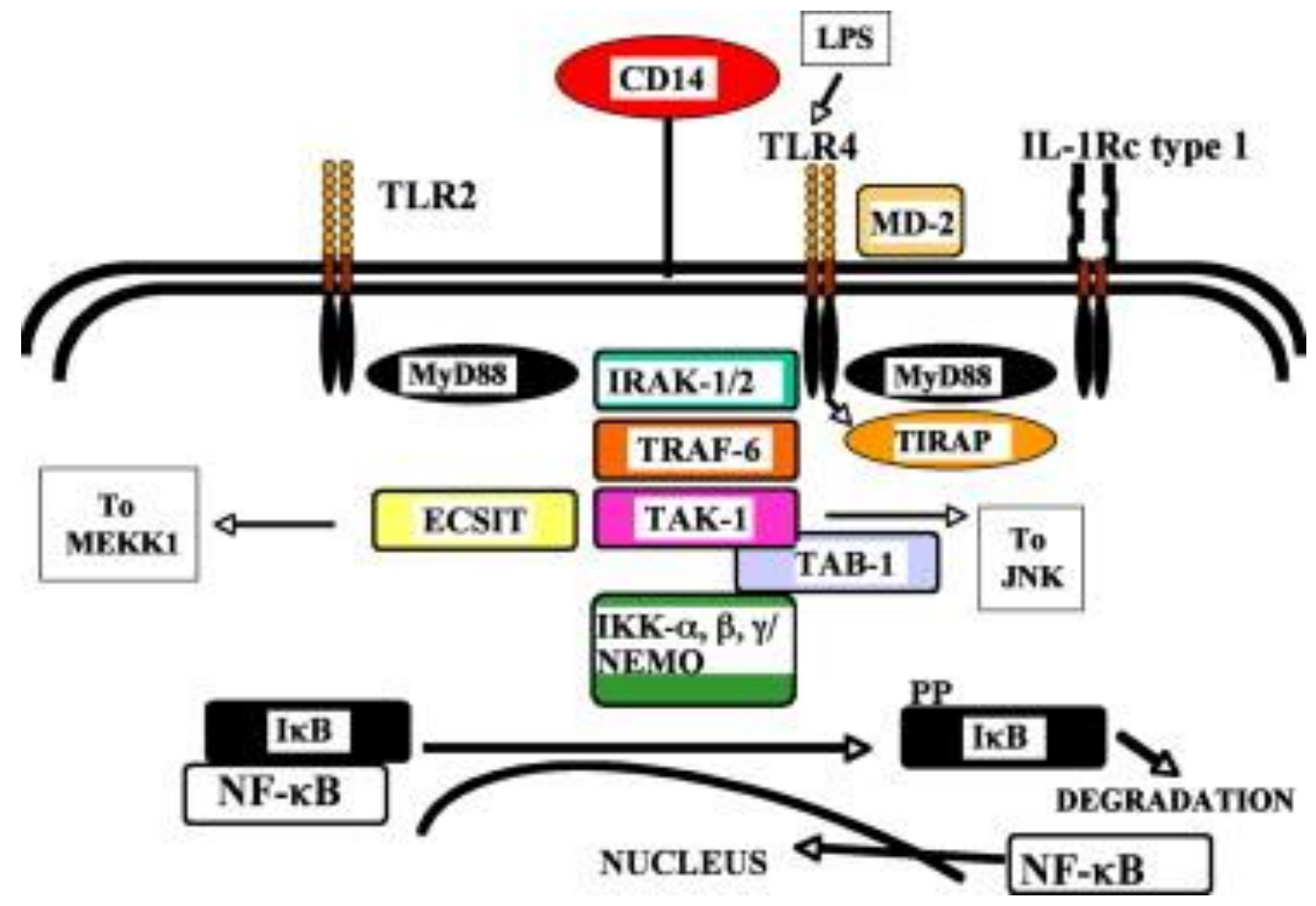

Figure 6. Activation of innate immune signaling pathways by binding of LPS to TLR4-MD2, facilitated by CD14. TLR4, along with other TLRs such as TLR2, and cytokine receptors such as the interleukin-1 receptor type 1 (IL-1Rc type 1) utilize a variety of adaptor molecules to transmit the information of ligand binding to the nucleus in order to induce cytokine and other responses. MyD88=myeloid differentiation factor 88; TIRAP=TIR domain-containing adaptor protein; TRAF-6=tumor necrosis factor receptor-associated factor 6; TAK-1=transforming growth factor $b$-activating kinase 1 ; TAB1 TAB2, and TAB3=TAK1-binding protein 1, 2 or 3; IKK- $\alpha,-b$, and $-\gamma$. IKK- $\gamma=$ inducible kinase, IKK- $\gamma$ is also called NEMO (nuclear factor $\kappa \mathrm{B}(\mathrm{NF}-\kappa \mathrm{B})$ essential modulator); I $\kappa \mathrm{B}=$ inhibitor of NF- $\kappa \mathrm{B}$; PPI $K \mathrm{~B}=$ phosphorylated I $\kappa \mathrm{B} ; \mathrm{JNK}=\mathrm{c}$-Jun $N$-terminal kinase [17].

The LPS synthesized by $P$. aeruginosa often produces a weaker response from the innate immune system compared to LPS of other enterobacteria [59]. This is due to the high degree of variability in the structure of $P$. aeruginosa lipid $A$, which depends on the 
strain and growth conditions. The TLR4-mediated response to the presence of LPS is largely dependent on the degree of acylation of lipid A; the presence of fully hexaacylated lipid A generates a stronger immune response while production of lipid A with low levels of acylation weakens the production of the inflammatory cytokines and the host immune response [18]. Strains isolated from chronically infected lungs of CF patients produce more of the hexa-acylated LPS, which results in a stronger inflammatory response when tested on cells in vitro compared to penta-acylated LPS produced by nonCF strains [19].

\subsection{P. aeruginosa OM porins (OMPs)}

Proteins are important components of the OM of Gram-negative bacteria and they are estimated to constitute as much as $50 \%$ of the OM mass. OMPs can be present either in the form of integral membrane proteins or as lipoproteins anchored to the membrane via N-terminally attached lipids [60]. Functions on OMPs are very diverse, including maintenance of cell structure, passive and active transport, adhesion to other cells, binding a variety of substances, and antimicrobial resistance in microorganisms [61].

Whereas the number of distinct chain folds of water-soluble proteins seems to range around 1000 [62], the respective number of possible folds of integral membrane proteins is much smaller, as only regular secondary structure elements can occur within the lipid bilayer. As the dielectric constant within a lipid bilayer is very low compared with 
that of the aqueous environment, membrane proteins expose a hydrophobic surface to the lipid bilayer core. This implies that a maximum of intramolecular hydrogen bonds of the protein domain located in the lipid bilayer is formed. All donor and acceptor groups could be saturated either intrasegmentally, as in the case of $\alpha$-helices, or intersegmentally by the formation of hydrogen bonds between adjacent $\beta$-strands [63]. In agreement with this prediction all known structures of integral membrane proteins are either built of $\alpha$ helices that run perpendicular to the membrane plane or antiparallel $\beta$-pleated sheets forming a closed barrel. The proteins located in cytoplasmic membranes are mostly built of $\alpha$-helical bundles, while $\beta$-barrels can be found in OM of bacteria, mitochondria and chloroplasts. This difference in localization is believed to be due to the different biogenesis of OM proteins; OMPs first have to be transported across the cytoplasmic membrane as unfolded polypeptides, which would not be possible if they were already folded and therefore too hydrophobic $[13,64]$. All known bacterial $\beta$-barrel proteins have similar folds with even numbers of $\beta$-strands and their $N$ and $C$ termini located at the periplasmic barrel end. Another parameter that describes the architecture of $\beta$-barrel proteins is the shear number [65]. This number measures the stagger of the strands in the $\beta$-sheet. Optimal shear numbers for the least stressed $\beta$-barrels, $S_{0}$, have been predicted as a function of the number of strands, $n$, to be $S_{o}=n+4$. The number of strands and shear number determine the major geometrical features of $\beta$-barrels, such as the mean slope of the strands to the axis of the barrel (referred to as tilt angle) and the mean radius of the barrel [64]. All $\beta$-strands of bacterial OMPs are antiparallel and connected to their 
neighboring strands through long external loops or short periplasmic turns of a few residues [66]. The extracellular connections can be very mobile, with exceptionally high sequence variability. Another common structural feature of $\beta$-barrel proteins is the presence of two girdles of aromatic side chains, which have intermediate polarity and anchor the protein in the nonpolar-polar interfaces of the membrane lipid bilayer [67].

The majority of known bacterial OMPs are involved in the transport of various substances across the OM. This transport is generally passive, as the lack of ATP or any equivalent energy carrier in the periplasm renders the OMPs unable to transport solutes against a concentration gradient. The channels present in the OM can be differentiated by the nature of their interaction with the substrates. The non-specific channels do not bind their substrates with measurable affinities $[68,69]$ and work most efficiently when the external substrate concentrations are high. The second class of channels consists of substrate specific channels, which have binding sites for the molecules they transport. Under low substrate concentration conditions they are more efficient than non-specific channels and therefore are especially common in microbes from nutrient-poor environments, such as pseudomonads. In fact, pseudomonads do not have any unspecific OM channels and the transport of nutrients into their cells relies only on about 40 different substrate-specific porins $[70,71]$. Despite the OM permeability of approximately $8 \%$ that of E. coli, P. aeruginosa has a quite large OM exclusion limit, permitting passage of compounds of around 3000 molecular weight compared to an exclusion limit of around 500 molecular weight for E. coli [72]. 


\subsection{Outer membrane protein G (OprG) from $P$. aeruginosa}

OprG is a small OM protein from $P$. aeruginosa and a member of the OmpW family of OMPs $[73,74]$. Despite their prevalence in Gram-negative bacteria, proteins from this family remain poorly characterized. Various reports showed that OmpW family members are highly immunogenic [75-77] and that E. coli OmpW is a receptor for colicin S4, which constitutes a part of a bacteriocin defense system $[75,78]$. Other possible functions of OmpW family members include osmoregulation [79], adaptive responses to

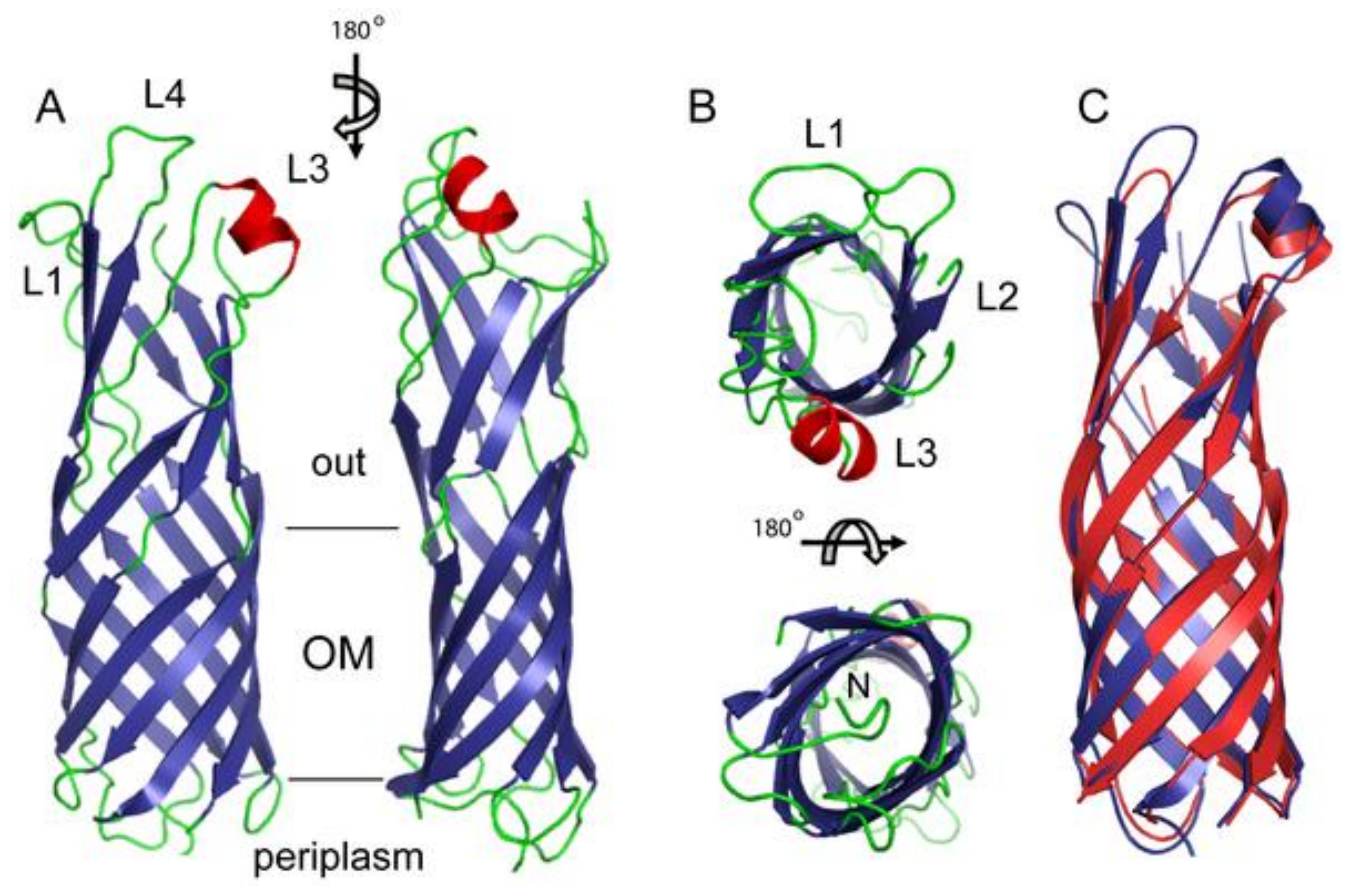

Figure 7. Structural overview of OprG. Views from the side (A) and from the extracellular side (B; top panel) and periplasmic side (b; bottom panel). $\beta$-strands are colored blue, $\alpha$-helices red and loops green. Selected extracellular loops are indicated. The approximate positions of the outer membrane interface regions are indicated by horizontal lines. (C) Structural comparison between OprG (blue) and E. coli OmpW (red). Loops have been smoothed for clarity [74]. 
environmental conditions [80], and uptake of naphthalene and other polycyclic aromatic compounds [81].

One of the first reports of OprG comes from Yates et al. [82] who showed that this protein was highly expressed in iron-rich conditions and therefore suggested that OprG might be a low affinity iron transporter. Several reports implied a role of OprG in $P$. aeruginosa antibiotic resistance to kanamycin, tetracycline and norfloxacin [83, 84], however none of this reports were confirmed by analysis of oprG knockout strains [73]. It is clear that the levels of OprG change substantially under a variety of different conditions suggesting a complex regulation. Interestingly, OprG was also proposed to be functionally important at early stages of pathogen-cell interaction and facilitate $P$. aeruginosa infections. McPhee et al. [73] found that oprG-deficient strains of $P$. aeruginosa are less cytotoxic to human bronchial epithelial (HBE) cells than wild-type strains. An oprGdeficient $P$. aeruginosa strain shows $1 \%$ cell lysis after 4 hour incubation while a strain expressing wild-type OprG is capable of causing $42 \%$ HBE cell lysis.

The crystal structure of $P$. aeruginosa OprG reveals an 8-stranded $\beta$-barrel with four long extracellular loops, three short periplasmic turns and an interior of the pore that is lined with several hydrophobic residues (Fig. 8) [74]. This architecture resembles closely the E. coli homolog OmpW [85]. The OprG crystal structure features a lateral opening in the barrel wall formed by three conserved prolines; Pro66, Pro91, and Pro92. As this lateral gate is localized near the extracellular hydrophilic-hydrophobic interface of the OM, it was suggested that it might be utilized for the transport of small hydrophobic 
molecules from the polar LPS-penetrating portion of OprG into the hydrocarbon layer of the OM in a fashion similar to a mechanism that has been proposed for the FadL-mediated transport of fatty acids in $E$. coli $[74,86,87]$. However, the transport of apolar compounds by OprG has so far only been speculated. Another indication of the possible function of OprG as transporter of hydrophobic substrates is the sequence similarity to OM proteins present in operons dedicated to the biodegradation of small, hydrophobic molecules such as naphthalene (NahQ and DoxH) and alkanes (AlkL) [88-90]. Neher et al. [81] demonstrated that OmpW from Pseudomonas fluorescens (70\% sequence similarity to OprG) is necessary for this organism growth on naphthalene, which was the first direct evidence of a function of a OmpW family member.

\subsection{Outer membrane protein $\mathrm{H}(\mathrm{OprH})$ and PhoP-PhoQ two-} component system

OprH is a $21-k D a, 200-$ residue, slightly basic protein that is integral to the OM of P. aeruginosa [91]. The solution NMR structure of OprH in 1,2-dihexanoyl-sn-glycero-3phosphocholine (DHPC) micelles reveals an eight-stranded $\beta$-barrel protein with four extracellular loops of unequal size (Fig. 7). Fast time-scale NMR dynamics measurements show that the extracellular loops are disordered and undergo fast motions [92]. When $P$. aeruginosa is cultured under low divalent cation concentration conditions, OprH is upregulated and overexpressed so that it becomes a major component of the OM. It is 
believed that OprH acts as a surrogate for $\mathrm{Mg}^{2+}$ and $\mathrm{Ca}^{2+}$ by cross-linking LPS, thereby tightening the OM during divalent cation deficiency [93]. The direct interaction of LPS with OprH was demonstrated using several in vitro and in vivo techniques. Based on NMR chemical shift perturbations observed upon the addition of LPS to OprH in lipid micelles, it can be concluded that the interaction is predominantly electrostatic and localized to charged regions near the upper rim of the barrel [92].
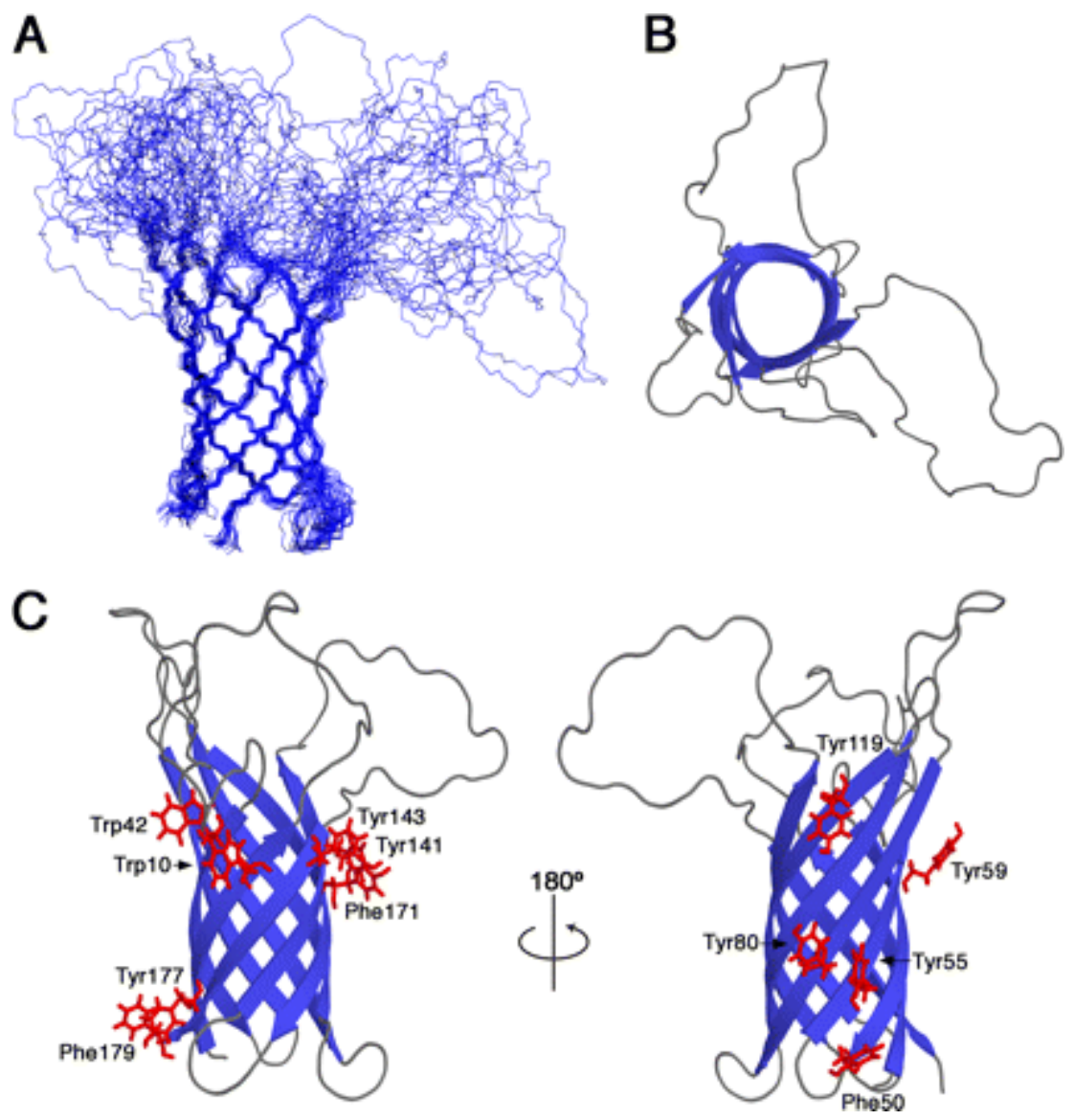

Figure 8. Solution structure of OprH in DHPC micelles. A, NMR ensemble of the 20 lowest energy structures calculated. B, top-down view of the lowest energy conformer of OprH from the ensemble of $\mathbf{2 0}$ lowest energy structures. C, two side views of the lowest energy conformer with the side chains of aromatic residues (red) located in the ordered $\beta$-barrel region are shown. The $\beta$-barrel and loop/turn regions are colored blue and gray, respectively, in $\mathbf{B}$ and $\mathbf{C}$ [92]. 
OprH is genetically linked to the PhoP-PhoQ two-component regulatory system that is up-regulated in response to $\mathrm{Mg}^{2+}$-limited growth conditions $[8,93]$. Twocomponent regulatory systems are a ubiquitous family of proteins that allow bacteria to modulate the expression of genes in response to various environmental cues. The pleiotropic nature of these systems makes them exceptionally efficient at mediating adaptive changes to environmental stress, and as a result many are involved in the virulence and antibiotic resistance pathways of pathogenic bacteria. Classic twocomponent regulatory systems comprise a membrane-associated sensor kinase and a DNA-binding response regulator. The sensor kinase responds to specific environmental stimuli by autophosphorylation at a conserved histidine residue at the expense of ATP. A transphosphorylation reaction follows, by which the kinase activates its cognate regulator protein; this phosphoprotein in turn activates or represses its target genes by binding to specific upstream sequences $[93,94]$. In $P$. aeruginosa, two separate two-component regulatory systems, PmrA-PmrB [95] and PhoP-PhoQ [96] are known to respond to the presence of limiting concentrations of $\mathrm{Mg}^{2+}$ and to separately regulate certain operons. The PhoPQ system autoregulates the oprH-phoP-phoQ operon [96] under $\mathrm{Mg}^{2+}$ limiting growth conditions and is also involved in virulence and in resistance to cationic antimicrobial peptides and polymyxin B [93]. According to study by Gellatly et al. [97], in bacteria that adhere to human bronchial epithelial cells, phoP and oprH were upregulated 116 and 5,000-fold, respectively, compared to bacterial cells that did not adhere. This was despite the fact that the cell culture medium contained millimolar 
concentrations of $\mathrm{Mg}^{2+}$ that would normally suppress transcription from the oprH-phoPphoQ operon [96].

\subsection{Rationale}

$P$. aeruginosa is a successful opportunistic pathogen that shows high multidrug resistance and has a strong evolutionary capability to obtain new mechanisms of resistance. Protective biofilms produced by $P$. aeruginosa further decrease the efficiency of known antibiotics [98]. Currently, the prognosis of $P$. aeruginosa infections is poor, with mortality rates reaching $50 \%[99,100]$. This highlights the importance of research concerning $P$. aeruginosa virulence and antibiotic resistance mechanisms.

The contribution of multiple $P$. aeruginosa porins to this organism infectivity and host-pathogen interactions is not well understood. In this work I discuss a structural and functional characterization of two OM proteins form this pathogen, OprG and OprH, and their role in $P$. aeruginosa lifestyle. We aim to elucidate the interaction of LPS with OprH in vitro and in vivo, and identify interacting residues that could potentially be targeted in studies aimed at advancing our understanding of pseudomonal antibiotic resistance. Since previous reports link OprG and antibiotic resistance [101] as well as ability to infect human cell lines [73], we propose to use biophysical and biochemical techniques to elucidate key attributes of OprG structure and function. We will use solution NMR spectroscopy, liposome-swelling, bacterial growth assays and other relevant techniques to investigate the barrel region of OprG for a possible transport mechanism. 


\section{Chapter 2: OprG harnesses the dynamics of its}

\section{extracellular loops to transport small amino acids}

\section{across the OM of Pseudomonas aeruginosa}

Iga Kucharska, Patrick Seelheim, Thomas Edrington, Binyong Liang and Lukas K. Tamm

Structure. 2015 Dec 1;23(12):2234-45.

\subsection{Introduction}

Pseudomonas aeruginosa is a rod-shaped Gram-negative bacterium commonly found in many natural and man-made environments. It can utilize various sources of carbon, which contributes to its ecological success. $P$. aeruginosa is also a serious opportunistic human pathogen and the most common cause of lung infections in cystic fibrosis patients [102, 103]. Moreover, $P$. aeruginosa is responsible for staggering numbers of nosocomial infections in hospital environments, including urinary tract, burn, and wound infections. Pseudomonal infections are difficult to treat because of the bacterium's ability to form biofilms that are highly resistant to many common 
antibiotics. This extraordinary antibiotic resistance is partially imparted by a very stable OM (OM) with a permeability to small molecules that is much lower than that of most other Gram-negative bacteria [104]. Complex lipopolysaccharides (LPS) in the OM and divalent cation-mediated LPS interactions contribute to the extraordinary stability of pseudomonal OMs towards adverse environmental conditions.

About 30 different OM proteins (OMPs) transport a variety of substrates across the OM of $P$. aeruginosa $[2,105]$. Unlike in $E$. coli and other Gram-negative bacteria, no general non-specific porins appear to exist in the OM of Pseudomonas. Rather, specific porins (named Opr in Pseudomonas) for the required substrates have evolved. Owing to the highly polar saccharide layer of LPS, the OM forms an efficient barrier not only to water-soluble substrates, but also to hydrophobic compounds. Because Pseudomonas can grow on a variety of different substrates, it has developed transport methods for polar, apolar, and charged molecules [106].

One of the smaller Pseudomonas porins is OprG, which forms an 8-stranded $\beta$ barrel in the OM $[73,74]$. OprG is a member of the widely distributed, but still poorly characterized OmpW family of OMPs. Possible functions of OmpW family members include osmoregulation [79], adaptive responses to environmental conditions [80], and uptake of naphthalene and other polycyclic aromatic compounds [81]. Even though the substrate specificity of OprG has not been previously characterized in much detail, an analysis of close homologs (Fig. 9) suggests possible functions. OprG from P. putida, 

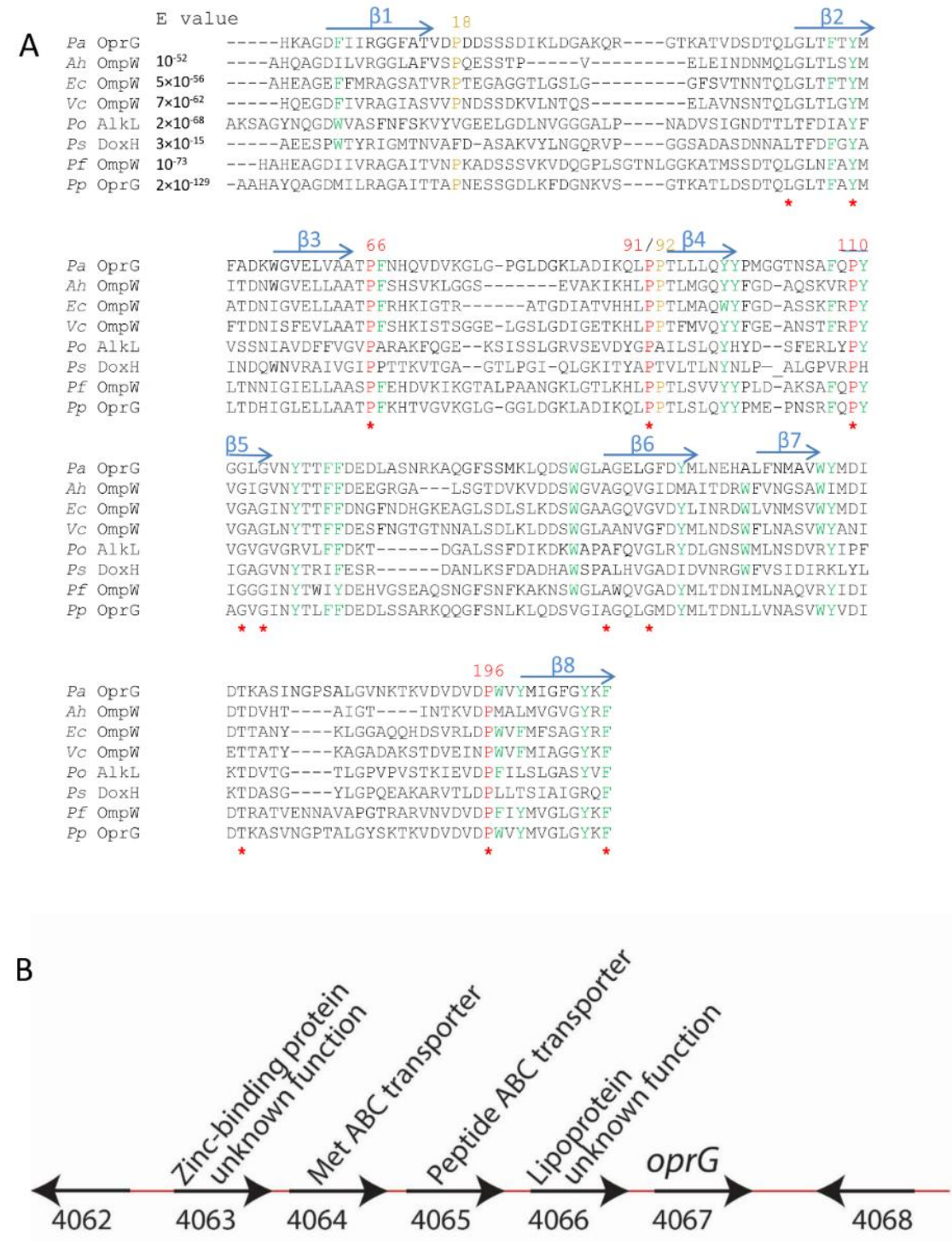

Figure 9. ClustalW alignment of OprG and closely related OmpW family members (A) and genomic context of oprG (B). A: The observed $\beta$-strands of OprG are shown above the alignment as blue arrows. All completely conserved residues are marked with a red star, fully conserved prolines are colored red, partially conserved prolines are colored orange, fully and partially conserved aromatic residues are colored green. The following OmpW family members have been used: $P a$, Pseudomonas aeruginosa OprG; $A h$, Aeromonas hydrophila OmpW; Ec, E. coli OmpW; Vc, Vibrio cholerae OmpW; Po, Pseudomonas oleovorans AlkL; Ps, Pseudomonas sp. (strain C18) DoxH; Pf, Pseudomonas fluorescens OmpW; Pp, Pseudomonas putida OprG. The expect values (E) were calculated using The Basic Local Alignment Search Tool (BLAST). B: Functional genes are indicated by the black arrows. Numbers below the graph indicate gene locus tags. Predicted function of the genes are indicated above the graph. 
which is $70 \%$ identical to $P$. aeruginosa OprG, is a protein of high emulsifying activity and may be involved in the transport of hydrocarbons [107]. OprG has $23 \%$ sequence homology with NahQ, a protein involved in dibenzothiophene and naphthalene catabolism in several species of Pseudomonas [88].

OprG has also been hypothesized to facilitate Pseudomonas infections. For example, oprG-deficient strains of $P$. aeruginosa are significantly less cytotoxic to human bronchial epithelial (HBE) cells than wild-type strains [73]. Wild-type P. aeruginosa causes $42 \%$ HBE cells to lyse after a 4 hour incubation, while an oprG-deficient strain shows only $1 \%$ cell lysis, suggesting that OprG plays a role at some early stage of pathogen-cell interaction.

The crystal structure of $P$. aeruginosa OprG reveals an 8-stranded $\beta$-barrel with four long extracellular loops, three short periplasmic turns, and an internal core lined with many apolar residues [74], i.e., its architecture closely resembles that of its $E$. coli homolog OmpW [85]. OprG features an interesting lateral opening in the barrel wall that is formed by three conserved prolines, Pro66, Pro91, and Pro92, which are all placed near the extracellular hydrophilic-hydrophobic interface of the OM (Fig. 10) Touw et al [74] hypothesized that this opening may serve as a lateral gate for the escape of small hydrophobic molecules from the polar LPS-penetrating portion of OprG into the hydrocarbon layer of the OM in a fashion similar to a mechanism that has been proposed 

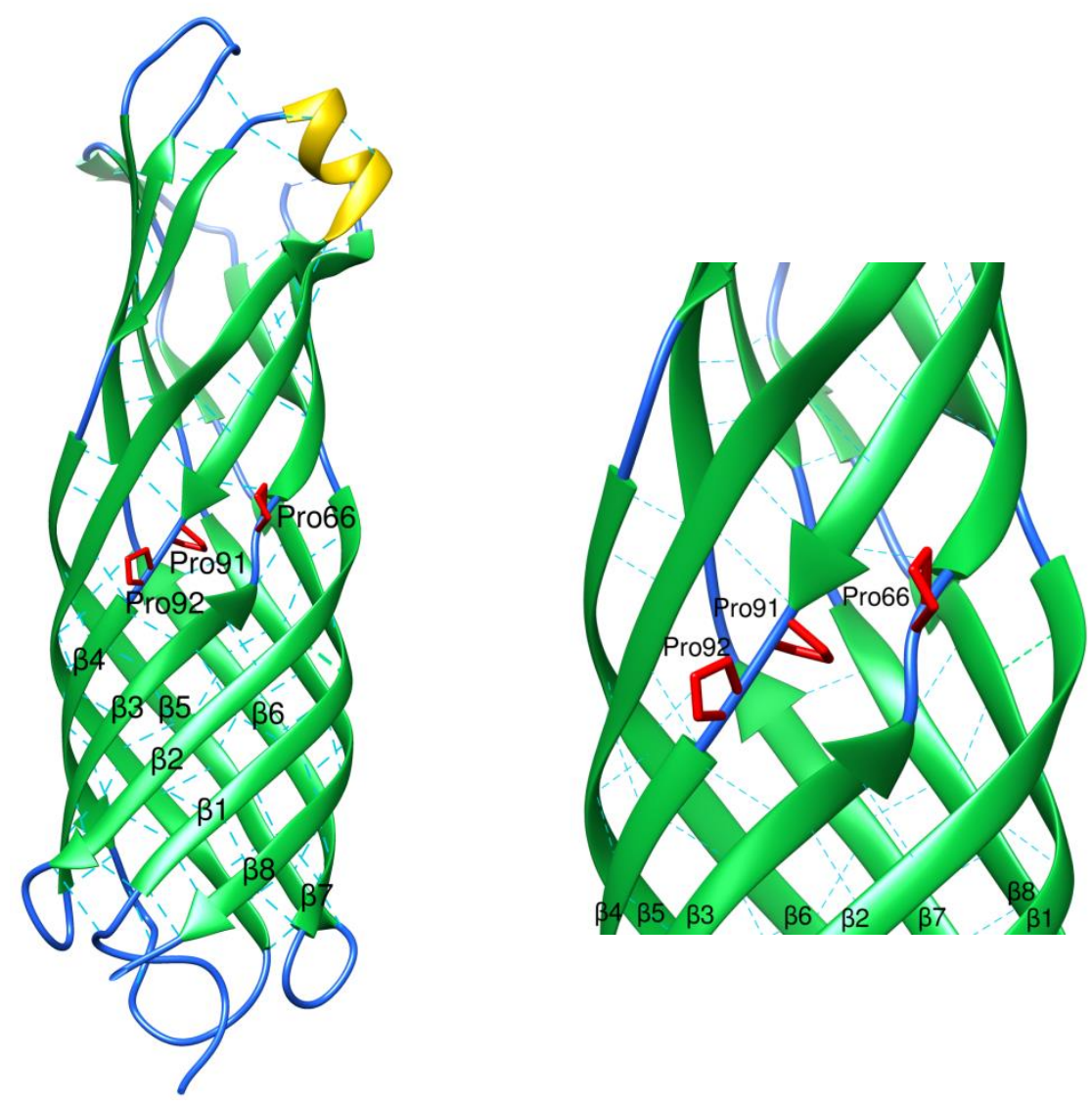

Figure 10. Crystal structure of Pseudomonas aeruginosa OprG (PDB code 2X27). $\beta$ strands are colored green, $\alpha$-helix is colored yellow, loops are blue and prolines around the proposed hypothetical lateral gate are colored red. The numbering of the residues was changed by +1 (for artificial leading methionine) to match the numbering used in this paper. The figure was prepared with UCSF Chimera.

for the FadL-mediated transport of fatty acids in E. coli $[86,87]$. However, the transport of apolar compounds by OprG has so far not been demonstrated.

To investigate the substrate specificity of OprG and to test the lateral gate hypothesis of apolar substrate release into the lipid bilayer, we conducted comprehensive liposome-swelling and bacterial growth assays with a large panel of 
potential substrates. Despite previous claims that OprG might function as a transporter for hydrophobic compounds, we discovered that it forms a channel for the transport and uptake of small amino acids including glycine, alanine, valine, serine, and threonine. The mutation of the conserved Pro92 to an alanine resulted in a loss of the transport function, while mutations of Pro66 and Pro91 affected the ability of the protein to insert into membranes. In order to obtain insight into the mechanistic and structural roles of these conserved prolines, we solved by NMR spectroscopy the dynamic structure of the P92A mutant of OprG and compared it with the dynamic structure of wild-type OprG in dihexanoyl-phosphatidylcholine (DHPC) micelles. We found that the loops are dynamically disordered in the mutant and wild-type proteins, but that the height of the barrel wall and the loop dynamics are much more asymmetric in the mutant than in wild-type OprG. Possible roles for the function of the proline-rich cluster of OprG to facilitate transport of small amino acids across the OM are discussed.

\subsection{Methods.}

2.2.1 Expression and Purification of OprG in Escherichia coli. The P. aeruginosa strain PA01 OprG expression construct was designed without the $\mathrm{N}$-terminal signal sequence, i.e. residues 1 to 26 were replaced with Met1 so that His27 becomes His2 in

our numbering system. The expression constructs contained a C-terminal 6xHis-tag and was cloned into a pET30a vector containing the T7 promoter. E. coli strain BL21 (DE3) 
competent cells were transformed with the OprG containing plasmid. One colony from the transformation was used to inoculate $20 \mathrm{ml}$ of LB or minimal media $\left(\mathrm{D}_{2} \mathrm{O}(99.99 \%)\right.$, 2 g/liter ${ }^{2} \mathrm{H}(98 \%)-{ }^{13} \mathrm{C}(99 \%)$-labeled D-glucose, $1 \mathrm{~g} /$ liter ${ }^{15} \mathrm{~N}(98 \%)$-labeled $\left(\mathrm{NH}_{4}\right)_{2} \mathrm{SO}_{4}$, and $1 \%{ }^{2} \mathrm{H}-,{ }^{13} \mathrm{C}-,{ }^{15} \mathrm{~N}$-labeled Bioexpress (Cambridge Isotope Laboratories)). This preculture was grown overnight at $37^{\circ} \mathrm{C}(225 \mathrm{rpm}$ shaker speed). The $20 \mathrm{ml}$ preculture was pelleted at $25^{\circ} \mathrm{C}$ for $10 \mathrm{~min}$ at $4000 \mathrm{rpm}$ and resuspended in $1 \mathrm{~L}$ of LB or minimal media. $1 \mathrm{~L}$ cultures were grown at $37^{\circ} \mathrm{C}$ to an optical density of 0.5 . After addition of $1 \mathrm{mM}$ isopropyl $\beta$-D-1-thiogalactopyranoside the culture was incubated at $37^{\circ} \mathrm{C}$ for 4 hours. Cells were pelleted at $6000 \mathrm{rpm}$ and $4^{\circ} \mathrm{C}$ for $15 \mathrm{~min}$ and stored at $-80^{\circ} \mathrm{C}$ or immediately purified.

The cell pellet containing OprG was resuspended on ice in $10 \mathrm{ml}$ Buffer A (50 $\mathrm{mM} \mathrm{NaPO}_{4}$ at $\mathrm{pH} 8.0,300 \mathrm{~mm} \mathrm{NaCl}$ ) supplemented with $25 \%$ sucrose and $100 \mu \mathrm{L}$ Pierce \#78415 protein inhibitor cocktail. The resuspended cell pellet was lysed with three passes through a microfluidizer MP-110P from microfluidics NewtOprGon, MA at 20,000 psi. The soluble cell fraction was separated from the inclusion bodies by centrifugation at $11,000 \times \mathrm{g}$ for $15 \mathrm{~min}$ at $4^{\circ} \mathrm{C}$. The supernatant was removed and inclusion bodies were washed three times by resuspension in $10 \mathrm{ml}$ Buffer $A$ and centrifugation at $11,000 \times \mathrm{g}$ for $15 \mathrm{~min}$ at $4^{\circ} \mathrm{C}$. The last resuspension was done in $10 \mathrm{ml}$ solubilization Buffer $\mathrm{B}$ (50 $\mathrm{mM} \mathrm{NaPO}_{4}$ at $\mathrm{pH} 8.0,300 \mathrm{~mm} \mathrm{NaCl}, 8 \mathrm{M}$ urea, $20 \mathrm{~mm}$ imidazole) followed by rotation at $4^{\circ} \mathrm{C}$ overnight. The solubilized inclusion bodies were separated from cell debris by 
centrifugation at $27,000 \times \mathrm{g}$ for $20 \mathrm{~min}$ at $4^{\circ} \mathrm{C}$. The supernatant was rotated at $4^{\circ} \mathrm{C}$ for 1 hr with $5 \mathrm{ml}$ of prewashed Ni-NTA agrose beads (Qiagen). The Ni-NTA column was washed with $50 \mathrm{ml}$ of Buffer B. OprG was eluted with six $5 \mathrm{ml}$ fractions of Buffer B supplemented with $250 \mathrm{~mm}$ imidazole and stored at $4^{\circ} \mathrm{C}$. All elution fractions were pooled and concentrated to $1 \mathrm{ml}$ using Ultra15 10,000 MWCO membranes (Millipore, CA).

2.2.2 Site-directed mutagenesis. Primers were designed to mutate prolines 66 , 91, and 92 to alanines and to generate the double mutant PP91/92AA. A Stratagene QuikChange Site-Directed Mutagenesis Kit \# 200518 was used to make point mutations to the wild type OprG. Parent DNA coding for OprG and the forward and reverse primers of each desired mutation were cycled 20 times in a PCR reaction where the annealing step was set to $68^{\circ} \mathrm{C}$. Amplification products were digested for 1 hour with DpnI. DNA was transformed into XL1-Blue supercompetent cells \#200236 from Agilent Technologies. Transformation guidelines were followed as described in the QuikChange mutagenesis kit. Cells were plated and incubated overnight at $37^{\circ} \mathrm{C}$. One colony was selected for a $6 \mathrm{~mL}$ overnight culture shaken at $225 \mathrm{rpm}$ and $37^{\circ} \mathrm{C}$. The Qiagen QIAprep Miniprep kit was used to purify DNA for sequence analysis. Successfully mutated DNA was stored at $-20^{\circ} \mathrm{C}$.

2.2.3 Refolding and Sample Preparation. The refolding of wild-type and mutant OprG into DHPC micelles followed the protocol previously described for E. coli OprH 
[92]. $1 \mathrm{~mL}$ of 0.4 to $0.6 \mathrm{mM}$ OprG was added dropwise to $9 \mathrm{ml}$ refolding buffer (20 $\mathrm{mM}$ Tris- $\mathrm{HCl}$ at $\mathrm{pH} 8.5,5 \mathrm{mM}$ EDTA, and $0.6 \mathrm{mM}$ L-arginine, 3\% (wt/vol) DHPC) and incubated at $37^{\circ} \mathrm{C}$ for 72 hours. OprG exhibited a gel shift from about $29 \mathrm{kDa}$ to about $24 \mathrm{kDa}$ upon refolding in DHPC. The solution was concentrated and the buffer was exchanged by dilution/concentration to $25 \mathrm{mM} \mathrm{NaPO}_{4}$ at $\mathrm{pH} \mathrm{6.0,} \mathrm{containing} 50 \mathrm{mM} \mathrm{KCl}$, $0.05 \% \mathrm{NaN}_{3}$, and $5 \% \mathrm{D}_{2} \mathrm{O}$. The final NMR samples were concentrated to 1.0 to $1.5 \mathrm{mM}$ OprG.

2.2.4 Selective Amino-Acid Labeling. OprG versions with selectively ${ }^{15} \mathrm{~N}$-labeled Phe and lle residues were expressed in CT19 cells [108]. Cells were grown in $1 \mathrm{~L}$ minimal media supplemented with the $0.2 \mathrm{~g}$ of the respective ${ }^{15} \mathrm{~N}$-labeled amino acid. Selectively labeled versions of P92A OprG with ${ }^{15} \mathrm{~N}$-labeled Asp, Phe, Val, Leu, and lle were prepared. The purification and refolding protocols were identical to those used for the uniformly ${ }^{15} \mathrm{~N}$-labeled OprG samples. Typically, ${ }^{1} \mathrm{H}-{ }^{15} \mathrm{~N}$-TROSY spectra were recorded for each of selectively labeled proteins to obtain additional assignments or to resolve ambiguities of some assignments.

2.2.5 Liposome swelling assay. The assay was performed as described previously [109] with the following modifications. Liposomes were made by suspending a dried film of $3 \mu$ moles egg phosphatidylcholine (Avanti Polar Lipids, Alabaster, Al) and $0.15 \mu$ moles egg L- $\alpha$-phosphatidic acid (Avanti Polar Lipids, Alabaster, Al) in $20 \mu \mathrm{L}$ of a $1.875 \mathrm{mM}$ micellar solution of $n$-dodecylphosphocholine (Anatrace, Maumee, $\mathrm{OH}$ ) in water 
containing 2 (protein-to-lipid ratio 1:1575) or 6 (protein-to-lipid ratio 1:525) nmoles OprG, sonicated for $15 \mathrm{~min}$, and completely dehydrated in vacuo. After rehydration in assay buffer (12 mM stachyose, 10 mM HEPES-NaOH, pH 7.5) under vigorous shaking and 2 times 15 min sonication, a suspension of multilamellar vesicles containing OprG in their membranes was obtained. Reconstitution of OprG was quantitative as far as detectable on Coomassie stained SDS-PAGE gels (data now shown). In a 96 well plate (Corning \#3631), $5 \mu \mathrm{L}$ OprG liposomes were diluted with $200 \mu \mathrm{L}$ of an equiosmolar analyte solution in $10 \mathrm{mM}$ HEPES-NaOH, $\mathrm{pH} 7.5$, and absorbance was monitored at $405 \mathrm{~nm}$ using a Perkin-Elmer HTS 7000 plate reader over a period of $5 \mathrm{~min}$. Data was analyzed following published procedures $[109,110]$ and swelling rates were reported relative to L-alanine.

2.2.6 Growth assays. The wild-type PA01 (H899) and oprG-deficient H900 strains of $P$. aeruginosa [73] were kind gifts from Dr. Robert Hancock (University of British Columbia, Vancouver, Canada). Growth assays with wild-type PA01 and mutant H900 strains were performed following a described protocol [105] with some small changes. The bacteria were grown overnight in BM2 media supplemented with several desired nitrogen sources as indicated in Results. The overnight cultures were sub-cultured to a starting optical density of 0.01 at $600 \mathrm{~nm}$ in pre-warmed fresh BM2 media containing the specific nitrogen sources. These cultures were grown at $37^{\circ} \mathrm{C}$ in an orbital shaker shaking at $250 \mathrm{rpm}$. To ensure sufficient aeration, $50 \mathrm{ml}$ cultures were grown in 1-L 
flasks. Aliquots of the cultures were taken at specified time intervals and the turbidity at $600 \mathrm{~nm}$ was determined using a spectrophotometer.

2.2.7 NMR Spectroscopy. All NMR experiments were recorded at $45{ }^{\circ} \mathrm{C}$ on a Bruker Avance III 800 spectrometer equipped with a triple-resonance cryoprobe. All double- and triple-resonance experiments were performed using the Bruker Topspin version 2.1.6 software suite. Sequential backbone assignments were obtained by recording TROSY versions of HNCA, HNCB, HNCO and HNCACO experiments. ${ }^{15} \mathrm{~N}$-edited ${ }^{15} \mathrm{~N}-{ }^{1} \mathrm{H}-{ }^{1} \mathrm{H}$ NOESY-TROSY and ${ }^{15} \mathrm{~N}-{ }^{1} \mathrm{H}-{ }^{15} \mathrm{~N}$ HSQC-NOESY-HSQC experiments with mixing times of $200 \mathrm{~ms}$ were recorded to obtain nuclear Overhauser effects (NOEs). $T_{1}, T_{2}$, and $\left({ }^{1} \mathrm{H}\right)-{ }^{15} \mathrm{~N}$ heteronuclear NOEs were measured using two-dimensional ${ }^{15} \mathrm{~N}-{ }^{1} \mathrm{H}$ TROSYbased experiments. NMR data were processed with NMRPipe and analyzed with Sparky software [111].

2.2.8 H/D Exchange Experiments. ${ }^{15} \mathrm{~N}$-labeled NMR samples of refolded wildtype and P92A OprG were dried overnight by lyophilization. $\mathrm{D}_{2} \mathrm{O}$ was added to $\sim 1 \mathrm{mM}$ protein, the samples were mixed by vortexing, and ${ }^{15} \mathrm{~N}$-TROSY spectra were collected after 20 min, 1 hour, 4 hours, 1 day and 1 week after $D_{2} \mathrm{O}$ addition. Hydrogen bonds were identified by the presence of corresponding resonances in the ${ }^{15} \mathrm{~N}$-TROSY spectra.

2.2.9 Structure Calculation. Distance constraints were calibrated and calculated manually based upon an average distance of $3.3 \AA$ between $\beta$-strands. Backbone 
dihedral angle constraints were determined from chemical shifts corrected for deuterium and TROSY effects using TALOS+ [112]. Hydrogen bond constraints derived from $\mathrm{H} / \mathrm{D}$ exchange experiments were set at 2.5 and $3.5 \AA$ for $\mathrm{HN}-\mathrm{O}$ and $\mathrm{N}-\mathrm{O}$ distances, respectively. Structure calculations were performed using CNS version 1.2 [113] using 4,000 high temperature, 8,000 torsion slow-cool, and 8,000 Cartesian slow-cool annealing steps. A total of 100 structures were calculated, and the 20 lowest energy structures were selected for ensemble analysis. Ramachandran map analysis was performed with PROCHECK-NMR [114].

2.2.10 Data Deposition. NMR chemical shifts and other data have been deposited in the Biomolecular Magnetic Resonance Data Bank and the structural coordinates of wild-type and P92A OprG have been deposited in the Protein Data Bank under accession codes $2 \mathrm{NGL}$ and $2 \mathrm{~N} 6 \mathrm{P}$, respectively.

\subsection{Results}

2.3.1 OprG expression and refolding. OprG was overexpressed in E. coli with a C-terminal His-tag and with its signal sequence deleted as described in Experimental Procedures. This protein, which expressed into inclusion bodies in high yields, was purified by affinity chromatography in a urea-denatured form and refolded by dilution of the denaturant in the presence of DHPC micelles. Wild-type OprG was quantitatively refolded as seen from the shift of its apparent molecular mass (Fig. 11). To investigate 
the properties of the proline-rich putative lateral gate we mutated prolines 66,91 , and 92 to alanines to obtain the single mutants P66A, P91A, and P92A and the double mutant PP91/92AA. All mutants showed good expression and their refolding was as efficient as that of wild-type OprG (Fig. 11).

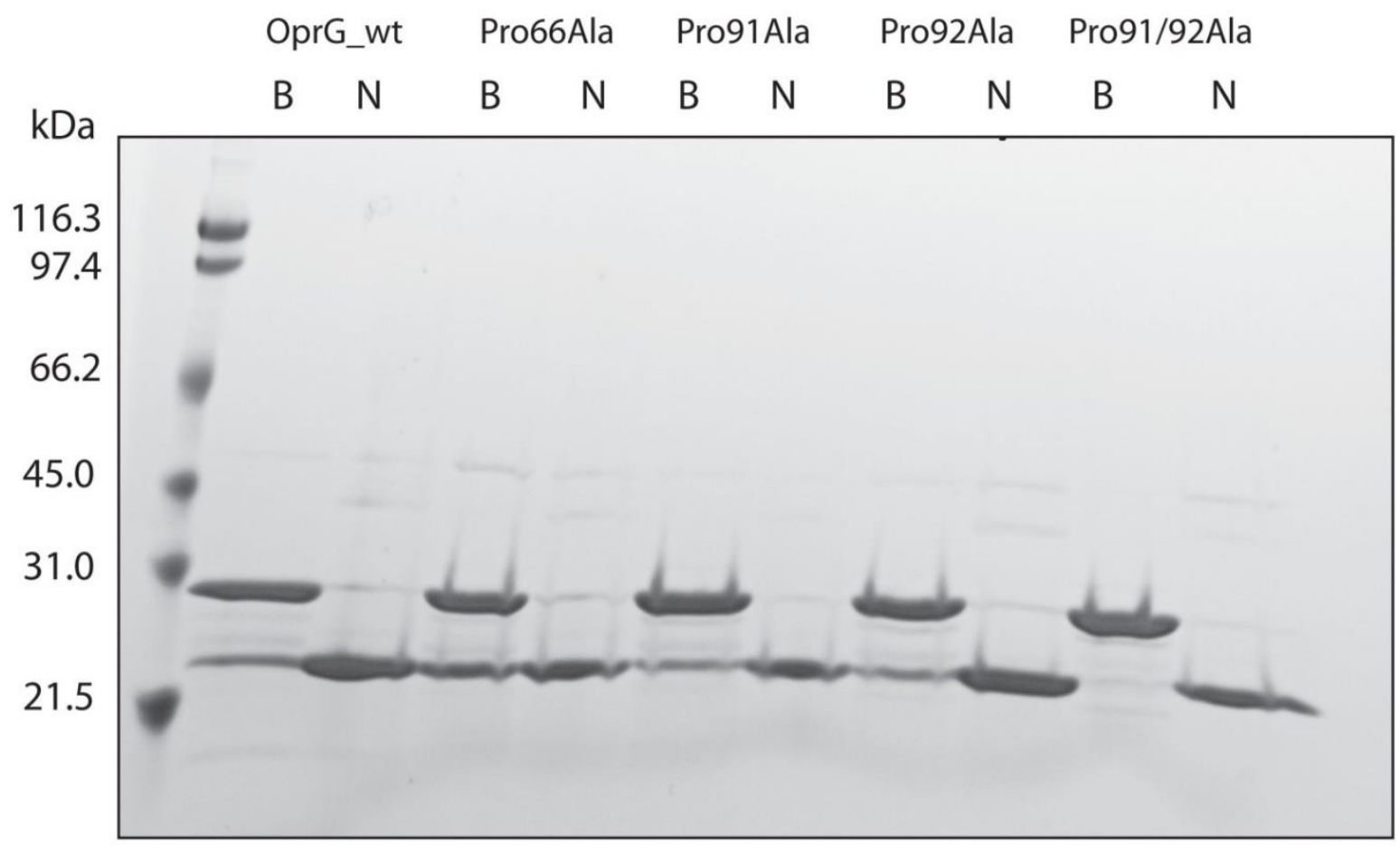

Figure 11. Refolding of OprG and OprG proline mutants into DHPC micelles. SDS-PAGE gel showing boiled (B) and refolded (N) OprG and OprG proline mutants in DHPC micelles. All proteins exhibit a gel shift from about $29 \mathrm{kDa}$ to $24 \mathrm{kDa}$ upon refolding in DHPC.

2.3.2 Liposome-swelling assay. A liposome-swelling assay was used to test the OprG-mediated permeation of small molecules across the liposomal membrane. A wide range of low-molecular weight compounds was tested, including sugars, amino acids, aliphatic alcohols and fatty acids, some other apolar compounds, several common antibiotics, and inorganic salts, but liposome swelling was only observed with the small amino acids glycine, alanine, serine, threonine and valine (Fig. 12, Table 1). The swelling 
assay showed that the OprG-mediated permeation of alanine (relative rate 1.00) was most efficient and that of valine (0.43) was least efficient, while threonine (0.77), glycine (0.82), and serine (0.53) exhibited intermediate permeation rates. No liposome swelling was observed in the absence of OprG and, hence, these same amino acids do not permeate through pure lipid bilayers (black squares in Fig. 12). Although the P92A mutant was well folded and readily incorporated into liposomes as demonstrated by SDS-PAGE, it did not facilitate the permeation of any of the tested substrates into the liposomes (blue triangles in Fig. 12). The P66A, P91A and PP91/92AA mutants refolded
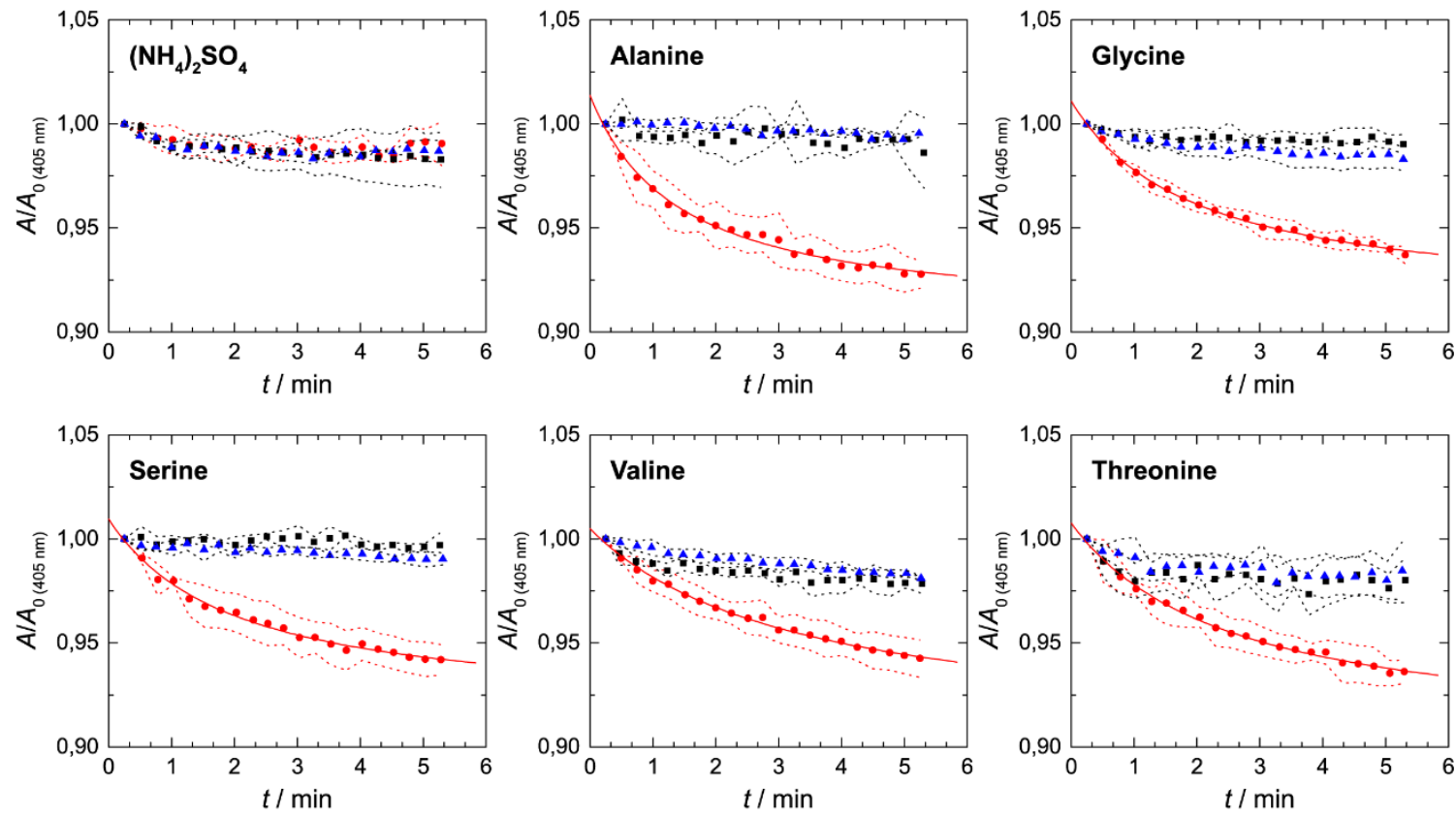

Figure 12. Liposome-swelling assay of OprG. Liposome-swelling assay with reconstituted wild-type (red dots) and P92A OprG (blue triangles) in the presence of ammonium sulfate and various amino acids. Substrate uptake was observed for alanine, glycine, valine, serine, and threonine, but not for $\left(\mathrm{NH}_{4}\right)_{2} \mathrm{SO}_{4}$. Pure lipid liposomes (black squares) were used as controls. Data is presented as mean \pm SD (dashed line) of at least three independent preparations. Solid lines denote fits of theoretical swelling function where applicable. See also Table 1. 
well in DHPC, but these proteins did not insert in sufficient amounts into the liposomes. Therefore, these mutants could not be tested for amino acid permeation using the liposome-swelling assay.

Table 1. Compounds tested in liposome-swelling assay that did not produce any observable swelling.

Inorganic salts $\mathrm{NaCl}, \mathrm{KCl}, \mathrm{Na}_{2} \mathrm{SO}_{4},\left(\mathrm{NH}_{4}\right)_{2} \mathrm{SO}_{4}$

Saccharides L-Arabinose, D-Glucose, Sucrose, Maltose, Trehalose, Melezitose, Stachyose

Amino acids L-Arginine, L-Lysine, L-Glutamate, L-Methionine, L-Proline

2.3.3 Cell growth. To confirm the results of the liposome-swelling assay, we tested the growth of $P$. aeruginosa with wild-type OprG and a strain of $P$. aeruginosa (H900), in which the oprG-gene was disrupted, under conditions where the selected small amino acids provided the only sources of nitrogen. P. aeruginosa can utilize alanine as the source of nitrogen and carbon, but glycine, valine, serine and threonine can be used as sources of nitrogen only [115]. To this end, we measured cell growth in minimal media that were supplemented with the amino acids of interest at 2-50 mM concentrations. Optimal substrate concentrations, at which the loss of OprG would compromise growth most, were determined for each amino acid. Although the growth kinetics in these cultures were somewhat variable from day to day, differences between OprG+ and OprG- strains were always observed as reported. Representative growth curves of three separate experiments for each tested amino acid are shown in Fig. 13. The difference in growth between OprG+ and OprG- strains appears greatest for glycine 

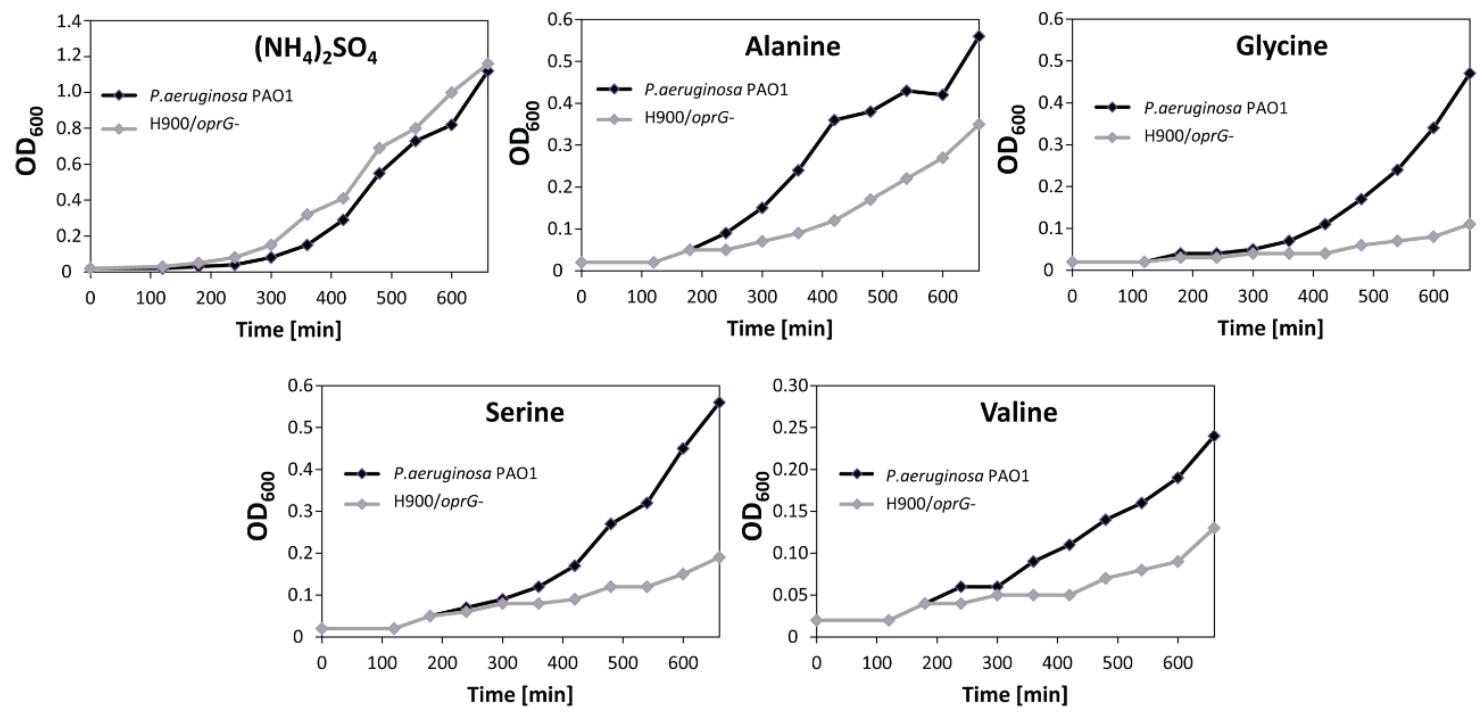

Figure 13. Growth phenotypes of $\boldsymbol{P}$. aeruginosa PAO1 and an OprG- mutant. Cells were grown in BM2 minimal media supplemented with putative substrates of OprG as the sole nitrogen sources. The nitrogen sources include $10 \mathrm{mM}\left(\mathrm{NH}_{4}\right)_{2} \mathrm{SO}_{4}$ (control), $2 \mathrm{mM}$ alanine, $10 \mathrm{mM}$ glycine, $10 \mathrm{mM}$ serine, or $50 \mathrm{mM}$ valine. Data shown are representatives of at least three independent experiments.

and serine and a little less pronounced for valine and alanine. However, in the wild-type strain, growth is most efficient when alanine is used as the single nitrogen source, which is consistent with alanine permeating best through OprG in the liposome-swelling assay (Table 2). No growth defect of the OprG- strain was observed when ammonium sulfate was used as the sole source of nitrogen, confirming that the facilitation of uptake of the tested amino acids by OprG is physiological. Growth on threonine was extremely slow, even when threonine was supplied in concentrations greater than $50 \mathrm{mM}$. Therefore, threonine may not be a physiological substrate for OprG-mediated permeation although utilization of threonine as a sole nitrogen source could also be metabolically inefficient. 
Table 2. Doubling times of $P$. aeruginosa strains (PAO1 and H900/oprG-) grown in BM2 medium with amino acids as sole sources of nitrogen. Results shown are the means of three independent experiments.

\begin{tabular}{|ccc|}
\hline Substrate & \multicolumn{2}{c|}{ Average doubling time [min] } \\
& P. aeruginosa PAO1 & H900/oprG- \\
\hline$\left(\mathrm{NH}_{4}\right)_{2} \mathrm{SO}_{4}(10 \mathrm{mM})$ & 109 & 91 \\
Alanine $(2 \mathrm{mM})$ & 85 & 148 \\
Glycine $(10 \mathrm{mM})$ & 116 & 207 \\
Serine $(10 \mathrm{mM})$ & 151 & 220 \\
Valine $(50 \mathrm{mM})$ & 188 & 235 \\
\hline
\end{tabular}

2.3.4 Solution structures of wild-type and P92A OprG in DHPC micelles. To

determine the solution structures of wild-type and P92A OprG, ${ }^{2} \mathrm{H},{ }^{13} \mathrm{C},{ }^{15} \mathrm{~N}$-labeled proteins were overexpressed in E. coli, purified, and refolded in DHPC micelles as described in Experimental Procedures. The ${ }^{15} \mathrm{~N}-{ }^{1} \mathrm{H}$ TROSY-HSQC spectrum of ${ }^{2} \mathrm{H}-,{ }^{13} \mathrm{C}-$ ,${ }^{15} \mathrm{~N}$-labeled P92A wild-type OprG shown in Fig. 14 displayed good dispersion in the proton dimension from 8.5 to $9.5 \mathrm{ppm}$, indicating well-formed secondary structure. A larger number of cross-peaks overlapped in the 7.8 to $8.5 \mathrm{ppm}$ region suggesting the additional presence of significant regions of disordered residues in the protein. OprG also showed very high quality TROSY spectra and a ${ }^{2} \mathrm{H},{ }^{13} \mathrm{C},{ }^{15} \mathrm{~N}$-labeled sample was used for assignment and structure determination (Fig. 15 D and Fig. 16). However, the ${ }^{15} \mathrm{~N}-{ }^{1} \mathrm{H}$ TROSY spectra of the other ${ }^{15} \mathrm{~N}$-labeled proline mutant samples were of substantially poorer quality. In the case of P66A, only a few high-field peaks of very low intensity were visible (Fig. 15 B). Analysis of the TROSY spectrum of P91A revealed no visible high-field peaks, even when increasing the contour scale (Fig. 15 C). Compared to P92A (Fig. 16), 


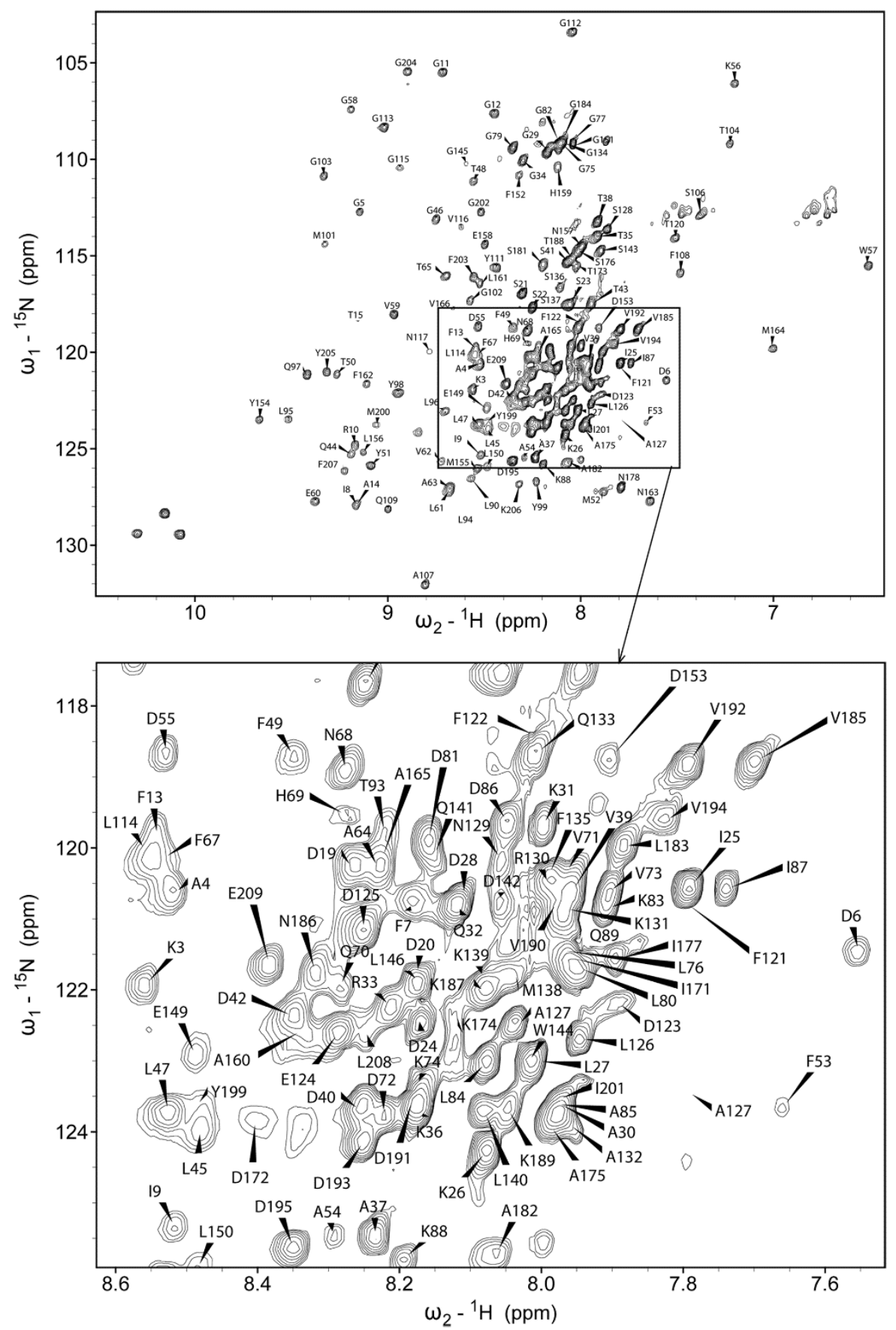

Figure 14. ${ }^{15} \mathrm{~N}-{ }^{1} \mathrm{H}$ TROSY spectrum of ${ }^{2} \mathrm{H}-,{ }^{13} \mathrm{C}-,{ }^{15} \mathrm{~N}$-labeled OprG in DHPC micelles collected at $800 \mathrm{MHz}$ and $45{ }^{\circ} \mathrm{C}$. The refolded protein sample was exchanged into 25 $\mathrm{mm} \mathrm{NaPO}_{4}$ at $\mathrm{pH} 6.0,50 \mathrm{~mm} \mathrm{KCl}, 0.05 \% \mathrm{NaN}_{3}$, and $5 \% \mathrm{D}_{2} \mathrm{O}$ before being concentrated to $\sim 1.0 \mathrm{mM}$ for NMR experiments. 
only approximately 40 cross-peaks were present in the PP91/92AA spectrum (Fig. 15 E). In order to assign the $\mathrm{H}, \mathrm{N}, \mathrm{C} \alpha, \mathrm{C} \beta$, and $\mathrm{CO}$ resonances TROSY-based triple resonance NMR experiments were performed on the ${ }^{2} \mathrm{H}-,{ }^{13} \mathrm{C}-,{ }^{15} \mathrm{~N}$-labeled wild-type and P92A samples to establish their through-bond connectivities. For wild-type HNCA, HN(CO)CA, $\mathrm{HN}(\mathrm{CA}) \mathrm{CB}, \mathrm{HN}(\mathrm{COCA}) \mathrm{CB}, \mathrm{HNCO}$, and $\mathrm{HN}(\mathrm{CA}) \mathrm{CO}$ and for P92A HNCA, HN(CO)CA, HNCB and HNCO spectra were collected. To help the assignment process we also prepared specifically ${ }^{15} \mathrm{~N}$-labeled Phe and lle samples of wild-type and specifically ${ }^{15} \mathrm{~N}$-labeled Asp,
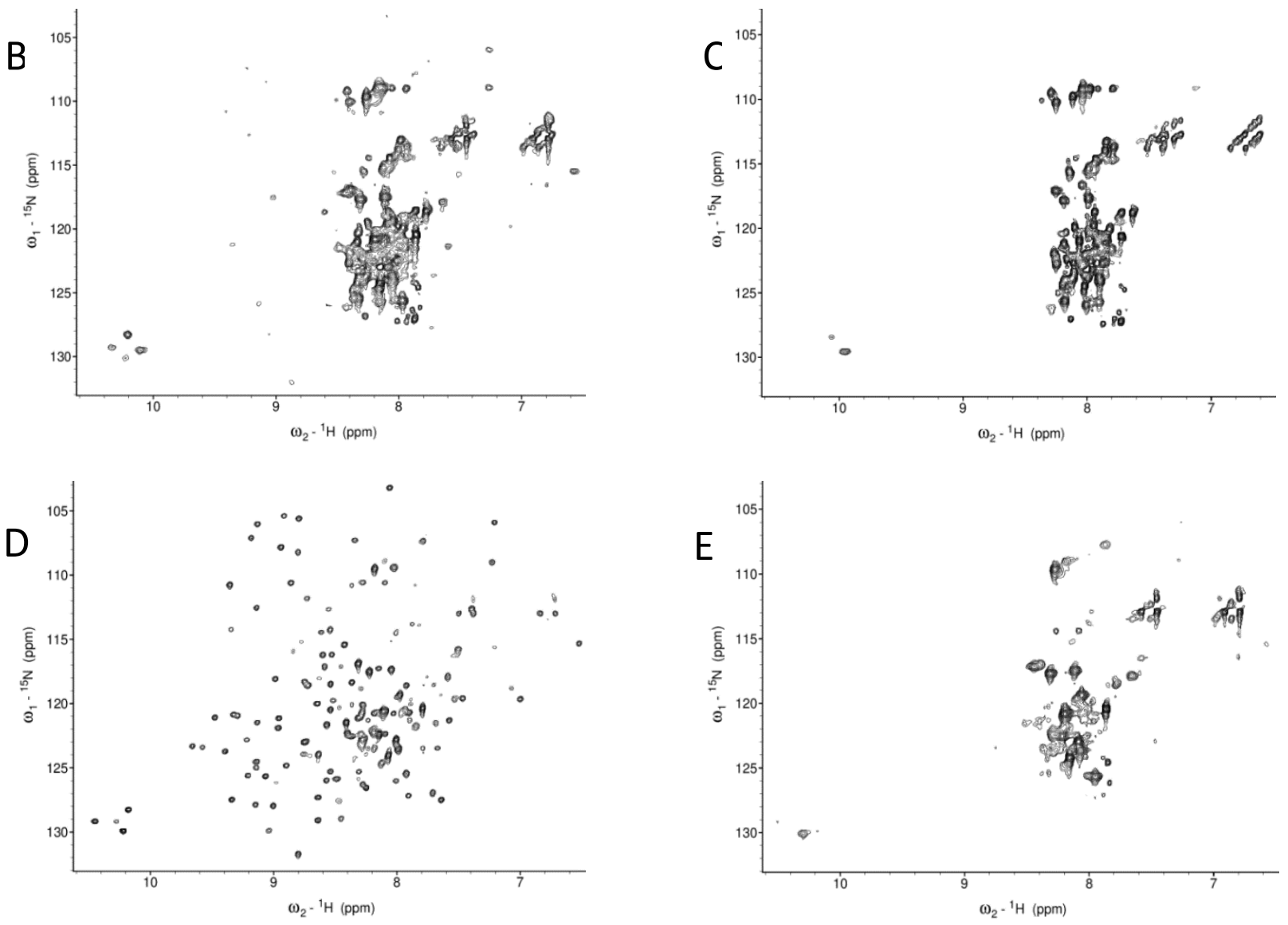

Figure 15. ${ }^{15} \mathrm{~N}$-1 $\mathrm{H}$ TROSY spectra of ${ }^{15} \mathrm{~N}$-labeled OprG proline mutants in DHPC micelles collected at $800 \mathrm{MHz}$ and $45^{\circ} \mathrm{C}$. B, P66A OprG; C, P91A OprG; D, P92A OprG; E, PP91/92AA OprG. The refolded protein samples were exchanged into $25 \mathrm{mM} \mathrm{NaPO}_{4}$ at $\mathrm{pH} 6.0,50 \mathrm{mM} \mathrm{KCl} 0.05 \% \mathrm{NaN}_{3}$, and $5 \% \mathrm{D}_{2} \mathrm{O}$ before being concentrated to $\sim 1.0 \mathrm{mM}$ for NMR experiments. 
Phe, Val, Leu, and Ile samples of P92A OprG. Using this approach, $90 \%$ (77\%) of the $\mathrm{N}$ and $\mathrm{H}, 93 \%(81 \%)$ of the $\mathrm{C} \alpha, 92 \%(81 \%)$ of the $C \beta$, and $92 \%(81 \%)$ of the $\mathrm{CO}$ resonances of wild-type (and P92A) OprG were assigned. The larger numbers of missing resonances in P92A are presumably due to more line broadening by intermediate exchange in P92A compared to wild-type. Several residues, which showed weak cross-peaks in the wildtype were missing in the P92A ${ }^{15} \mathrm{~N}-{ }^{1} \mathrm{H}$ TROSY spectrum, including $\mathrm{A} 14, \mathrm{~T} 15, \mathrm{G} 34-\mathrm{K} 36$, T43, N68-K74, G77, G79, G82-L90, E124, S128, N129, K139-Q141, K174-S176, S181, T188, K89, V194, D195, 1201. The lack of these resonances gives the impression of a better dispersion in the 6.8 to $7.5 \mathrm{ppm}$ region of the P92A compared to the wild-type spectrum. On the other hand, several residues that remained unassigned in wild-type could be assigned in P92A. These include Y118, T119, L146-G148, W167-I171. In summary, complete backbone chemical shift assignments were obtained for 181 of the 209 residues of wild-type OprG. Partial assignments were obtained for 14 additional residues, including 6 of the 7 prolines. 14 residues remained unassigned. In the case of P92A, complete backbone assignments were obtained for 152 residues, partial assignments for 19 residues including 4 of the 7 prolines, and 38 residues remained unassigned.

Fig. 17 shows the secondary $C \alpha$ and $C \beta$ chemical shifts along the sequences of both proteins. Eight distinct regions of large negative values typical of $\beta$-strands [116] are clearly evident in both cases. These eight putative $\beta$-strands are separated by regions 

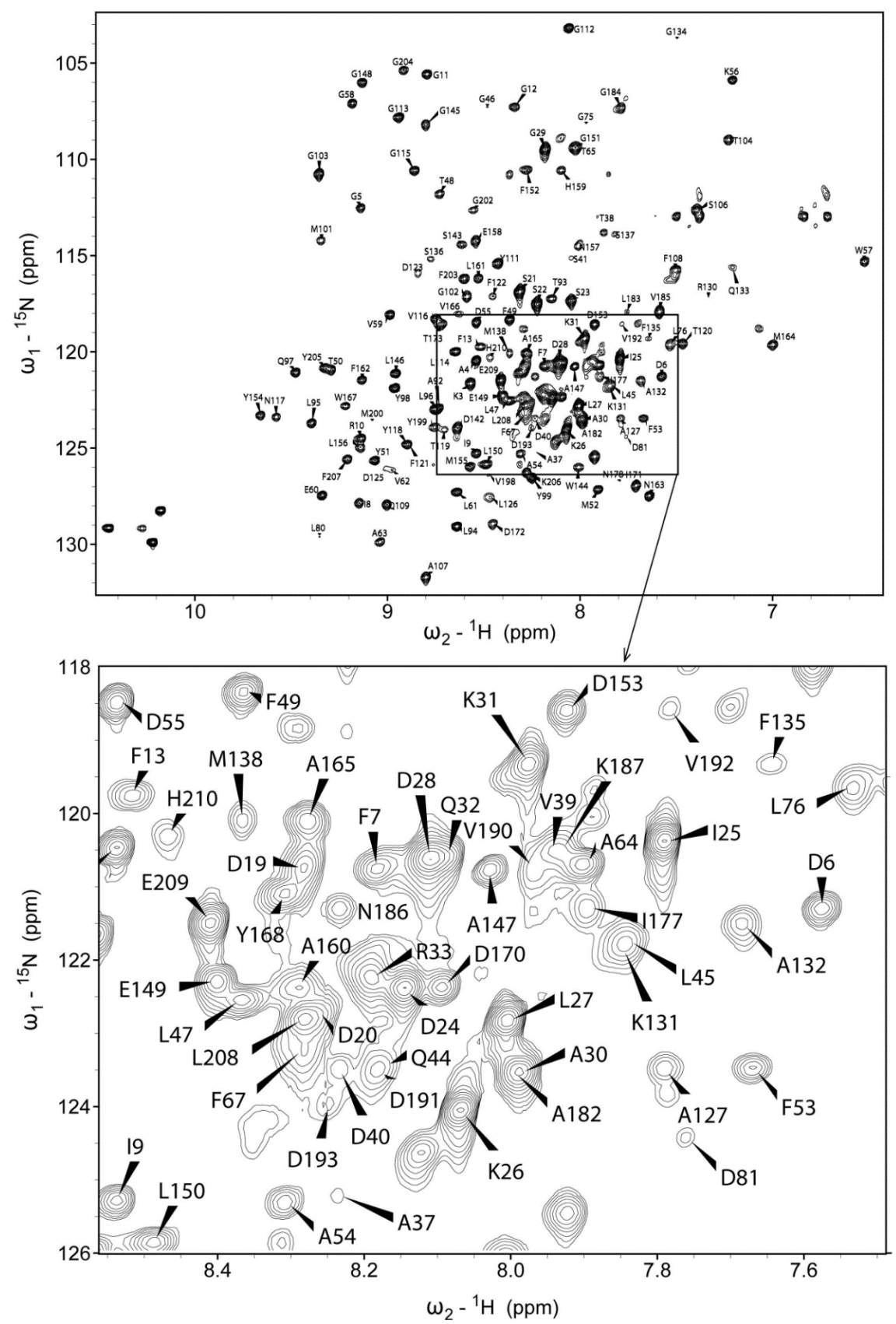

Figure $16,{ }^{15} \mathrm{~N}-{ }^{1} \mathrm{H}$ TROSY spectrum of ${ }^{2} \mathrm{H}-,{ }^{13} \mathrm{C}-,{ }^{15} \mathrm{~N}$-labeled OprG_P92A in DHPC micelles collected at $800 \mathrm{MHz}$ and $45{ }^{\circ} \mathrm{C}$. The refolded protein sample was exchanged into 25 $\mathrm{mM} \mathrm{NaPO}_{4}$ at $\mathrm{pH} 6.0,50 \mathrm{mM} \mathrm{KCl}, 0.05 \% \mathrm{NaN}_{3}$, and $5 \% \mathrm{D}_{2} \mathrm{O}$ before being concentrated to $\sim 1.0 \mathrm{mM}$ for NMR experiments. 

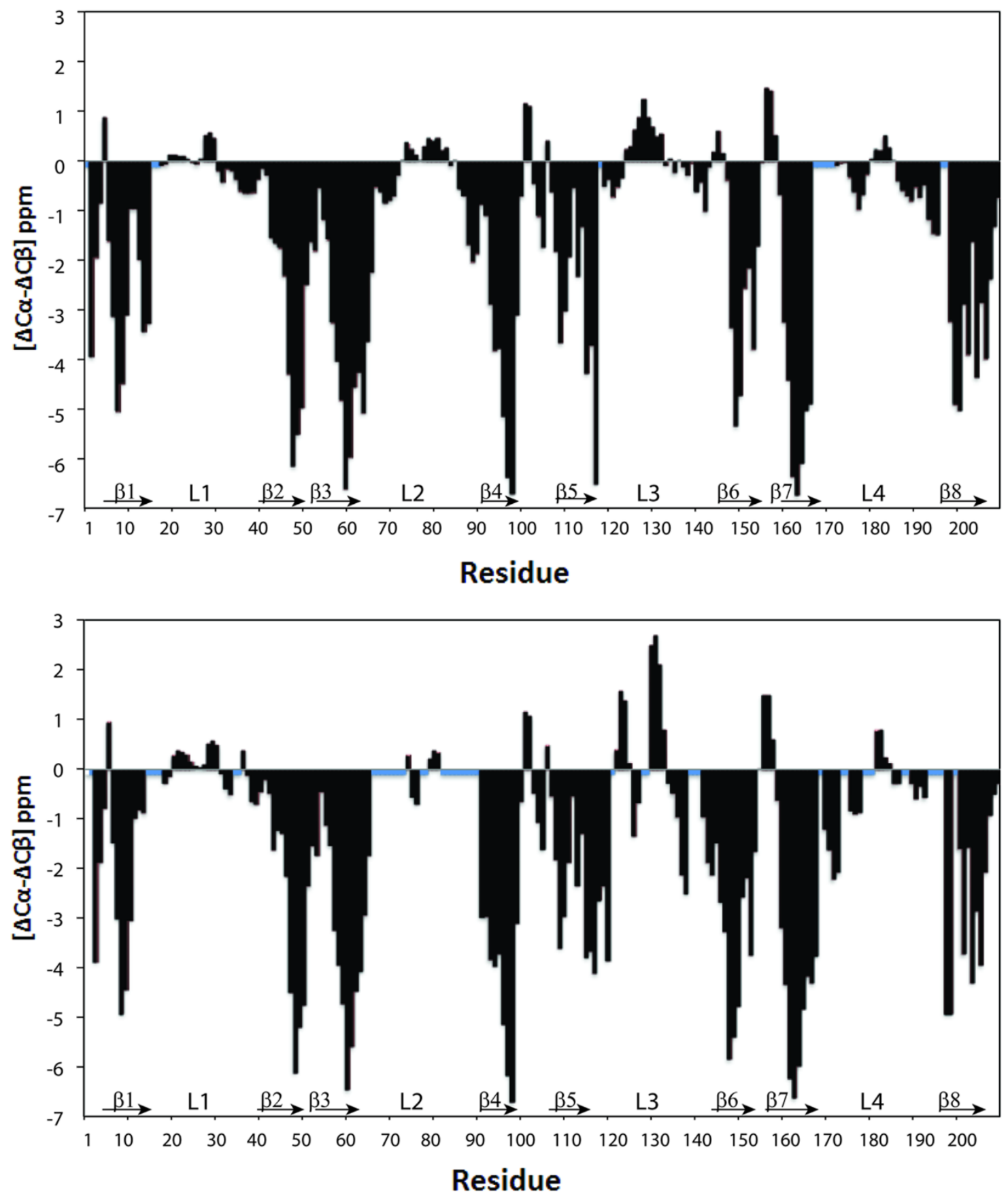

Figure 17. Three-bond averaged secondary chemical shifts of OprG (A) and P92A OprG (B) in DHPC micelles. The secondary chemical shifts, where the deviation $(\Delta)$ of each residue-specific $\mathrm{C} \alpha$ and $C \beta$ chemical shift from random coil values was determined as $\left(\Delta C^{\alpha}-\Delta C^{\beta}\right)=1 / 3\left(\Delta C^{\alpha}{ }_{i-1}+\Delta C^{\alpha}{ }_{i}+\Delta C^{\alpha}{ }_{i+1}-\Delta C^{\beta}{ }_{i-1}-\Delta C^{\beta_{i}}-\Delta C^{\alpha}{ }_{i+1}\right)$, are plotted as a function of the amino acid sequence. Large negative values indicate $\beta$ sheet secondary structure, while large positive values indicate $\alpha$-helical structure. Blue squares denote unassigned residues. Chemical shifts were corrected for both deuteration and TROSY effects prior to analysis. 

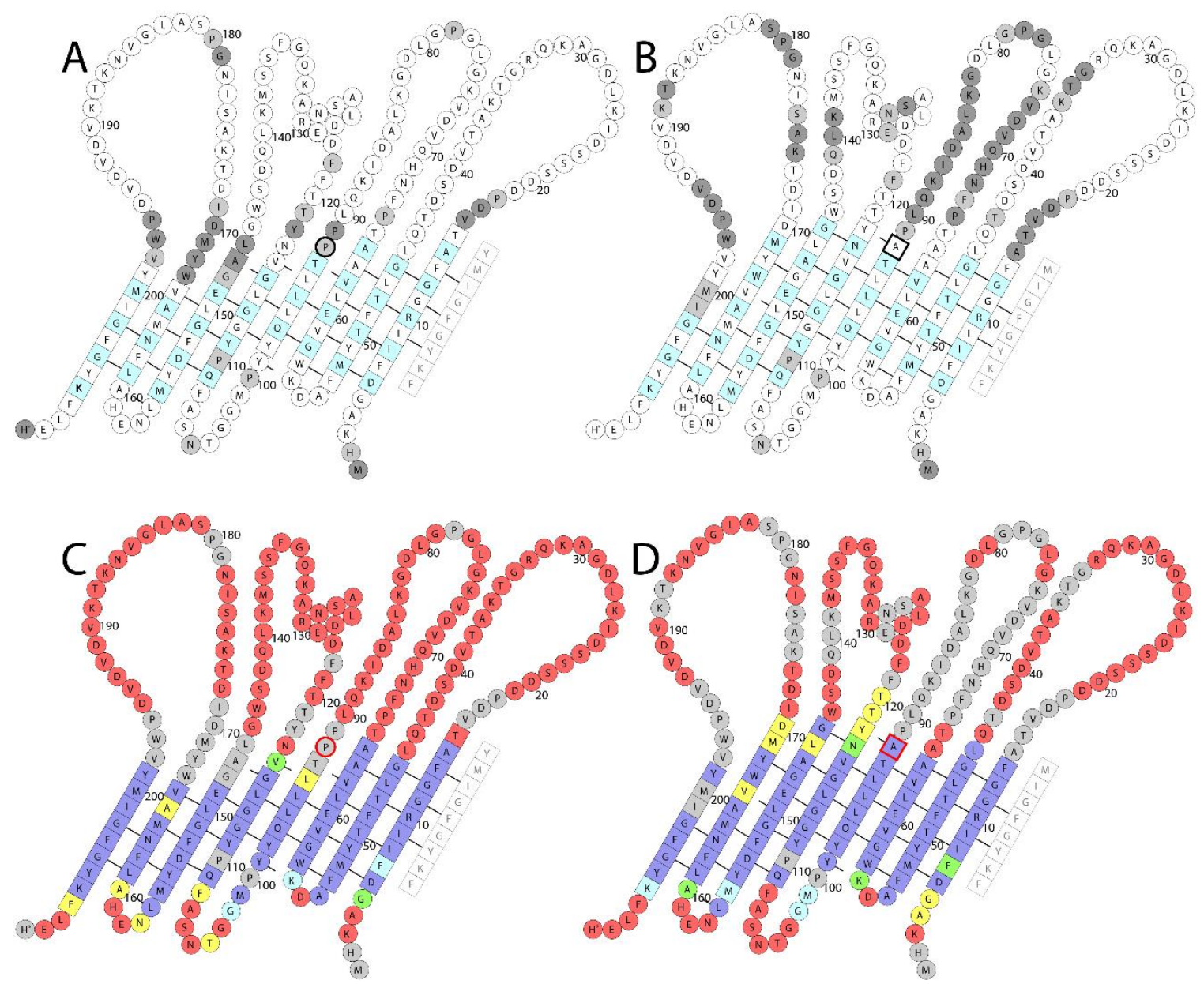

Figure 18. Schematic topologies of OprG (A) and P92A OprG (B) and ${ }^{2} \mathrm{H} /{ }^{1} \mathrm{H}$ exchange experiments of OprG (C) and P92A OprG (D). A and B: Residues that were partially assigned are colored light gray, and residues that were completely unassigned are colored dark gray. For all other residues, complete nitrogen, hydrogen, $\mathrm{C} \alpha, \mathrm{C} \beta$, and $\mathrm{CO}$ assignments were obtained. Residues that face the lumen of the barrel are colored light blue. $\beta$-strand residues are denoted as squares and were determined from the solution structure using the Kabsch and Sander secondary structure algorithm provided with the MOLMOL software package. Loop and turn residues are denoted as circles. Inter-residue lines represent long- and medium-range NOEs observed in NOESY experiments. Hydrogen bond constraints that were identified through ${ }^{2} \mathrm{H} /{ }^{1} \mathrm{H}$ exchange experiments are denoted as black inter-residue lines. $C$ and $\mathbf{D}: \mathrm{D}_{2} \mathrm{O}$ was added to lyophilized protein/DHPC samples and ${ }^{15} \mathrm{~N}-{ }^{1} \mathrm{H}$ TROSY spectra were recorded at $800 \mathrm{MHz}$ and $45^{\circ} \mathrm{C}, 20 \mathrm{~min}, 4$ hours, 1 day, and 1 week after addition of $\mathrm{D}_{2} \mathrm{O}$. Resonances that disappeared before recording the first spectra are colored red, resonances present 20 min after $\mathrm{D}_{2} \mathrm{O}$ addition (but not 1 hour) are colored yellow, resonances present after 4 hours, 1 day, and 1 week are colored green, light blue, and dark blue, respectively. Unassigned residues are colored gray. 
with values around zero that are typical for turns and disordered structures. ${ }^{15} \mathrm{~N}$-edited ${ }^{15} \mathrm{~N}-{ }^{1} \mathrm{H}-{ }^{1} \mathrm{H}$ NOESY-TROSY and ${ }^{15} \mathrm{~N}-{ }^{1} \mathrm{H}_{-}{ }^{15} \mathrm{~N}$ HSQC-NOESY-HSQC experiments were performed to measure NOEs and to generate structural distance constraints. Acquisition of the two types of NOESY spectra helped reduce ambiguities resulting from peak overlaps. For wild-type (P92A) OprG 232 (145) and 263 (154) peaks were assigned and integrated in the ${ }^{15} \mathrm{~N}-{ }^{1} \mathrm{H}-{ }^{1} \mathrm{H}$ NOESY-TROSY and ${ }^{15} \mathrm{~N}-{ }^{15} \mathrm{~N}-{ }^{1} \mathrm{H}$ HSQC-NOESY-HSQC spectra, respectively. Characteristic $\mathrm{HN}-\mathrm{HN}$ inter-strand NOEs identified the antiparallel orientation of the strands and established the closed $\beta$-barrel topology of both proteins. The topologies of the two proteins derived from chemical shifts and observed longrange NOEs leading to inter-strand hydrogen bonds as well as a representation of their assigned residues are summarized in Fig. 18 A and B. In summary, wild-type and P92A OprG displayed similar patterns of long-range NOEs confirming their similar overall $\beta$ barrel structures.

Despite their generally similar topologies, there are significant differences between the two proteins. For example, strong NOEs were observed connecting strands 5 to 7 in the upper part of the P92A barrel that were absent in the wild-type. These include NOEs between residues A92-Y118, L94-V116, N117-G145, G115-A147, G148Y168. Most unassigned residues are found at the barrel-to-loop transitions in the wildtype while they are primarily located in loop 2 and scattered through some of the other loops in P92A. To confirm the presence of inter-strand hydrogen bonds, we performed 
H/D exchange experiments (Fig. $18 \mathrm{C}$ and D). The graded H/D exchange revealed very stable $\beta$-sheets and highly exchangeable loops for both proteins. The H/D exchange experiments also highlight additional differences between wild-type and P92A OprG. Several residues that were unassigned in the wild-type, but assigned in P92A, proved to be resistant to $\mathrm{H} / \mathrm{D}$ exchange with ${ }^{1} \mathrm{H}$ signals persisting for at least 20 min (Y118-T120, L146, M169, D170) or even a week (V116, G145, A147, G148, A165, W167, Y168) after transfer into $\mathrm{D}_{2} \mathrm{O}$ buffer. Some of the additionally assigned residues formed hydrogen bonds between strands 4, 5, 6 and 7 (Fig. $18 \mathrm{C}$ and D). The H/D exchange data combined with inter-strand NOEs allowed us to generate a total of 106 and 116 hydrogen bond constraints for wild-type and P92A OprG, respectively (Fig. 18 A and B).

The structures of wild-type (and P92A) OprG in DHPC micelles were calculated based on 185 (139) NOE-derived distance constraints, 180 (224) chemical shift-derived backbone dihedral angle constraints [117], and 106 (116) hydrogen bond constraints. As expected, the overall folds of the 20 lowest energy conformers were similar for both proteins. Both formed closed eight-stranded $\beta$-barrel structures (Fig. 19 and 20). The barrel backbone root mean square deviations (r.m.s.d.'s) of the NMR ensembles were $0.98 \pm 0.21 \AA$ for wild-type and $0.89 \pm 0.18 \AA$ for the P92A $\beta$-barrels (Table 3). When the periplasmic turns are included, the r.m.s.d.'s are $1.19 \pm 0.28 \AA$ for wild-type and $1.19 \pm$ $0.32 \AA$ for P92A OprG. The average pair-wise r.m.s.d. between the two barrel backbone ensembles is $2.07 \pm 0.41 \AA$. The shear numbers [67] of both $\beta$-barrels are 10 and the $\beta$ - 
strand tilt angles to the membrane normal are $43^{\circ}$. The wild-type $\beta$-barrel has an average length of 7.9 residues and that of P92A 8.6 residues. Despite these overall similar folds, the two structures showed several significant differences in their local hydrogen bonding patterns and $\beta$-barrel lengths. The increased lengths of $\beta$-strands 5 , 6 , and 7 in P92A resulted in a highly asymmetrical barrel wall with a 9.3 residue average length of strands 5 to 7 compared to an average length of 7.3 residues for strands 8 to 4 (Fig. 20C). The longer $\beta$-strands on taller side of the barrel wall likely also restrict the motions of the loops that are connected to these strands to a greater extent than the motions of the loops that are connected to the shorter side of the barrel wall. In marked contrast, the height of the barrel wall of wild-type OprG is much more uniform (Fig. 19 C), presumably leading to more similar mobilities of the residues in the four extracellular loops of the wild-type protein.

An inspection of the wild-type and P92A OprG structures also shows two girdles of aromatic residues positioned at the rims of the $\beta$-barrel with their side chains facing outwards toward the hydrophilic/hydrophobic interface of the lipid bilayer. The girdle on the periplasmic side is very well defined and consists of F6, F53, W57, Y98, F108, Y154, F162, and F207. The girdle on the extracellular side is more incomplete with only F13, Y168, and Y199 contributing. The presence of aromatic residues at the membrane interface is typical for many membrane proteins and particularly well pronounced in $\beta$ barrel OMPs. Aromatic side-chains anchor membrane proteins in the membrane and 
help to define the boundaries of the membrane-embedded portions of membrane proteins $[67,118]$.
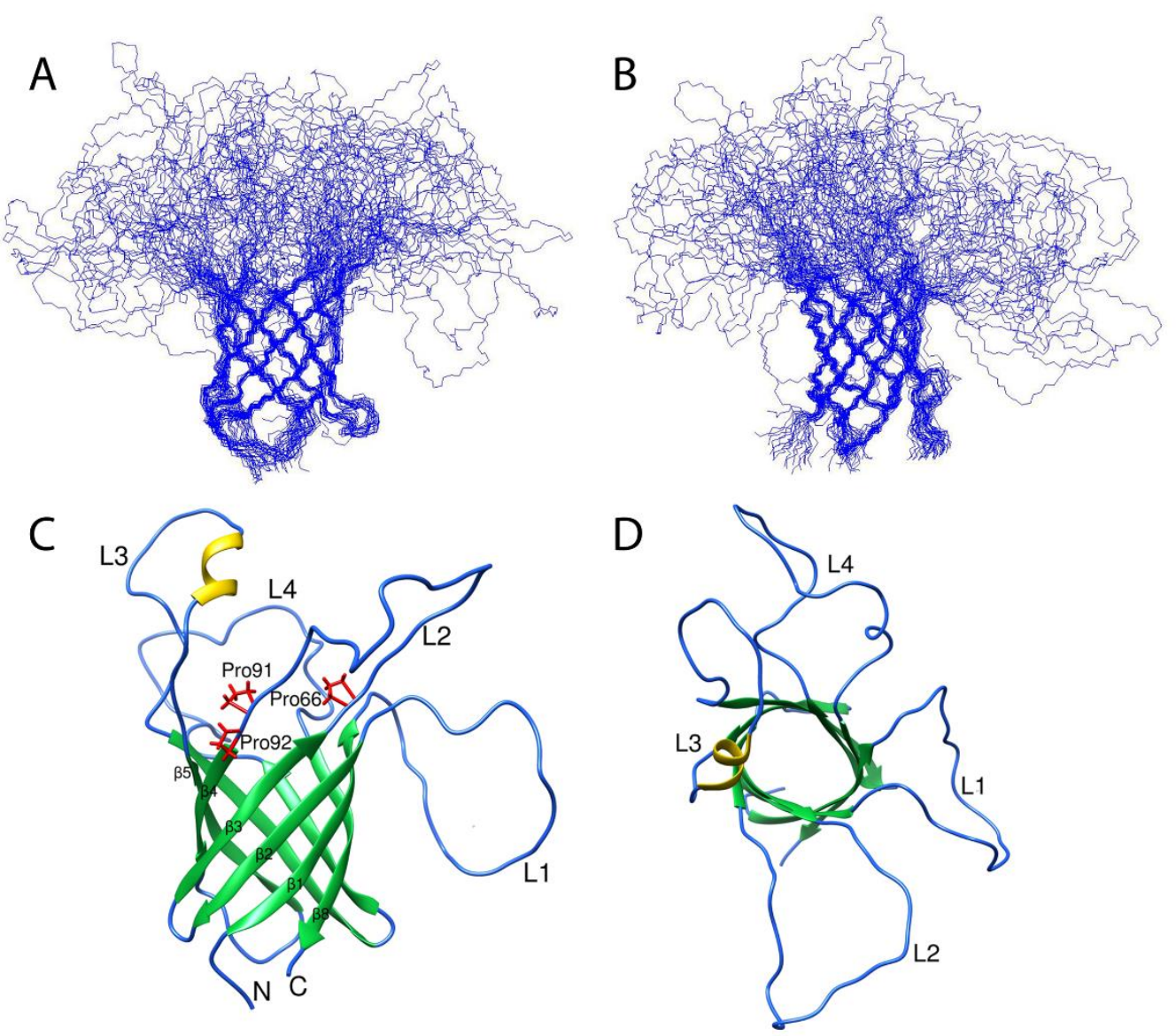

Figure 19. Solution structure of OprG in DHPC micelles. A and B: NMR ensemble of the 20 calculated lowest energy structures. Side view (C) and top-down view (D) of the lowest energy conformer of OprG from the ensemble of 20 lowest energy structures. The $\beta$ barrel, loop/turn and $\alpha$-helix regions are colored green, blue, and yellow, respectively, in $\mathbf{C}$ and $\mathbf{D}$. 

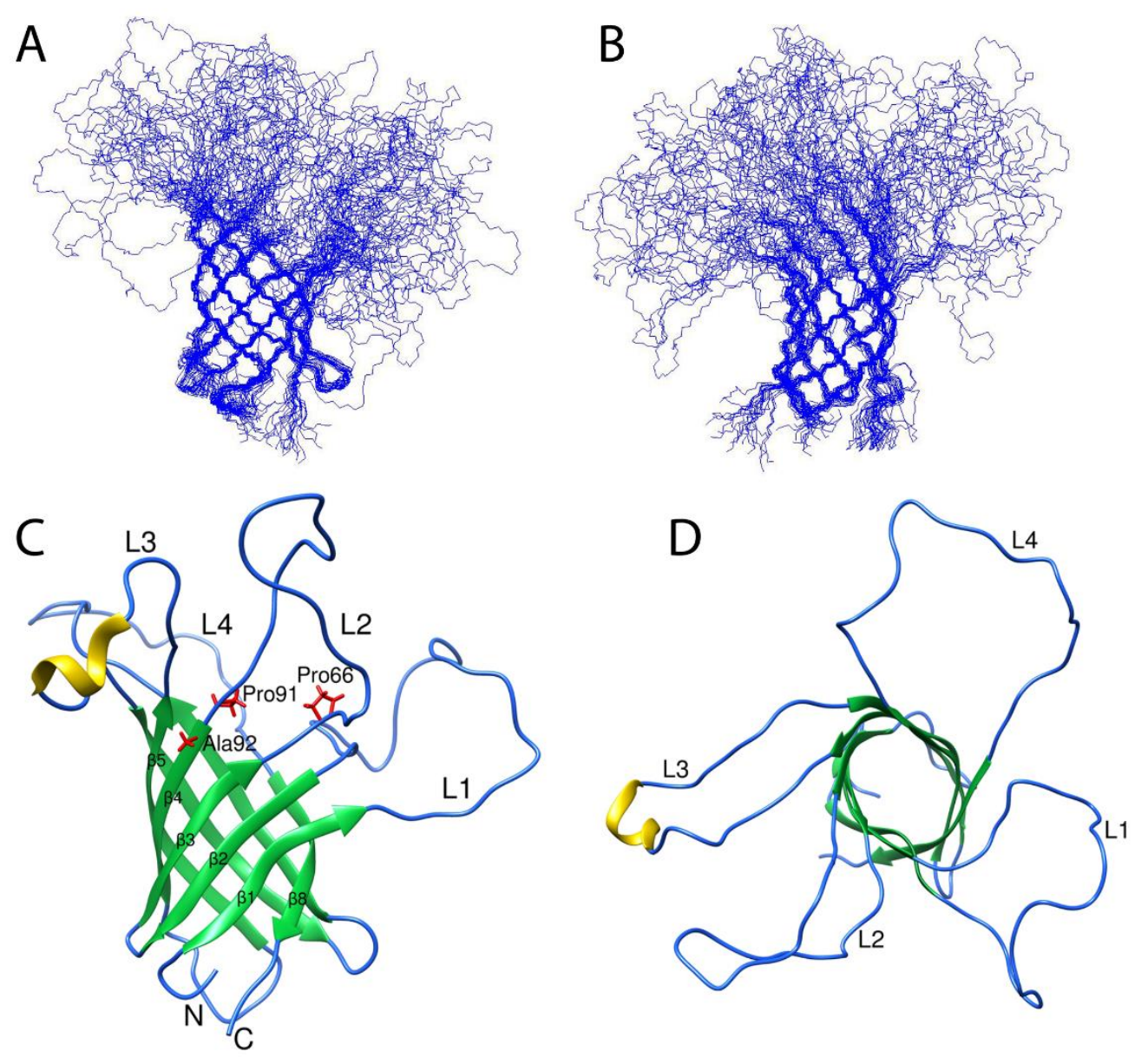

Figure 20. Solution structure of P92A OprG in DHPC micelles. A and B: NMR ensemble of the 20 calculated lowest energy structures. Side view (C) and top-down view (D) of the lowest energy conformer of P92A OprG from the ensemble of 20 lowest energy structures. The $\beta$-barrel, loop/turn and $\alpha$-helix regions are colored green, blue, and yellow, respectively, in $\mathbf{C}$ and $\mathbf{D}$. 
Table 3.

NMR and Refinement Statistics for wild-type and P92A OprG Structures in DHPC micelles ${ }^{\mathrm{a}}$

A. NMR distance and dihedral angle restraints

Structure Calculation

OprG P92A

Unique HN-HN NOE distances

Sequential 109

139

Medium range

Long Range

Intramolecular

Hydrogen bond restraints

0

Dihedral angle restraints

106

116

180

224

B. Violations

Distance constraints $(\AA)$

Dihedral angle restraints $\left(^{\circ}\right)$

Max. distance constraint $(>0.2 \AA)$

Max dihedral angle ( $>2.0 \AA$ )

Deviations from idealized geometry

Bond lengths $(\AA)$

Bond angles $\left({ }^{\circ}\right)$

Impropers

$$
\begin{gathered}
0.0043 \pm 0.0006 \\
0.0415 \pm 0.0093 \\
0 \\
0
\end{gathered}
$$

$0.00053 \pm 0.00005$

$0.253 \pm 0.00086$

$0.0872 \pm 0.0031$
$0.0081 \pm 0.001$

$0.0737 \pm 0.018$

0

0

$0.00061 \pm 0.00003$

$0.257 \pm 0.0022$

$0.0898 \pm 0.0066$

Ramachandran map analysis ${ }^{b}$

Most favored regions

$64.1 \%$

$65.7 \%$

Additionally allowed regions

$29.8 \%$

$29.1 \%$

Generously allowed regions

$4.0 \%$

$3.2 \%$

Disallowed regions

$2.1 \%$

$2.1 \%$

C. Ensemble RMSD

Mean global backbone r.m.s. deviation $(\AA \AA)$

B-sheet residues ${ }^{c}$

$0.98 \pm 0.21$

$0.89 \pm 0.18$

B-sheet and turn residues

$1.19 \pm 0.26$

$1.19 \pm 0.32$

All residues

Mean global heavy atom r.m.s. deviation ( $\AA$ )

$10.81 \pm 2.05$

$9.76 \pm 1.91$

B-sheet residues

$1.99 \pm 0.27$

$2.16 \pm 0.29$

B-sheet and turn residues

$2.13 \pm 0.31$

$2.30 \pm 0.32$

All residues

$11.13 \pm 1.96$

$10.05 \pm 1.77$

${ }^{a}$ Calculated from the 20 lowest-energy CNS conformers of each of the structures in DHPC micelles

${ }^{b}$ Calculated using PROCHECK-NMR

c $\beta$-sheet residues defined as 6-15,46-53,57-64,93-98,109-115,147-155,161-166,199-207 for wildtype OprG and 6-13,46-53,57-63,92-98,109-118,145-155,161-170,199-206 for P92A OprG from the mean of the 20 conformers. 
2.3.5 Dynamics of wild-type and P92A OprG in DHPC micelles. The overall rotational correlation times of the protein-micelle complexes were determined by onedimensional ${ }^{1} \mathrm{H}$-TRACT experiments [119]. They were 9 and 13 ns for wild-type and P92A OprG, respectively, when measured at $45^{\circ} \mathrm{C}$ in the $6.5-10.5 \mathrm{ppm}$ regions (Fig. $21 \mathrm{~A}$ and C). For comparison, the overall correlation times of two 8-stranded $\beta$-barrels of similar size, OprH and OmpX, were 22 and 21 ns, respectively [92, 119]. The low overall correlation times of both OprGs are probably the result of their relatively long disordered loops that tumble faster than the micelle-embedded barrel portions of both proteins. When only $\beta$-barrel peaks are included in the calculation (region 8.9-9.9 ppm), the overall rotational correlation times are 38 and 40 ns, for wild-type and P92A OprG, respectively (Fig. $\mathbf{2} \mathbf{1 B}$ and $\mathbf{D}$ ). The longer correlation times of the P92A mutant compared to the wild-type protein likely reflects the different lengths of $\beta$-strands 5,6 and 7 and loops 3 in the two proteins.

To examine the internal backbone dynamics on the ps to ns time scale, we measured the longitudinal $\left(T_{1}\right)$ and transverse $\left(T_{2}\right)$ relaxation times and the $\left({ }^{1} H\right)-{ }^{15} \mathrm{~N}$ NOEs of both proteins. As shown in Fig. 22 (top panels), the heteronuclear NOEs are largest for the residues in the $\beta$-barrel, indicating their restricted motions, and much smaller in the loops and turns, indicating increased flexibilities of these structural elements. Similarly, residues in the well-ordered barrel region have relatively long $T_{1}$ and short $T_{2}$ values in comparison with the much more flexible loop residues (Fig. 22, 
middle and bottom panels). The $\left({ }^{1} \mathrm{H}\right)-{ }^{15} \mathrm{~N} N O E, T_{1}$ and $T_{2}$ values generally follow the topology of wild-type and mutant OprG, confirming that the barrel portions are well structured and that the loops are largely disordered. Some significant differences between wild-type and P92A OprG are also evident. Loop 3 appears to be quite ordered in P92A, but highly flexible in wild-type OprG. The data show that loop 3 of wild-type undergoes large-scale motions on the ps to ns time scale, while it is rather rigid in P92A OprG. Although Pro92 is at the top of $\beta$-strand 4, it influences the flexibility of loop 3 that is attached to $\beta$-strands 5 and 6 , presumably through a modified hydrogen bonding pattern that surrounds Pro 92 in wild-type, but not in mutant OprG (Fig. 18 C and D).

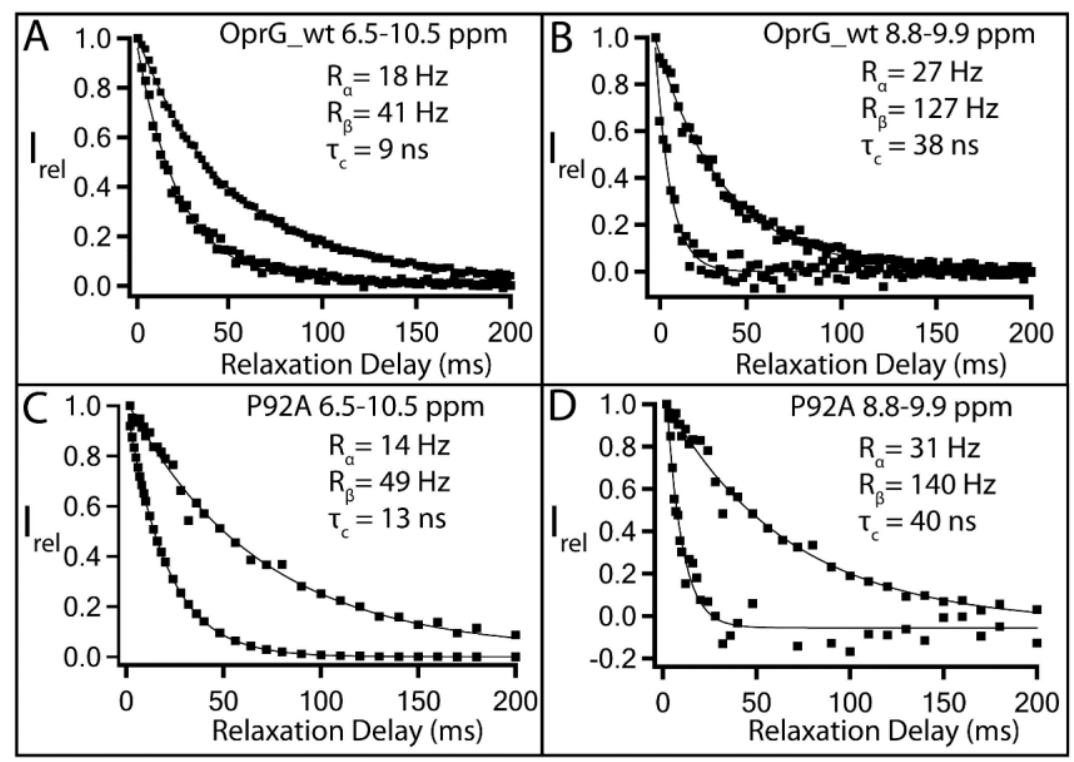

Figure 21. ${ }^{1} \mathrm{H}$ NMR signal decay curves of $\operatorname{OprG}(A, B)$ and P92A OprG (C, D) in DHPC micelles acquired by one-dimensional TRACT. The relative integrated signal intensities, Irel, from $6.5-10.5(\mathbf{A}, \mathbf{C})$ and 8.8-9.9 ppm of (B, D) each ${ }^{1} \mathrm{H}$ NMR spectrum are plotted versus relaxation delay times. The decay of the amide ${ }^{1} \mathrm{H}$ NMR signal is caused by the slow $(\alpha)$ and fast $(\beta)$ relaxing ${ }^{15} \mathrm{~N}$ spin-states. The respective relaxation rates were extracted by exponential curve fitting and the overall correlation times of 9 ns were determined for wild-type OprG and 13 ns for P92A OprG. The correlation time of $\beta$-barrel peaks region (8.8-9.9) was established to be $38 \mathrm{~ns}$ for OprG and $40 \mathrm{~ns}$ for P92A OprG. The data were collected at $800 \mathrm{MHz}$ and $45^{\circ} \mathrm{C}$. 


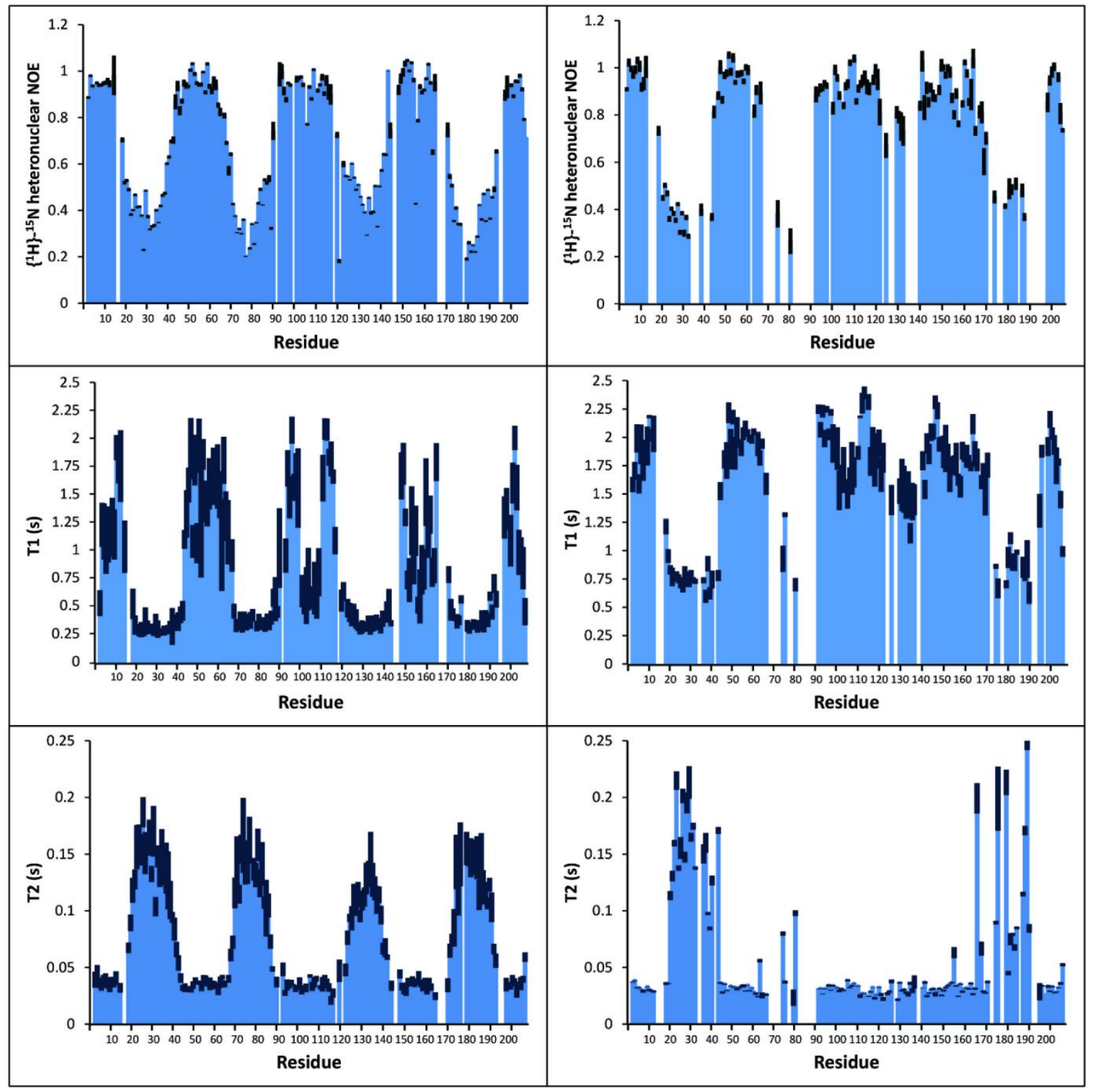

Figure 22. Backbone dynamics of OprG. $\left\{{ }^{1} \mathrm{H}-{ }^{15} \mathrm{~N}\right.$ heteronuclear NOEs (upper panels), longitudinal relaxation times (middle panels), and transverse relaxation times (lower panels) of OprG (left side) and P92A OprG (right side) in DHPC micelles determined at 800 $\mathrm{MHz}$ and $45^{\circ} \mathrm{C}$ are plotted as a function of the amino acid sequence. Black bars in the $\mathrm{T}_{1}$ and $\mathrm{T}_{2}$ plots are the upper limits of the standard deviations. Black bars in the NOE plots represent the upper limits of the standard errors. 


\subsection{Discussion}

The function of OprG in the OM of Pseudomonas aeruginosa has remained enigmatic since an early report on its potential physiological role as a low-affinity iron transporter [82]. Other previous studies suggested possible roles as diverse as a cation channel [73], a transporter of hydrophobic molecules via a lateral gating mechanism [74], or a facilitator of the inhibitory action of some antibiotics such as norflaxin, kanamycin, or tetracyclin on the growth of $P$. aeruginosa $[83,84]$. Like McPhee et al [73], we attempted to determine the electrophysiological activity of reconstituted OprG in black lipid membranes. Despite repeated efforts, we were unable to reproduce the large channel pores seen by these authors, but we did observe small broadly distributed OprG-related currents, which however were independent of any of the Pro mutations or any potential substrates or antibiotics that we added (data not shown). On the other hand, the combination of the liposome-swelling assay and cell growth experiments proved to be excellent for identifying new physiological substrates of OprG-mediated transport through the OM. These approaches indicated for the first time that OprG forms a pore for the permeation of small amino acids through lipid bilayers. Therefore, OmpW family proteins (Fig. 10) appear to have quite diverse functions with some homologs such as OmpW from P. fluorescens contributing to the uptake of polycyclic aromatic compounds [81], OprG from $P$. putida facilitating transport of hydrocarbons through the OM [107], and OprG from $P$. aeruginosa facilitating the uptake of small 
amino acids (this work). However, it should be kept in mind that the previous assignments of function in the other Pseudomonas species were not confirmed by protein purification and in vitro transport assays like we have done here for $\mathrm{P}$. aeruginosa OprG.

Our finding that OprG functions as a transporter for small amino acids may not be totally unexpected. Genes involved in a common metabolic pathway are usually organized into operons and even higher order organizations in bacterial genomes [120]. Therefore, possible functions of the oprG gene may be guessed by examining functions of neighboring genes. Although the exact functions of genes located next to oprG are presently unknown, BLAST searches of two neighboring gene loci of oprG in $P$. aeruginosa reveal that these genes may be involved in amino acid and peptide transport through the inner membrane of Gram-negative bacteria (Fig. 10 B). This interesting coincidence lends further indirect support that OprG may indeed be a facilitator of amino acid transport across the OM of $P$. aeruginosa.

How can this protein possibly facilitate the diffusion of small amino acids through the outer membrane? OprG forms a narrow channel, with both polar and apolar side chains facing the inside of the 8 -stranded $\beta$-barrel. The channel is probably too narrow to accommodate even the smallest amino acid, glycine that we found can be translocated by OprG. Alanine, serine, threonine, and valine would be even more difficult to fit into the small pore of OprG. A strong alternative possibility that has yet to 
be tested is that OprG may function as an oligomer in membranes and that the relevant amino acids are translocated along a yet to be defined interface between OprG protomers or OprG and the lipid bilayer. Our result that the P92A is unable to facilitate amino acid permeation through lipid bilayers in our liposome-swelling assay indicates that the unusual barrel wall in the region of the interfacial proline cluster likely contributes to the mechanism of amino acid transport by OprG.

The solution structure of wild-type OprG in DHPC micelles exhibits extracellular loops that are much more flexible than in the previously determined crystal structure [74]. The average length of $\beta$-strands in the crystal structure is 15.3 residues, extending well above the polar-apolar interface of the OM, while in solution it is 7.9 for wild-type and 8.6 for P92A OprG, i.e. about 24 to $26 \AA$, which approximately matches the hydrophobic thickness of the OM lipid bilayer [121]. Different lengths of well-structured $\beta$-barrels are commonly observed when crystal and solution structures of the same OMPs are compared. For example, the $\beta$-barrels of OmpA $[122,123]$, OmpG $[124,125]$, and OmpX $[126,127]$ are longer in crystals than in lipid micelles. The lengths of the $\beta$ barrels in the solution structures typically match the hydrophobic lengths of their outer perimeters ( $24 \AA)$ [128], whereas those of the crystal structures are often considerably longer with hydrophilic $\beta$-barrels extending into hydrophilic regions above the hydrophobic portions. The $\beta$-barrel in the NMR structural ensemble of OprG is also more circular in cross-section compared with the more elliptical $\beta$-barrel observed in the 
crystal structure. Apart from the different environments that may be responsible for this difference, it is also possible that the crystallization conditions favor just one of the multiple allowed conformations of the NMR structural ensemble. Regardless, it should be recognized that none of these structures were determined in their natural environments, i.e. in an asymmetric LPS-containing lipid bilayer. Crystal structures are determined in close-packed protein samples in which the extracellular loops often make multiple contacts to neighboring proteins, which could impart additional $\beta$-structure on them. Most NMR structures of OMPs, like the ones of OprG here, have been determined in lipid micelles, which are not physiological environments either. However, some were also determined in lipid nanodiscs [129], which provide more natural bilayer-like environments. Even in nanodiscs, the lengths of the $\beta$-barrels are still shorter than in the crystals. An interesting intermediate case is the $E$. coli protein OmpW, which showed concerted hinge motions of partially $\beta$-structured loops as measured by solution NMR in 30-Fos micelles [130]. We have recently made good progress with reconstituting OprG into different kinds of lipid nanodiscs that proved suitable for further NMR studies on this protein in a more bilayer-like and hence more physiological environment [131]. Although the spectra are not yet resolved well enough to allow for a structure determination of OprG in nanodiscs, it is already clear that the loops of OprG are also disordered in this more bilayer-like environment. 
Unlike in the crystal structure (Fig. 11 A), Pro61, Pro91, and Pro92 do not form a lateral gate and are not part of the $\beta$-barrel in the solution structures (Fig. 19 and 20). However, the barrel-to-loop transitions in the proline-rich region and elsewhere were differently affected by the proline-to-alanine mutation in the mutant compared to the wild-type solution structure. Therefore, and based on our activity measurements with many potential substrates, we consider it unlikely that hydrophobic substrates escape through a lateral gate into the hydrocarbon portion of the bilayer as has been proposed simply based on the crystal structure and in analogy with the fatty acid transporter FadL [74]. Rather than forming a lateral gate, the prolines contribute to the transitions of the barrel into loop 2 in both the wild-type and P92A structures (Fig. 19 and 20). However, the longer strands 4 and 5 in the mutant compared to the wild-type give rise to a highly asymmetric barrel rim in the mutant, but a more even and horizontal barrel rim in the wild-type protein. At the same time, loop 3 is much more ordered in P92A and more dynamic on the ps to ns time-scale in the wild-type protein (Fig. 20). Therefore, it seems likely that the symmetric barrel structure, the configuration of the Pro92 in the highly conserved proline cluster region, and/or the flexibility of loop 3 all contribute to the amino acid translocation activity of OprG. For comparison, it is interesting that loop flexibility of the Neisseria $\beta$-barrel protein Opa60 was also important for receptor recognition of this protein [132]. If OprG indeed forms a functional oligomer in the membrane, mutation of Pro92 could very well hamper or prevent oligomerization by direct Pro interaction, changes of the barrel shape, or altered loop dynamics. 
Alternatively or in addition, the observed dynamics of extracellular loops 2 and 3 may contribute to amino acid translocation by providing chaperoning environments in the membrane. Concerted loop motions have been shown to contribute to the function of another $\beta$-barrel OM protein [133].

Unfortunately the P66A, P91A, and double Pro mutants did not stably insert into lipid bilayers to assess the effects of these potentially interesting mutations on the amino acid translocation activity of OprG. These proteins also gave NMR spectra of much poorer quality, which prevented us from determining changes in their dynamic structures, which might have offered additional clues on the mechanism of amino acid transport by OprG. Despite these limitations, these experiments informed us that the proline cluster region is critically important for obtaining stable and functional proteins in micelles and lipid bilayers. It will therefore be interesting to further examine the critical role that this region plays in the mechanism of small amino acid transport across the OM of $P$. aeruginosa.

In conclusion, the results of this work provide a structural and dynamic framework to formulate new hypotheses on how OprG-facilitated amino acid transport across the OM might work. It will be interesting to test and distinguish between such hypotheses in future work and it will be particularly interesting to see if any of the proposed mechanisms might affect the antibiotic resistance of Pseudomonas aeruginosa and its cytotoxic effects on epithelial cells in the lungs of infected patients. 


\section{Chapter 3: Molecular Interactions of}

\section{Lipopolysaccharide with an Outer Membrane}

\section{Protein from Pseudomonas aeruginosa Probed by}

\section{Solution NMR}

Iga Kucharska, Binyong Liang, Nicholas Ursini, and Lukas K. Tamm

\section{1 Introduction}

Pseudomonas aeruginosa is an opportunistic human pathogen and the most common cause of lung infections in cystic fibrosis patients [102, 103]. $P$. aeruginosa is also responsible for high numbers of infections in hospital environments, including urinary tract, wound and skin infections $[1,4]$. Compared with other pathogens, $P$. aeruginosa infections are very challenging to treat as this bacterium displays high intrinsic resistance to a wide range of antibiotics, including fluoroquinolones, aminoglycosides, and $\beta$-lactams. This is mainly caused by the high stability and low permeability of its outer membrane (OM), which is estimated to be 12 to 100 times less permeable than that of $E$. coli, as well as the ability of $P$. aeruginosa to form resistant biofilms [7, 8]. Lipopolysaccharides (LPS), which make up the outer leaflet of the OM, contribute a great deal to the high stability of $P$. aeruginosa's OM. LPS produced by $P$. 

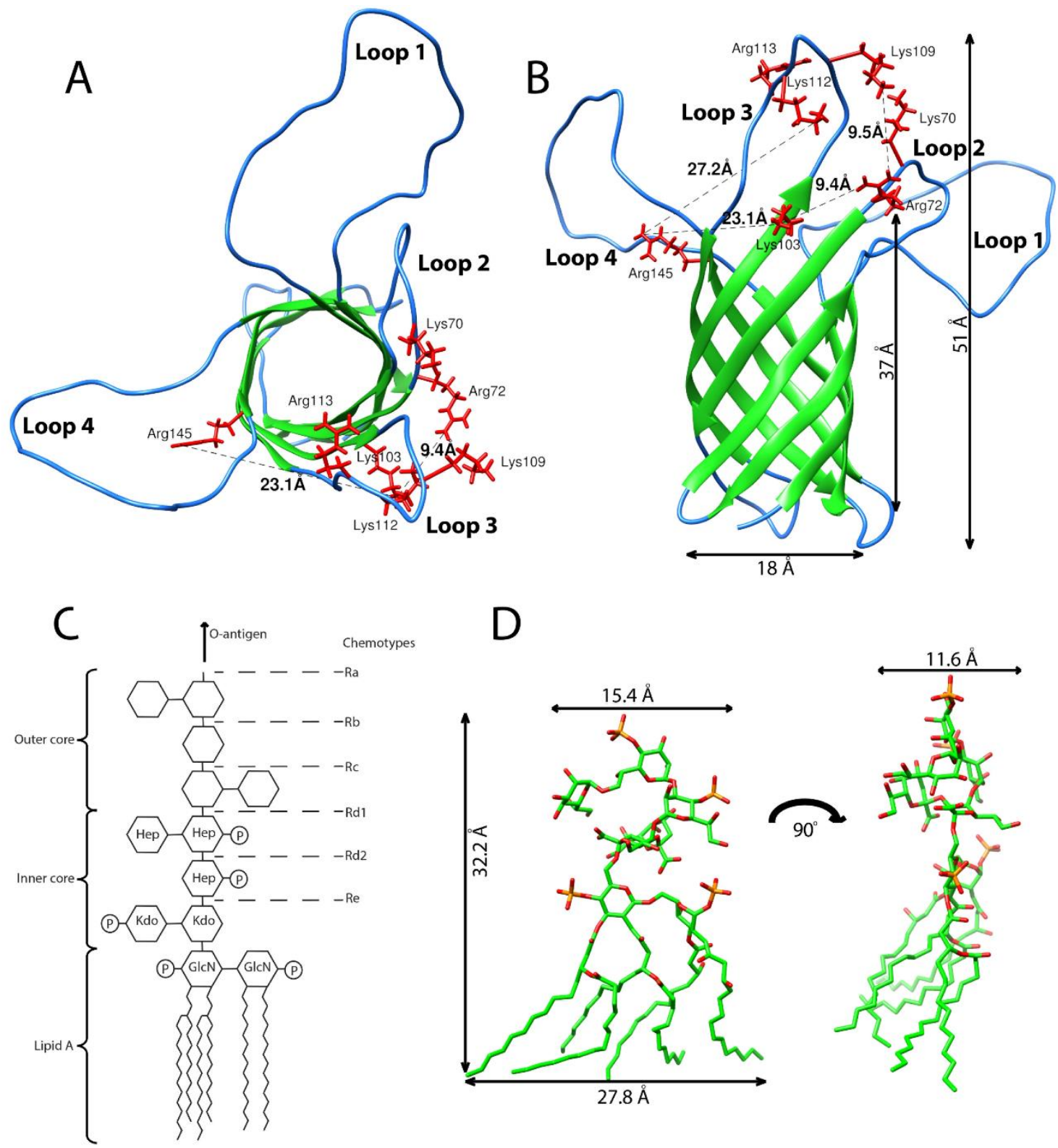

$\mathrm{D}$

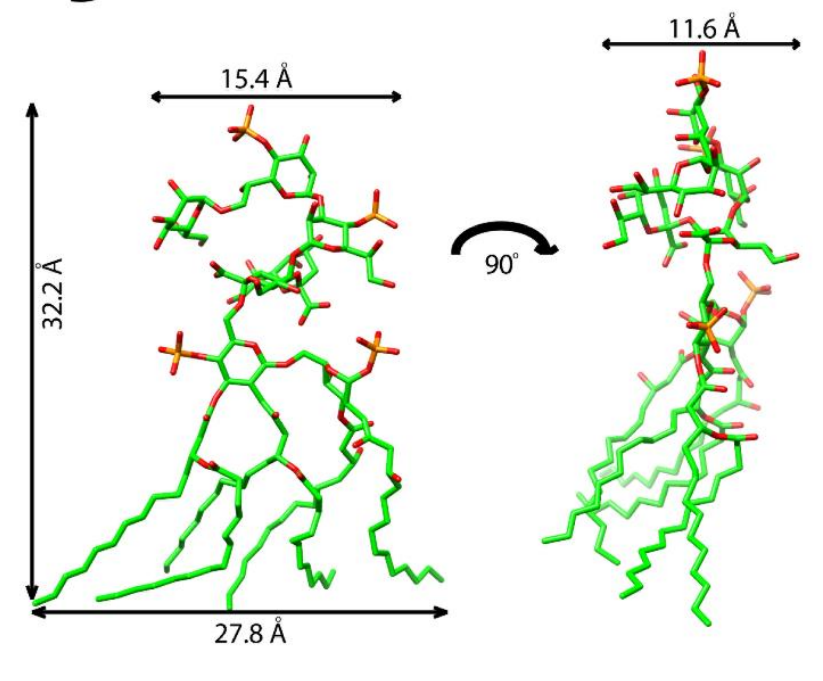

Figure 23. Comparison of structures of OprH and LPS. (A) and (B) OprH from $P$. aeruginosa (PDB code: $2 \mathrm{LHF}$ ). Charged side chains that might interact with LPS are highlighted in red and the distances between the distal residues are shown as black dashed lines. (C) Schematic chemical structure of E. coli LPS. LPS is composed of lipid A, inner and outer core oligosaccharides, and O-antigens. GlcN, D-glucosamine; Kdo, 3-deoxy-D-manno-octulsonic acid; Hep, L-glycero-D-mannoheptose; P, phosphate. (D) Dimension of Rd LPS based on the crystal structure of the TLR4/MD-2/Ra LPS complex (PDB code: 3FXI). 
aeruginosa is a main factor in the virulence as well as innate and acquired host responses to infection [13]. LPS has a complex structure (Fig. 23). Its hydrophobic portion is lipid A, which by itself can elicit cytotoxicity. Attached to lipid A is a hydrophilic core polysaccharide chain, followed by the hydrophilic O-antigenic polysaccharide chain [14]. Removal of divalent cations from LPS by chelating agents and the binding of polycationic antibiotics such as polymyxins and aminoglycosides to LPS may lead to a destabilization and rupture of the OM [134].

OprH is a $21-\mathrm{kDa}$ protein integral to the OM of $P$. aeruginosa. OprH is genetically linked to the PhoP-PhoQ two-component regulatory system that is up-regulated in response to $\mathrm{Mg}^{2+}-$ limited growth conditions [8, 93]. The PhoP-PhoQ system is also involved in virulence and in resistance to cationic antimicrobial peptides and polymyxin [93]. OprH is further up-regulated in bacteria that adhere to human bronchial epithelial cells 5,000-fold compared to non-adherent bacterial cells [97]. This up regulation occurs even when the culture medium contains millimolar concentrations of $\mathrm{Mg}^{2+}$ that would normally suppress transcription of the oprH-phoP-phoQ operon [96]. OprH might be a promising alternative target for antimicrobial treatments, as there has been a growing number of $P$. aeruginosa strains that are able to chemically modify their lipid A structure to gain resistance to last resort antibiotics like polymyxins [135-137].

Solution NMR in 1,2-dihexanoyl-sn-glycero-3-phosphocholine (DHPC) micelles was used to determine the eight-stranded $\beta$-barrel structure of $\mathrm{OprH}$, which also 
features four extracellular loops of unequal size (Fig. 23). NMR relaxation experiments revealed that the extracellular loops are unstructured and highly dynamic on the ps to ns time-scale [92]. As mentioned, OprH is up-regulated and overexpressed so that it becomes a major component of the $\mathrm{OM}$ when $P$. aeruginosa is grown under limiting concentrations of divalent cations. Therefore, $\mathrm{OprH}$ is believed to act as a surrogate for $\mathrm{Mg}^{2+}$ and $\mathrm{Ca}^{2+}$ by cross-linking LPS, thereby tightening the OM during divalent cation deficiency [93]. The direct interaction of LPS with OprH was demonstrated using several in vitro and in vivo techniques, including NMR chemical shift perturbation, trypsin protection, and pull-down experiments.

Based on NMR chemical shift perturbations observed upon the addition of LPS to OprH in lipid micelles, it was concluded that the interaction is predominantly electrostatic and localized to charged regions near the extracellular rim of the barrel and loops 2 and 3 of OprH [92]. To characterize the binding site of LPS on OprH in more detail, we conducted a more comprehensive NMR study measuring chemical shift perturbations of backbone and side chain resonances upon the addition of LPS to OprH. We also removed several charged residues individually and in combination to test their effects on LPS binding. Combining our NMR results with binding studies using an enzyme-linked immunosorbent assay (ELISA) allowed us to present a more definitive molecular model for the interactions between LPS and OprH and offer new insight into protein-lipid interactions that may contribute to the antibiotic resistance during $P$. aeruginosa infections. 


\subsection{Methods}

3.2.1 Expression, Purification and Refolding of OprH and OprG. OprH from $P$. aeruginosa strain PAO1 and all OprH mutants were expressed in BL21 (DE3) E. coli cells as described in $[92,131]$. All OprH constructs used were without the $\mathrm{N}$-terminal signal sequence (residues 1-22 were replaced with Met-1 so that Ala-23 becomes Ala-2 in our numbering system) and either with a C-terminal His 6 -tag or Strep-tag II (SAWSHPQFEK). All proteins expressed into inclusion bodies in high yields.

${ }^{2} \mathrm{H}-{ }^{13} \mathrm{C}-,{ }^{15} \mathrm{~N}$-labeled OprH with loops 1 and 4 deleted $(\mathrm{OprH} \Delta \mathrm{L} 1 \Delta \mathrm{L} 4)$ was used for the sequential assignment of the backbone and ${ }^{13} \mathrm{C}-,{ }^{15} \mathrm{~N}$-labeled $\mathrm{OprH} \Delta \mathrm{L} 1 \Delta \mathrm{L} 4$ was utilized for the assignment of side chains. For the preparation of $\left[\mathrm{U}-{ }^{2} \mathrm{H},{ }^{15} \mathrm{~N}\right]$; $\|$ le $\delta 1-$ $\left[{ }^{13} \mathrm{CH}_{3}\right]$; Leu,Val-[ $\left[{ }^{13} \mathrm{CH}_{3},{ }^{12} \mathrm{CD}_{3}\right]$-labeled samples, OprH $\Delta \mathrm{L} 1 \Delta \mathrm{L} 4$ cells were first grown overnight at $37^{\circ} \mathrm{C}$ in $20 \mathrm{ml}$ of unlabeled minimal media, then centrifuged at 5,000 rpm for 10 min, washed with $5 \mathrm{ml}\left[\mathrm{U}^{2} \mathrm{H}^{15} \mathrm{~N}\right]$ minimal medium, centrifuged again, resuspended in $1 \mathrm{~L}$ of ${ }^{15} \mathrm{~N}$ minimal medium, and grown at $37^{\circ} \mathrm{C}$. When the $0 D 600$ reached $0.4,60 \mathrm{mg}$ of 2-keto-3- $d 2-4-{ }^{13} \mathrm{C}$-butyrate and $100 \mathrm{mg}$ of 2-keto-3-methyl-d3-3d1-4- ${ }^{13}$ C-butyrate (Cambridge Isotope Laboratories) were added [138]. After one hour the temperature was lowered to $25^{\circ} \mathrm{C}$ and protein overexpression was induced with 1 $\mathrm{mM}$ isopropyl- $\beta$-D-thiogalactopyranoside for $\sim 16$ hours. 
$\mathrm{His}_{6}$-tagged proteins were purified from inclusion bodies and refolded following the published protocol $[131,139]$. Protein samples with a Strep-tag II used for NMR studies were solubilized from inclusion bodies in denaturation buffer ( $8 \mathrm{M}$ urea, $10 \mathrm{mM}$ MES pH 6.0, 0.1 mM EDTA) and loaded onto a hiTrap CM sepharose FF column (GE Healthcare Life Sciences). Proteins were eluted with a $0-250 \mathrm{mM} \mathrm{NaCl}$ gradient in denaturation buffer, concentrated to $\sim 1 \mathrm{ml}$ and subsequently refolded. We also tried to use Strep-Tactin sepharose (iba) in our purification protocol, but we obtained only little to no protein binding to this resin. The final NMR samples were concentrated to $0.2-$

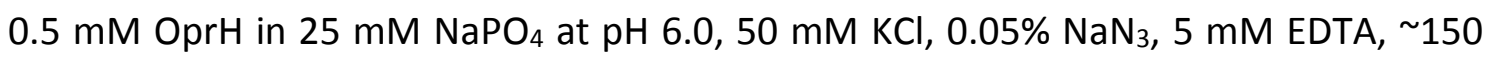
$\mathrm{mM}$ DHPC and $5 \% \mathrm{D}_{2} \mathrm{O}$.

Proteins with a Strep-tag II for ELISA were refolded immediately after solubilizing in inclusion bodies, then purified by gel-filtration on a Superdex 200 column (GE Healthcare Life Sciences) equilibrated with 20 mM HEPES pH 7.3, 150 mM NaCl, 1 mM EDTA, $0.1 \%$ DPC. Proteins at concentrations of $\sim 0.5 \mathrm{mg} / \mathrm{ml}$ with good purity were obtained using this procedure, which effectively removed all free arginine used in the refolding buffer. The proteins were quantitatively refolded as seen from the shift of their apparent molecular masses on SDS-PAGE gels.

OprG from P. aeruginosa strain PAO1 was expressed in BL21 (DE3) E. coli cells as described in $[131,139]$. The OprG construct used was without the $\mathrm{N}$-terminal signal sequence (residues 1 to 26 were replaced with Met 1 so that His27 becomes His2 in our 
numbering system) and with a C-terminal Strep-tag II (SAWSHPQFEK). OprG-Strep-Tag II was purified as OprH-Strep-Tag II for ELISA. SDS-PAGE gels showed pure and quantitatively refolded proteins.

3.2.2 Site-directed mutagenesis. The $\operatorname{OprH} \Delta \mathrm{L} 1 \Delta \mathrm{L} 4$ mutant with loops 1 and 4 partially deleted was constructed by removing residues $17-38$ and 150-167. Primers were designed to mutate residues $70,72,103,113$ and 145 to glutamines and to generate the double mutant $\mathrm{R} 72 \mathrm{Q} / \mathrm{K} 103 \mathrm{Q}$ and the triple mutant $\mathrm{K} 70 \mathrm{Q} / \mathrm{R} 72 \mathrm{Q} / \mathrm{K} 103 \mathrm{Q}$. The Stratagene QuikChange site-directed mutagenesis kit was used to make all mutations starting with wild-type OprH.

3.2.3 Mass Spectroscopy. Samples of Rd2 LPS from E. coli F583 were prepared and analyzed by matrix-assisted laser desorption ionization-time of flight (MALDI-TOF) mass spectrometery as described in [140]. A small amount of the LPS was solubilized in a mixture of methanol/water (1:1) containing $5 \mathrm{mM}$ EDTA and dissolved by brief ultrasonication. A few microliters of the obtained mixture were then desalted on a small piece of Parafilm with some grains of cation-exchange beads (Dowex 50WX8-200, Sigma), previously converted into the ammonium form. $0.3 \mu \mathrm{l}$ of this sample solution was deposited, together with the same volume of $20 \mathrm{mM}$ dibasic ammonium citrate, in a thin layer of homogeneous matrix film obtained from a solution, the components of which were 2,4,6-trihydroxyacetophenone (THAP), $200 \mathrm{mg} / \mathrm{ml}$ in methanol, and 15 
$\mathrm{mg} / \mathrm{ml}$ nitrocellulose (Trans-blot membrane, BioRad) in acetone/propan-2-ol (1:1 v/v), mixed in a 4:1 v/v ratio.

\subsubsection{Visualization of LPS on SDS-PAGE gels by zinc-imidazole staining. Rd2 LPS}

E. coli F583 was visualized on SDS-PAGE gels using zinc-imidazole staining, as described in [141]. Briefly, after electrophoresis, the gel was incubated in $400 \mathrm{ml}$ boiling water (3 times for $15 \mathrm{~min}$ ) to remove electrophoresis reagents. The water was removed and the gel was incubated in $10 \mathrm{mM}$ zinc sulfate for $15 \mathrm{~min}$. To develop the image, the gel was soaked with agitation in $0.2 \mathrm{M}$ imidazole for 1 to 3 min until a homogeneous white background developed on the gel surface except in the zones of the LPS bands, which remained transparent. After sufficient image contrast was attained, the gel was rinsed three times with 50 to $100 \mathrm{ml}$ distilled water to remove excess reagent. The appearance of the negative staining pattern was observed by placing the gel over any dark surface.

3.2.5 NMR Spectroscopy. All NMR experiments were recorded at $45^{\circ} \mathrm{C}$ on a Bruker Avance III 800 spectrometer equipped with a triple-resonance cryoprobe. All double- and triple-resonance experiments were performed using the Bruker Topspin version 2.1.6 software suite. Sequential backbone assignments of $\mathrm{OprH} \Delta \mathrm{L} 1 \Delta \mathrm{L} 4$ were obtained by recording TROSY versions of HNCA, HNCB and HNCO experiments. Assignments of the ${ }^{1} \mathrm{H}-{ }^{13} \mathrm{C}$ HMQC spectrum of $\left[\mathrm{U}-{ }^{2} \mathrm{H},{ }^{15} \mathrm{~N}\right]$; $\|$ le $\delta 1-\left[{ }^{13} \mathrm{CH}_{3}\right]$; Leu,Val- $\left[{ }^{13} \mathrm{CH}_{3},{ }^{12} \mathrm{CD}_{3}\right]$ OprH $\Delta \mathrm{L} 1 \Delta \mathrm{L} 4$ were obtained by first collecting and assigning $\mathrm{H}(\mathrm{CCO}) \mathrm{NH}-\mathrm{TOCSY}$ and $(\mathrm{H}) \mathrm{C}(\mathrm{CO}) \mathrm{NH}-\mathrm{TOCSY}$ spectra. All triple-resonance experiments 
were recorded in a non-uniformly sampled manner [142] using Poisson-gap sampling schedules [143] and reconstructed using the iterative soft threshold method [144]. All NMR data were processed with NMRPipe and analyzed with Sparky software [111].

3.2.6 Enzyme-Linked Immunosorbent Assay. ELISAs were performed following to the protocol of [145], with several modifications. Nunc MaxiSorp ELISA plates (BioLegend) were coated by incubating $100 \mu \mathrm{l}$ of $30 \mu \mathrm{g} / \mathrm{ml}$ LPS from E. coli F583 (Sigma) in $0.1 \mathrm{M} \mathrm{Na}_{2} \mathrm{CO}_{3}, 20 \mathrm{mM}$ EDTA, pH 9.6, overnight at $37^{\circ} \mathrm{C}$. The LPS solution was flicked out, and the plates were rinsed three times with water. Excess binding sites were blocked with $100 \mu \mathrm{l} /$ well of $10 \mathrm{mg} / \mathrm{ml}$ BSA in $50 \mathrm{mM}$ HEPES, $0.15 \mathrm{M} \mathrm{NaCl}, 1 \mathrm{mM}$ EDTA, $\mathrm{pH} 7.4$ for $30 \mathrm{~min}$ at $37^{\circ} \mathrm{C}$. Refolded protein samples with the C-terminal Strep-tag II were added in a total volume of $100 \mu \mathrm{l} /$ well diluted in $50 \mathrm{mM} \mathrm{HEPES}, 0.15 \mathrm{M} \mathrm{NaCl}, 1$ mM EDTA, $1 \mathrm{mg} / \mathrm{ml}$ BSA, 0.05\% Tween 20 (Sigma) (buffer A). Binding was allowed to occur for $3 \mathrm{~h}$ at $37^{\circ} \mathrm{C}$. The plate was then rinsed three times with $200 \mu \mathrm{l} /$ well of buffer A. Anti-Strep II StrepMAB-Classic antibody (iba), $100 \mu \mathrm{l} /$ well diluted $5000 \mathrm{x}$ in buffer A was added to each well and incubated for $1 \mathrm{~h}$ at room temperature with agitation. Subsequently the plate was rinsed three times with $200 \mu \mathrm{l} /$ well of buffer A. Anti-mouse IgG peroxidase conjugate antibody (Sigma), $100 \mu \mathrm{l} /$ well diluted $5000 \mathrm{x}$ in buffer A was added and incubated in the wells for $1 \mathrm{~h}$ at room temperature with agitation followed by three washes of $200 \mu \mathrm{l} /$ well buffer $A$. The peroxidase substrate $3,3^{\prime}, 5,5^{\prime}$ tetramethylbenzidine (Sigma) $100 \mu \mathrm{l} /$ well was added undiluted to each well and incubated for $15 \mathrm{~min}$ at room temperature with agitation. The reaction was stopped by 
addition of $0.5 \mathrm{M}$ sulfuric acid and the absorbance was read at $450 \mathrm{~nm}$ on a SpectraMax M5 (Molecular Devices) plate reader. The mean absorbances and standard errors were calculated from six replicates of each well.

3.2.7 Lysine methylation. Reductive methylation was performed following the protocols of $[146,147]$ with the following modifications. Briefly, $20 \mu$ of a $1 \mathrm{M}$ boraneammonia complex $\left(\mathrm{NH}_{3}-\mathrm{BH}_{3}\right)$ and $40 \mu \mathrm{l}$ of $1 \mathrm{M}$ formaldehyde were added to $1 \mathrm{ml}$ of 0.5 M purified and unfolded OprH in $50 \mathrm{mM} \mathrm{NaPO}_{4}$ at $\mathrm{pH} 8.0,300 \mathrm{mM} \mathrm{NaCl}, 8 \mathrm{M}$ urea, 250 $\mathrm{mM}$ imidazole, and the reaction was incubated for $2 \mathrm{~h}$ with stirring at $25^{\circ} \mathrm{C}$. The addition of the borane-ammonia complex and formaldehyde was repeated, and the mixture was incubated for another $2 \mathrm{~h}$. After the addition of another $10 \mu \mathrm{l}$ of $1 \mathrm{M}$ borane-ammonia complex, the reaction mixture was incubated at $25^{\circ} \mathrm{C}$ with stirring overnight. The reaction was stopped by adding glycine to $200 \mathrm{mM}$, and undesired reaction products as well as excess reagents were removed by purification on PD-10 desalting column (GE Healthcare Life Sciences). The resulting lysine-methylated OprH was refolded using the same procedure as unmodified, wild-type OprH. After refolding, the labeling was confirmed by MALDI analysis.

\subsubsection{Determination of LPS-OprH dissociation constants by NMR. The} dissociation constant for the binding of LPS to OprH was determined by monitoring the change in chemical shifts of ${ }^{15} \mathrm{~N}-{ }^{1} \mathrm{H}$-TROSY cross-peaks as a function of the LPS concentration as described in [148]. 0.1 to $0.15 \mathrm{mM}{ }^{15} \mathrm{~N}$-labeled OprH, OprH-R72Q or 
OprH $\Delta \mathrm{L} 1 \Delta \mathrm{L} 4$ in $25 \mathrm{mM} \mathrm{NaPO}_{4}$ at pH 6.0, $50 \mathrm{mM} \mathrm{KCl}, 0.05 \% \mathrm{NaN}_{3}, 5 \mathrm{mM}$ EDTA, 150 mM DHPC and $5 \% \mathrm{D}_{2} \mathrm{O}$ was titrated with seven or eight additions of $\mathrm{Rd} 2$ LPS from E. coli F583 so that the final concentration of LPS was 1.7-4.0 mM. ${ }^{15} \mathrm{~N}$ TROSY spectra were recorded in triplicate with each successive addition. The difference in chemical shift of an atom with no LPS present and that in the presence of LPS $\left(\Delta \delta_{\text {obs }}\right)$ was plotted against the total amount of LPS added (LPS tot) and fitted with the equation:

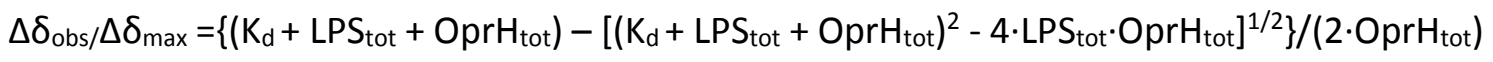
(Eq. 1)

where $\mathrm{K}_{\mathrm{d}}$ is the dissociation constant, $\mathrm{OprH}_{\text {tot }}$ is the total amount of OprH present in solution and $\Delta \delta_{\max }$ is the maximum chemical shift difference seen for the given residue of the protein saturated with LPS.

\subsection{Results}

\subsubsection{Characterization of different species of LPS used for assessing their}

interactions with OprH. Different species and strains of Gram-negative bacteria produce different varieties of LPS. In order to analyze interactions of LPS with OprH by NMR, we tested multiple products of LPS for their suitability in interaction studies by solution NMR. Commercially available smooth LPS from P. aeruginosa (Sigma, \#L8643, \#L9143, \#L7018) has highly variable chemical compositions and contents of impurities. 

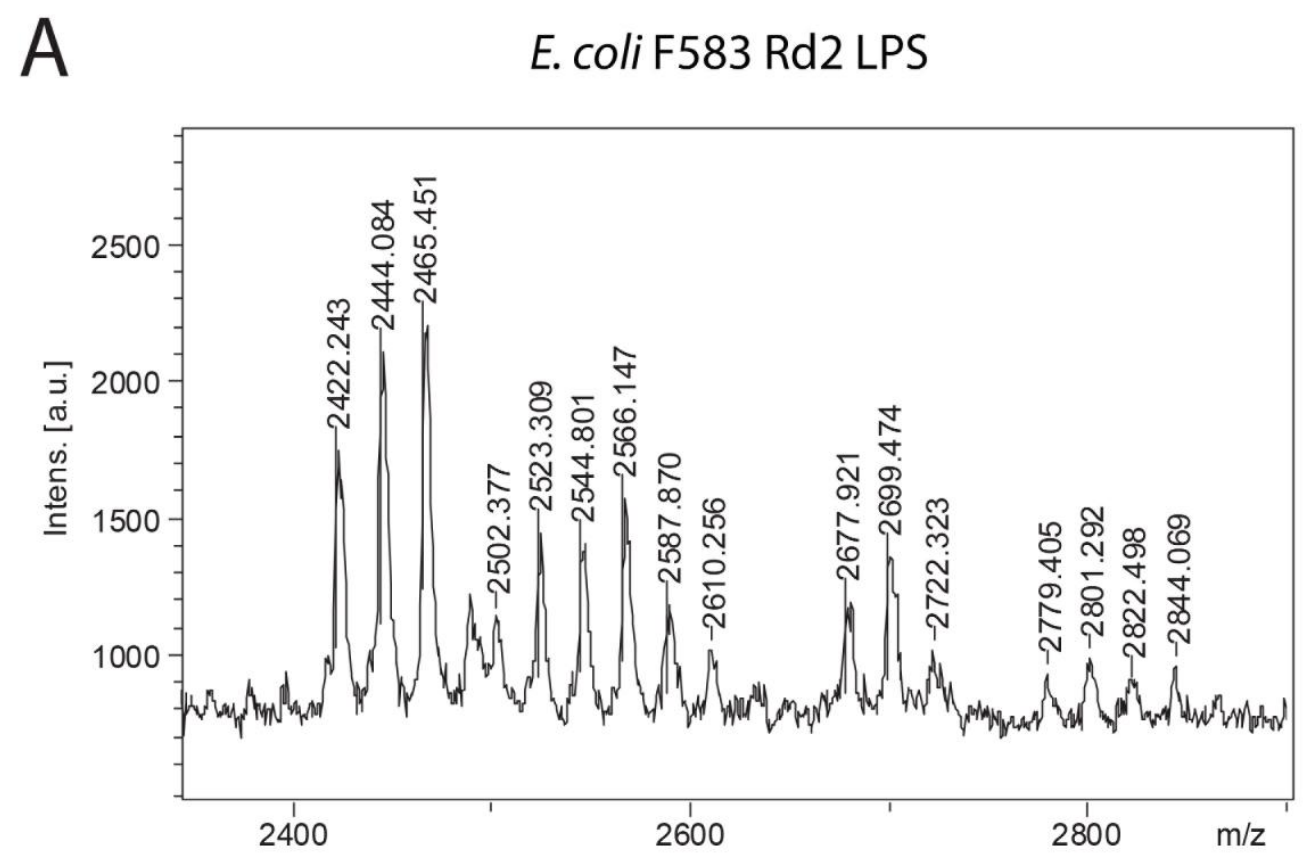

B $\quad k D a$

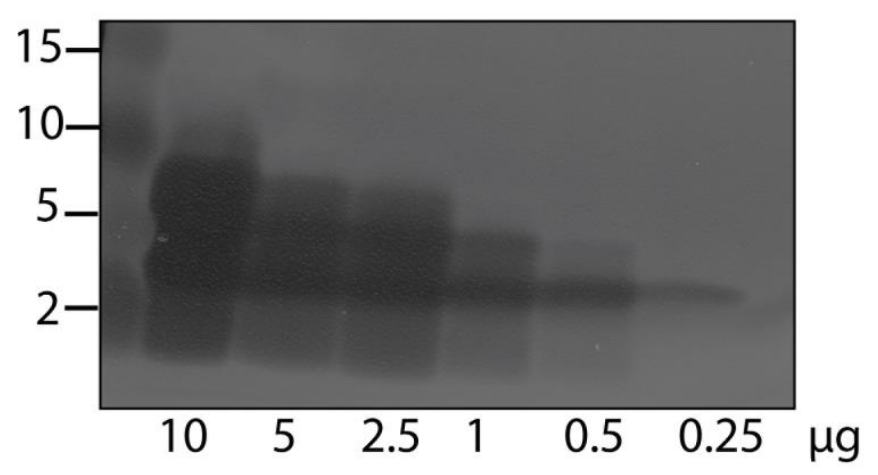

Figure 24. Characterization of Rd2 LPS from E. coli F583. (A) MALDI mass spectrum of $\mathrm{Rd} 2 \mathrm{LPS}$ from $E$. coli $\mathrm{F} 583$ with $\mathrm{m} / \mathrm{z}$ values of the main peaks indicated. (B) Zinc-imidazolestained SDS-PAGE gel with different amounts of Rd2 LPS from E. coli F583 applied to lanes as indicated.

We found that when used at high concentrations needed for NMR, these sources of LPS produce viscous solutions that deteriorate the quality of NMR spectra. Deep rough (Rd2) LPS from E. coli strain F583 (Sigma, \#L6893) (Fig. 23 C) behaved better and was used in 
studies that required a high excess of LPS over protein concentrations, most notably the NMR titration studies. To verify the molecular mass of Rd2 LPS from E. coli strain F583 we performed MALDI-MS analysis (Fig. 24 A) and SDS-PAGE electrophoresis followed by staining with zinc and imidazole salts [141] (Fig. 24 B). Both techniques confirmed that the molecular mass of $\mathrm{Rd} 2$ LPS was $\sim 2.7 \mathrm{kDa}$. Best results for NMR assignments and chemical shifts of OprH were obtained with Kdo2-lipid A (Avanti Polar Lipids, \#699500P), which lacks the outer core polysaccharide rings. The chemical structure (Fig. 23 C and D) and molecular mass (2306 Da) of this product is better defined and more uniform compared to other commercially available LPS samples. We confirmed that the ${ }^{15} \mathrm{~N}$ TROSY spectrum of OprH with Kdo2-lipid A was similar, but of better quality than the previously reported spectrum of OprH in the presence of $P$. aeruginosa smooth LPS [92]. The solubility of Kdo2-lipid A is lower than that of Rd2 LPS, but is still sufficient for most of the NMR work reported in this study.

\subsubsection{NMR Chemical Shift Perturbation of $\Delta \mathrm{L1}, \Delta \mathrm{L} 4$ Mutant of OprH by Kdo2-}

Lipid A. We previously showed that the interaction of OprH with LPS is mainly mediated through loops 2 and 3 [92]. Therefore and to simplify assignments, we used an OprH construct with loops 1 and 4 deleted $(\mathrm{OprH} \Delta \mathrm{L} 1 \Delta \mathrm{L} 4)$ in this work. This protein yielded a ${ }^{15} \mathrm{~N}-{ }^{1} \mathrm{H}-$ TROSY spectrum with significantly less overlap in the region from 7.8 to $8.6{ }^{1} \mathrm{H}$ ppm compared to wild-type OprH, which permitted a de novo assignment of this construct (Fig. 25 A). To determine which specific residues of OprH $\Delta \mathrm{L} 1 \Delta \mathrm{L} 4$ interact with LPS, Kdo2-lipid A was added to $0.2 \mathrm{mM}{ }^{2} \mathrm{H}-,{ }^{13} \mathrm{C}-,{ }^{15} \mathrm{~N}$-labeled $\mathrm{OprH} \Delta \mathrm{L} 1 \Delta \mathrm{L} 4$ in $\mathrm{DHPC}$ 


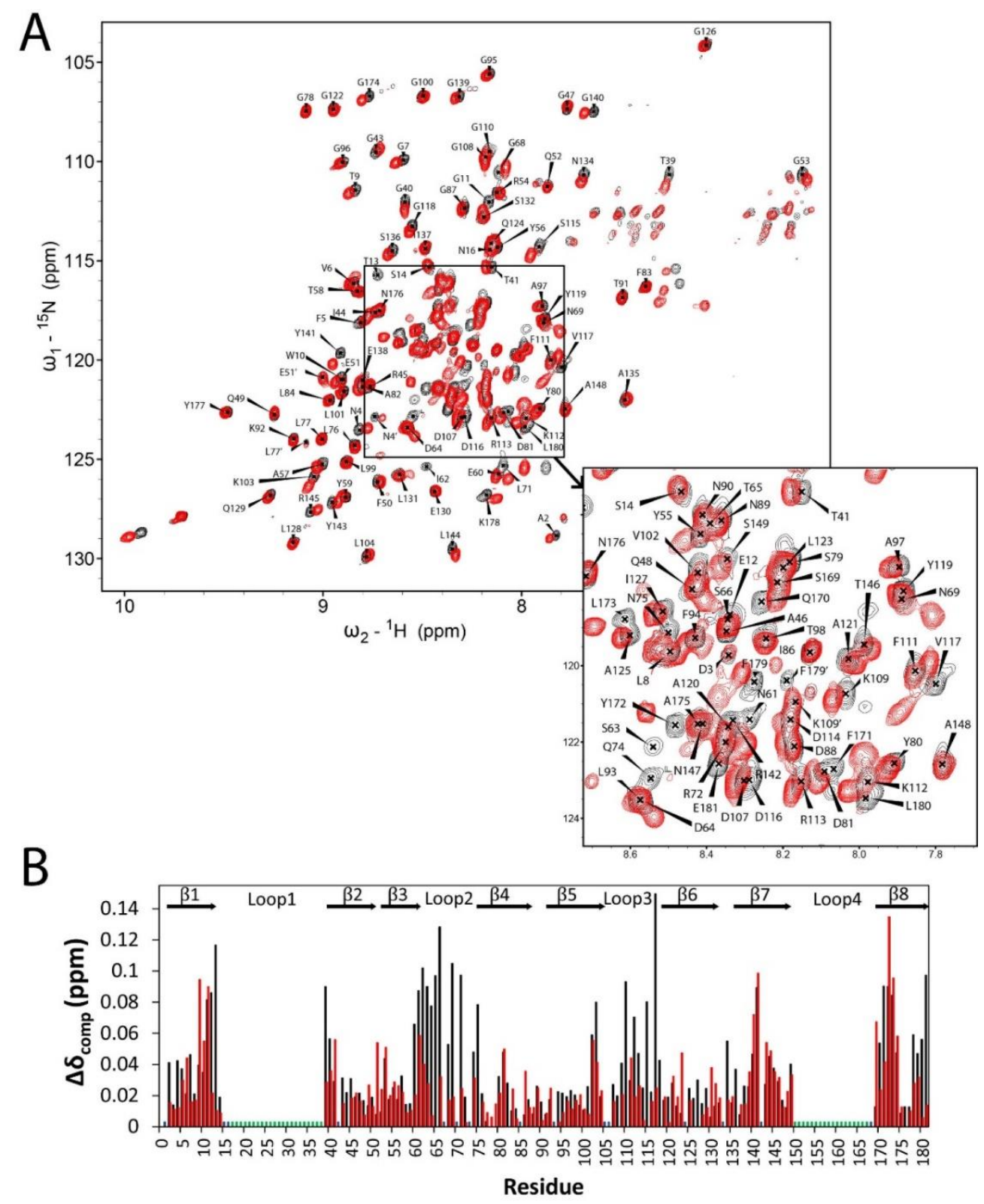

Figure 25. Chemical shift perturbations in ${ }^{15} \mathrm{~N}-{ }^{1} \mathrm{H}$ TROSY spectra upon addition of Kdo2-lipid A or LMPG to ${ }^{2} \mathrm{H}-,{ }^{13} \mathrm{C}-,{ }^{15} \mathrm{~N}$-labeled OprH $\Delta \mathrm{L} 1 \Delta \mathrm{L} 4$ in DHPC micelles. (A) ${ }^{15} \mathrm{~N}-$ ${ }^{1} \mathrm{H}$ TROSY spectrum of $0.2 \mathrm{mM}$ OprH $\Delta \mathrm{L} 1 \Delta \mathrm{L} 4$ (black) in DHPC micelles overlaid onto the spectrum of OprH $\Delta \mathrm{L} 1 \Delta \mathrm{L} 4$ in DHPC:Kdo2-lipid A mixed micelles (red, 10:1 Kdo2-lipid A:OprH molar ratio). (B) Compound chemical shift changes $\Delta \delta_{\text {comp }}=\left[\Delta \delta^{2} H N+\right.$ $\left.\left(\Delta \delta_{N} / 6.5\right)^{2}\right]^{1 / 2}$ ) resulting from the addition of $2 \mathrm{mM}$ LMPG (red) and $2 \mathrm{mM}$ Kdo2-lipid (black) relative to the chemical shifts of $0.2 \mathrm{mM} \mathrm{OprH} \Delta \mathrm{L} 1 \Delta \mathrm{L} 4$ in DHPC only. Unassigned residues are marked with blue ticks, and removed loop residues are marked with green ticks. 

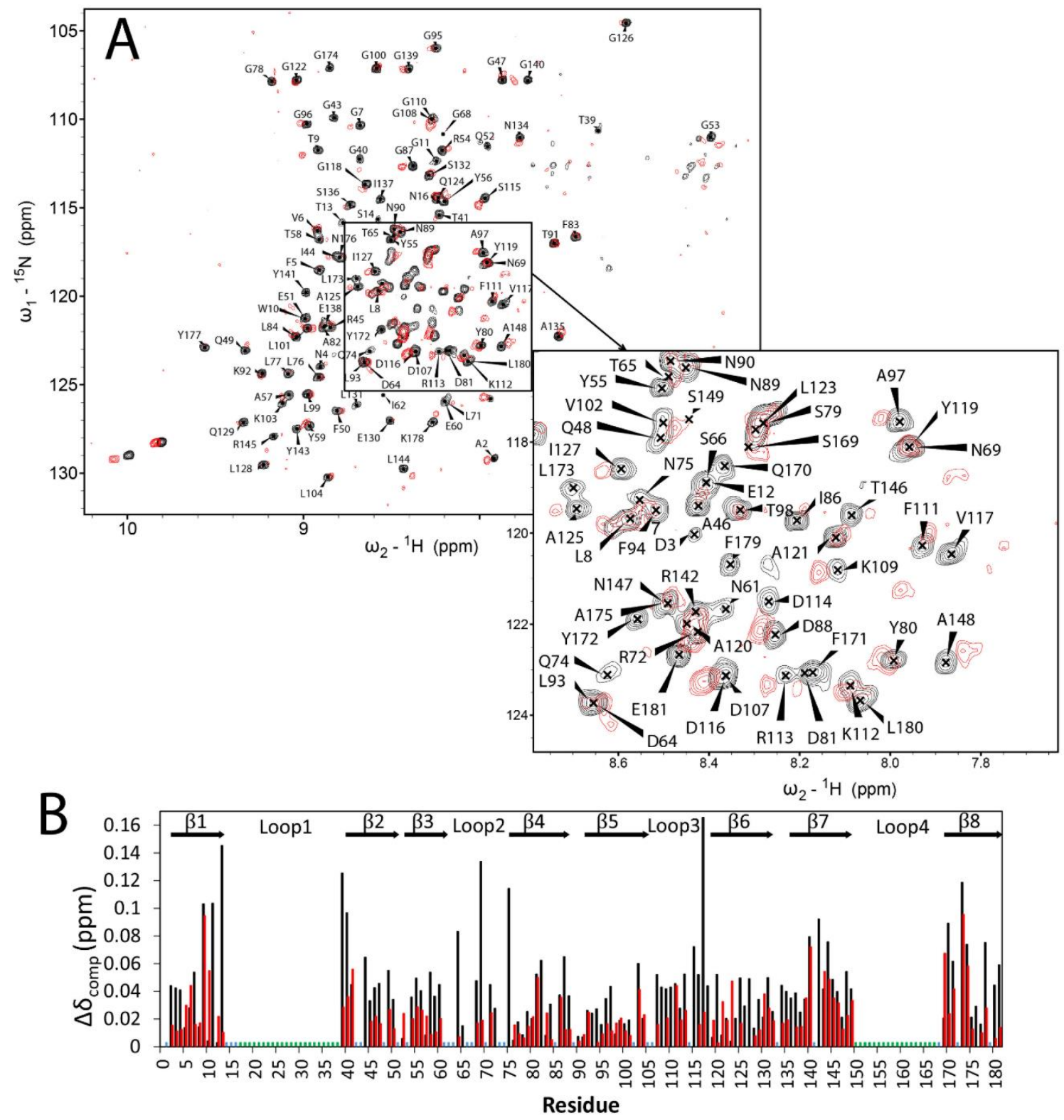

Figure 26. Chemical shift perturbations in ${ }^{15} \mathrm{~N}-{ }^{1} \mathrm{H}$ TROSY spectra upon addition of Rd2 LPS from E. coli F583 to ${ }^{2} \mathrm{H}-,{ }^{13} \mathrm{C}-,{ }^{15} \mathrm{~N}$-labeled OprH $\Delta \mathrm{L} 1 \Delta \mathrm{L} 4$ in DHPC micelles. (A) ${ }^{15} \mathrm{~N}-{ }^{1} \mathrm{H}$ TROSY spectrum of $0.2 \mathrm{mM}$ OprH $\Delta \mathrm{L} 1 \Delta \mathrm{L} 4$ (black) in DHPC micelles overlaid onto the spectrum of OprH $\Delta \mathrm{L} 1 \Delta \mathrm{L} 4$ in DHPC:Rd2 LPS mixed micelles (red, 10:1 Rd2 LPS:OprH molar ratio). (B) Compound chemical shift changes $\left.\Delta \delta_{\text {comp }}=\left[\Delta \delta^{2}{ }_{H N}+\left(\Delta \delta_{N} / 6.5\right)^{2}\right]^{1 / 2}\right)$ resulting from the addition of $2 \mathrm{mM}$ Rd2 LPS (black) relative to the chemical shifts of $0.2 \mathrm{mM}$ OprH $\Delta \mathrm{L} 1 \Delta \mathrm{L} 4$ in DHPC only. Unassigned residues are marked with blue ticks, and removed loop residues are marked with green ticks. 
micelles to a final ratio of $10: 1 \mathrm{Kdo} 2$-lipid $\mathrm{A}$ to $\mathrm{OprH} \Delta \mathrm{L} 1 \Delta \mathrm{L} 4$. The overlay of this spectrum onto the spectrum of $\mathrm{OprH} \Delta \mathrm{L} 1 \Delta \mathrm{L} 4$ in $\mathrm{DHPC}$, revealed many changes of back-bone chemical shifts (Fig. 25 A and B). Addition of Rd2 LPS from E. coli to OprH $\Delta$ L1 $\Delta$ L4 (Fig. 26 A and $\mathbf{B}$ ) resulted in similar chemical shifts as observed with Kdo2-lipid A. However, Rd LPS also caused more significant broadening of many resonance lines compared to Kdolipid A. The ${ }^{15} \mathrm{~N}-{ }^{1} \mathrm{H}$ TROSY spectra of OprH $\Delta \mathrm{L} 1 \Delta \mathrm{L} 4$ in DHPC and in DHPC:Kdo2-lipid A micelles were assigned, by recording TROSY versions of HNCA, HNCB and HNCO experiments. The resulting chemical shift perturbations are very similar to those observed with wild-type OprH (data not shown). The most significant chemical shift changes (>0.05 ppm) are found at the base of loop 1 (residues G11, E12, T13, T39, G40), in loop 2 (residues E60-L71 and N75), at the base of loop 3 (residues V102 and K103 and residues K112, R113, S115, and V117), and in $\beta$-strand 8 (residues Q170-G174 and F179E181). Addition of 2 mM 1-myristoyl-2-hydroxy-sn-glycero-3-phospho-(1'-rac-glycerol) (LMPG, Avanti), which is a negatively charged lipid with the same acyl chain length as Kdo2-lipid A, to OprH $\Delta \mathrm{L} 1 \Delta \mathrm{L} 4$ resulted in only minor chemical shift perturbations in these regions. This indicates that the perturbations resulting from the addition of Kdo2-lipid A are specific to Kdo-Lipid $\mathrm{A}$ and not the generic result of its negative charge or hydrophobic acyl chains.

To obtain more insight into interactions between the hydrophobic amino acid side chains of $\mathrm{OprH} \Delta \mathrm{L} 1 \Delta \mathrm{L} 4$ and the acyl chains of lipid A, Kdo2-lipid A was added to a sample of $0.2 \mathrm{mM}\left[\mathrm{U}-{ }^{2} \mathrm{H},{ }^{15} \mathrm{~N}\right]$; Ile $\delta 1-\left[{ }^{13} \mathrm{CH}_{3}\right]$; Leu,Val- $\left[{ }^{13} \mathrm{CH}_{3},{ }^{12} \mathrm{CD}_{3}\right]$-labeled OprH $\Delta \mathrm{L} 1 \Delta \mathrm{L} 4$ 

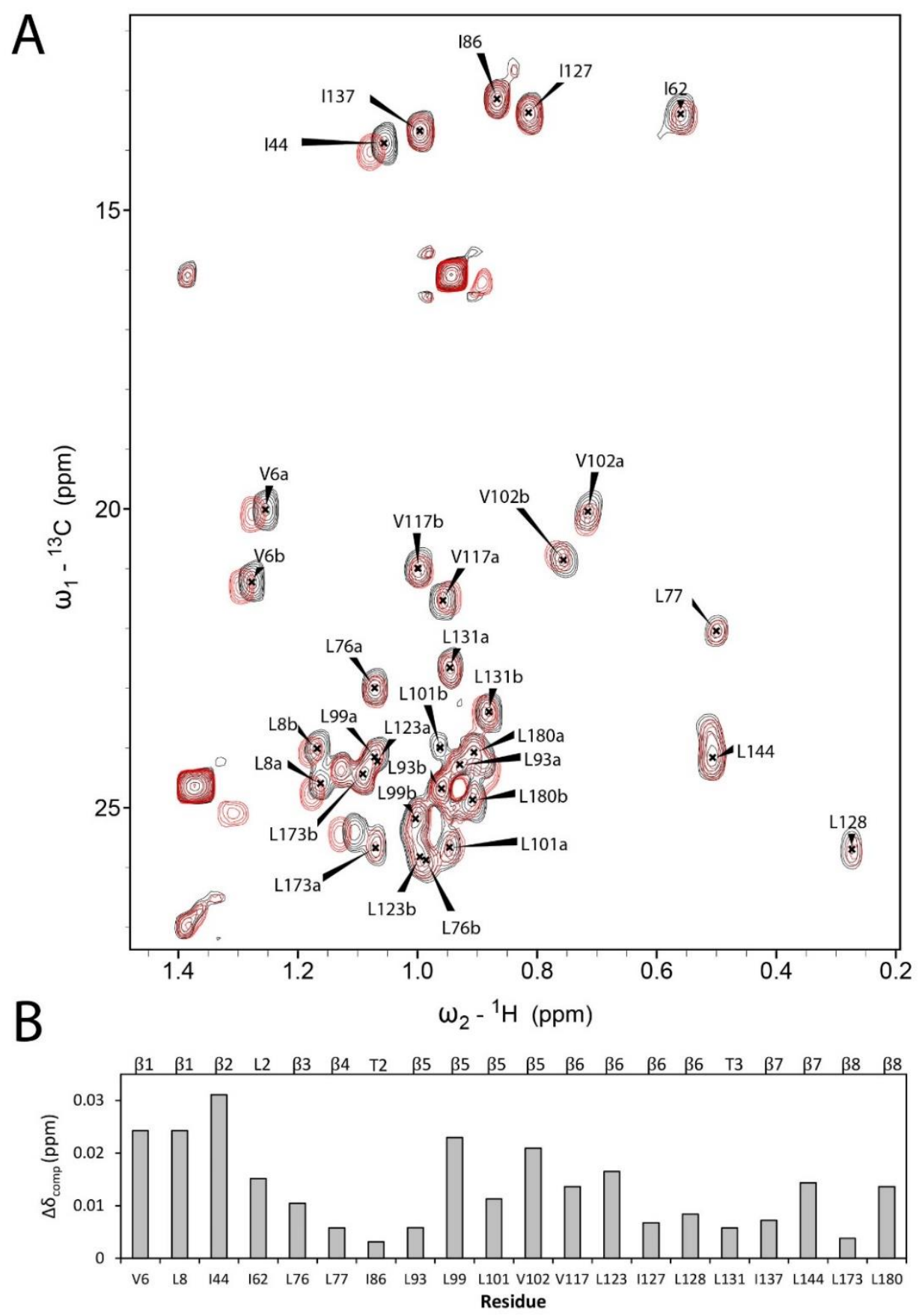

Figure 27. Chemical shift perturbations in ${ }^{1} \mathrm{H}-{ }^{13} \mathrm{C}$ HMQC spectra upon addition of Kdo2-lipid A to $\left\{\left[\mathrm{U}-{ }^{2} \mathrm{H}-,{ }^{15} \mathrm{~N}\right]\right.$; Ile $\delta 1-\left[{ }^{13} \mathrm{CH}_{3}\right]$; Leu, Val- $\left.\left[{ }^{13} \mathrm{CH}_{3},{ }^{12} \mathrm{CD}_{3}\right]\right\}$ labeled OprH $\Delta \mathrm{L} 1 \Delta \mathrm{L} 4$ in DHPC micelles. (A) ${ }^{1} \mathrm{H}-{ }^{13} \mathrm{C}$ HSQC spectrum of $0.5 \mathrm{mM}$ OprH $\Delta \mathrm{L} 1 \Delta \mathrm{L} 4$ (black) in DHPC micelles overlaid onto the spectrum of OprH $\Delta \mathrm{L} 1 \Delta \mathrm{L} 4$ in DHPC:Kdo2-lipid A mixed micelles (red, 10:1 Kdo2-lipid A:OprH molar ratio). (B) Compound chemical shift changes $\left.\Delta \delta_{\text {comp }}=\left[\Delta \delta^{2}{ }_{H}+\left(\Delta \delta_{c} / 5.4\right)^{2}\right]^{1 / 2}\right)$ resulting from the addition of $2 \mathrm{mM} \mathrm{Kdo2-lipid}$ relative to the chemical shifts of $0.2 \mathrm{mM} \mathrm{OprH} \Delta \mathrm{L} 1 \Delta \mathrm{L} 4$ in DHPC only. 
in DHPC micelles to a final ratio of $10: 1 \mathrm{Kdo}$-lipid $A$ to protein and the ${ }^{1} \mathrm{H}-{ }^{13} \mathrm{C}$ HSQC spectrum of this complex was collected (Fig. 27 A and B). The resonances of the methyl groups of these side chains can be assigned by correlation to the backbone amide resonances of the following residues using a combination of $\mathrm{H}(\mathrm{CCO}) \mathrm{NH}-\mathrm{TOCSY}$ and $(\mathrm{H}) \mathrm{C}(\mathrm{CO}) \mathrm{NH}-\mathrm{TOCSY}$ spectra. This strategy allowed us to unambiguously assign the methyl groups of all isoleucines, all valines, and 12 of the 15 leucines. The overlay of the ${ }^{1} \mathrm{H}-{ }^{13} \mathrm{C}$ HSQC spectrum recorded at a 10:1 Kdo2-lipid A:OprH ratio onto the spectrum of $\operatorname{OprH} \Delta \mathrm{L} 1 \Delta \mathrm{L} 4$ in DHPC only, revealed multiple side-chain chemical shift perturbations in $\beta$-strand 1 , the base of loop 3 and $\beta$-strand 8 . Only one loop residue, lle62 of loop 2 , was observed to show a moderate chemical shift perturbation. In general, the resulting pattern of side chain chemical shift perturbations closely follows the one obtained for the backbone resonances that were observed in OprH $\Delta \mathrm{L} 1 \Delta \mathrm{L} 4{ }^{15} \mathrm{~N}-{ }^{1} \mathrm{H}$ TROSY spectrum upon addition of Kdo2-lipid A. We also collected the ${ }^{1} \mathrm{H}-{ }^{13} \mathrm{C} H S Q C$ spectrum of $\left[\mathrm{U}-{ }^{2} \mathrm{H},{ }^{15} \mathrm{~N}\right]$;

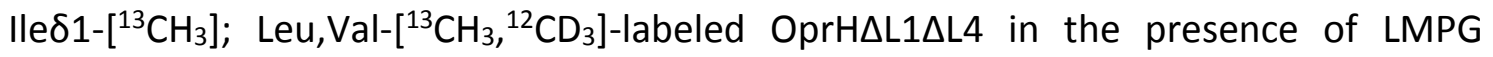
instead of Kdo2-lipid A. This spectrum appeared to be identical to the spectrum of $\operatorname{OprH} \Delta \mathrm{L} 1 \Delta \mathrm{L} 4$ in the absence of $\mathrm{LMPG}$, confirming that the observed Kdo-Lipid $A$ interactions are specific to this lipid (data not shown).

Since $\mathrm{Kdo}_{2}$-lipid A contains two phosphate groups which impart a net negative charge on the molecule, it is reasonable to assume that these lipid phosphates interact electrostatically with the side chains of basic amino acid residues. A comparison of the amino acid sequences of OprH homologs from different Pseudomonads reveals a high 


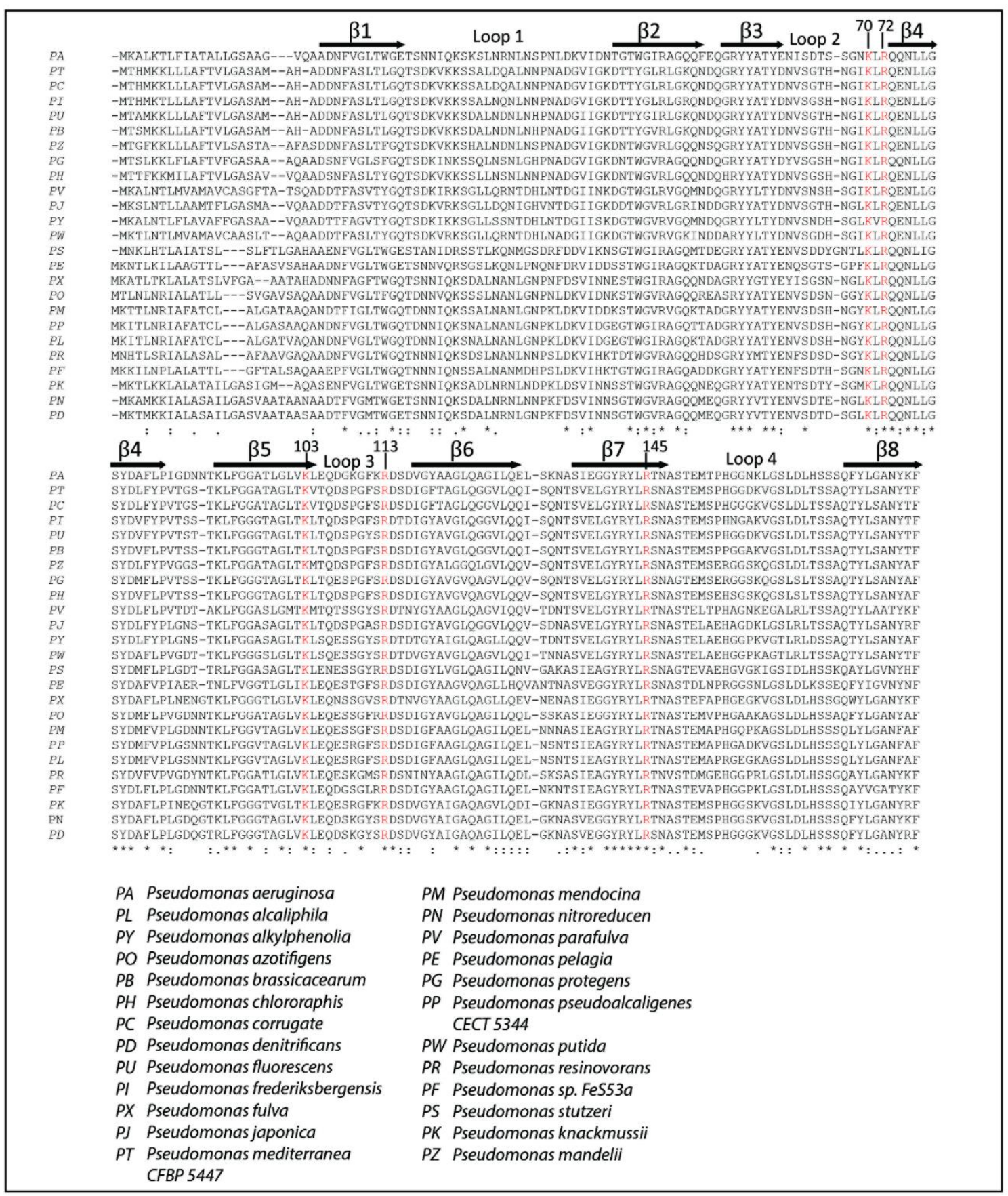

Figure 28. Clustal Omega alignment of OprH from $P$. aeruginosa and other closely related outer membrane proteins. Absolutely conserved residues are marked in the bottom line with "*", highly conserved residues with ":", and partially conserved with ".".Absolutely conserved Lys and Arg residues localized in loops 2 and 3 and in strand 7 are highlighted in red. $\beta$-strands are indicated with arrows above the sequences. 
degree of similarity, with several conserved regions, especially in the $\beta$-strands (Fig. 28). Of the seven basic residues that are localized at the extracellular upper rim of the $\beta$ barrel, five, namely K70, R72, K103, R113, and R145 are absolutely conserved. To test the hypothesis that LPS interacts with basic residues at the barrel rim or in loops 2 and 3, we collected $\mathrm{H}(\mathrm{C})(\mathrm{CC})$-TOCSY-(CO)- $\left[{ }^{15} \mathrm{~N},{ }^{1} \mathrm{H}\right]-$ TROSY spectra of a ${ }^{13} \mathrm{C}-,{ }^{15} \mathrm{~N}$-labeled $\operatorname{OprH} \Delta \mathrm{L} 1 \Delta \mathrm{L} 4$ sample with and without 10 -fold excess of Kdo2-lipid A (Fig. 29). A subset

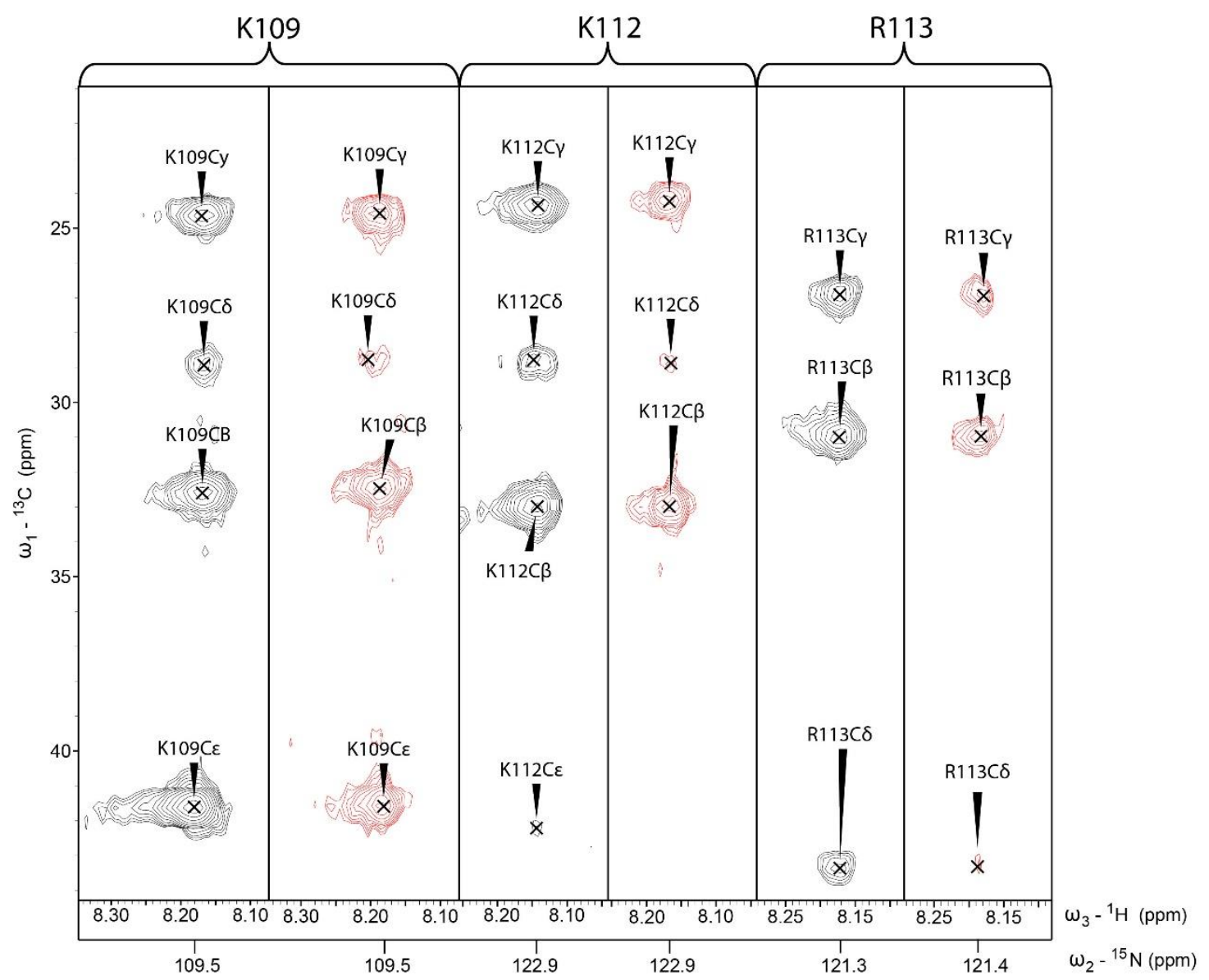

Figure 29. [ $\left[\omega_{1}\left({ }^{13} \mathrm{C}\right), \omega_{3}\left({ }^{1} \mathrm{H}\right)\right]$ strips from 3D $\mathrm{H}(\mathrm{C})(\mathrm{CC})$-TOCSY-(CO)-[ $\left.{ }^{15} \mathrm{~N},{ }^{1} \mathrm{H}\right]-T R O S Y$ spectra of ${ }^{13} \mathrm{C}-,{ }^{15} \mathrm{~N}$ labeled OprH $\Delta \mathrm{L} 1 \Delta \mathrm{L} 4$ in DHPC micelles (black) and ${ }^{13} \mathrm{C}-,{ }^{15} \mathrm{~N}$ labeled OprH $\Delta \mathrm{L} 1 \Delta \mathrm{L} 4$ in DHPC:Kdo2-lipid A mixed micelles (red, 10:1 Kdo2-lipid A:protein molar ratio). The strips were taken at the ${ }^{15} \mathrm{~N}$ chemical shifts indicated at the bottom of the strips. 
of side chains could be observed in this experiment, including the side chains of K109, $\mathrm{K} 112$ and R113, which all reside in loop 3 (Fig. 23). The side chains of the remaining basic residues could not be detected due to line-broadening. Adding Kdo2-lipid A resulted in further line-broadening and decrease in peak intensity of some, but not all residues. The side chain chemical shifts of K109 and K112 were not changed upon the addition of Kdo2-lipid A (Fig. 29). However K112 experienced a more significant decrease of the intensity of some cross-peaks, whereas those of K109 were hardly changed. The most significant changes were observed with the side chain resonances of R113. The resonances of this residue showed significant shifts in the ${ }^{15} \mathrm{~N}$ dimension and the largest decrease of the side chain resonance intensities (Fig. 29). Interestingly, the more strongly interacting rim residue R113 belongs to the absolutely conserved basic residues, whereas the more weakly interacting residues $\mathrm{K} 109$ and $\mathrm{K} 112$ are localized in loop 3 and do not appear to be conserved in OprH homologs of Pseudomonads (Fig. 28).

3.3.3 Measurement of OprH - LPS Interactions by ELISA. To measure the binding of OprH to LPS we adapted a solid-phase enzyme-linked immunosorbent assay by immobilizing Rd2 LPS on a microtiter plate and measuring the binding of refolded OprH in DHPC lipid micelles. Since we are not aware of the availability of an anti-OprH antibody, we first attempted to use an anti-PentaHis antibody (Qiagen) on His-tagged OprH. However, we observed a large amount of unspecific binding with this antibody, and therefore, decided to replace the C-terminal 6xHis-tag with a Strep-Tag II. High quality antibodies against this tag are commercially available. In Fig. 30 A we show the 
concentration-dependent binding of OprH-Strep-tag II to the LPS-coated microtiter plate, which saturates at around $10 \mu \mathrm{g} / \mathrm{ml}$ of OprH. We next used this assay to compare the influence of various conditions and mutations on the binding affinity of OprH to $\mathrm{Rd} 2$ LPS (Fig. 30 B). We used $1 \mu \mathrm{g} / \mathrm{ml}$ protein concentrations in these assays because the binding response is most sensitive in this low concentration regime.

When any of the assay components were omitted, a $450 \mathrm{~nm}$ absorbance of less than 0.06 developed in the microtiter well (Fig. 30 B, first lane) compared to more than 0.16 absorbance units for $1 \mu \mathrm{g} / \mathrm{ml}$ wild-type $\mathrm{OprH}$ in the presence of all assay components (Fig. $30 \mathrm{~B}$, second lane). Heat-denatured $\mathrm{OprH}\left(90^{\circ} \mathrm{C}, 30 \mathrm{~min}\right)$ yielded 0.07

A

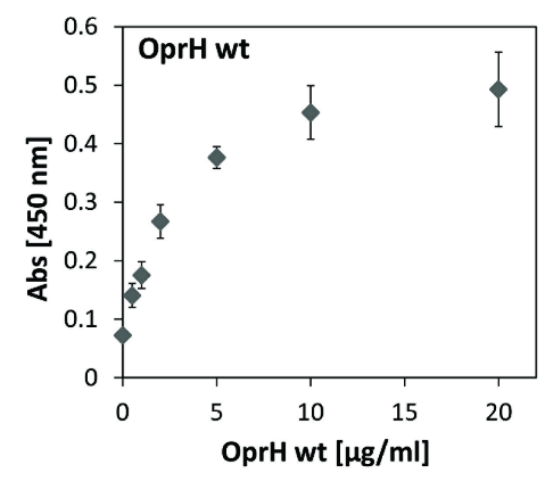

B

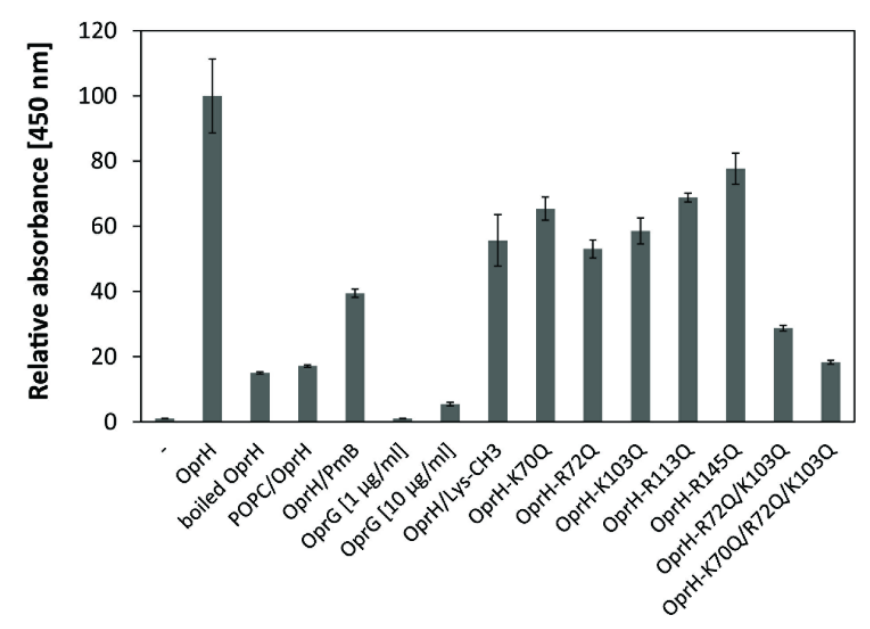

Figure 30. Binding of OprH to LPS-coated surface. (A) Concentration dependence of OprH binding to LPS-coated microtiter plate. (B) Comparison of the binding of OprH and OprH mutants to LPS-coated microtiter plates. All proteins were used at $1 \mu \mathrm{g} / \mathrm{ml}$ concentration, with exception of OprG, which was used at both 1 and $10 \mu \mathrm{g} / \mathrm{ml}$. "_. denotes no protein added, "POPC/OprH" - POPC was used to coat plates instead of LPS, "OprH/PmB" - after incubation with OprH, the plate was treated with $10 \mu \mathrm{g} / \mathrm{ml}$ polymyxin B for $30 \mathrm{~min}$, "OprH/Lys- $\mathrm{CH}_{3}$ " - OprH with methylated lysine side chains was used, OprH-K70Q, -R72Q, -K103Q, -R113Q, -R145Q, -R72Q/K103Q, -K70Q/R72Q/K103Q - OprH mutants. 
units when it was used in place of refolded OprH. Using another OMP from $P$. aeruginosa with a similar $\beta$-barrel structure, OprG, which has no known LPS binding activity, resulted in no detectable signal at both 1 and $10 \mu \mathrm{g} / \mathrm{ml}$ protein concentrations. Coating the wells with different lipids such as POPC gave little to no signal. When polymyxin B was added after OprH had bound to LPS in the well, the signal was reduced by $40 \%$, indicating that polymxyxin B is able to partially disrupt the OprH-LPS interaction or remove the OprH-LPS complex from the microtiter plate. Interestingly, the global methylation of the $\varepsilon-\mathrm{NH}_{3}$ groups in all lysines of $\mathrm{OprH}$ resulted in a $30 \%$ weaker binding compared to unmodified OprH. This suggests that not only the charge, but perhaps also the ability of lysines to form hydrogen bonds could be important for binding to LPS.

To analyze the effect of individual conserved basic residues on the ability of OprH to bind LPS, we individually mutated K70, R72, K103, R113, and R145 to glutamines. All of these single mutants showed weaker binding than wild-type (Fig. 30 B). The most significant decrease was observed with R72Q (32\% less binding) and the least significant decrease occurred with R145Q (15\% less binding). Although perhaps statistically significant only as a trend, the contributions of the individual residues to LPS binding decrease in the order $\mathrm{R} 72>K 103^{\sim} \mathrm{K} 70>\mathrm{R} 113>\mathrm{R} 145$. This result is consistent with the previous result that the removal of any single loop is not sufficient to abolish the binding of LPS to OprH [92]. However, mutation of two positive charges, namely R72Q on loop 2 and $\mathrm{K} 103 \mathrm{Q}$ on loop 3 reduced binding more than each single mutant and the triple mutant $\mathrm{K} 70 \mathrm{Q} / \mathrm{R} 72 \mathrm{Q} / \mathrm{K} 103 \mathrm{Q}$ reduced LPS binding close to the level of boiled OprH. 


\subsubsection{Mutant loop interactions and determination of dissociation constants of}

LPS - OprH binding by NMR. To determine if the previously described structural effects that LPS binding has on OprH is mitigated in these mutants with decreased binding, we expressed ${ }^{15} \mathrm{~N}$-labeled OprH-R72Q, OprH-R72Q/K103Q, and OprH-K70Q/R72Q/K103Q and collected their respective ${ }^{15} \mathrm{~N}-{ }^{1} \mathrm{H}$-TROSY spectra with and without Kdo2-lipid $\mathrm{A}$ at 10:1 lipid:protein ratios (Fig. 31). The chemical shifts of OprH-R72Q were less perturbed in the loop 2 region upon addition of Kdo2-lipid $A$ than in the wild-type protein. In case

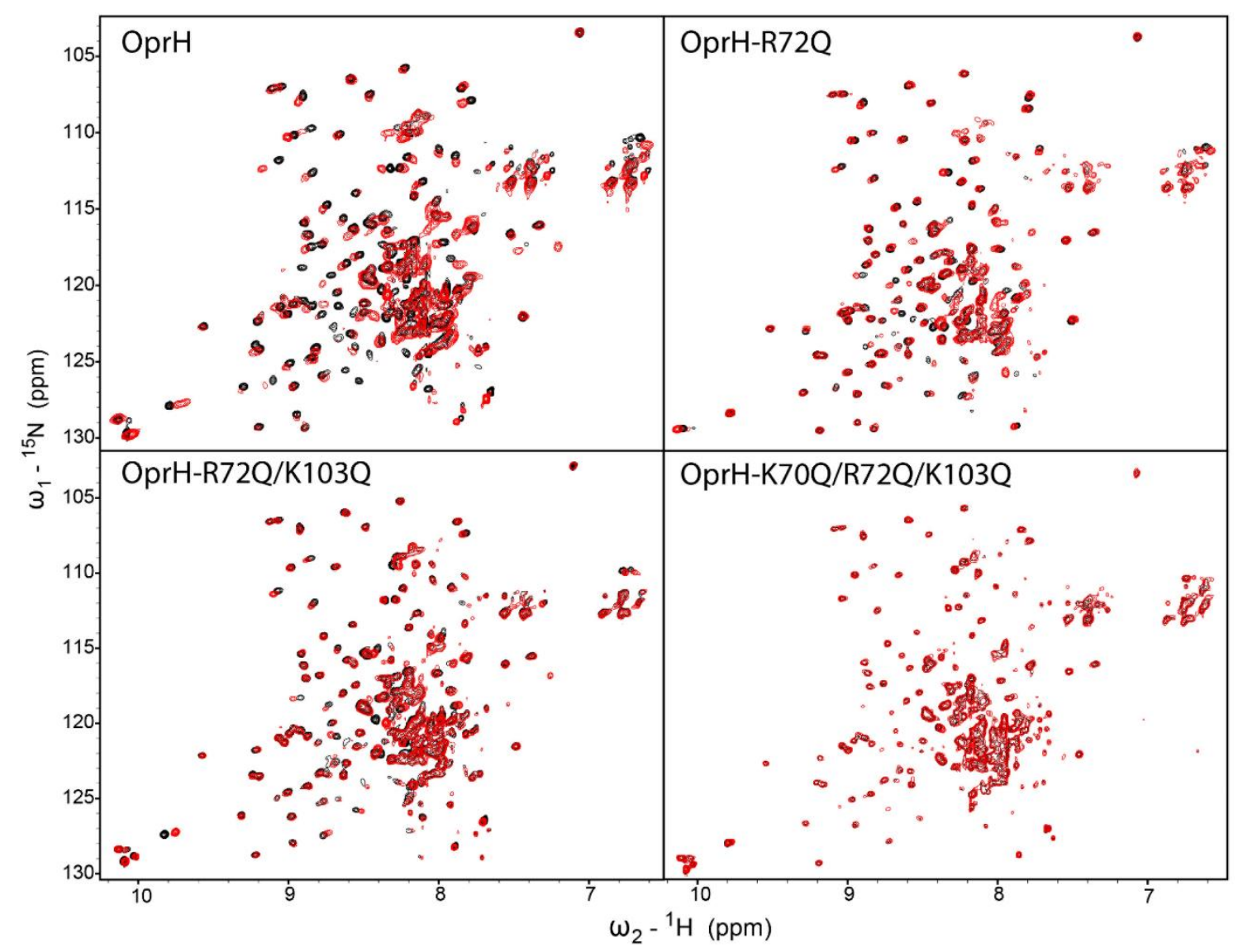

Figure 31. ${ }^{15} \mathrm{~N}-{ }^{1} \mathrm{H}$ TROSY spectra of ${ }^{15} \mathrm{~N}$-labeled OprH, OprH-R72Q, OprH-R72Q/K103Q and OprH-K70Q/R72Q/K103Q in DHPC micelles (black), overlaid onto the spectra of corresponding proteins in DHPC:Kdo2-lipid A mixed micelles at 10:1 Kdo2-lipid A:protein molar ratio (red). 


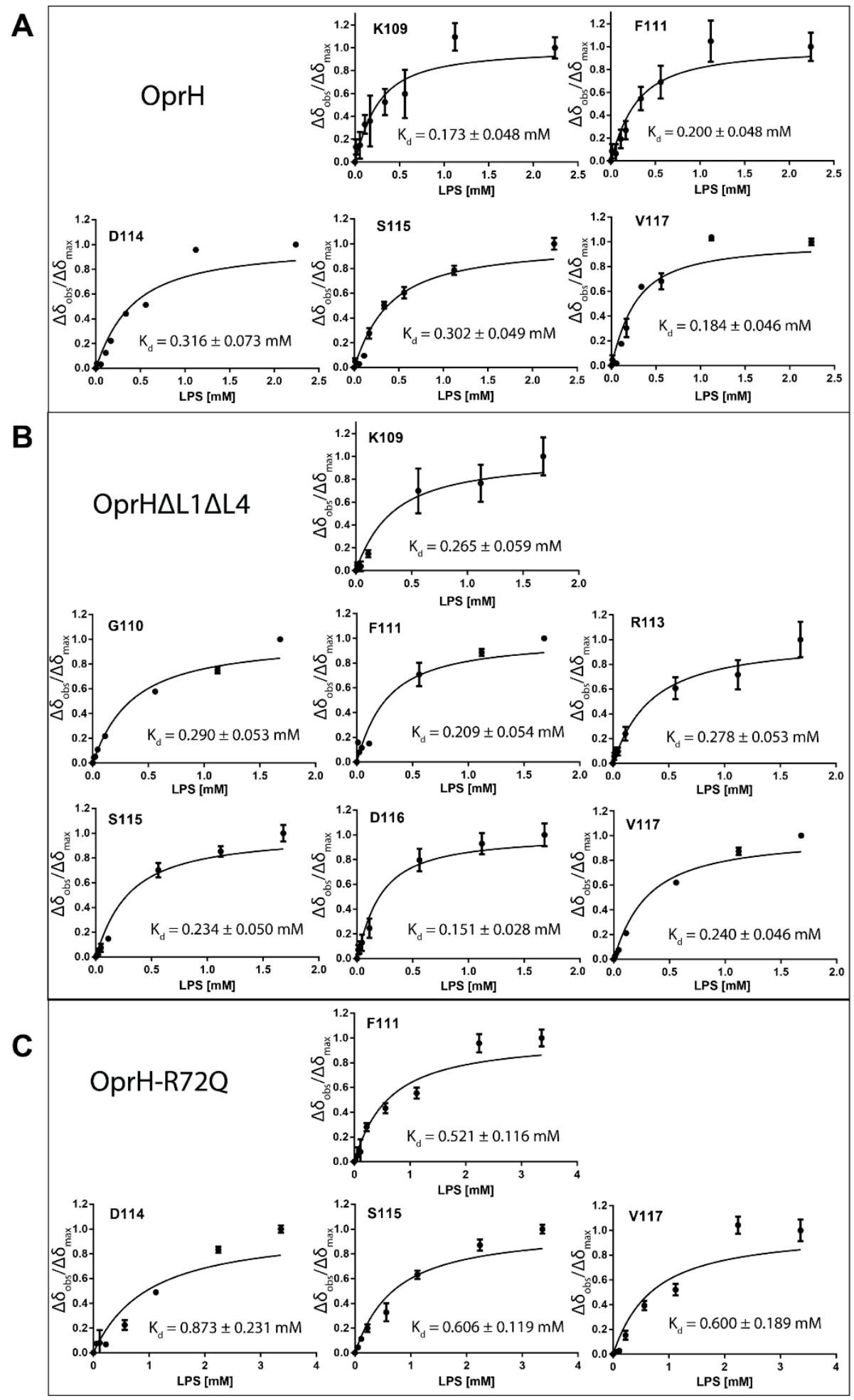

Figure 32. Binding curves for Rd2 LPS binding to different forms of OprH using the ${ }^{1} \mathrm{H}_{\mathrm{N}}$ chemical shift changes of selected loop 3 residues. (A) wt OprH, (B) OprH $\Delta \mathrm{L} 1 \Delta \mathrm{L} 4$, and (C) OprH-R72Q. 
of the double mutant OprH-R72Q/K103Q the resonances of most loop residues did not shift at all. However, OprH-K70Q/R72Q/K103Q showed a significantly different NMR spectrum with the loop resonances in the 7.6-8.4 ${ }^{1} \mathrm{H}$ ppm region significantly stronger compared to wild-type suggesting that removing all three positive charges might alter the folding and dynamics of the loops or introduces some other global changes on the protein.

To more quantitatively measure the binding of LPS to OprH, we titrated Rd2 LPS to wild-type ${ }^{2} \mathrm{H},{ }^{15} \mathrm{~N}$-labeled $\mathrm{OprH}$ and $\mathrm{OprH} \Delta \mathrm{L} 1 \Delta \mathrm{L} 4$ samples. The dissociation constant for the binding of LPS to OprH was estimated using the chemical shifts derived from the ${ }^{15} \mathrm{~N}-{ }^{1} \mathrm{H}$ TROSY cross-peaks of several loop 3 residues and equation 1 . The $\mathrm{K}_{\mathrm{d}}$ was found to be approximately $0.2 \mathrm{mM}$ for wild-type OprH (Fig. $32 \mathrm{~A}$ ) and OprH $\Delta$ L1 $\Delta$ L4 (Fig. 32 B). The R72Q mutant OprH, which showed the weakest binding of the all single mutants that were tested by ELISA, exhibited a $K_{d}$ value of approximately $0.6 \mathrm{mM}$, i.e. about a three-fold weaker binding that wild-type OprH (Fig. 32 C). These binding curves were measured using ${ }^{1} \mathrm{H}_{\mathrm{N}}$ chemical shifts of the resonances of only loop 3 cross-peaks that showed significant changes upon addition of LPS and that had no overlap with other resonances. We were unable to use resonances of loop 2 for an independent $K_{d}$ determination because the resonance lines of these cross-peaks were more overlapped and more broadened upon addition of LPS than those of loop 3. 


\section{4 Discussion}

Lipid interactions with integral membrane proteins have been studied for as long as the fluid mosaic model of biological membranes exists. While early studies debated if an annulus of distinct boundary lipids exists around integral membrane proteins [149], research has focused in more recent years on specific lipid-protein interactions. There are now over 100 crystal structures available of membrane proteins that contain density that has been interpreted as bound lipid [150]. Likewise, mass spectrometry has identified many integral membrane proteins that are ionized and desorbed from their preparation substrate with certain lipids bound [151]. But, are these lipids also specifically bound to membrane proteins in a membrane environment? Or, are they cocrystallized simply to maintain the stability of the membrane protein and are they just "hanging on" when the membrane protein "flies" through vacuum in the mass spectrometer? The answers to these questions are generally not known and it is possible, perhaps even likely, that no general answer exists. Some pairs of lipids and proteins may interact specifically when probed in membrane or micellar environments whereas others may be prompted by the special circumstances of sample handling in crystallography and mass spectrometry.

In this work, we explored the possibility to study specific lipid-protein interactions by NMR in lipid micelles in solution. The previously identified interaction between the OM lipid LPS and the Pseudomonas aeruginosa OMP OprH [92] was chosen 
for this purpose. We found that the acidic LPS binds to basic residues in loops 2 and 3 and at the extracellular rim of the $\beta$-barrel of $\mathrm{OprH}$. This electrostatic interaction is reminiscent of the interaction of the E. coli OMP FhuA with LPS [52], which to the best of our knowledge is the only other membrane protein, for which detailed molecular interactions with LPS have been reported. The structure of FhuA, which is responsible for ferric hydroxamate uptake in E. coli was solved in complex with LPS. Analysis of this structure revealed that the contacts between the protein and LPS are mainly through the phosphate residues of lipid A and Kdo I and II sugar moieties, which constitutes a region of LPS in that is highly conserved in all Gram-negative bacteria. As with OprH, strong electrostatic interactions were observed between basic, positively charged amino acids of FhuA and the negatively charged phosphates on the lipid A and inner core carbohydrate moieties of LPS. The LPS binding area covered a significant fraction of the surface of FhuA that included four strands and two extracellular loops. Our analysis of the binding interface between $\mathrm{OprH}$ and LPS reveals similar molecular interactions. The width of the OprH LPS binding site is similar to the width of the lipid A molecule (Fig. 23), and therefore it seems reasonable to assume that the LPS binding stoichiometry is $1: 1$, as is the case for LPS binding to FhuA [52] and polymyxin B [152].

By analyzing NMR chemical shift perturbations of OprH backbone and side chain resonances upon the addition of LPS, we found that the interaction is localized to the extracellular loops 2 and 3 and the base of loop 1. The resonances of numerous residues in these regions were shifted by LPS, but not by a non-LPS lipid of similar charge and 
hydrophobic acyl chain length. This suggests that the basic residues of loops 2 and 3 form hydrogen bonds with the negatively charged phosphates of the inner core region of LPS, and as a result the glucosamine backbone of lipid A is placed in close proximity to the base of extracellular loop 1. Smaller shifts in the region of $\beta$-strands 7 and 8 were not only observed with LPS, but also with LMPG. Therefore, these interactions are most likely due to unspecific hydrophobic interactions with the long acyl chains of these lipids that, when present, displace the short chain lipid DHPC.

The importance of several conserved basic residues was further supported by our ELISA binding analysis, which indicates that single mutations of arginines and lysines in loops 2 and 3 significantly decrease the binding affinity, while double and triple mutations lead to a loss of LPS binding. Interestingly, $\varepsilon-\mathrm{NH}_{3}$ methylation of the lysines of OprH also significantly decreased LPS binding, which suggests that the capability of the primary amine of lysine to form hydrogen bonds is also important and not just its charge, which is preserved in the quaternary amine of the methylated species. Alternatively, the bulkiness of the methylated side chain may be detrimental to LPS binding.

The binding of LPS to OprH with $\mathrm{K}_{d} \sim 200 \mu \mathrm{M}$ appears to be relatively weak when simply considered as a binary interaction in solution. However, the binding of LPS to its binding site on OprH is actually highly efficient if one considers the very high density of LPS in the bacterial OM. Given a local "concentration" of LPS close to $1 M$, a $K_{d}$ of a few hundred $\mu \mathrm{M}$ is by far sufficient to occupy each binding site with a LPS molecule. 
Although $\mathrm{Mg}^{2+}$ and $\mathrm{Ca}^{2+}$ bind to LPS with a $\mathrm{K}_{\mathrm{d}}$ of approximately $10 \mu \mathrm{M}$ [153], OprH takes the place of divalent cations in binding LPS under low divalent cation conditions, as has been documented physiologically [92]. This effect should be enhanced in the membrane compared to micellar solutions because the local concentrations of lipids and proteins in the membrane are orders of magnitude higher than even high concentrations of divalent cations in solution.

In this work, we have mainly used Kdo2-lipid A and Rd2 LPS from E. coli, as they have core regions that are similar to the core regions of $P$. aeruginosa smooth LPS. However, the smaller size of the $E$. coli species used in this study simplifies their use in solution NMR experiments. The most significant difference between $P$. aeruginosa LPS and $E$. coli LPS lies in their acyl chains. The most common acyl chain length is 14 carbons in case of $E$. coli and 10-12 carbons in the case of $P$. aeruginosa. In addition, $E$. coli synthetizes mainly hexa-acetylated LPS, while laboratory adapted strains of $P$. aeruginosa produce $~ 75 \%$ penta-acetylated LPS and $25 \%$ hexa-acetylated LPS $[17,18]$. P. aeruginosa strains synthetizing hepta-acetylated lipid $A$, which contain an additional palmitoyl (16:0) chain linked to the primary 3-hydroxy-decanoic acid group, have also been isolated $[18,19]$. However, because of the heterogeneity in acyl chain composition of LPS that is produced by $P$. aeruginosa, it is unlikely that the acyl chains are a key part of the LPS-OprH interaction. 
In conclusion, we have identified several key residues that are responsible for the binding of LPS to OprH. We have also established the dissociation constant for this interaction that likely contributes to antibiotic resistance during $P$. aeruginosa infections. Beyond the specific LPS-OprH interaction, this work also demonstrates the versatility of modern solution NMR, from establishing protein structure and investigating protein backbone and side chain dynamics to the identification of binding sites and dissociation constants of selectively binding membrane lipids that cannot be easily achieved by other analytical methods. 


\title{
Chapter 4. Optimizing nanodiscs and bicelles
}

\author{
for solution NMR studies of two $\beta$-barrel
}

\section{membrane proteins}

Iga Kucharska, Thomas Edrington, Binyong Liang, and Lukas K. Tamm

Journal of Biomolecular NMR, April 2015, Volume 61, Issue 3, pp 261-274

\subsection{Introduction}

Membrane proteins constitute around $30 \%$ of the human genome and are the targets of many therapeutic agents [154]. Despite their importance there is substantially less structural and functional data available about them compared to soluble proteins, mainly due to difficulties in the expression and purification of sufficient yields and their preparation in suitable membrane-mimicking environments. Membrane proteins are most frequently prepared in detergent micelles for structural analysis by $\mathrm{x}$-ray crystallography or solution NMR. However, in some detergents membrane proteins may partially or fully denature resulting in loss of activity $[155,156]$. Therefore, there is a need to find better mimics of the natural lipid bilayer membrane environment when structurally characterizing membrane proteins. 
Certain mixtures of long- and short-chain phospholipids form dynamic discshaped assemblies called lipid bicelles [157-159]. For studying membrane proteins by solution NMR spectroscopy they are frequently composed of one part of 1,2dimyristoyl-sn-glycero-3-phosphocholine (DMPC) and two to four parts of 1,2dihexanoyl-sn-glycero-3-phosphocholine (DHPC). DMPC is thought to form a central bilayered core surrounding the protein of interest while DHPC forms the edge of a discshaped assembly. However, this segregation is probably not very strict and both types of lipids are likely in fairly rapid dynamic exchange [158]. The size of lipid bicelles can be manipulated by changing the ratio of the long- to short-chain lipids, or q value, but only a relatively limited range of $q$ values from $\sim 0.25$ to 0.5 is useful for solution NMR. Successful solution NMR studies in bicelles include among others the structure determinations of the transmembrane segments of integrin $\beta 3$ [160], the transporter Smr [161], the receptor tyrosine kinase EphA1 [162], synaptobrevin-2 [163] and the amyloid precursor protein C99 [164].

A recent and very promising advance as a small-scale model of a lipid bilayer that is suitable for solution NMR studies is the development of lipid nanodiscs [165-167]. Nanodiscs are fragments of lipid bilayers surrounded by two copies of the $\alpha$-helical amphipathic apolipoprotein A-I. The presence of a defined protein belt around a small patch of lipid bilayer ensures the controlled size and monodispersity of nanodisc, which is thought to be an important advantage of this system compared to the more 
heterogeneous micelle and bicelle preparations. Importantly, nanodiscs are detergentfree, and therefore, embedded membrane proteins should not be influenced by adverse detergent effects. Several membrane proteins have been successfully reconstituted into nanodiscs and studied by solution NMR, including the helical transmembrane domains of CD4 [167], the viral proteins pf1, p7, and the G-protein-coupled receptor CXCR1 [168], bacteriorhodopsin $[129,169]$, YgaP [170], and the $\beta$-barrel membrane proteins OmpX [129], OmpA [171], VDAC1 [172] and VDAC2 [173]. However, the size of conventional nanodiscs is estimated to be $\sim 120 \mathrm{kDa}$ before the membrane protein of interest is added, which makes 3D NMR experiments very challenging, often requiring lipid deuteration and very high-field instruments to achieve the best resolution. To partially ameliorate this problem Hagn et al [129] engineered shorter versions of the standard MSP1 apolipoprotein that yielded substantially smaller nanodiscs with diameters ranging from around $6 \mathrm{~nm}$ to $10 \mathrm{~nm}$. The generation and use of these smaller nanodiscs was essential for obtaining very high-quality NMR spectra of OmpX, which led to solving the first structure of a membrane protein in nanodiscs.

Motivated by this work, we present a detailed comparison of membranemimetic conditions - using lipid micelles, bicelles and nanodiscs - to obtain spectra of good and lesser quality of two $\beta$-barrel OM proteins from Pseudomonas aeruginosa, namely OprG and OprH. Although unrelated in sequence and function, both proteins share similar topologies: they are 8 -stranded $\beta$-barrels with 4 relatively long 
extracellular loops and 3 short periplasmic turns. OprG is speculated to function as a transporter of hydrophobic substrates through the OM [74] and OprH functions to provide stability to the $\mathrm{OM}$ of $P$. aeruginosa by directly interacting with lipopolysaccharides (LPS) in the membrane [92].

\subsection{Methods}

4.2.1 Expression and purification of OprG and OprH proteins. OprG and OprH sequences were cloned from $P$. aeruginosa PAO1 DNA into a pET30a+ vector containing the T7 promoter (EMD Biosciences, Billerica, MA). Both constructs were cloned without the $\mathrm{N}$-terminal signal sequence (in OprG residues 1 to 26 were replaced with Met1 so that His27 becomes His2 in our numbering system; in OprH residues 1-22 were replaced with Met-1 so that Ala-23 becomes Ala-2 in our numbering system) and contained a Cterminal 6xHis-tag (Fig. 33). ${ }^{2} \mathrm{H}-,{ }^{15} \mathrm{~N}$-labeled P. aeruginosa OprG and OprH were expressed in E. coli strain BL21(DE3) (Agilent Technologies, Santa Clara, CA), purified and refolded as described previously for OprH [92].

4.2.2 Site-directed mutagenesis of OprH. Primers were designed to remove the loop 1 residues Ile17-Asn38 and the loop 4 residues Thr150-Ser162 from the OprH sequence (Fig. 33) using the Stratagene (Santa Clara, CA) QuikChange site-directed mutagenesis kit. Parent DNA coding for wild-type (wt) OprH and the forward and reverse primers of each desired mutation were cycled 20 times in a PCR reaction where the 
annealing step was set to $68^{\circ} \mathrm{C}$. Amplification products were digested for 1 hour with the restriction enzyme Dpnl. The DNA was transformed using XL1-Blue supercompetent cells from Agilent Technologies according to manufacturer's recommended protocol. The resulting loop deletion construct $(\mathrm{OprH} \Delta \mathrm{L} 1 \Delta \mathrm{L} 4)$ was expressed and refolded using the same methods as wt OprH.

\section{OprG}

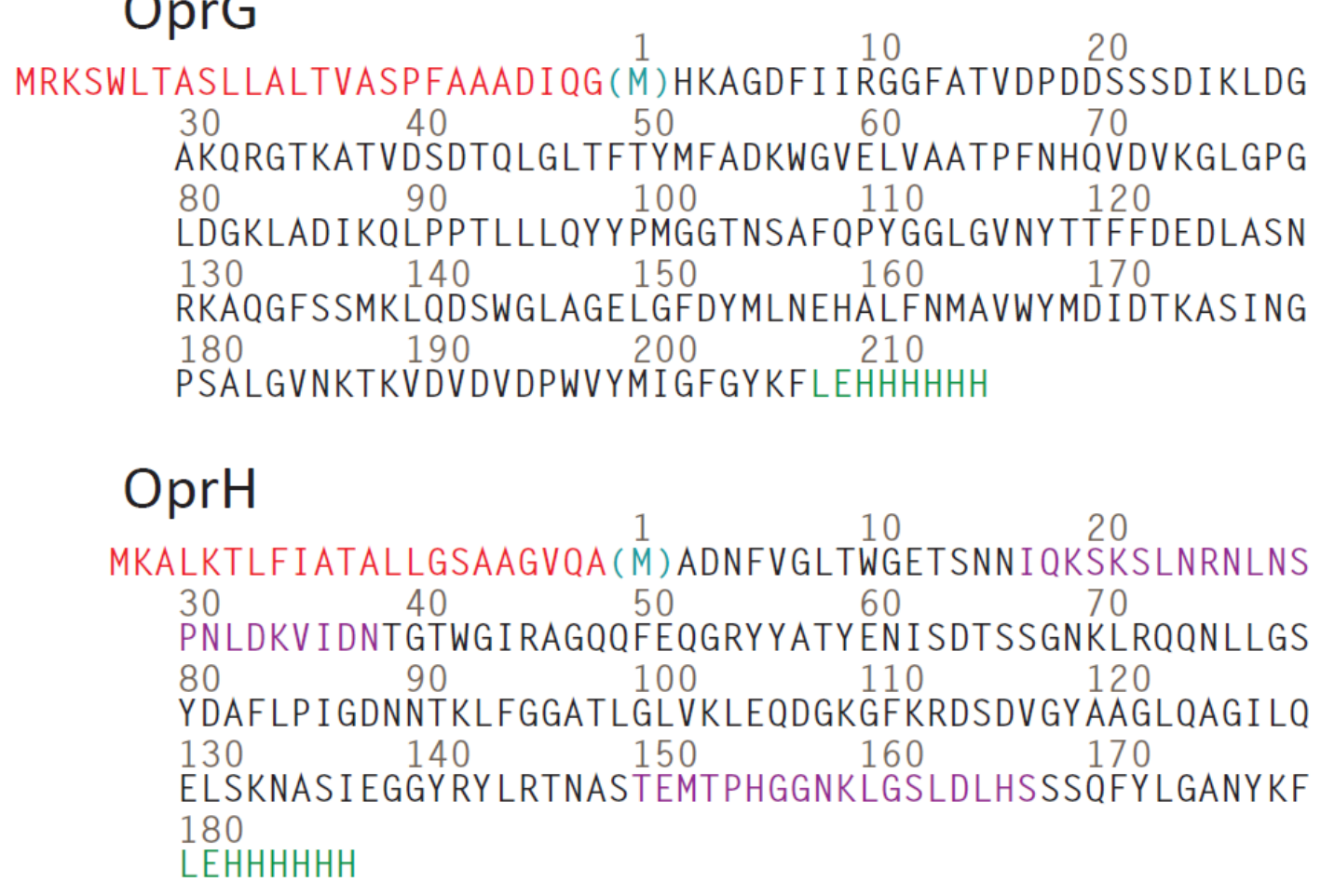

Figure 33. Amino-acid sequences of Pseudomonas aeruginosa outer membrane proteins used in this study. Removed signal sequences are depicted in red, introduced methionines are highlighted in blue, 6xHis-tag and additional amino-acid residues introduced from Pet30a+ plasmid are depicted in green. To create OprH $\Delta \mathrm{L} 1 \Delta \mathrm{L} 4$ construct we removed residues from loops 1 and 4 that are highlighted in purple.

4.2.3 Expression and purification of MSP proteins. The plasmids for all membrane scaffold protein (MSP) constructs were obtained from Dr. Gerhard Wagner (Harvard Medical School). E. coli strain BL21(DE3) competent cells were transformed 
with the desired MSP plasmid. One colony from the transformation was used to inoculate $20 \mathrm{ml}$ of LB media. This preculture was grown overnight at $37^{\circ} \mathrm{C}(225 \mathrm{rpm}$ shaker speed). The $20 \mathrm{ml}$ preculture was pelleted at $25^{\circ} \mathrm{C}$ for $10 \mathrm{~min}$ at $4000 \mathrm{rpm}$ and resuspended in $1 \mathrm{~L}$ of $\mathrm{LB}$ media. $1 \mathrm{~L}$ cultures were grown at $37^{\circ} \mathrm{C}$ to an $\mathrm{OD}$ of 0.5 . After the addition of $1 \mathrm{mM}$ isopropyl $\beta$-D-1 thiogalactopyranoside (IPTG) the culture was incubated at $37^{\circ} \mathrm{C}$ for $3-4 \mathrm{hrs}$. Cells were pelleted at $6000 \mathrm{rpm}$ and $4^{\circ} \mathrm{C}$ for $15 \mathrm{~min}$ and stored at $-80^{\circ} \mathrm{C}$ or immediately purified. The cell pellets were resuspended on ice in 10 $\mathrm{ml}$ of $50 \mathrm{mM}$ Tris/ $\mathrm{HCl}$ pH 8.0, $500 \mathrm{mM} \mathrm{NaCl}, 1 \mathrm{mM}$ EDTA (Buffer A) supplemented with 1\% TritonX-100 and $100 \mu \mathrm{L}$ of Pierce (Waltham, MA) protein inhibitor cocktail. The resuspended pellets were lysed with three passes through a MP-110P microfluidizer (Microfluidics, Newton, MA) at 20,000 psi. Much of the MSP proteins were found in the insoluble fractions, depending on the expression level or the construct purified. The soluble cell fractions were separated from the insoluble inclusion bodies by centrifugation at $11,000 \times \mathrm{g}$ for $15 \mathrm{~min}$ at $4^{\circ} \mathrm{C}$. The supernatants were stored separately and inclusion bodies were resuspended in $10 \mathrm{ml}$ of Buffer A. After centrifugation at $11,000 \times \mathrm{g}$ for $15 \mathrm{~min}$ at $4^{\circ} \mathrm{C}$ the inclusion bodies were again resuspended in $10 \mathrm{ml}$ of Buffer A. This washing of the inclusion bodies was repeated three times. The washed inclusion bodies were resuspended in $10 \mathrm{ml}$ of $50 \mathrm{mM} \mathrm{NaPO}_{4} \mathrm{pH} 8.0,300 \mathrm{mM} \mathrm{NaCl}, 8$ M urea (Buffer $\mathrm{B}$ ) and rotated at $4^{\circ} \mathrm{C}$ for $2 \mathrm{hrs}$. The supernatant and solubilized inclusion bodies were combined with prewashed Ni-NTA agarose beads (Qiagen) and rotated separately at $4^{\circ} \mathrm{C}$ for $1 \mathrm{~h}$. The Ni-NTA columns were washed with $5 \mathrm{ml}$ of Buffer A 
supplemented with $1 \%$ TritonX-100 and combined. Beads were washed with 5 column volumes of Buffer A plus $50 \mathrm{mM}$ sodium cholate, 5 column volumes of Buffer $A$ and 5 column volumes of Buffer A plus 20 mM imidazole. MSPs were eluted with Buffer A plus $500 \mathrm{mM}$ imidazole and $10 \mathrm{mM}$ sodium cholate (addition of sodium cholate was optional, but we noticed it prevented protein precipitation during concentration). The eluted volume was concentrated to 10 ml using Ultra15 3,000 MWCO membranes (Millipore, Billerica, MA) and loaded on a Superdex $26 / 60$ column pre-equilibrated with $50 \mathrm{mM}$ Tris $\mathrm{pH} 8,100 \mathrm{mM} \mathrm{NaCl}, 4 \mathrm{mM} \beta$-mercaptoethanol (Buffer C). Fractions containing the proteins were pooled and concentrated to $\sim 10 \mathrm{ml}$.

4.2.4 TEV cleavage. Tobacco etch virus protease (purified following the protocol of [174]) was added to the purified MSP protein solutions ( $1 A_{280}$ unit of TEV for $100 A_{280}$ units of MSP protein) and incubated overnight at $4^{\circ} \mathrm{C}$. The supernatants were rotated at $4^{\circ} \mathrm{C}$ for $1 \mathrm{~h}$ with prewashed (Buffer C) Ni-NTA agarose beads. The beads were washed with Buffer C until MSPs were no longer detectable in the wash. The flow-through and wash fractions containing proteins were pooled and $10 \mathrm{mM}$ sodium cholate and $2 \mathrm{mM}$ EDTA were added to the protein solutions. The proteins were concentrated to $5-10 \mathrm{mg} / \mathrm{ml}$, flash frozen in liquid $\mathrm{N}_{2}$ and stored at $-80^{\circ} \mathrm{C}$. The nomenclature adopted here and similarly elsewhere (Hagn et al 2013; Schuler et al. 2013) designates MSPs with cleaved N-terminal His-tags MSP1D1 $(\Delta x)$ and those with uncleaved Ntermini $\operatorname{MSP} 1(\Delta \mathrm{x})$ 
4.2.5 Nanodisc assembly. Nanodiscs were assembled following established protocols $[129,166,175]$. DMPC solubilized in sodium cholate (at 2:1 cholate/DMPC ratio), MSP and OprG or OprH were mixed in MSP buffer (20 mM Tris/HCl pH 7.5, 100 $\mathrm{mM} \mathrm{NaCl}, 5 \mathrm{mM}$ EDTA, $0.01 \%$ sodium azide) at a ratio of $60 \mu \mathrm{M}$ OprG or OprH to 240 $\mu \mathrm{M}$ MSP. The MSP-to-lipid ratios were 1:65, 1:40, and 1:20 for MSP1D1, MSP1D1 $\Delta$ H4 or $\Delta \mathrm{H} 5$, and MSP1D1 $\mathrm{H} 4 \Delta \mathrm{H} 5$ or MSP1D1 $\Delta \mathrm{H} 4-\Delta \mathrm{H} 6$, respectively, as proposed for the similarly-sized protein OmpX (Hagn et al 2013). After incubation at $4^{\circ} \mathrm{C}$ for two hours, 1 to $1.5 \mathrm{~g}$ of wetted Biobeads SM-2 (Biorad, Hercules, CA) were added per $1 \mathrm{ml}$ of the assembly mixture and the suspension was rotated overnight at room temperature. The assembly mixture with Bio-Beads was applied on a gravity column and washed with $1 x$ volume MSP buffer. Since OprH and OprG contain C-terminal 6xHis-tags, it is possible to separate empty from OprH- or OprG-containing nanodiscs by binding the particles to $\mathrm{Ni}$ NTA beads. The flow through and washes from the Bio-Beads were combined and mixed with prewashed Ni-NTA beads and incubated with mixing for $1 \mathrm{hr}$ at $4^{\circ} \mathrm{C}$ and applied to a gravity column. The flow throughs were discarded and the OprH- or OprG-containing nanodiscs were eluted with MSP buffer plus $300 \mathrm{mM}$ imidazole. The eluate was concentrated to $0.5 \mathrm{ml}$ using an Amicon centrifugal unit of $10 \mathrm{kDa}$ MWCO (Millipore), filtered and loaded on a Superdex 200 10/30 size exclusion column that was equilibrated with $20 \mathrm{mM}$ sodium phosphate $\mathrm{pH} 6.5,50 \mathrm{mM} \mathrm{KCl}, 1 \mathrm{mM}$ EDTA, $0.01 \%$ sodium azide. Fractions containing OprH or OprG nanodiscs were combined and concentrated using 
an Amicon centrifugal unit of $10 \mathrm{kDa}$ MWCO. The final NMR samples contained $\sim 0.5 \mathrm{mM}$ of ${ }^{2} \mathrm{H},{ }^{15} \mathrm{~N}$-labeled OprG or OprH.

\subsubsection{Preparation of $q=0.3$ bicelles composed of DHPC:DMPC:DHPS:DMPS}

27:9:3:1. All lipids were purchased from Avanti Polar Lipids (Alabaster, $A L$ ) or Anatrace (Maumee, $\mathrm{OH})$. Bicelles were prepared according to the established protocols $[159,176-$ 178]. DHPC concentrations in refolded ${ }^{2} \mathrm{H}-,{ }^{13} \mathrm{C}-,{ }^{15} \mathrm{~N}$-labeled $\mathrm{OprG}$ and OprH samples were measured by ${ }^{1} \mathrm{H}-\mathrm{NMR}$ with sodium 2,2-dimethyl-2-silapentane-5-sulfonate (DSS) as a standard. Appropriate amounts of solid 1,2-dihexanoyl-sn-glycero-3-phospho-Lserine (DHPS) were added to the protein samples and left for an hour at room temperature. The protein sample in DHPC/DHPS was added to a glass vial containing appropriate amounts of a nitrogen-dried DMPC and 1,2-dimyristoyl-sn-glycero-3phospho-L-serine (DMPS) mixture. To solubilize and equilibrate the lipids, the whole protein/detergent/lipid mixture was frozen and thawed 3 times (cycles of 40 min on ice and $40 \mathrm{~min}$ at $\left.40^{\circ} \mathrm{C}\right)$.

4.2.7 NMR Spectroscopy. All NMR experiments were recorded at $45^{\circ} \mathrm{C}$ on a Bruker Avance III 800 spectrometer equipped with a triple-resonance cryoprobe. The 1D TRACT pulse scheme was used to determine rotational correlation times [119]. Experiments were performed using the Bruker Topspin version 2.1.6 software suite.

Sequential backbone assignments for ${ }^{2} \mathrm{H}-,{ }^{13} \mathrm{C}-,{ }^{15} \mathrm{~N}$-labeled $\mathrm{OprH}$ in bicelles were 
obtained by recording TROSY versions of HNCA, HNCB, HNCO and HNCACO experiments. The NMR data were processed with NMRPipe [179] and analyzed using Sparky [111].

\subsection{Results}

4.3.1 Protein expression and purification. To examine the effect of decreasing the size of nanodiscs on the quality of the NMR spectra of incorporated membrane proteins, deletion mutants of the standard membrane scaffold protein MSP1D1 [166] were overexpressed in E. coli following the protocol of Hagn et al [129]. All proteins showed good levels of expression (Fig. 34). However, we encountered some problems during their purification, which included occasional precipitation and degradation of some of the proteins. Therefore, we modified the original protocol and used gel

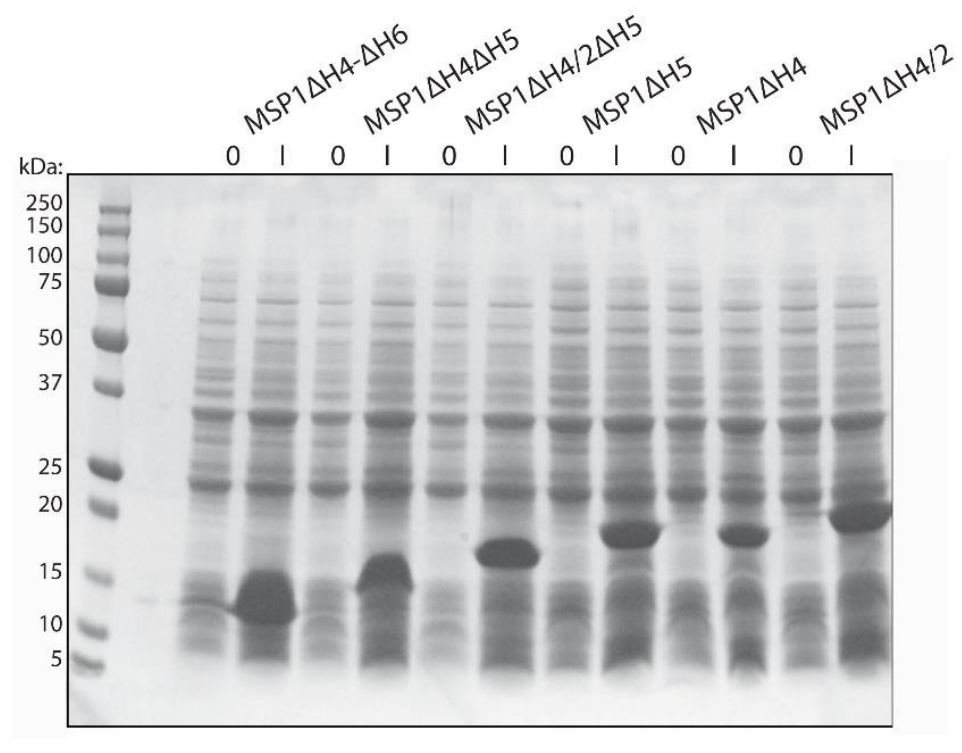

Figure 34. Expression of different MSP constructs. SDS-PAGE of samples of BL21 cells transformed with MSP constructs before (0) and $3 \mathrm{hrs}$ after induction with IPTG (I). 
filtration rather than dialysis after affinity chromatography, which in our hands resulted in shorter times for purification, higher purities, and better protein stability (Fig. 35). We also found that the shortened mutants of MSP1D1 tended to precipitate during concentration, but the addition of cholate remedied this problem and stabilized the proteins. The OprG and OprH OM proteins were overexpressed in E. coli with C-terminal His-tags and with their signal sequences deleted (Fig. 34). Both proteins were expressed

A

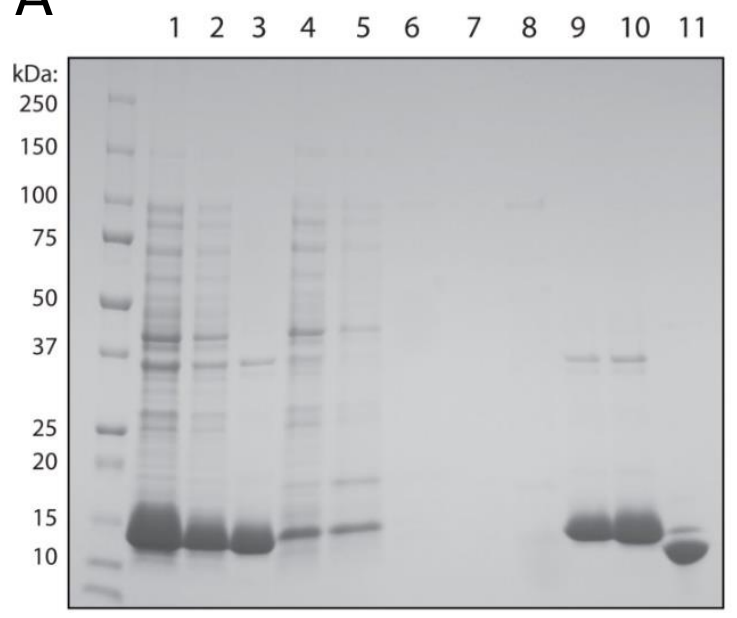

B

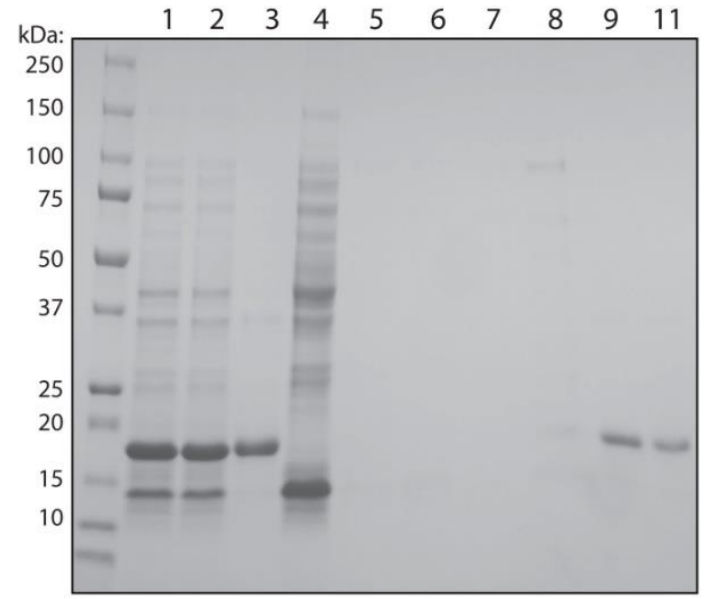

Figure 35. SDS-PAGE analysis of MSP1D1 $\Delta \mathrm{H} 4-\Delta \mathrm{H} 6(\mathrm{~A})$ and MSP1D1 $\Delta \mathrm{H} 5$ (B) purification. 1 - lysed cells, 2 - soluble fraction, 3 - inclusion bodies, 4 - flow from Ni-NTA column, 5 $1^{\text {st }}$ wash of Ni-NTA column, $6-2^{\text {nd }}$ wash of Ni-NTA column, $7-3^{\text {rd }}$ wash of Ni-NTA column, $8-4^{\text {th }}$ wash of Ni-NTA column, 9 - elution from Ni-NTA column , 10 - sample after gel filtration purification , 11 - TEV-cleaved sample.

into inclusion bodies in high yields and solubilized in urea before being purified by $\mathrm{Ni}^{2+}$ affinity chromatography. Purified proteins were then refolded into DHPC micelles for further use. 


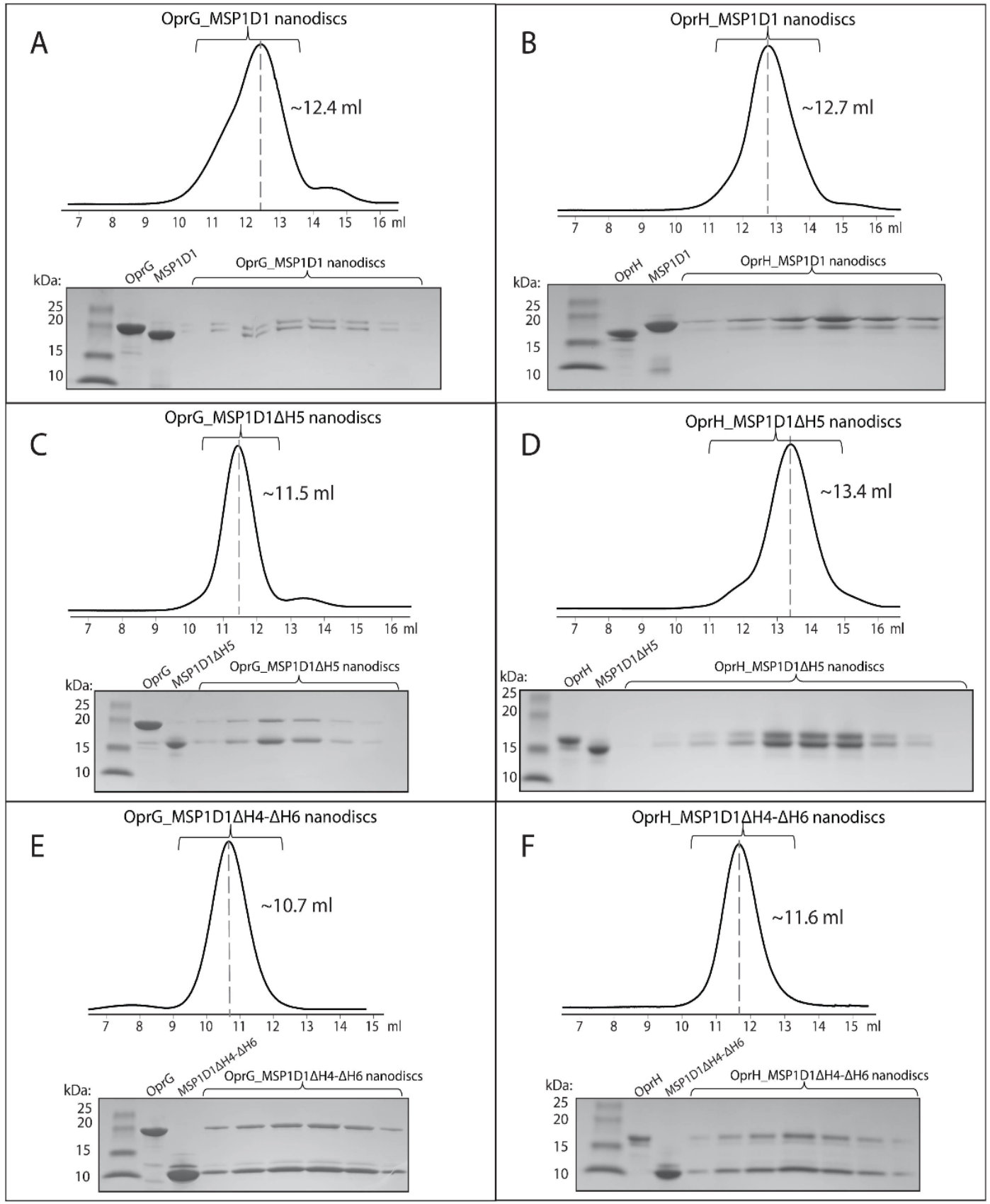

Figure 36. Purification of reconstituted OprG-MSP and OprH-MSP nanodiscs by size exclusion chromatography. A - OprG_MSP1D1, B - OprH_MSP1D1, C - OprG_MSP1D1 $\Delta \mathrm{H} 5$, D - OprH_MSP1D1 $\Delta \mathrm{H} 5, \quad$ E - OprG_MSP1D1 $\Delta \mathrm{H} 4-\Delta \mathrm{H} 6$, F OprH_MSP1D1 $\Delta \mathrm{H} 4-\Delta \mathrm{H} 6$. Each panel shows a chromatogram on top and an SDS-PAGE gel of the collected fractions (brackets) of the reconstituted membrane protein nanodiscs and the respective individual purified protein references. 
4.3.2 Assembly of Pseudomonas OM proteins in nanodiscs. We assembled OprG and OprH nanodiscs using the most stable and promising MSP constructs MSP1D1, MSP1D1 $\Delta H 4, M S P 1 D 1 \Delta H 5, M S P 1 D 1 \Delta H 4 \Delta H 5$ and MSP1D1 $\Delta H 4-\Delta H 6$. The designation $\Delta \mathrm{Hx}$ means that helix $\mathrm{x}$ has been deleted from the ten-helix segmented parent sequence MSP1D1. Assembled nanodiscs were purified by size exclusion chromatography and the resulting fractions were analyzed by SDS-PAGE (Fig. 36). The gel filtration elution profiles of the MSP1D1 $\Delta \mathrm{H} 4-\Delta \mathrm{H} 6$ nanodiscs (Fig. $36 \mathbf{E}$ and $\mathbf{F}$ ) indicate formation of larger assemblies, which has also been observed by Hagn et al [129] with these smallest nanodiscs containing the E. coli protein OmpX. Surprisingly, the size exclusion profile of OprG_MSP1D1 1 H5 (Fig. 36 C) also suggests that some nanodiscs may self-associate, but this behavior was not observed in case of OprH_MSP1D1 1 H5 nanodiscs (Fig. 36 D). Thus, the behavior of the introduced membrane protein matters on how a given species of

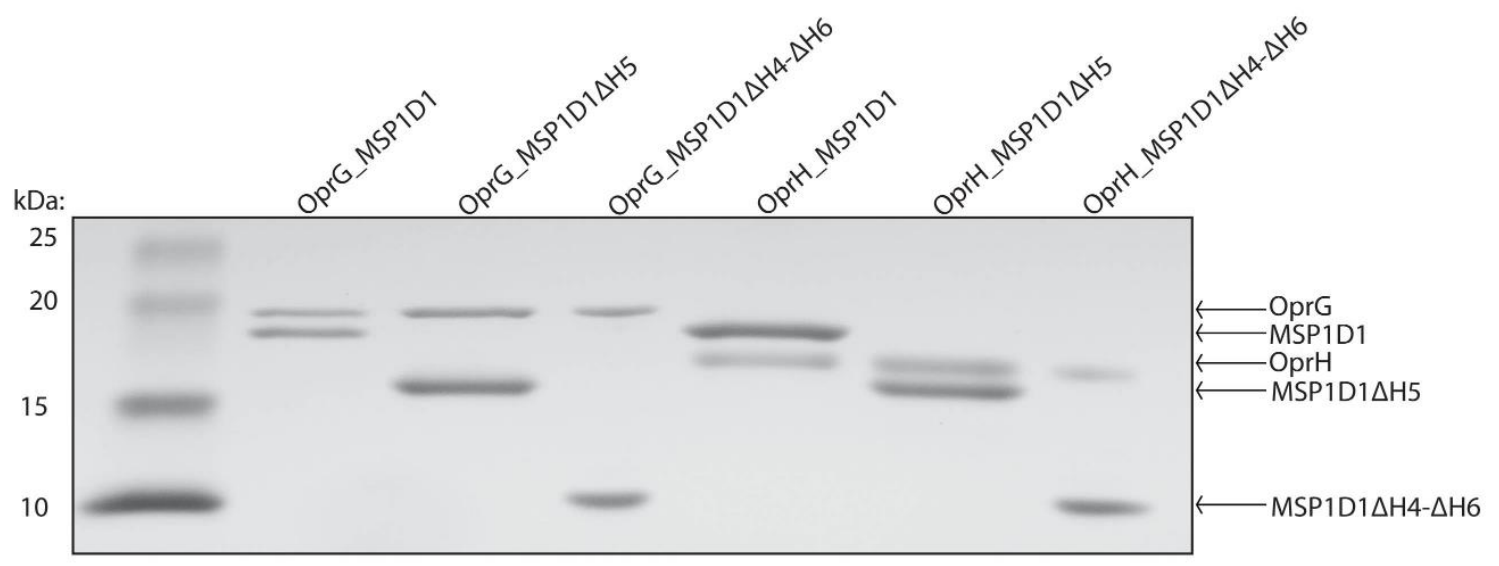

Figure 37. SDS-PAGE of final OprG-MSP and OprH-MSP nanodisc samples. Lane 1 protein standards, lane 2 - OprG_MSP1D1, lane 3 - OprG_ MSP1D1 $\Delta$ H5, lane 4 - OprG_ MSP1D1 $\Delta \mathrm{H} 4-\Delta \mathrm{H} 6$, lane $5-\mathrm{OprH} \_\mathrm{MSP} 1 \mathrm{D} 1$, lane $6-\mathrm{OprH}_{-} \mathrm{MSP} 1 \mathrm{D} 1 \Delta \mathrm{H} 5$, and lane 7 OprH_MSP1D1 $\Delta \mathrm{H} 4-\Delta \mathrm{H} 6$. 
nanodiscs behaves. In unrelated work we found that OprG has a tendency to oligomerize (unpublished results), i.e. a behavior that did not occur with OprH. Further optimization of the MSP:lipid ratios may alleviate some of these problems. An SDS-PAGE profile of the final reconstituted OprG and OprH nanodisc samples that were used for NMR experiments is shown in Fig. 37.

\subsubsection{NMR spectroscopy of OprG and OprH in nanodiscs of various sizes. After} extensive detergent screening with both OprG and OprH, DHPC proved to be optimal for the stability and NMR spectral quality of both proteins ([92] and unpublished results). Therefore, we use spectra of the proteins in DHPC as references to compare the spectral qualities of these proteins in nanodiscs and bicelles. The ${ }^{1} \mathrm{H}-{ }^{15} \mathrm{~N}-\mathrm{TROSY}$ spectrum of OprG in DHPC micelles (Fig. 38 A) shows good chemical shift dispersion with some peak overlaps in the ${ }^{1} \mathrm{H}$ dimension between 7.7-8.6 ppm (Fig. 38 A). For OprH in DHPC micelles the entire ${ }^{1} \mathrm{H}-{ }^{15} \mathrm{~N}$-TROSY spectrum is very well resolved (Fig. $39 \mathrm{~A}$ ) and, except for the flexible loop 1, could be almost completely assigned [92]. We collected ${ }^{1} \mathrm{H}-{ }^{15} \mathrm{~N}$-TROSY spectra of OprG and OprH containing nanodiscs assembled from DMPC and MSP1D1, MSP1D1 $\Delta H 4$, MSP1D1 $\Delta H 5, M S P 1 D 1 \Delta H 4 \Delta H 5$ and MSP1D1 $\Delta H 4-\Delta H 6$ (Figs. 38 B-F and 39 B-F, for OprG and OprH, respectively). We also assembled nanodiscs with uncut 6xHis-MSP proteins, but that resulted in nanodiscs with lower stability and reduced ${ }^{1}{ }^{H}-{ }^{15} \mathrm{~N}$-TROSY spectral quality for even those MSPs that behaved well with the cut His-tags (Fig. 40). In a few cases, we also assembled nanodiscs using a mixture of 


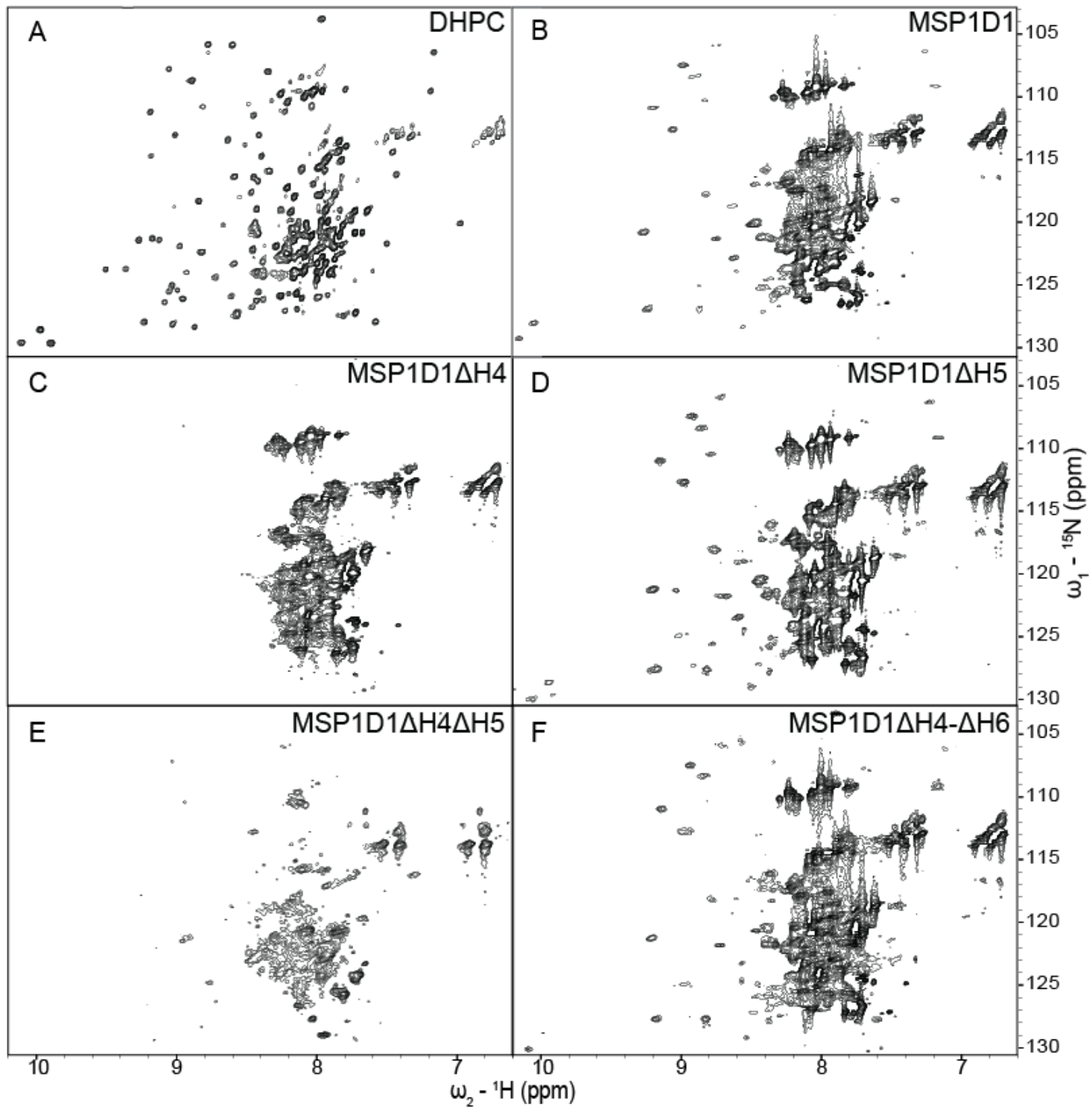

Figure 38. ${ }^{15} \mathrm{~N}-{ }^{1} \mathrm{H}$ TROSY spectra of ${ }^{2} \mathrm{H}-,{ }^{15} \mathrm{~N}$-labeled OprG in DHPC micelles and in MSP nanodiscs of different sizes. The OprG samples are in A - DHPC micelles, B - MSP1D1, C - MSP1D1 $\Delta \mathrm{H} 4, \mathbf{D}-\mathrm{MSP} 1 \mathrm{D} 1 \Delta \mathrm{H} 5, \mathrm{E}-\mathrm{MSP} 1 \mathrm{D} 1 \Delta \mathrm{H} 4 \Delta \mathrm{H} 5$, and F - MSP1D1 $\Delta \mathrm{H} 4-\Delta \mathrm{H} 6$. All spectra were collected at $800 \mathrm{MHz}$ and $45^{\circ} \mathrm{C}$.

DMPC and DMPG (1,2-dimyristoyl-sn-glycero-3-phospho-(1'-rac-glycerol)) at a ratio 3:1 (data not shown). We did not observe an improvement of the spectral quality over nanodiscs assembled with just DMPC. 


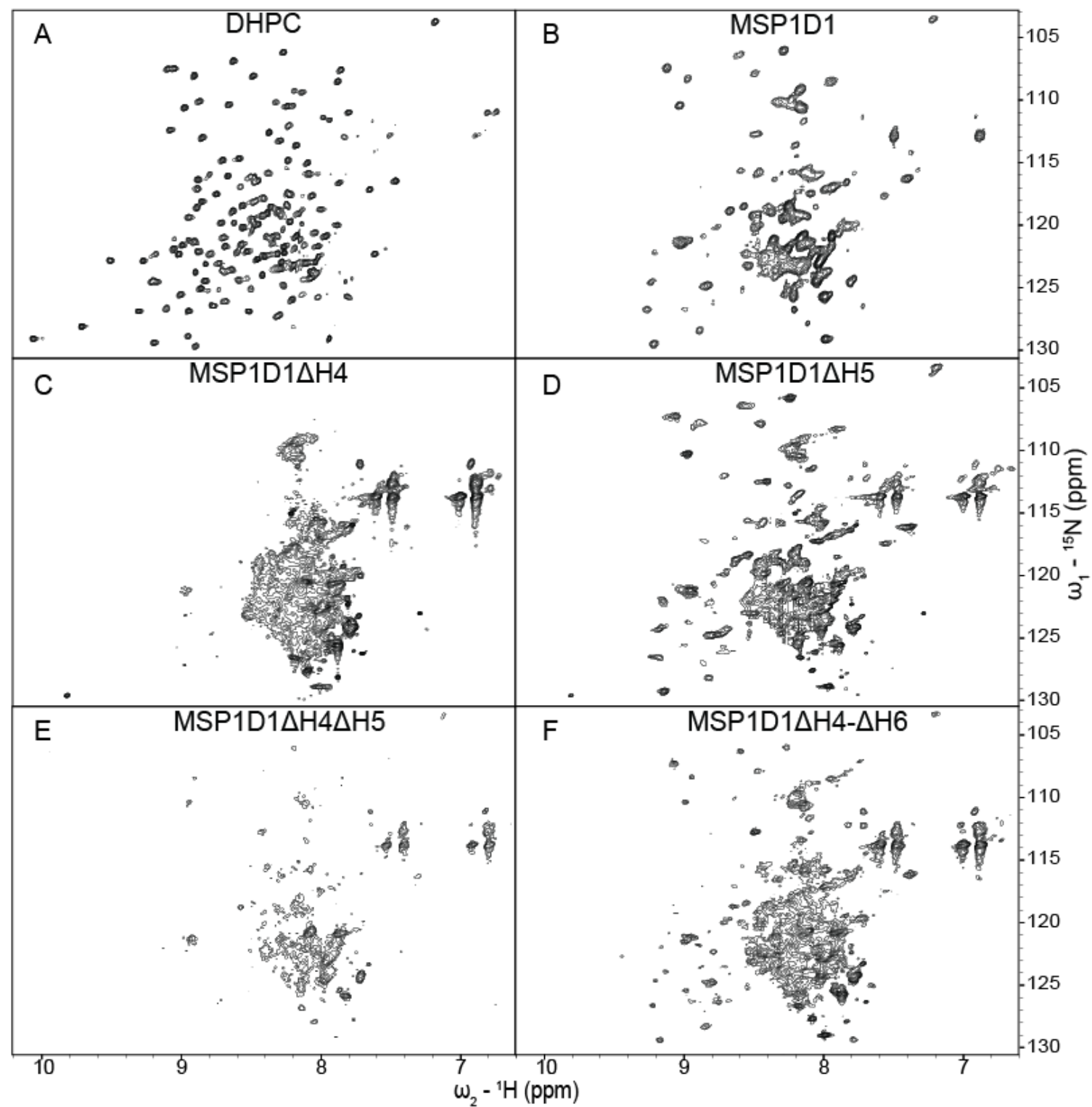

Figure 39. ${ }^{15} \mathrm{~N}-{ }^{1} \mathrm{H}$ TROSY spectra of ${ }^{2} \mathrm{H}-,{ }^{15} \mathrm{~N}$-labeled OprH in DHPC micelles and in MSP nanodiscs of different sizes. The OprH samples are in A - DHPC micelles, B - MSP1D1, C - MSP1D1 $\Delta \mathrm{H} 4, \mathrm{D}-\mathrm{MSP} 1 \mathrm{D} 1 \Delta \mathrm{H} 5, \mathrm{E}-\mathrm{MSP} 1 \mathrm{D} 1 \Delta \mathrm{H} 4 \Delta \mathrm{H} 5$, and F - MSP1D1 $\Delta \mathrm{H} 4-\Delta \mathrm{H} 6$. All spectra were collected at $800 \mathrm{MHz}$ and $45^{\circ} \mathrm{C}$.

Nanodiscs assembled from MSP1D1 $\Delta \mathrm{H} 4$ and MSP1D1 $\mathrm{H} 4 \Delta \mathrm{H} 5$ containing OprG or OprH proved to be unstable and partially precipitated during sample preparation and NMR data collection. ${ }^{1} \mathrm{H}^{15} \mathrm{~N}$-TROSY spectral qualities were intermediate for both $\mathrm{OM}$ 


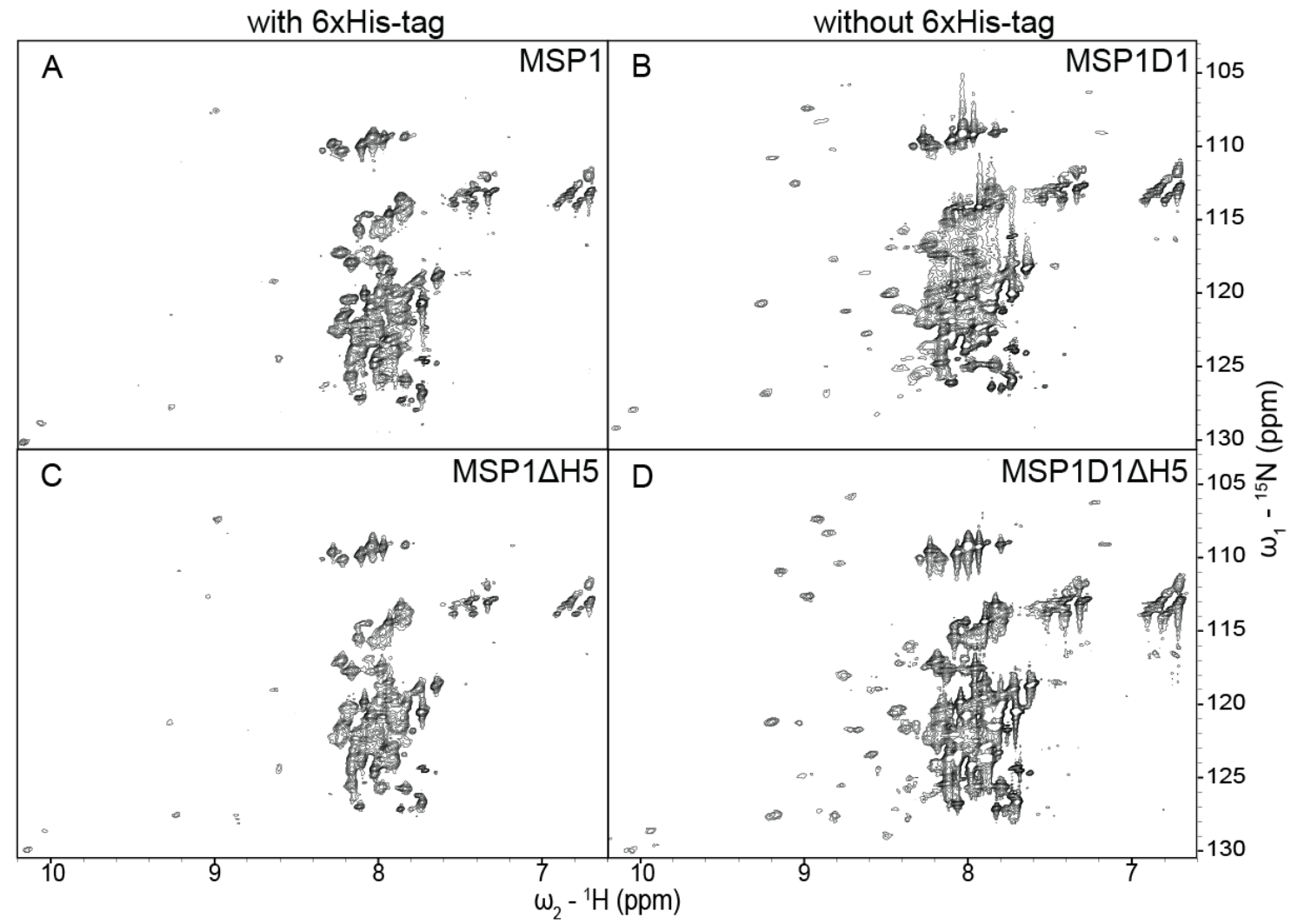

Figure 40. Comparison of ${ }^{15} \mathrm{~N}-{ }^{1} \mathrm{H}$ TROSY spectra of ${ }^{2} \mathrm{H}-,{ }^{15} \mathrm{~N}$-labeled OprG in MSP1 nanodiscs (with His-tag) or MSP1D1 nanodiscs (without His-tag). The following MSP constructs were used: A - HisMSP1, B - MSP1D1, C - HisMSP1 HH5, D - MSP1D1 H5. All spectra were collected at $800 \mathrm{MHz}$ and $45^{\circ} \mathrm{C}$.

proteins when they were reconstituted in the relatively large MSP1D1 parent nanodiscs

(Figs. $38 \mathrm{~B}$ and $39 \mathrm{~B}$ ). MSP1D1 $\Delta \mathrm{H} 5$ and MSP1D1 $\Delta \mathrm{H} 4-\Delta \mathrm{H} 6 \mathrm{DMPC}$ nanodiscs provided the best quality ${ }^{1} \mathrm{H}-{ }^{15} \mathrm{~N}$-TROSY spectra in the case of OprG (Fig. 38 D,F) and OprH (Fig. 39 D,F). However, even in the best quality nanodiscs the cross-peak intensities of the ${ }^{1} \mathrm{H}$ ${ }^{15} \mathrm{~N}-$ TROSY spectra were not homogenous and only $~ 70 \%$ of all cross-peaks that were observable in DHPC micelles could be detected (Table 4). Especially in case of OprG, broad cross-peaks in the 7.6-8.4 $\mathrm{ppm}^{1} \mathrm{H}$ region dominate over the weaker peaks in 8.4- 


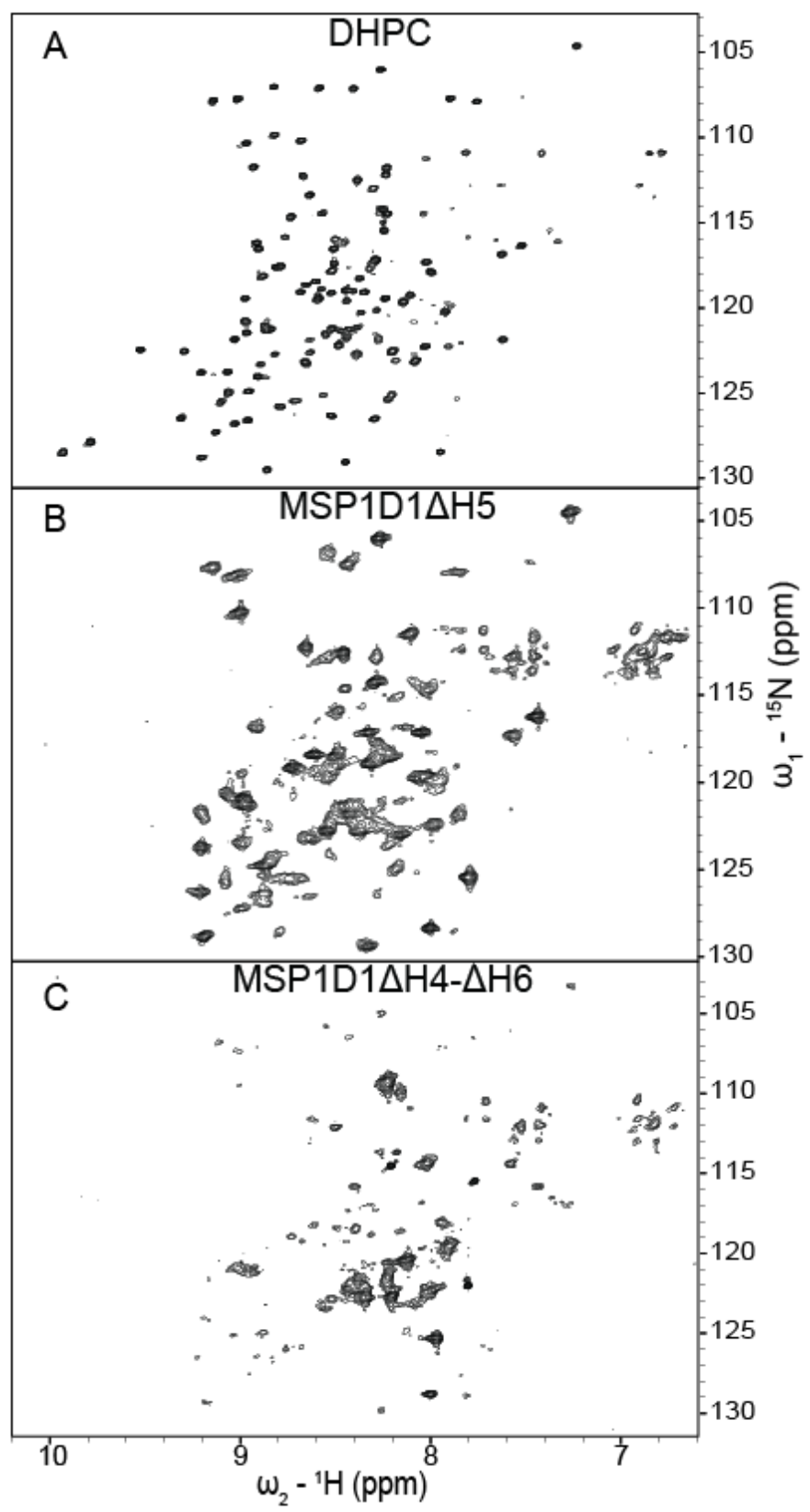

Figure 41. ${ }^{15} \mathrm{~N}-{ }^{1} \mathrm{H}$ TROSY spectra of ${ }^{2} \mathrm{H}-,{ }^{15} \mathrm{~N}$-labeled OprH $\Delta \mathrm{L} 1 \Delta \mathrm{L} 4$ in DHPC micelles and in MSP nanodiscs of different sizes. The OprH $\Delta \mathrm{L} 1 \Delta \mathrm{L} 4$ samples are in A - DHPC micelles, B MSP1D1 $\Delta \mathrm{H} 5$, and $\mathrm{C}-\mathrm{MSP} 1 \mathrm{D} 1 \Delta \mathrm{H} 4-\Delta \mathrm{H} 6$. All spectra were collected at $800 \mathrm{MHz}$ and $45^{\circ} \mathrm{C}$.

$9.4 \mathrm{ppm}{ }^{1} \mathrm{H}$ region. This behavior suggests that the protruding extracellular loops tumble independently from the well-ordered nanodisc-embedded $\beta$-barrel region of the 
Table 4. Number of resolved cross-peaks in the ordered regions of ${ }^{15} \mathrm{~N}-{ }^{1} \mathrm{H}$ TROSY spectra of the three proteins in DHPC micelles, $q=0.3$ PS-stabilized bicelles, and MSP nanodiscs of different sizes.

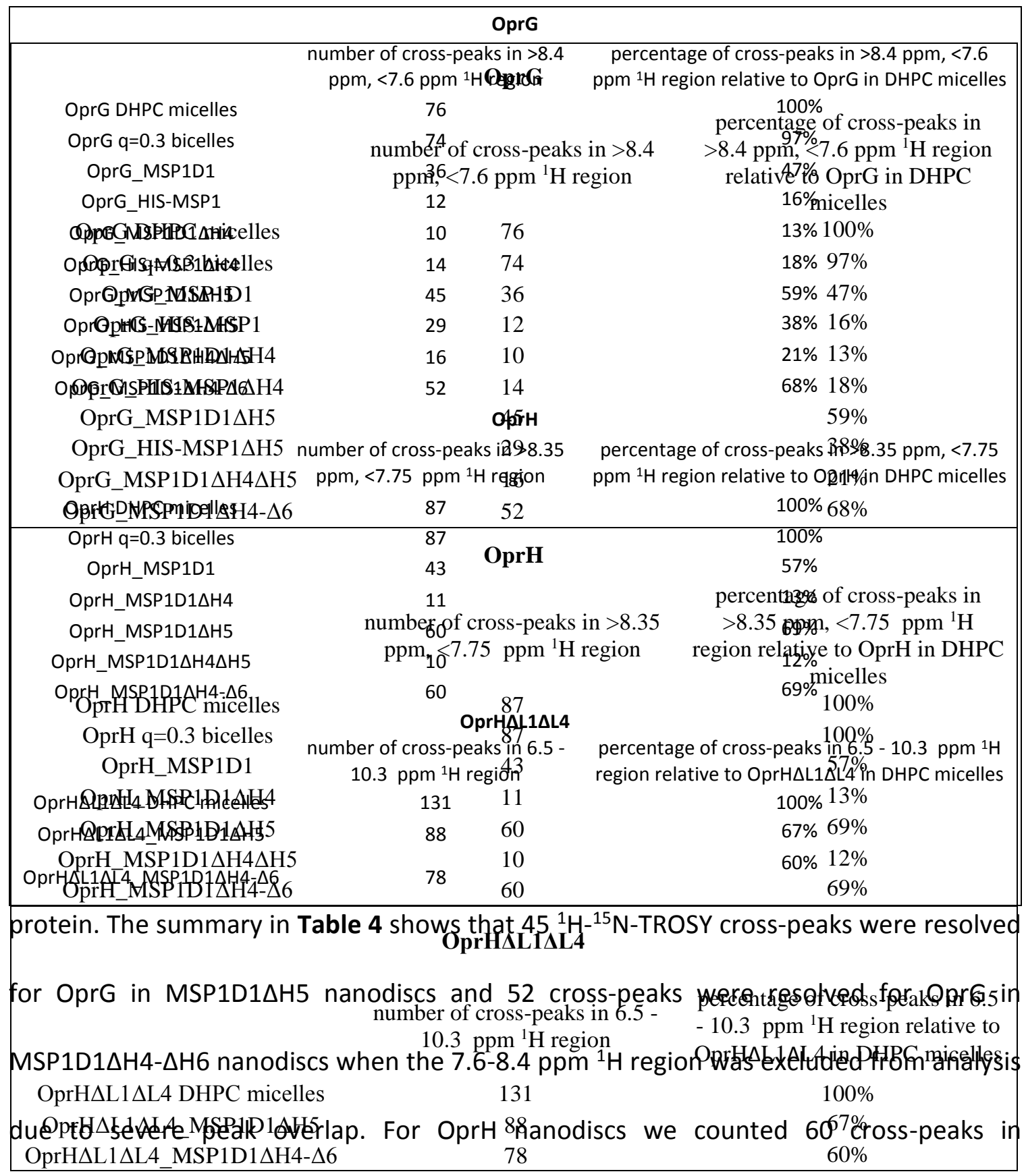

MSP1D1 $\Delta \mathrm{H} 5$ and in MSP1D1 $\Delta \mathrm{H} 4-\Delta \mathrm{H} 6$ nanodiscs when the $7.75-8.35 \mathrm{ppm}{ }^{1} \mathrm{H}$ region was

excluded. Even though MSP1D1 $\Delta \mathrm{H} 5$ nanodiscs provided better overall quality spectra 
than MSP1D1 $\Delta \mathrm{H} 4-\Delta \mathrm{H} 6$, several additional cross-peaks were identified in the OprG_MSP1D1 $\Delta \mathrm{H} 4-\Delta \mathrm{H} 6$ sample, which albeit weak could be mapped on the cross-peaks present in the spectrum of OprG in DHPC micelles.

Since the presence of the long unstructured loop regions of both proteins appears to be responsible for the observed inhomogeneous cross-peak intensities, we prepared an OprH variant, in which we removed the two longest loops. The loop 1 and loop 4 deletion mutant named $\mathrm{OprH} \Delta \mathrm{L} 1 \Delta \mathrm{L} 4$ showed ${ }^{1} \mathrm{H}-{ }^{15} \mathrm{~N}-\mathrm{TROSY}$ spectra of excellent quality in DHPC micelles (Fig. 41 A) and of good quality in MSP1D1 $\Delta$ H5 nanodiscs (Fig. 41 B), while its spectral quality in MSP1D1 $\Delta H 4-\Delta H 6$ nanodiscs was more mediocre (Fig. $41 \mathrm{C})$. Of the 131 cross-peaks present in the ${ }^{1} \mathrm{H}-{ }^{15} \mathrm{~N}-\mathrm{TROSY}$ spectra in DHPC micelles, 88 were present in MSP1D1 $\mathrm{H} 5$ nanodisc sample (Table 4). Further improvement to the quality of these spectra might be achieved by including deuterated instead of protonated DMPC in the nanodiscs. We tried to measure the apparent correlation time of OprH and OprG proteins in nanodiscs of various sizes by performing 1D TRACT experiment [119]. However, as OprH and OprG spectra are dominated by strong loop resonances, the obtained correlation time values did not reflect the correlation time of the tumbling particle. Only in case of $\mathrm{OprH} \Delta \mathrm{L} 1 \Delta \mathrm{L} 4$ in MSP1D1 $\Delta \mathrm{H} 5$ nanodiscs were we successful in measuring a correlation time of 32 ns (Fig. 42 A), which corresponds to a particle molecular mass of $\sim 100 \mathrm{kDa}$. 


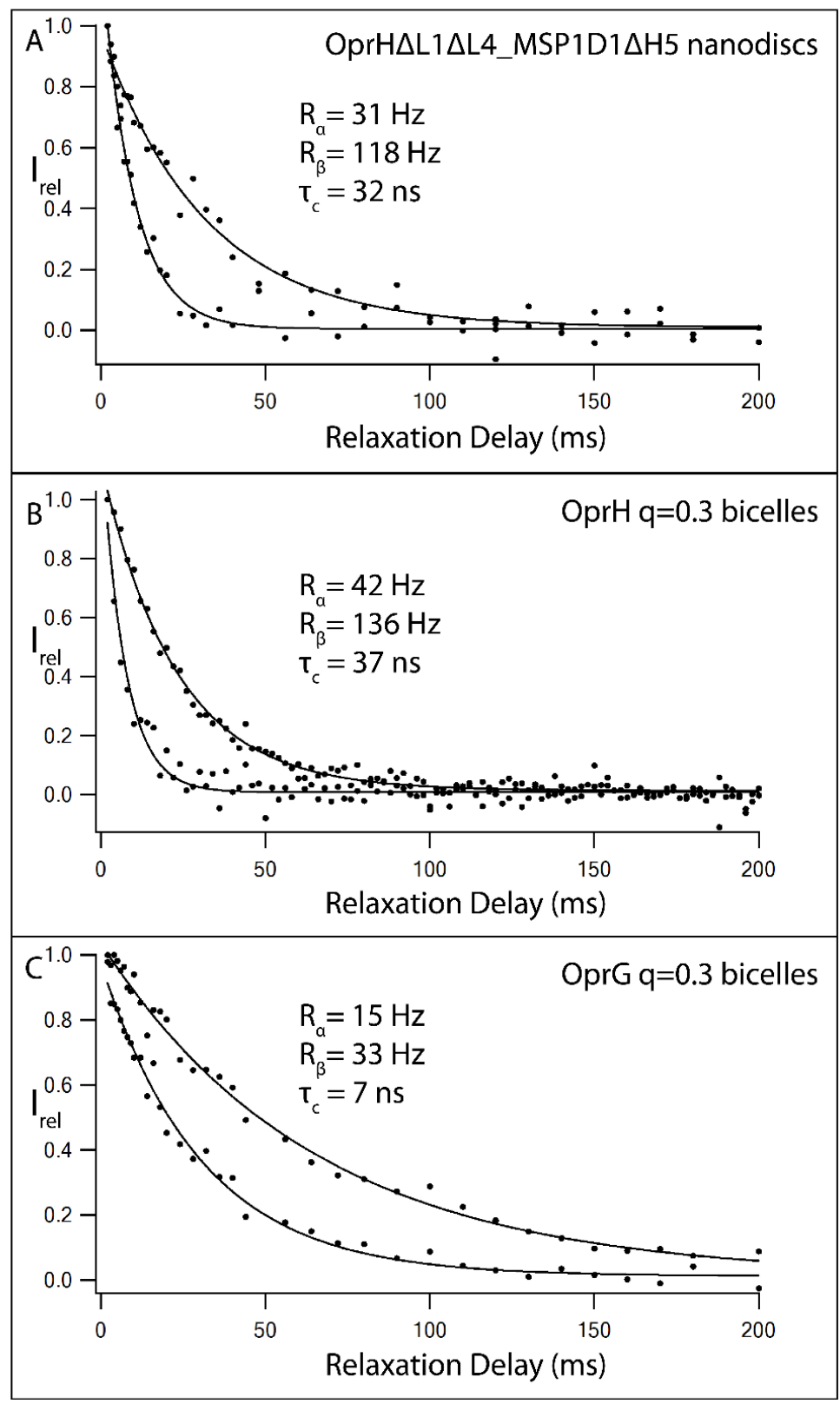

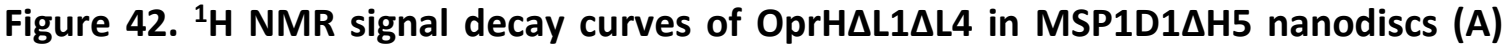
OprH in $q=0.3$ bicelles (B) and OprG in $q=0.3$ bicelles (C) acquired by 1D TRACT. The relative integral, $I_{\text {rel, }}$ was determined from integration of 1D spectrum over the region 6.5 - $10.5 \mathrm{ppm}$ for $\mathrm{OprH}$ and $\mathrm{OprH} \Delta \mathrm{L} 1 \Delta \mathrm{L} 4$ and 6.2 - $10.5 \mathrm{ppm}$ for OprG. The upper and lower curves correspond to the slow $\left(R_{\alpha}\right)$ and fast $\left(R_{\beta}\right)$ relaxing ${ }^{15} \mathrm{~N}$ spin-states, respectively. Values of $R_{\alpha}$ and $R_{\beta}$ were calculated by exponential curve fitting (solid line) and overall correlation times of $32 \mathrm{~ns}$ for OprH $\Delta \mathrm{L} 1 \Delta \mathrm{L} 4$ in MSP1D1 $\Delta \mathrm{H} 5$ nanodiscs, $37 \mathrm{~ns}$ for OprH in $\mathrm{q}=0.3$ bicelles and $7 \mathrm{~ns}$ for OprG in $\mathrm{q}=0.3$ bicelles were determined. Data were collected at $800 \mathrm{MHz}$ and $42^{\circ} \mathrm{C}$ for bicelles and $45^{\circ} \mathrm{C}$ for nanodiscs. 
4.3.4 NMR spectroscopy of OprG and OprH in lipid bicelles. Since lipid bicelles are also frequently used as alternatives to micelles in studies of membrane proteins by solution NMR, we wanted to compare the spectral qualities in bicelles with those of the same proteins in micelles and nanodiscs. Initial experiments of OprG and OprH in the most typical q=0.3 DHPC:DMPC bicelles indicated that these samples had tendency to aggregate over time. Therefore, we attempted to stabilize them by doping the bicelles with $10 \%$ of the equivalent chain-length negatively charged lipids DHPS and DMPS lipids leading to overall molar ratios of 27:9:3:1 of DHPC:DMPC:DHPS:DMPS. The rational for this approach was that the negatively charged particles would repel one another and thus prevent deleterious aggregation of the particles. Indeed, this approach allowed us to prepare high quality samples with both proteins that were stable over periods of several months.

To confirm the proper formation of OprH bicelles, we performed a 1D TRACT experiment (Fig. 42 B). The overall correlation time of the OprH in $\mathrm{q}=0.3$ PS-stabilized bicelles at $42^{\circ} \mathrm{C}$ was determined to be $37 \mathrm{~ns}$, which is significantly larger than $22 \mathrm{~ns}$ determined for OprH in DHPC micelles [92]. A correlation time of $37 \mathrm{~ns}$ corresponds to a protein-lipid bicelle complex of $100 \mathrm{kDa}$ with a composition of 1, 4, 12, 36 and 108 molecules of OprH, DMPS, DHPS, DMPC and DHPC, respectively. For comparison, OprH in DHPC micelles had an estimated molecular mass of 60-65 kDa [92]. A TRACT experiment performed with OprG bicelles was dominated by the flexible loops and 
produced an apparent correlation time of only $7 \mathrm{~ns}$ (Fig. $42 \mathrm{C}) .{ }^{1} \mathrm{H}-{ }^{15} \mathrm{~N}-\mathrm{TROSY}$ spectra of ${ }^{2} \mathrm{H},{ }^{13} \mathrm{C},{ }^{15} \mathrm{~N}$-labeled OprG and OprH PS-stabilized bicelle samples (Fig. 43) showed good chemical shift dispersion and exhibited approximately the same number of cross-peaks as their respective counterparts in DHPC micelles (Tab. 4). This result encouraged us to collect triple-resonance NMR experiments for a de novo assignment of $\mathrm{OprH}$ in $\mathrm{q}=0.3$ bicelles.

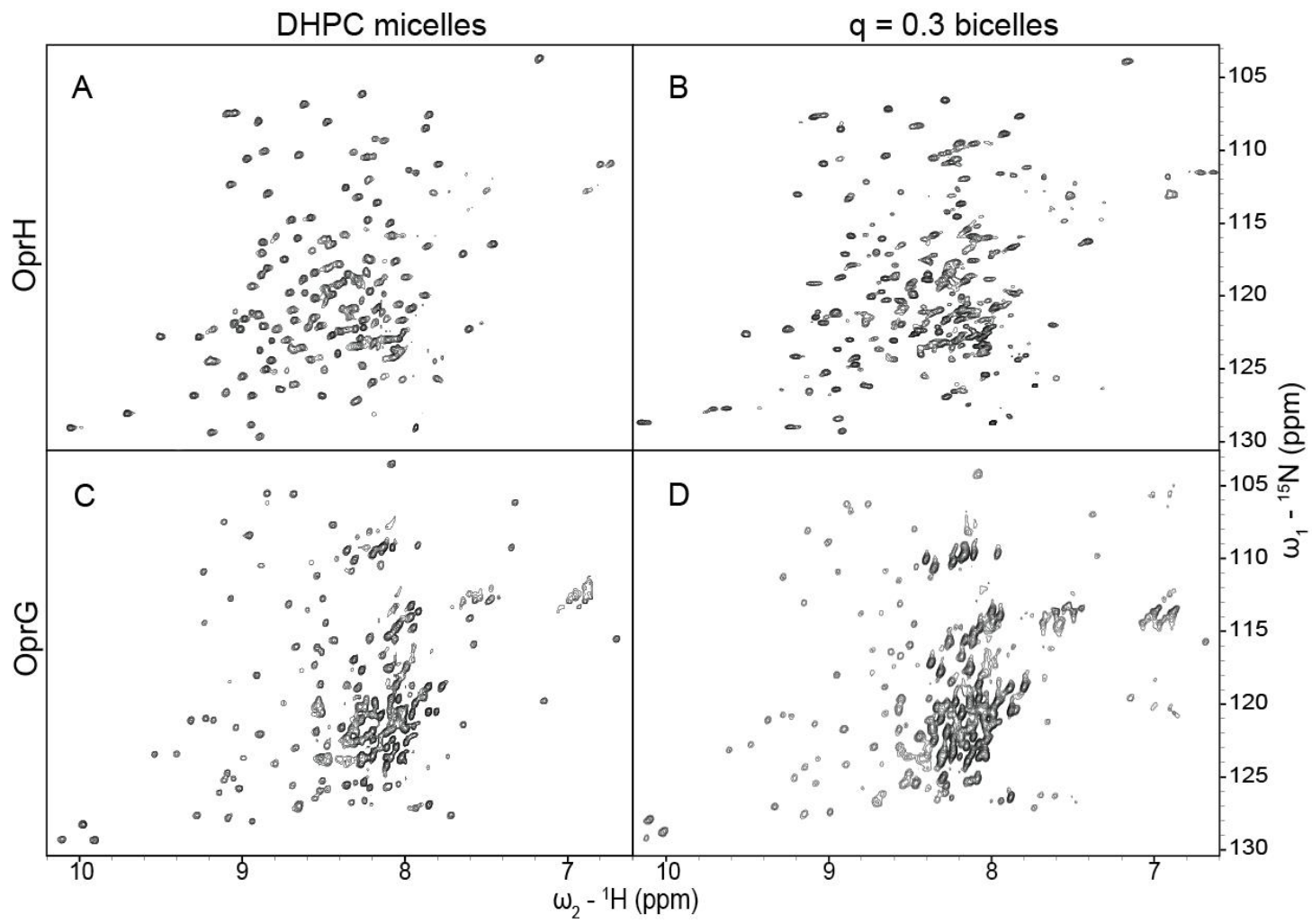

Figure 43. ${ }^{15} \mathrm{~N}-{ }^{1} \mathrm{H}$ TROSY spectra of ${ }^{2} \mathrm{H}-,{ }^{13} \mathrm{C}-,{ }^{15} \mathrm{~N}$-labeled OprH (A and B) and OprG (C and $D$ ) in DHPC micelles ( $A$ and $C$ ) and in $q=0.3$ DHPC:DMPC:DHPS:DMPS (27:9:3:1) bicelles (B and D). All spectra were collected at $800 \mathrm{MHz}$ and $45^{\circ} \mathrm{C}$.

TROSY versions of HNCA, HNCB, HNCO and HNCACO experiments were performed to assign $\mathrm{HN}, \mathrm{N}, \mathrm{C} \alpha, \mathrm{C} \beta$, and $\mathrm{CO}$ resonances of ${ }^{2} \mathrm{H},{ }^{13} \mathrm{C},{ }^{15} \mathrm{~N}-\mathrm{OprH}$ in $\mathrm{q}=0.3 \mathrm{PS}$ stabilized bicelles. 139 of 180 residues (excluding the lead methionine and 6xHis-tag) 
could be completely assigned which is only 17 residues fewer than the 156 residues assigned in DHPC micelles [92]. The resonances of these 17 residues were more broadened or their assignments were more ambiguous in the bicelle than in the micelle samples. Fig. 44 A shows the local secondary chemical shifts calculated from the C $\alpha$ and $\mathrm{C} \beta$ chemical shifts of OprH in bicelles [116]. The secondary chemical shifts of OprH are sensitive to the presence and type of secondary structure with large negative values indicating $\beta$-strands. There are eight distinct regions of large negative values characteristic of $\beta$-strands with shorter breaks of values around zero, typical for turns or random coils. The overall topology and the limits of well-defined $\beta$-structures of the protein were virtually the same for OprH in DHPC micelles and $q=0.3$ PS-stabilized bicelles (compare Fig. 44 A with Fig. 4 in [92]). Although longer chain lipids are present in the bicelles, this did not extend the lengths of the $\beta$-strands.

We also calculated the compound $\mathrm{NH}$ and $\mathrm{N}$ chemical shift differences $\left.\left(\Delta \delta_{\text {comp }}=\left[\Delta \delta^{2} \mathrm{HN}+\left(\Delta \delta_{\mathrm{N}} / 6.5\right)^{2}\right]\right]^{1 / 2}\right)[180]$ between ${ }^{2} \mathrm{H},{ }^{15} \mathrm{~N},{ }^{13} \mathrm{C}$-labeled OprH in micelles and $q=0.3$ bicelles (Fig. 44 B). Most residues showed no significant chemical shifts differences, but there were some notable exceptions. Significant differences were observed for residues $37,38,40$ and 42 on $\beta$-strand 2 , residues $136-138$ on $\beta$-strand 7 , residues $171,173,175$ and 176 on $\beta$-strand 8 , and residues 86 and 87 on periplasmic turn 2. Other minor chemical shift differences were localized in the neighborhood of the 
A

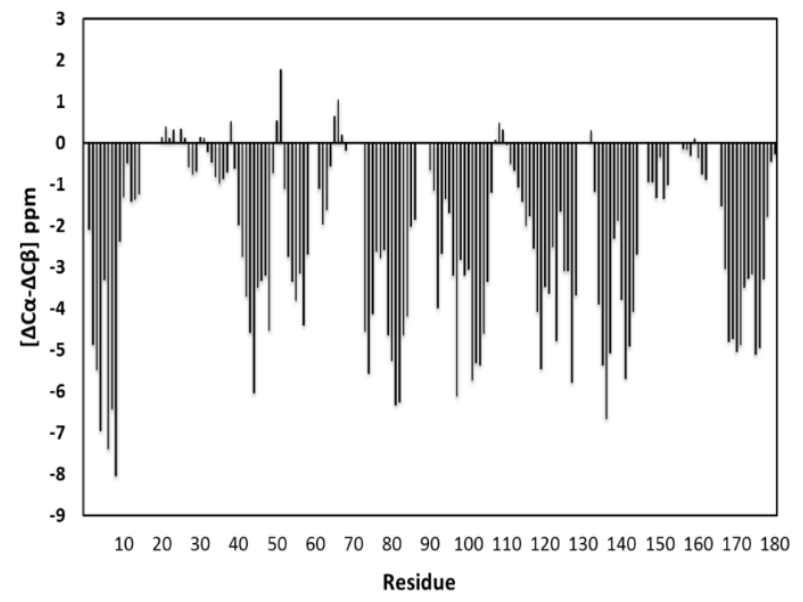

C

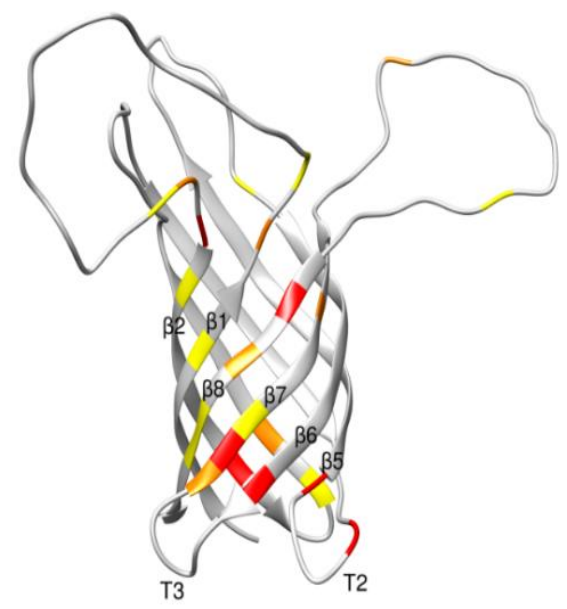

B
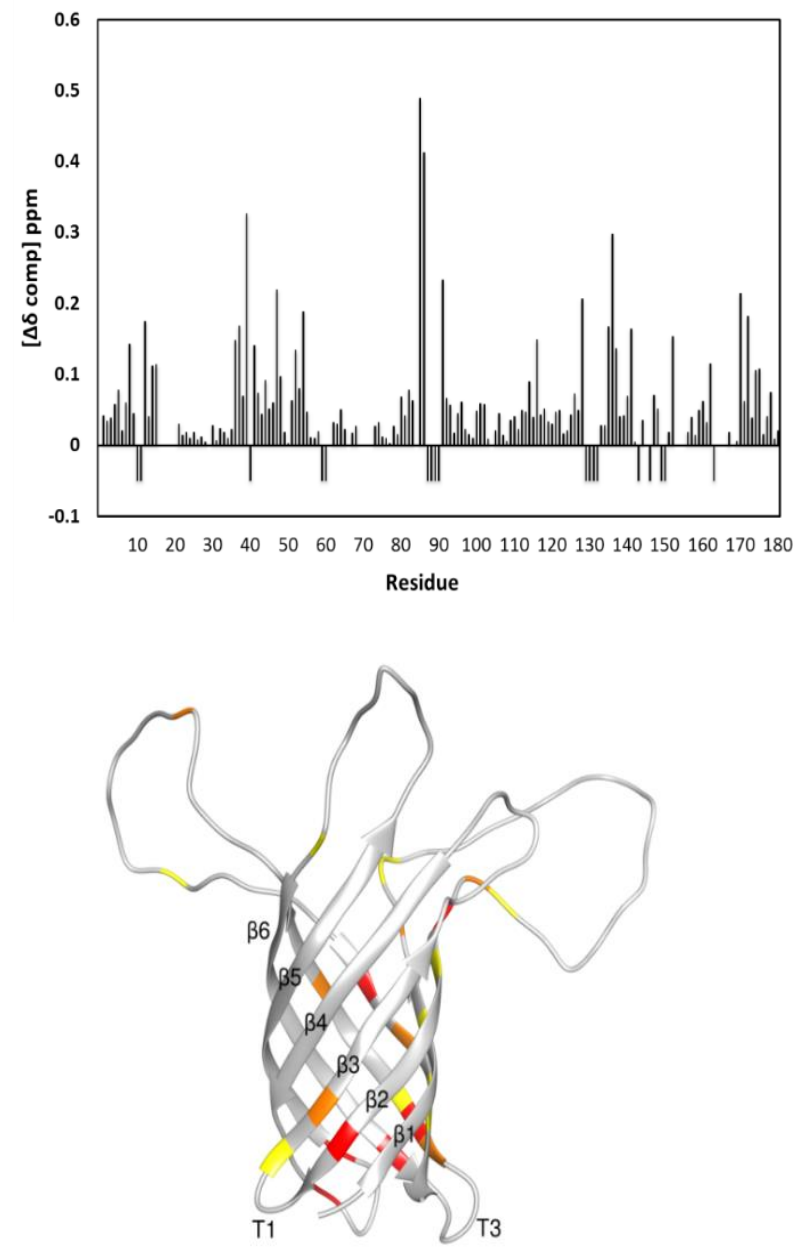

Figure 44. Chemical shift differences between OprH in DHPC micelles and $q=0.3$ DHPC:DMPC:DHPS:DMPS (27:9:3:1) bicelles. (A) Three-bond averaged ${ }^{13} \mathrm{C}$ secondary chemical shifts of ${ }^{2} \mathrm{H}-,{ }^{15} \mathrm{~N}-,{ }^{13} \mathrm{C}$-labeled OprH in q=0.3 DHPC:DMPC:DHPS:DMPS (27:9:3:1) bicelles. (B) Compound chemical shift differences $\left(\Delta \delta_{\text {comp }}=\left[\Delta \delta^{2}{ }_{H N}+\left(\Delta \delta_{N} / 6.5\right)^{2}\right]^{1 / 2}\right)[180]$ between ${ }^{2} \mathrm{H},{ }^{15} \mathrm{~N},{ }^{13} \mathrm{C}$-labeled $\mathrm{OprH}$ in micelles and $\mathrm{q}=0.3$ bicelles. Negative values indicate OprH residues unassigned in $\mathrm{q}=0.3$ bicelles. $(\mathrm{C})$ The compound chemical shift differences of (B) are mapped onto the lowest-energy conformer of OprH in DHPC micelles (PDB code: $2 \mathrm{LHF}$ ). Increasing chemical shift changes are depicted with increasingly more red colors. Yellow, orange, and red depict $\Delta \delta$ changes of $0.1-0.15,0.15-0.2$, and more than $0.2 \mathrm{ppm}$, respectively. The largest chemical shift perturbations occur near the interfacial regions of the $\beta$-strands and on the front face (left) rather than on the back face (right) of the $\beta$-barrel 
more significant ones and a very few minor changes occurred on some isolated extracellular loop residues. These chemical shift differences are mapped in Fig. $\mathbf{4 4 ~ C ~ i n ~}$ a graded color scheme onto the lowest-energy conformer of the previously determined structure of OprH [92]. From an inspection of this figure it is apparent that more changes occur on the front side (left panel) than on the back side (right panel) of the $\beta$-barrel and an abundance of changes are located near the two membrane-interfacial rims of the $\beta$ barrel.

\subsection{Discussion}

Structural studies of integral membrane proteins require a membrane-mimetic environment that supports their correct fold and activity. In this work we have compiled a detailed comparison of the quality of solution NMR spectra of two $\beta$-barrel membrane proteins, OprG and OprH from $P$. aeruginosa, in different lipidic environments: DHPC micelles, DHPC:DMPC:DHPS:DMPS bicelles and five different DMPC nanodiscs of varied sizes. Lipid micelles are most commonly used in solution NMR of integral membrane proteins. They form relatively small particles, typically on the order of 40-60 kDa for the most successfully used amphiphiles with membrane proteins on the order of 20-25 kDa that have yielded well-resolved solution NMR spectra. In many cases, including the case of OprH [92], structures of such micelle-embedded membrane protein samples could be solved. 
Small q ("isotropic") bicelles form particles that are substantially larger (80-120 kDa including a $~ 20-25$ kDa membrane protein) and therefore are more challenging for structural studies by solution NMR. The obvious advantage of bicelles is that they contain long-chain lipids such as DMPC that on their own form lipid bilayers and thus may provide a more natural environment for embedded membrane proteins. For the cases of OprG and OprH in the current study we estimate that about 40 long-chain lipids (DMPC and DMPS) were present in the sample for each molecule of protein, which is sufficient to surround the perimeters of these $\beta$-barrel proteins by a complete double layer of long-chain lipids. However, about 120 short-chain lipids per protein were also present in these samples. These short-chain lipids are most likely in rapid exchange with the long-chain lipids [158]. Therefore, the membrane proteins likely experience transient interactions not only with the long-chain, but also with the short-chain lipids and their structures may hence be influenced by both types of lipids.

Lipid nanodiscs most closely approach the situation of a membrane protein in a native phospholipid bilayer. They are detergent-free, contain different amounts (depending on their size) of bilayered long-chain lipids, and are delimited by a belt formed by two copies of an amphipathic membrane scaffold protein. The parent scaffold protein MSP1D1 contains 189 residues that are organized into 10 amphipathic $\alpha$-helical segments of variable lengths. MSP1D1 nanodiscs are about $10 \mathrm{~nm}$ in diameter and contain about 160 molecules of DMPC plus two copies of MSP1D1. Their molecular 
mass without an incorporated integral membrane protein is around $130 \mathrm{kDa}$ or around $160 \mathrm{kDa}$ when a $25 \mathrm{kDa}$ integral protein is included [129], making them significantly more challenging than "isotropic" bicelles for solution NMR studies of included membrane proteins. MSP1D1 $\Delta \mathrm{H} 5$ and MSP1D1 $\mathrm{H} 4$ nanodiscs contain about 100 and 90 molecules of DMPC, respectively, are about $8 \mathrm{~nm}$ wide, and have empty molecular masses of $\sim 90 \mathrm{kDa}$. The even smaller nanodiscs MSP1D1 $\Delta \mathrm{H} 4 \Delta \mathrm{H} 5$ and MSP1D1 $\Delta \mathrm{H} 4-\Delta \mathrm{H} 6$ contain about 40 and 20 molecules of DMPC, are about 7 and $6 \mathrm{~nm}$ wide, and have empty molecular masses of $\sim 70$ and $\sim 50 \mathrm{kDa}$, respectively. However, the very smallest MSP1D1 $\Delta \mathrm{H} 4-\Delta \mathrm{H} 6$ nanodiscs, when not hosting an integral membrane protein, were not stable over time and appeared to be in equilibrium with larger particles as also reported by Hagn et al. [129].

Different sizes of nanodiscs presented here influence the size of the host protein that can be accommodated. MSP1D1 $\Delta \mathrm{H} 4 \Delta \mathrm{H} 5$ and MSP1D1 $\Delta \mathrm{H} 4-\Delta \mathrm{H} 6$ might be most useful for membrane-anchored proteins and single-transmembrane helices, MSP1D1 $\Delta \mathrm{H} 5$ and MSP1D1 $\Delta \mathrm{H} 4$ can be used with proteins up to $\sim 30 \mathrm{kDa}$, for larger membrane proteins or membrane proteins multimers it is advised to use MSP1D1 or even larger MSP versions like MSP2 [129, 181].

Hagn et al. and Susak et al. performed detailed solution NMR studies on the 18 kDa membrane protein OmpX in MSP1D1 $\Delta \mathrm{H} 5$ and the $19 \mathrm{kDa}$ transmembrane domain of OmpA in MSP1D1 nanodiscs, respectively $[129,171]$. Since it is not a priori clear which 
MSP would be best for other membrane proteins, we screened the 23 and $21 \mathrm{kDa} \beta$ barrel membrane proteins OprG and OprH from Pseudomonas aeruginosa with five different MSPs. Nanodiscs formed from MSP1D1 $\triangle \mathrm{H} 5$ and DMPC clearly provided the best stability and best ${ }^{1} \mathrm{H}_{-}{ }^{15} \mathrm{~N}$ TROSY spectral quality for both OprG and OprH. Although this MSP has only one of the central 22-residue helical segments removed from the 189residue parent MSP1D1 protein, the diameter of MSP1D1 $\mathrm{H} 5$ nanodiscs is $1 \mathrm{~nm}$ less than that of MSP1D1 nanodiscs and the molecular mass of MSP1D1 $\Delta$ H5 nanodiscs is only about $70 \%$ that of MSP1D1. Based on the 1D TRACT result we obtained for $\operatorname{OprH} \Delta \mathrm{L} 1 \Delta \mathrm{L} 4$ in $\mathrm{MSP} 1 \mathrm{D} 1 \Delta \mathrm{H} 5$ nanodiscs, we estimate the molecular mass of OprH_MSP1D1 $\Delta \mathrm{H} 5$ nanodiscs to be around $105 \mathrm{kDa}$ and to contain about 70 molecules of DMPC arranged in a bilayer configuration around OprH. This number of lipids is sufficient to form a single continuous boundary lipid layer around the integrated membrane protein. Somewhat surprisingly, the very similarly sized nanodiscs formed from MSP1D1 $\triangle \mathrm{H} 4$ and DMPC were much less stable and performed much poorer in recorded ${ }^{1} \mathrm{H}-{ }^{15} \mathrm{~N}$ TROSY spectra with OprG and OprH. Recalling the similar behavior of OmpX in MSP1D1 $\mathrm{H} 4$ nanodiscs [129], it is apparent that these nanodiscs are, for unknown reasons, inferior to their equally sized sister MSP1D1 $\Delta \mathrm{H} 5$ nanodiscs. Hagn et al. also found MSP1D1 $\mathrm{H} 5$ to be the best scaffold protein for their spectroscopic studies of OmpX in nanodiscs [129]. The quality of their spectra was such that they were able to determine the structure of OmpX in MSP1D1 $\mathrm{H} 5$ nanodiscs. 
The second best MSP with OprG and OprH was MSP1D1 $\Delta \mathrm{H} 4-\Delta \mathrm{H} 6$, which had three 22-residue helical segments removed from MSP1D1 and which behaved more poorly with OmpX [129]. Although OprG and OprH reconstituted into the very small MSP1D1 $\Delta \mathrm{H} 4-\Delta \mathrm{H} 6$ nanodiscs showed initial promise, they had the unfortunate tendency to oligomerize in experiments requiring longer times for data collection. The second smallest nanodiscs MSP1D1 $\Delta \mathrm{H} 4 \Delta \mathrm{H} 5$ with two central 22-residue helical segments removed from the parent MSP1D1 scaffold protein performed poorly with all three OM proteins, OmpX, OprG, and OprH.

Even when the best nanodisc-forming MSP1D1 $\mathrm{H} 5$ was used, ${ }^{1} \mathrm{H}-{ }^{15} \mathrm{~N}$ TROSY spectra of OprG and OprH were characterized by broad linewidths and inhomogeneous peak intensities compared to spectra recorded in DHPC micelles. The broader linewidths are not surprising given the relatively large sizes of the nanodiscs. The inhomogeneous peak intensities can be explained by slow rotational correlation times of the nanodiscs and comparatively faster fluctuations of the long unstructured loops. Removing the two major loops from OprH in OprH $\Delta \mathrm{L} 1 \Delta \mathrm{L} 4$ yielded high quality ${ }^{1} \mathrm{H}_{-}{ }^{15} \mathrm{~N}$ TROSY spectra in MSP1D1 $\Delta \mathrm{H} 5$ nanodiscs, confirming our interpretation of the origin of the non-uniform peak intensities in the spectra of the full-length protein. It will likely be possible in future experiments to further improve on these spectra and perhaps perform comprehensive structural studies on OprH with the shortened loops by including deuterated lipids into the MSP1D1 $\Delta \mathrm{H} 5$ nanodiscs. 
The ${ }^{1} \mathrm{H}-{ }^{15} \mathrm{~N}$ TROSY spectra that we obtained in $\mathrm{q}=0.3$ DHPC:DMPC:DHPS:DMPS bicelles were of superior quality compared to the spectra obtained in any of the nanodiscs, but not quite as good as those obtained in micelles. This effect cannot be explained only by differences in size, as the OprH bicelles molecular mass calculated from the 1D TRACT experiment was very similar to the predicted molecular mass of OprH in MSP1D1 $\triangle \mathrm{H} 5$ nanodiscs. The reason for this difference may be that the protein rotates faster in bicelles than in nanodiscs. This is plausible considering that bicelles contain much more detergent than nanodiscs and that the detergent is likely in rapid dynamical exchange with the protein perimeter. We also did not observe any adverse effect of bicelles on OprG and OprH stability. Both proteins remained stable in DHPC:DMPC:DHPS:DMPS bicelles over the course of several months, as they did in DHPC micelles. These bicelles are therefore good membrane mimetics for solution NMR studies of both proteins. The extent of secondary structure was almost identical for OprH in micelles and bicelles, but some $\mathrm{HN}$ chemical shifts, especially those near the membrane interfaces on the front side of the protein were different for OprH in the two environments. Somewhat different networks of dynamic hydrogen-bonding may explain these relatively subtle differences. Since more of these changes occur on one face than on the other face of the barrel, it is possible that the protein has a tendency to (transiently) dimerize in one environment but not the other. However, this interpretation is currently speculative and will need further experimental testing, which is beyond the scope of the present work. 
In conclusion, it is clear that more extensive structural work by solution NMR is possible for two Pseudomonas $\beta$-barrel OM proteins in lipid bicelles and promising but challenging in some of the smaller nanodiscs. The protein $\operatorname{OprH} \Delta \mathrm{L} 1 \Delta \mathrm{L} 4$ with the shortened loops in MSP1D1 $\mathrm{H} 5$-DMPC nanodiscs is the most promising nanodisc sample at this stage. The quality of these spectra likely will be further improved in samples with deuterated lipids and by employing non-uniform NMR sampling methods [182-184]. This will make the collection of three-dimensional data sets and thorough structural studies of this protein in nanodiscs feasible. 


\section{Chapter 5. Summary and Conclusions}

In this dissertation I presented a detailed structure-function study through the use of spectroscopic, biochemical and microbiological techniques to elucidate the contributions of OMPs OprG and OprH to the physiology of $P$. aeruginosa.

To investigate the function and substrate specificity of OprG, I conducted comprehensive bacterial growth assays with a large set of potential substrates. These studies were complemented with liposome-swelling assays performed by a colleague in the laboratory. Despite previous reports suggesting that OprG might function as an iron, antibiotics or hydrophobic compound transporter, I discovered that it forms a channel for the transport and uptake of small amino acids including glycine, alanine, valine, serine, and threonine. We also verified the roles of three conserved proline residues that had been hypothesized by others to form a 'lateral gate' in the barrel wall. The mutation of Pro92 to an alanine resulted in a loss of the transport function, while mutations of Pro66 and Pro91 diminished the ability of the protein to insert into membranes. In order to obtain insight into the mechanistic and structural roles of these conserved prolines, I solved by NMR spectroscopy the dynamic structure of the P92A mutant of OprG and compared it with the dynamic structure of wild-type OprG in DHPC micelles. I found that the loops are dynamically disordered in the mutant and wild-type proteins, but that the height of the barrel wall and the loop dynamics are much more asymmetric in the mutant than in wild-type OprG. 
The function of OprH is to provide increased stability to the outer membranes of P. aeruginosa by directly interacting with LPS molecules. The NMR solution structure of OprH previously established in our lab revealed that this protein forms an eight-stranded $\beta$-barrel with four extracellular loops of unequal size. Chemical shift perturbations observed upon the addition of LPS to OprH in DHPC micelles indicate that the interaction is predominantly electrostatic and localized to loops 2 and 3 and charged regions near upper rim of the barrel. By applying ELISA with LPS adsorbed on the solid phase, I confirmed that the intact secondary structure is necessary for OprH to interact with LPS. Combining ELISA with site-directed mutagenesis allowed me to identify a set of positively charged residues localized in loops 2 and 3 (K70, R72, K103 and R113) that are essential for the interaction with LPS. Single mutations of these residues are not enough to abolish binding, but the triple mutant OprH-K70Q/R72Q/K103Q is no longer able to bind LPS. By monitoring the change in chemical shifts of ${ }^{15} \mathrm{~N}-{ }^{1} \mathrm{H}$-TROSY cross-peaks as a function of the LPS concentration I established the dissociation constant of binding of LPS to OprH to be $\sim 200 \mu \mathrm{M}$.

Solution NMR spectroscopy has become a robust method to determine structures and explore the dynamics and function of integral membrane proteins. The vast majority of studies on membrane proteins by solution NMR have been conducted in lipid micelles. Contrary to the lipids that form a lipid bilayer in biological membranes, micellar lipids typically contain only a single hydrocarbon chain or two chains that are too short to form a bilayer. Lipid bicelles and lipid nanodiscs have emerged as two alternative membrane 
mimetics that are compatible with solution NMR spectroscopy. To explore alternative, more bilayer-like media that mimic the natural environment of membrane proteins, I have conducted a comprehensive comparison of the physical and spectroscopic behavior of OprG and OprH, in lipid micelles, bicelles, and nanodiscs of five different sizes. Bicelles stabilized with a fraction of negatively charged lipids yielded spectra of almost comparable quality as observed in the best micellar solutions and the secondary structures were found to be almost indistinguishable in the two environments. Of the five nanodiscs tested, nanodiscs assembled from MSP1D1 $\Delta \mathrm{H} 5$ performed the best with both proteins in terms of sample stability and spectral resolution. Even in these optimal nanodiscs some broad signals from the membrane embedded barrel were severely overlapped with sharp signals from the flexible loops making their assignments difficult. A mutant form of OprH that had two of its flexible loops truncated yielded very promising spectra for further structural and dynamical analysis in MSP1D1 $\Delta \mathrm{H} 5$ nanodiscs.

\section{Future Directions}

Data presented here indicate that OprG functions as a channel for small amino acids. However, the precise mechanism of this transport remains to be elucidated. OprG forms a narrow pore, with both polar and apolar side chains facing the inside of the 8stranded $\beta$-barrel, which is probably too narrow to accommodate even the smallest transported amino acids. An alternative explanation that remains to be tested, is that OprG may form oligomers in membranes and that the relevant substrates may be 
translocated along a yet to be defined pore formed by multiple OprG units or OprG and the lipid bilayer. The fact that the mutant P92A is unable to facilitate amino acid transport in our liposome-swelling assay indicates that the unusual barrel wall in the region of the interfacial proline cluster may very well contribute to the mechanism of amino acid transport by OprG.

Our extensive characterization of the OprG and OprH proteins in lipid bicelles and nanodiscs indicates that further structural work by solution NMR is possible for these two Pseudomonas $\beta$-barrel OM proteins. The protein $\mathrm{OprH} \Delta \mathrm{L} 1 \Delta \mathrm{L} 4$ with the shortened loops in MSP1D1 $\triangle \mathrm{H} 5-\mathrm{DMPC}$ nanodiscs is the most promising nanodisc sample at this stage. The quality of these spectra likely will be further improved in samples with deuterated lipids and by employing non-uniform NMR sampling methods [182-184]. This should make the collection of well-resolved three-dimensional data sets and a thorough structural characterization of this protein in nanodiscs feasible. 


\section{Appendix 1. Investigation of the oligomerization}

\section{state of OprG in detergent micelles and lipid}

\section{bilayers}

\section{A1.1 Introduction}

This appendix summarizes unpublished data encompassing efforts to demonstrate the potential oligomerization state of OprG. Previous reports suggested that OprG is a monomer in the membranes $[73,74]$; however, the state of oligomerization of this protein has not been addressed biochemically. Additionally, the potential influence of the lipid environment on the oligomerization properties of OprG has not been investigated.

As mentioned in chapter 2.1 OprG forms an 8-stranded $\beta$-barrel with several polar and apolar side chains facing the inside. This channel is probably too narrow to accommodate any of the amino acids that we found can be translocated by OprG. Therefore I hypothesize that OprG may function as an oligomer in membranes and the amino acids might be transported along an interface between OprG monomers or between OprG and the lipid bilayer.

The oligomerization state of a protein may be deduced from its overall rotational correlation time. Rotational correlation times can be measured from ${ }^{1} \mathrm{H}-\mathrm{TRACT}$ NMR experiments and they were calculated to be 9 and 13 ns for wild-type and P92A OprG, 
respectively, when measured at $45^{\circ} \mathrm{C}$ in the $6.5-10.5 \mathrm{ppm}$ regions (Fig. 21). Compared to other membrane proteins of similar size, the apparent rotational correlation time of OprG is very low, which is probably the result of the relatively long disordered loops that tumble faster than the micelle-embedded barrel portions of the protein and therefore dominate the NMR signal.

To obtain more information about oligomeric state of OprG, I performed native PAGE and self-quenching experiments in detergent and in POPC liposomes. My results suggest that OprG forms oligomers in detergents and in lipid bilayers, however, additional experiments are required to establish the exact stoichiometry of these oligomers.

\section{A1.2 Methods}

A1.2.1 Site-Directed Mutagenesis. Cysteine mutations for Alexa-647 labeling were introduced to wild-type and P92A OprG at positions 116 or 166 . Mutagenesis was performed as described in section 2.2.2.

A1.2.2 Expression. Expression and purification of all proteins used was performed as described in section 2.2.1.

A1.2.3 Fluorophore Labeling. After elution from the NiNta column (as described in section 2.2.1.), purified OprG and P92A V116C and V166C mutants were incubated with a 10-fold molar excess of Alexa647 malemide (Invitrogen) overnight at room temperature. Free dye was removed by desalting on a PD-10 column (GE Healthcare). Typically, labeling efficiencies of $85-95 \%$ were achieved as determined by MALDI-TOF spectroscopy and absorbance measurements using the manufacturer's extinction 
coefficients. Labeled proteins were subsequently refolded in DHPC detergent as described in 2.2.3.

A1.2.4 Fluorescence Spectroscopy. All fluorescence experiments were performed at $25^{\circ} \mathrm{C}$ using a Fluorolog-3 spectrofluorometer (HORIBA Jobin-Yvon) following the method of [185]. Self-quenching experiments with Alexa647-labeled proteins were carried out with the excitation wavelength set at $650 \mathrm{~nm}$ and emission scans between 655 and $750 \mathrm{~nm}$. Five nanometer slits were used in the excitation and emission paths in all experiments. Peak fluorescence emission intensities were evaluated in all experiments. The results of at least three independent experiments were averaged in all experiments. Error bars represent single standard deviations.

A1.2.5 Preparation of Unilamellar Vesicles. Unilamellar vesicles were prepared by mixing appropriate amounts of stock lipid solutions in chloroform in a glass test tube. Chloroform was evaporated using a stream of nitrogen gas while rotating the test tube. The lipid film was further dried under vacuum for 4-8 hrs and hydrated with 20 mM HEPES $\mathrm{pH} 7.4,150 \mathrm{mM} \mathrm{NaCl}, 25 \mathrm{mM}$ sodium cholate. Appropriate amounts of protein in buffer containing $0.7 \%$ DHPC $(\mathrm{w} / \mathrm{v})$ were added and incubated for $1 \mathrm{~h}$ to solubilize the lipids and to form mixed protein/lipid/detergent micelles. Samples were diluted 3-fold and dialyzed extensively with $\sim 1 \mathrm{~g} / \mathrm{L} \mathrm{SM-2} \mathrm{BioBeads} \mathrm{(Bio-Rad).} \mathrm{Liposomes} \mathrm{were} \mathrm{centrifuged} \mathrm{at} 13200$ rpm for 60 min to remove any precipitated protein.

A1.2.6 CBQCA assay. The efficiency of protein reconstitution in the liposomes was measured using the CBQCA Protein Quantitation Kit (Thermo Fisher Scientific) following 
the method of the manufacturer. Before measurements the liposomes were centrifuged at $13200 \mathrm{rpm}$ for $60 \mathrm{~min}$ to remove any precipitated protein. Subsequently, sample volumes were adjusted to $135 \mu$ land liposomes were lysed with $0.1 \%$ Triton-X-100. $5 \mu \mathrm{L}$ of $20 \mathrm{mM} \mathrm{KCN}$ was added to each sample and mixed. After addition of $10 \mu \mathrm{L}$ of $5 \mathrm{mM}$ ATTO-TAG CBQCA in DMSO, samples were incubated at room temperature with shaking for 1 hour. The fluorescence was measured with the emission and excitation wavelengths set at $\sim 465 \mathrm{~nm}$ and $\sim 550 \mathrm{~nm}$, respectively. A standard curve was generated with BSA as a reference for converting the observed fluorescence to the mass of protein in the sample.

A1.2.7 Native PAGE. Native polyacrylamide gel electrophoresis was performed to analyze the oligomeric nature of the proteins in detergent and in liposomes. Liposome samples were partially solubilized in DDM detergent at 10:1 or 50:1 DDM:protein ratio before loading on the native gel. The protein samples were mixed with native loading dye (50 mM Tris-Cl pH 6.8, 0.5\% bromophenol blue, 5\% glycerol) and were loaded onto a 416\% gradient non-denaturing Native PAGE Bis-Tris gel (Novex). The cathode buffer (15 $\mathrm{mM}$ Tris- $\mathrm{Cl} \mathrm{pH} \mathrm{7.0,50} \mathrm{mM} \mathrm{Tricine)} \mathrm{contained} \mathrm{0.01 \%} \mathrm{Coomassie} \mathrm{Brilliant} \mathrm{Blue} \mathrm{G-250} \mathrm{and}$ the anode buffer was composed of $12.5 \mathrm{mM}$ Tris- $\mathrm{Cl} \mathrm{pH} 8.3,96 \mathrm{mM}$ glycine. The gel was run at $4^{\circ} \mathrm{C}$ and destained using $10 \%$ acetic acid in water.

\section{A1.3 Results}

A1.3.1 Protein reconstitution in liposomes. In order to find a lipid mixture that provides the highest protein reconstitution efficiency, I investigated the insertion of OprG 
into lipid bilayers of different composition (pure POPC, POPG:POPC 20:80, POPG:POPC 40:60, DMPC, Table A1.1). Protein stock concentrations in DHPC detergent and after

Table A1.1. Reconstitution efficiency of wt and P92A OprG into lipid bilayers of different lipid composition and different lipid:protein ratio, established using CBQCA assay.

\begin{tabular}{|c|c|c|c|c|}
\hline lipid & protein & lipid:protein ratio & $\begin{array}{c}\text { reconstitution } \\
\text { efficiency \% }\end{array}$ & $\begin{array}{l}\text { protein molecules } \\
\text { per liposome* }\end{array}$ \\
\hline \multirow{6}{*}{ POPC } & \multirow{3}{*}{ OprG } & 1000:1 & 38.2 & $6-10$ \\
\hline & & $500: 1$ & 36.7 & $12-18$ \\
\hline & & $250: 1$ & 38.5 & $25-39$ \\
\hline & \multirow{3}{*}{ P92A } & 1000:1 & 26.0 & $4-7$ \\
\hline & & 500:1 & 22.2 & $7-11$ \\
\hline & & $250: 1$ & 15.7 & $10-16$ \\
\hline \multirow{6}{*}{ DMPC } & \multirow{3}{*}{ OprG } & 1000:1 & 21.9 & $3-5$ \\
\hline & & $500: 1$ & 20.4 & $7-10$ \\
\hline & & $250: 1$ & 22.5 & $14-22$ \\
\hline & \multirow{3}{*}{ P92A } & 1000:1 & 6.8 & $1-2$ \\
\hline & & $500: 1$ & 6.9 & $2-3$ \\
\hline & & $250: 1$ & 5.9 & $4-6$ \\
\hline \multirow{3}{*}{ POPG $20 \%$} & \multirow{3}{*}{ OprG } & 1000:1 & 38.0 & $6-9$ \\
\hline & & $500: 1$ & 39.0 & $12-20$ \\
\hline & & 250:1 & 21.5 & $14-22$ \\
\hline \multirow[t]{3}{*}{ POPC $80 \%$} & \multirow{3}{*}{ P92A } & 1000:1 & 28.4 & $5-7$ \\
\hline & & $500: 1$ & 25.8 & $8-13$ \\
\hline & & $250: 1$ & 19.8 & $13-20$ \\
\hline \multirow{3}{*}{ POPG $40 \%$} & \multirow{3}{*}{ OprG } & 1000:1 & 38.7 & $6-10$ \\
\hline & & $500: 1$ & 37.3 & $12-19$ \\
\hline & & $250: 1$ & 20.8 & $13-21$ \\
\hline \multirow[t]{3}{*}{ POPC $60 \%$} & \multirow{3}{*}{ P92A } & $1000: 1$ & 29.9 & $5-7$ \\
\hline & & $500: 1$ & 22.3 & $7-11$ \\
\hline & & $250: 1$ & 19.2 & $12-19$ \\
\hline
\end{tabular}

*assuming vesicle size of $40-50 \mathrm{~nm}$

reconstitution into liposomes were determined using CBQCA assay (Thermo Fisher Scientific), as it provides good accuracy of protein concentrations in samples containing high concentrations of lipids and detergents. Despite multiple efforts, I was unable to successfully reconstitute OprGP66A and OprGP91A in any lipid mixture that I tested. Wt 
OprG and P92A OprG showed different efficiencies of reconstitution depending on the lipid mixture and protein:lipid ratio used. In general, pure POPC and POPC mixtures performed better than pure DMPC. Wt OprG also incorporated better into lipid bilayers than P92A OprG. For example, P92A OprG showed only 6-7\% reconstitution efficiency in pure DMPC and 20-30\% efficiency in POPG:POPC 40:60 lipid mixture, while wt OprG showed $\sim 20 \%$ and up to $\sim 40 \%$ in these lipid mixtures.

A1.3.2 Native Page. To determine the oligomeric state of wt OprG and its single proline mutants in detergent and liposomes I performed native PAGE analysis. Wt P92A, P66A, P91A OprG samples in DHPC (Fig. A1.1 A) showed almost identical patterns on the native gels, with multiple bands indicating the presence of monomers, dimers and larger

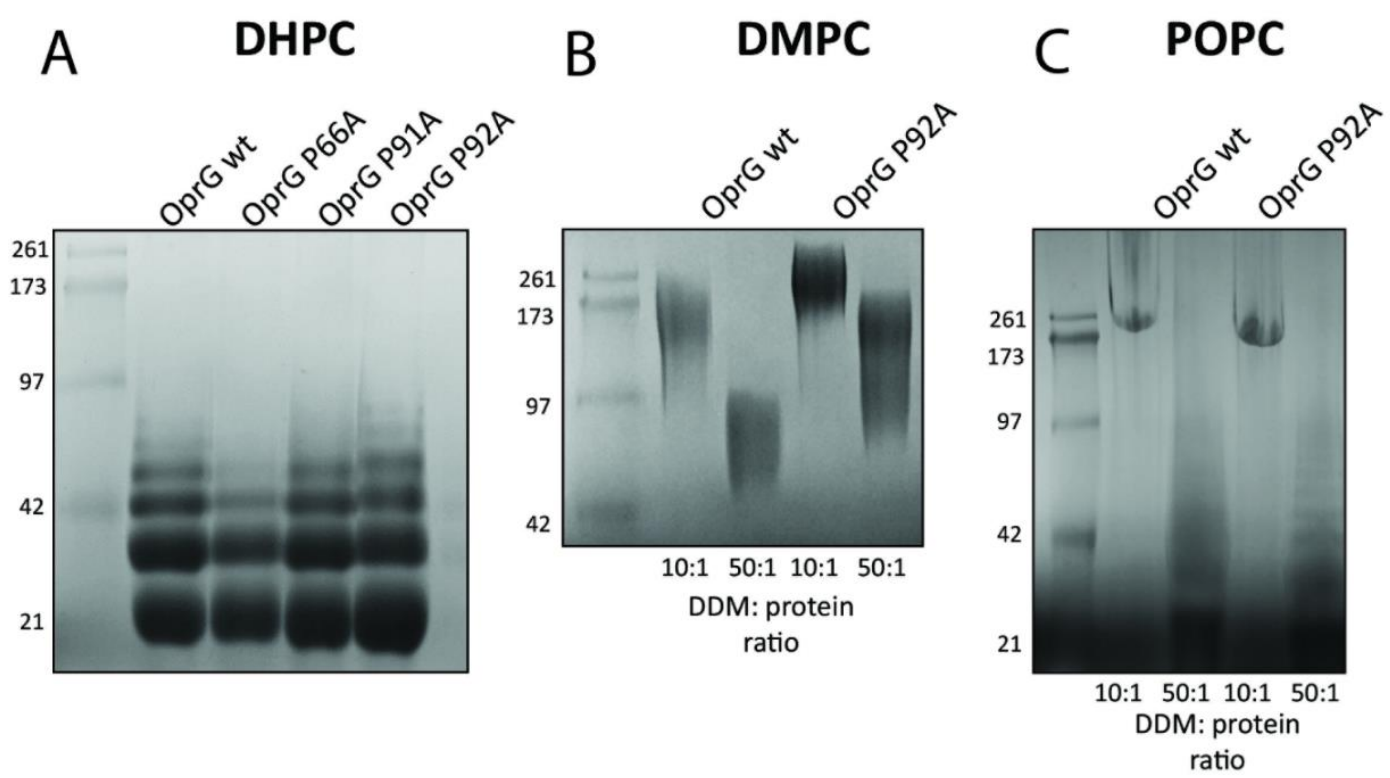

Fig A1.1. Native PAGE of OprG samples in DHPC detergent (A), and in DMPC (B) and POPC (C) liposomes. DMPC and POPC liposomes were partially solubilized with DDM detergent at ratios indicated at the bottom of the gels. 
species. In order to analyze wt and P92A OprG in DMPC (Fig. A1.1 B) and POPC (Fig. A1.1 C) liposome samples, I partially solubilized them in DDM at 10:1 and 50:1 DDM:protein ratios. These samples revealed the presence of high mass molecular protein or proteinlipid aggregates on the order of 100-200 kDa. At 50:1 DDM:protein ratio, these aggregates break down partially, especially in the case of the POPC samples and to some extent in the case of the wt OprG/DMPC sample.

A1.3.3 Fluorescence quenching measurements. In order to investigate the multimerization of OprG in detergent micelles and liposomes, I fluorescently labeled the wt and P92A OprG proteins at position 116 or 166 with Alexa647. Valine 116 and 166 were selected as the labeling sites, as according to our structure (2.3.4), they are localized on the outside just above the barrel rim, which should ensure reasonable exposure to water without introducing too high flexibility. Both residues were mutated to cysteines prior to labeling. Since the labeling reaction with Alexa647 was performed on the unfolded form of the protein in $8 \mathrm{M}$ urea, selected sites were fully accessible to the reagent and the labeling efficiencies were $85-95 \%$.

Fig. A1.2 A shows the dependence of the fluorescence of free Alexa 647 molecules on the Alexa647 concentration in DHPC micelles. As the concentration decreases from 26 to $13 \mu \mathrm{M}$, the fluorescence intensity of free Alexa 647 increases $\sim 2.9$ fold. This inverse concentration behavior of the fluorescence intensity most likely reflects self-quenching of Alexa647 at increasing concentrations. In the case of A647-labeled OprG in DHPC micelles (Fig. A1.2 B), the fluorescence intensity decreased approximately 2.9 fold as the 
concentration of OprG increased from 10 to $25 \mu \mathrm{M}$, which is almost identical to the behavior of the free dye in the same concentration range. This suggests that the decrease of the wt OprG fluorescence is simply due to the effect of increasing concentration. However, in case of P92A, the fluorescence decreased 3.6 fold over the same concentration range, which suggest some degree of association of individual P92A monomers.
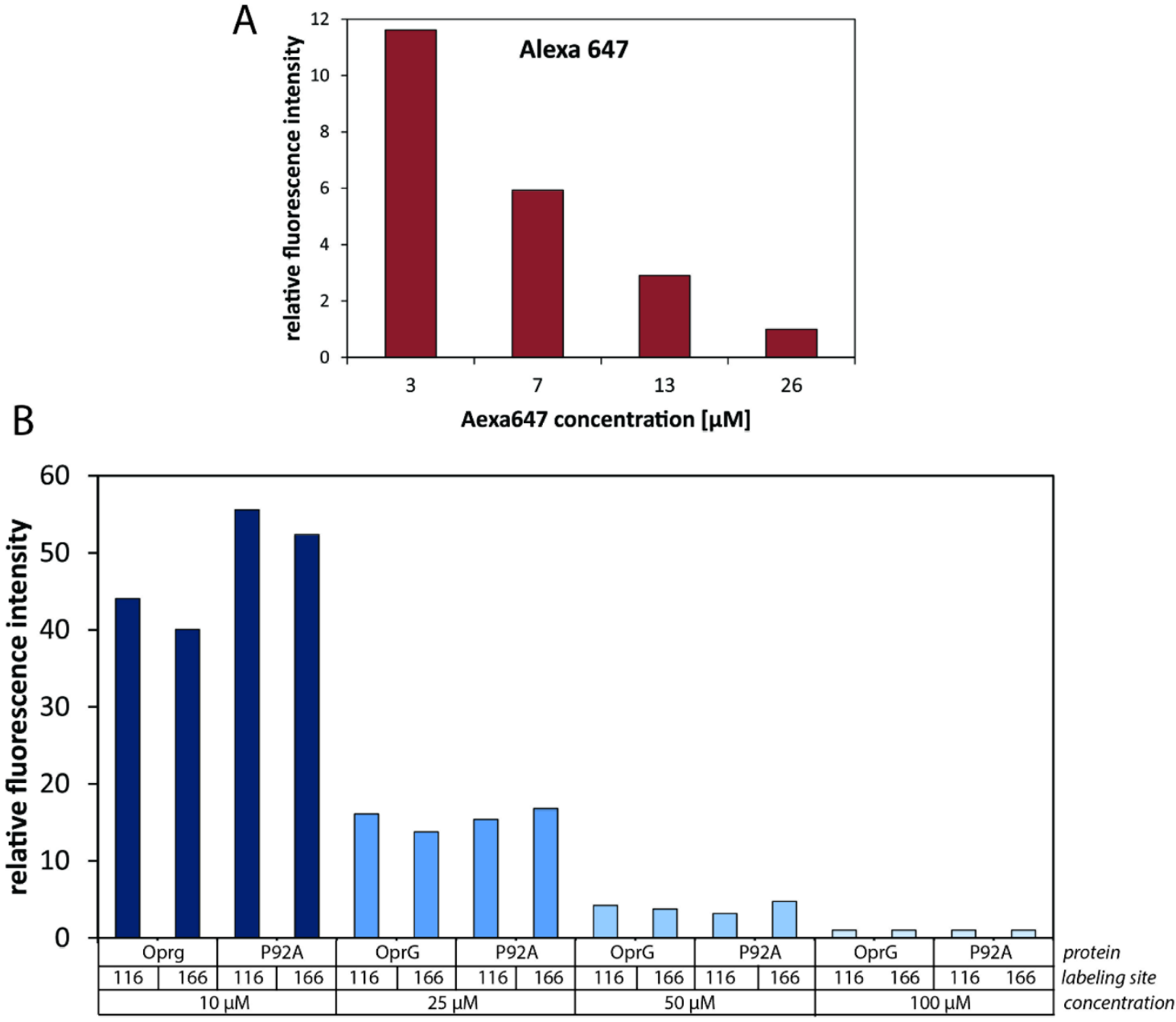

Fig. A1.2 Scaled fluorescence intensities of free Alexa647 dye (A) and Alexa647-labeled wt and P92A OprG in DHPC lipid micelles (B). 

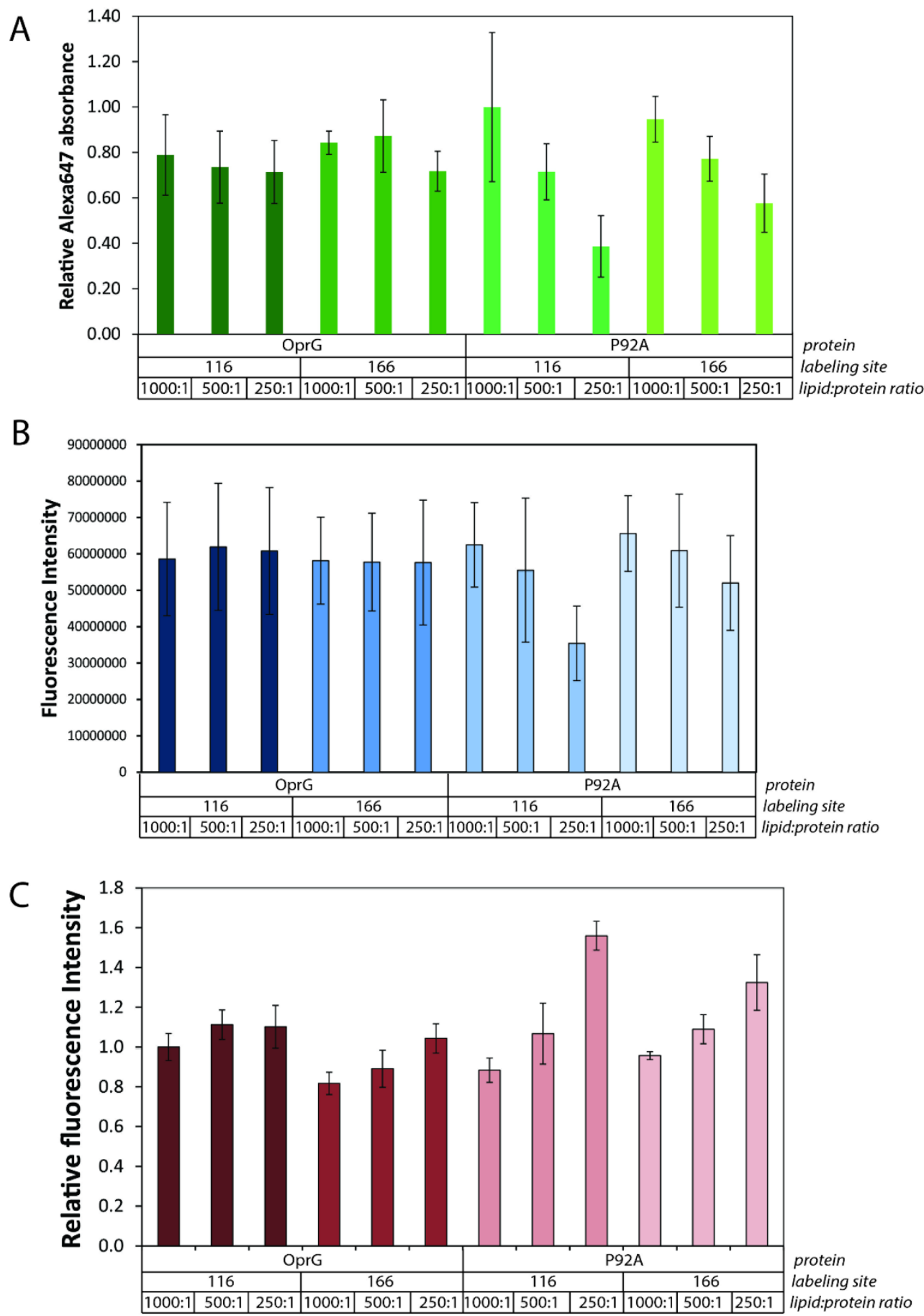

Fig. A1.3. A) Scaled absorbance of Alexa647-labeled OprG and P92A samples in POPC liposomes, established using the manufacturer's extinction coefficients. The means and standard deviations from at least three independent reconstitutions for each condition are shown. Raw (B) and scaled (C) fluorescence intensities of Alexa647labeled OprG and P92A in POPC lipid bilayers of different protein: lipid ratios. The means and standard deviations from at least three independent reconstitutions for 
To investigate the possible oligomerization of OprG and P92A in lipid bilayers, Alexa647labeled wt and P92A OprG were reconstituted into POPC liposomes at total protein:lipid ratios of 1:1000, 1: 500, and 1:250. The amount of protein reconstituted into liposomes were assessed by measuring the absorbance of Alexa647 using the manufacturer's extinction coefficients (Fig. A1.3 A). In the case of labeled OprG and P92A, the decrease in lipid:protein ratio (from 1000:1 to 250:1) lead to a decrease in the total amount of protein that was reconstituted in the liposomes. This is opposite to the effect that was observed with unlabeled proteins in liposomes (Table A1.1). This different behavior might be due to the presence of the label interfering with the reconstitution of the protein into lipid bilayer. Despite the lower amounts of protein being reconstituted into liposomes at 250:1 and 500:1 lipid:protein ratios, the relative fluorescence per protein molecule increases (Fig. A1.3 C). The most straightforward explanation of this effect is selfquenching of multimerizing OprG. This effect is weaker for wt OprG ( $21 \%$ increase in relative fluorescence from 1000:1 to 250:1 lipid: protein ratio), but becomes more significant for P92A OprG mutant (44\% increase in relative fluorescence from 1000:1 to 250:1 lipid:protein ratio), especially when this protein is labelled at 116 position.

\section{A1.4. Discussion}

Native PAGE experiments showed that wt OprG and its proline mutants (P66A, P91A, P92A) are present in DHPC detergent solution as a mixture of monomers, dimers, trimers and higher order oligomers. To verify that this tendency to multimerize is not only the result of the presence of DHPC in the protein solution, I attempted to reconstitute wt 
OprG and its proline mutants into liposomes of several different lipid compositions. However, P66A and P91A could not be effectively reconstituted into any liposomes tested. Wt and P92A OprG were successfully reconstituted into DMPC, POPC, and POPG/POPC liposomes, with efficiencies depending on the lipid composition of the bilayer. The reconstitution efficiency was lower for P92A than for the OprG under all the conditions tested. Native PAGE of wt and P92A OprG in POPC liposomes showed the presence of high molecular weight species, that might be protein or protein-lipid aggregates. To obtain more information about the oligomeric states of wt and P92A OprG, I fluorescently labelled both proteins with Alexa647. Quenching experiments in DHPC detergent and POPC liposome showed a weak propensity of wt OprG to multimerize and a slightly higher propensity for P92A OprG to multimerize. In summary, the obtained biophysical data suggest that introducing a proline mutation in the position 92 increases the tendency of the protein to oligomerize and partially interferes with the incorporation of the protein into lipid bilayers. However, further experiments are necessary to elucidate the exact oligomeric state of OprG in detergent micelles and in lipid bilayers. 


\section{References}

1. Silby, M.W., et al., Pseudomonas genomes: diverse and adaptable. FEMS Microbiol Rev, 2011. 35(4): p. 652-80.

2. Hancock, R.E. and F.S. Brinkman, Function of pseudomonas porins in uptake and efflux. Annu Rev Microbiol, 2002. 56: p. 17-38.

3. Stover, C.K., et al., Complete genome sequence of Pseudomonas aeruginosa PAO1, an opportunistic pathogen. Nature, 2000. 406(6799): p. 959-64.

4. Gellatly, S.L. and R.E. Hancock, Pseudomonas aeruginosa: new insights into pathogenesis and host defenses. Pathog Dis, 2013. 67(3): p. 159-73.

5. Vincent, J.L., Nosocomial infections in adult intensive-care units. Lancet, 2003. 361(9374): p. 2068-77.

6. Oliver, A., A. Mena, and M.D. Macià, Evolution of Pseudomonas aeruginosa pathogenicity: from acute to chronic infections. Evolutionary Biology of Bacterial and Fungal Pathogens. 2008: ASM Press.

7. Hancock, R.E., Resistance mechanisms in Pseudomonas aeruginosa and other nonfermentative gram-negative bacteria. Clin Infect Dis, 1998. 27 Suppl 1: p. S93-9.

8. Nicas, T.I. and R.E. Hancock, Pseudomonas aeruginosa outer membrane permeability: isolation of a porin protein F-deficient mutant. J Bacteriol, 1983. 153(1): p. 281-5.

9. Breidenstein, E.B., C. de la Fuente-Nunez, and R.E. Hancock, Pseudomonas aeruginosa: all roads lead to resistance. Trends Microbiol, 2011. 19(8): p. 419-26.

10. Lister, P.D., D.J. Wolter, and N.D. Hanson, Antibacterial-resistant Pseudomonas aeruginosa: clinical impact and complex regulation of chromosomally encoded resistance mechanisms. Clin Microbiol Rev, 2009. 22(4): p. 582-610.

11. Livermore, D.M., Multiple mechanisms of antimicrobial resistance in Pseudomonas aeruginosa: our worst nightmare? Clin Infect Dis, 2002. 34(5): p. 634-40.

12. Poole, K., Pseudomonas aeruginosa: resistance to the max. Front Microbiol, 2011. 2: p. 65.

13. Koebnik, R., K.P. Locher, and P. Van Gelder, Structure and function of bacterial outer membrane proteins: barrels in a nutshell. Mol Microbiol, 2000. 37(2): p. 239-53.

14. Mayer, H., R.N. Tharanathan, and J. Weckesse, Analysis of Lipopolysaccharides of GramNegative Bacteria. Methods in Microbiology. Vol. 18. 1985.

15. Schwechheimer, C. and M.J. Kuehn, Outer-membrane vesicles from Gram-negative bacteria: biogenesis and functions. Nat Rev Microbiol, 2015. 13(10): p. 605-19.

16. Qiao, S., et al., Structural basis for lipopolysaccharide insertion in the bacterial outer membrane. Nature, 2014. 511(7507): p. 108-11.

17. Pier, G.B., Pseudomonas aeruginosa lipopolysaccharide: a major virulence factor, initiator of inflammation and target for effective immunity. Int J Med Microbiol, 2007. 297(5): p. 277-95.

18. Ernst, R.K., et al., Pseudomonas aeruginosa lipid A diversity and its recognition by Toll-like receptor 4. J Endotoxin Res, 2003. 9(6): p. 395-400.

19. Ernst, R.K., et al., Specific lipopolysaccharide found in cystic fibrosis airway Pseudomonas aeruginosa. Science, 1999. 286(5444): p. 1561-5. 
20. Bystrova, O.V., et al., Structures of the core oligosaccharide and O-units in the R-and SRtype lipopolysaccharides of reference strains of Pseudomonas aeruginosa O-serogroups. FEMS Immunol Med Microbiol, 2006. 46(1): p. 85-99.

21. Bystrova, O.V., et al., Elucidation of the structure of the lipopolysaccharide core and the linkage between the core and the O-antigen in Pseudomonas aeruginosa immunotype 5 using strong alkaline degradation of the lipopolysaccharide. Biochemistry (Mosc), 2003. 68(8): p. 918-25.

22. Beckmann, F., et al., Preliminary communication 7-O-carbamoyl-L-glycero-D-mannoheptose: a new core constituent in the lipopolysaccharide of Pseudomonas aeruginosa. Carbohydr Res, 1995. 267(2): p. C3-7.

23. Knirel, Y.A., J.H. Helbig, and U. Zahringer, Structure of a decasaccharide isolated by mild acid degradation and dephosphorylation of the lipopolysaccharide of Pseudomonas fluorescens strain ATCC 49271. Carbohydr Res, 1996. 283: p. 129-39.

24. Bystrova, O.V., et al., Structural studies on the core and the O-polysaccharide repeating unit of Pseudomonas aeruginosa immunotype 1 lipopolysaccharide. Eur J Biochem, 2002. 269(8): p. 2194-203.

25. Knirel, Y.A., et al., Structural analysis of the lipopolysaccharide core of a rough, cystic fibrosis isolate of Pseudomonas aeruginosa. Eur J Biochem, 2001. 268(17): p. 4708-19.

26. Sadovskaya, I., et al., Structural characterization of the outer core and the O-chain linkage region of lipopolysaccharide from Pseudomonas aeruginosa serotype 05. Eur J Biochem, 2000. 267(6): p. 1640-50.

27. Walsh, A.G., et al., Lipopolysaccharide core phosphates are required for viability and intrinsic drug resistance in Pseudomonas aeruginosa. Mol Microbiol, 2000. 35(4): p. 71827.

28. Abeyrathne, P.D., et al., Functional characterization of WaaL, a ligase associated with linking O-antigen polysaccharide to the core of Pseudomonas aeruginosa lipopolysaccharide. J Bacteriol, 2005. 187(9): p. 3002-12.

29. Evans, D.J., et al., The rfb locus from Pseudomonas aeruginosa strain PA103 promotes the expression of $O$ antigen by both LPS-rough and LPS-smooth isolates from cystic fibrosis patients. Mol Microbiol, 1994. 13(3): p. 427-34.

30. Leive, L. and D.C. Morrison, Isolation of Lipopolysaccharides from Bacteria. Methods in Enzymology. Vol. XXVII. 1972, New York: Academic Press.

31. Jann, B., K. Reske, and K. Jann, Heterogeneity of lipopolysaccharides. Analysis of polysaccharide chain lengths by sodium dodecylsulfate-polyacrylamide gel electrophoresis. Eur J Biochem, 1975. 60(1): p. 239-46.

32. Brandenburg, K., et al., Supramolecular structure of enterobacterial wild-type lipopolysaccharides (LPS), fractions thereof, and their neutralization by Pep19-2.5. J Struct Biol, 2016. 194(1): p. 68-77.

33. Schmidtchen, A. and M. Malmsten, Peptide interactions with bacterial lipopolysaccharides. Current Opinion in Colloid \& Interface Science, 2013. 18 (2013) p. 381-392.

34. Brogden, K.A., Antimicrobial peptides: pore formers or metabolic inhibitors in bacteria? Nat Rev Microbiol, 2005. 3(3): p. 238-50.

35. Elsbach, P., What is the real role of antimicrobial polypeptides that can mediate several other inflammatory responses? J Clin Invest, 2003. 111(11): p. 1643-5. 
36. Ganz, T., Defensins: antimicrobial peptides of innate immunity. Nat Rev Immunol, 2003. 3(9): p. 710-20.

37. Hancock, R.E. and H.G. Sahl, Antimicrobial and host-defense peptides as new anti-infective therapeutic strategies. Nat Biotechnol, 2006. 24(12): p. 1551-7.

38. Strömstedt, A.A., et al., Interaction between amphiphilic peptides and phospholipid membranes. Curr Opin Colloid Interface Sci, 2010. 15: p. 467-78.

39. David, S.A., Towards a rational development of anti-endotoxin agents: novel approaches to sequestration of bacterial endotoxins with small molecules. J Mol Recognit, 2001. 14(6): p. 370-87.

40. Ding, L., et al., Interaction of antimicrobial peptides with lipopolysaccharides. Biochemistry, 2003. 42(42): p. 12251-9.

41. Mares, J., et al., Interactions of lipopolysaccharide and polymyxin studied by NMR spectroscopy. J Biol Chem, 2009. 284(17): p. 11498-506.

42. Pristovsek, P. and J. Kidric, Solution structure of polymyxins $B$ and $E$ and effect of binding to lipopolysaccharide: an NMR and molecular modeling study. J Med Chem, 1999. 42(22): p. 4604-13.

43. Andra, J., et al., Enhancement of endotoxin neutralization by coupling of a C12-alkyl chain to a lactoferricin-derived peptide. Biochem J, 2005. 385(Pt 1): p. 135-43.

44. Bhattacharjya, S., et al., High-resolution solution structure of a designed peptide bound to lipopolysaccharide: transferred nuclear Overhauser effects, micelle selectivity, and antiendotoxic activity. Biochemistry, 2007. 46(20): p. 5864-74.

45. Bhunia, A., P.N. Domadia, and S. Bhattacharjya, Structural and thermodynamic analyses of the interaction between melittin and lipopolysaccharide. Biochim Biophys Acta, 2007. 1768(12): p. 3282-91.

46. Papo, N. and Y. Shai, A molecular mechanism for lipopolysaccharide protection of Gramnegative bacteria from antimicrobial peptides. J Biol Chem, 2005. 280(11): p. 10378-87.

47. Pristovsek, P., et al., Structure of a synthetic fragment of the LALF protein when bound to lipopolysaccharide. J Med Chem, 2005. 48(5): p. 1666-70.

48. Rana, F.R. and J. Blazyk, Interactions between the antimicrobial peptide, magainin 2, and Salmonella typhimurium lipopolysaccharides. FEBS Lett, 1991. 293(1-2): p. 11-5.

49. Rosenfeld, Y., N. Papo, and Y. Shai, Endotoxin (lipopolysaccharide) neutralization by innate immunity host-defense peptides. Peptide properties and plausible modes of action. J Biol Chem, 2006. 281(3): p. 1636-43.

50. Gruber, A., et al., Structural model of MD-2 and functional role of its basic amino acid clusters involved in cellular lipopolysaccharide recognition. J Biol Chem, 2004. 279(27): p. 28475-82.

51. Ferguson, A.D., et al., Siderophore-mediated iron transport: crystal structure of FhuA with bound lipopolysaccharide. Science, 1998. 282(5397): p. 2215-20.

52. Ferguson, A.D., et al., A conserved structural motif for lipopolysaccharide recognition by procaryotic and eucaryotic proteins. Structure, 2000. 8(6): p. 585-92.

53. Zavascki, A.P., et al., Polymyxin B for the treatment of multidrug-resistant pathogens: $a$ critical review. J Antimicrob Chemother, 2007. 60(6): p. 1206-15.

54. Medzhitov, R., P. Preston-Hurlburt, and C.A. Janeway, Jr., A human homologue of the Drosophila Toll protein signals activation of adaptive immunity. Nature, 1997. 388(6640): p. 394-7.

55. Fitzgerald, K.A. and Z.J. Chen, Sorting out Toll signals. Cell, 2006. 125(5): p. 834-6. 
56. Schnare, M., M. Rollinghoff, and S. Qureshi, Toll-like receptors: sentinels of host defence against bacterial infection. Int Arch Allergy Immunol, 2006. 139(1): p. 75-85.

57. Zweigner, J., R.R. Schumann, and J.R. Weber, The role of lipopolysaccharide-binding protein in modulating the innate immune response. Microbes Infect, 2006. 8(3): p. 94652.

58. Blander, J.M. and R. Medzhitov, Toll-dependent selection of microbial antigens for presentation by dendritic cells. Nature, 2006. 440(7085): p. 808-12.

59. Pier, G.B., R.B. Markham, and D. Eardley, Correlation of the biologic responses of C3H/HEJ mice to endotoxin with the chemical and structural properties of the lipopolysaccharides from Pseudomonas aeruginosa and Escherichia coli. J Immunol, 1981. 127(1): p. 184-91.

60. Blattner, F.R., et al., The complete genome sequence of Escherichia coli $K-12$. Science, 1997. 277(5331): p. 1453-62.

61. Nikaido, H., Preventing drug access to targets: cell surface permeability barriers and active efflux in bacteria. Semin Cell Dev Biol, 2001. 12(3): p. 215-23.

62. Brenner, S.E. and M. Levitt, Expectations from structural genomics. Protein Sci, 2000. 9(1): p. 197-200.

63. Rosenbusch, J.P., Secondary and tertiary structure of membrane proteins. Zbl Bakt. Vol. 17. 1988.

64. Murzin, A.G., A.M. Lesk, and C. Chothia, Principles determining the structure of beta-sheet barrels in proteins. I. A theoretical analysis. J Mol Biol, 1994. 236(5): p. 1369-81.

65. Liu, W.M., Shear numbers of protein beta-barrels: definition refinements and statistics. J Mol Biol, 1998. 275(4): p. 541-5.

66. Schulz, G.E. and R.H. Schirmer, Principles of protein structure. 1979: Springer-Verlag.

67. Schulz, G.E., The structure of bacterial outer membrane proteins. Biochim Biophys Acta, 2002. 1565(2): p. 308-17.

68. Nikaido, H., Porins and specific diffusion channels in bacterial outer membranes. J Biol Chem, 1994. 269(6): p. 3905-8.

69. Nikaido, H., Molecular basis of bacterial outer membrane permeability revisited. Microbiol Mol Biol Rev, 2003. 67(4): p. 593-656.

70. Eren, E., et al., Substrate specificity within a family of outer membrane carboxylate channels. PLoS Biol, 2012. 10(1): p. e1001242.

71. Yoshimura, F. and H. Nikaido, Permeability of Pseudomonas aeruginosa outer membrane to hydrophilic solutes. J Bacteriol, 1982. 152(2): p. 636-42.

72. Nikaido, H. and R.E. Hancock, Outer membrane permeability of Pseudomonas aeruginosa. The Bacteria: A treatise on structure and function. Vol. X. 1986, London: Academic Press.

73. McPhee, J.B., et al., The major outer membrane protein OprG of Pseudomonas aeruginosa contributes to cytotoxicity and forms an anaerobically regulated, cation-selective channel. FEMS Microbiol Lett, 2009. 296(2): p. 241-7.

74. Touw, D.S., D.R. Patel, and B. van den Berg, The crystal structure of OprG from Pseudomonas aeruginosa, a potential channel for transport of hydrophobic molecules across the outer membrane. PLoS One, 2010. 5(11): p. e15016.

75. Das, M., et al., Antisera to selected outer membrane proteins of Vibrio cholerae protect against challenge with homologous and heterologous strains of $V$. cholerae. FEMS Immunol Med Microbiol, 1998. 22(4): p. 303-8. 
76. Jalajakumari, M.B. and P.A. Manning, Nucleotide sequence of the gene, ompW, encoding a $22 \mathrm{kDa}$ immunogenic outer membrane protein of Vibrio cholerae. Nucleic Acids Res, 1990. 18(8): p. 2180.

77. Soderblom, T., et al., Effects of the Escherichia coli toxin cytolysin A on mucosal immunostimulation via epithelial Ca2+ signalling and Toll-like receptor 4. Cell Microbiol, 2005. 7(6): p. 779-88.

78. Arnold, T., K. Zeth, and D. Linke, Structure and function of colicin S4, a colicin with a duplicated receptor-binding domain. J Biol Chem, 2009. 284(10): p. 6403-13.

79. $\mathrm{Wu}$, L., et al., $\mathrm{OmpW}$ and $\mathrm{OmpV}$ are required for $\mathrm{NaCl}$ regulation in Photobacterium damsela. J Proteome Res, 2006. 5(9): p. 2250-7.

80. Nandi, B., et al., Structural features, properties and regulation of the outer-membrane protein W (OmpW) of Vibrio cholerae. Microbiology, 2005. 151(Pt 9): p. 2975-86.

81. Neher, T.M. and D.R. Lueking, Pseudomonas fluorescens ompW: plasmid localization and requirement for naphthalene uptake. Can J Microbiol, 2009. 55(5): p. 553-63.

82. Yates, J.M., G. Morris, and M.R. Brown, Effect of iron concentration and growth rate on the expression of protein $G$ in Pseudomonas aeruginosa. FEMS Microbiol Lett, 1989. 49(23): p. 259-62.

83. Chamberland, S., et al., Characterization of mechanisms of quinolone resistance in Pseudomonas aeruginosa strains isolated in vitro and in vivo during experimental endocarditis. Antimicrob Agents Chemother, 1989. 33(5): p. 624-34.

84. Peng, X., et al., Proteomic analysis of the sarcosine-insoluble outer membrane fraction of Pseudomonas aeruginosa responding to ampicilin, kanamycin, and tetracycline resistance. J Proteome Res, 2005. 4(6): p. 2257-65.

85. Hong, H., et al., The outer membrane protein OmpW forms an eight-stranded beta-barrel with a hydrophobic channel. J Biol Chem, 2006. 281(11): p. 7568-77.

86. Hearn, E.M., et al., Transmembrane passage of hydrophobic compounds through a protein channel wall. Nature, 2009. 458(7236): p. 367-70.

87. van den Berg, B., The FadL family: unusual transporters for unusual substrates. Curr Opin Struct Biol, 2005. 15(4): p. 401-7.

88. Denome, S.A., et al., Metabolism of dibenzothiophene and naphthalene in Pseudomonas strains: complete DNA sequence of an upper naphthalene catabolic pathway. J Bacteriol, 1993. 175(21): p. 6890-901.

89. Eaton, R.W., Organization and evolution of naphthalene catabolic pathways: sequence of the DNA encoding 2-hydroxychromene-2-carboxylate isomerase and trans-ohydroxybenzylidenepyruvate hydratase-aldolase from the NAH7 plasmid. J Bacteriol, 1994. 176(24): p. 7757-62.

90. van Beilen, J.B., et al., DNA sequence determination and functional characterization of the OCT-plasmid-encoded alkJKL genes of Pseudomonas oleovorans. Mol Microbiol, 1992. 6(21): p. 3121-36.

91. Rehm, B.H. and R.E. Hancock, Membrane topology of the outer membrane protein OprH from Pseudomonas aeruginosa: PCR-mediated site-directed insertion and deletion mutagenesis. J Bacteriol, 1996. 178(11): p. 3346-9.

92. Edrington, T.C., et al., Structural basis for the interaction of lipopolysaccharide with outer membrane protein $\mathrm{H}(\mathrm{OprH})$ from Pseudomonas aeruginosa. J Biol Chem, 2011. 286(45): p. 39211-23. 
93. Macfarlane, E.L., A. Kwasnicka, and R.E. Hancock, Role of Pseudomonas aeruginosa PhoPpho $Q$ in resistance to antimicrobial cationic peptides and aminoglycosides. Microbiology, 2000. 146 ( Pt 10): p. 2543-54.

94. Soncini, F.C. and E.A. Groisman, Two-component regulatory systems can interact to process multiple environmental signals. J Bacteriol, 1996. 178(23): p. 6796-801.

95. McPhee, J.B., S. Lewenza, and R.E. Hancock, Cationic antimicrobial peptides activate a two-component regulatory system, PmrA-PmrB, that regulates resistance to polymyxin $B$ and cationic antimicrobial peptides in Pseudomonas aeruginosa. Mol Microbiol, 2003. 50(1): p. 205-17.

96. Macfarlane, E.L., et al., PhoP-PhoQ homologues in Pseudomonas aeruginosa regulate expression of the outer-membrane protein $\mathrm{OprH}$ and polymyxin $B$ resistance. Mol Microbiol, 1999. 34(2): p. 305-16.

97. Gellatly, S.L., et al., The Pseudomonas aeruginosa PhoP-PhoQ two-component regulatory system is induced upon interaction with epithelial cells and controls cytotoxicity and inflammation. Infect Immun, 2012. 80(9): p. 3122-31.

98. Fothergill, J.L., C. Winstanley, and C.E. James, Novel therapeutic strategies to counter Pseudomonas aeruginosa infections. Expert Rev Anti Infect Ther, 2012. 10(2): p. 219-35.

99. Bisbe, J., et al., Pseudomonas aeruginosa bacteremia: univariate and multivariate analyses of factors influencing the prognosis in 133 episodes. Rev Infect Dis, 1988. 10(3): p. 629-35.

100. Vidal, F., et al., Epidemiology and outcome of Pseudomonas aeruginosa bacteremia, with special emphasis on the influence of antibiotic treatment. Analysis of 189 episodes. Arch Intern Med, 1996. 156(18): p. 2121-6.

101. Peng, X., Ren, Lin, Wu, Proteomic Analysis of the Sarcosine-Insoluble Outer Membrane Fraction of Pseudomonas aeruginosa Responding to Ampicilin, Kanamycin, and Tetracycline Resistance. 2005.

102. Govan, J.R., A.R. Brown, and A.M. Jones, Evolving epidemiology of Pseudomonas aeruginosa and the Burkholderia cepacia complex in cystic fibrosis lung infection. Future Microbiol, 2007. 2(2): p. 153-64.

103. Rajan, S. and L. Saiman, Pulmonary infections in patients with cystic fibrosis. Semin Respir Infect, 2002. 17(1): p. 47-56.

104. Delcour, A.H., Outer membrane permeability and antibiotic resistance. Biochim Biophys Acta, 2009. 1794(5): p. 808-16.

105. Tamber, S., M.M. Ochs, and R.E. Hancock, Role of the novel OprD family of porins in nutrient uptake in Pseudomonas aeruginosa. J Bacteriol, 2006. 188(1): p. 45-54.

106. Mesaros, N., et al., Pseudomonas aeruginosa: resistance and therapeutic options at the turn of the new millennium. Clin Microbiol Infect, 2007. 13(6): p. 560-78.

107. Walzer, G., E. Rosenberg, and E.Z. Ron, Identification of outer membrane proteins with emulsifying activity by prediction of beta-barrel regions. J Microbiol Methods, 2009. 76(1): p. 52-7.

108. Waugh, D.S., Genetic tools for selective labeling of proteins with alpha-15N-amino acids. J Biomol NMR, 1996. 8(2): p. 184-92.

109. Nikaido, H. and E.Y. Rosenberg, Porin channels in Escherichia coli: studies with liposomes reconstituted from purified proteins. J Bacteriol, 1983. 153(1): p. 241-52. 
110. Bangham, A.D., J. De Gier, and G.D. Greville, Osmotic properties and water permeability of phospholipid liquid crystals. Chemistry and Physics of Lipids 1 1967. 5/1967: p. 225246.

111. Goddard, T.D. and D.G. Kneller, SPARKY 3. University of California, San Francisco.

112. Shen, Y., et al., TALOS+: a hybrid method for predicting protein backbone torsion angles from NMR chemical shifts. J Biomol NMR, 2009. 44(4): p. 213-23.

113. Brunger, A.T., et al., Crystallography \& NMR system: A new software suite for macromolecular structure determination. Acta Crystallogr D Biol Crystallogr, 1998. 54(Pt 5): p. 905-21.

114. Laskowski, R.A., et al., AQUA and PROCHECK-NMR: programs for checking the quality of protein structures solved by NMR. J Biomol NMR, 1996. 8(4): p. 477-86.

115. Johnson, D.A., et al., High-throughput phenotypic characterization of Pseudomonas aeruginosa membrane transport genes. PLoS Genet, 2008. 4(10): p. e1000211.

116. Metzler, W.J., et al., Characterization of the three-dimensional solution structure of human profilin: $1 \mathrm{H}, 13 \mathrm{C}$, and $15 \mathrm{~N} \mathrm{NMR}$ assignments and global folding pattern. Biochemistry, 1993. 32(50): p. 13818-29.

117. Cornilescu, G., F. Delaglio, and A. Bax, Protein backbone angle restraints from searching a database for chemical shift and sequence homology. J Biomol NMR, 1999. 13(3): p. 289302.

118. Hong, H., et al., Role of aromatic side chains in the folding and thermodynamic stability of integral membrane proteins. J Am Chem Soc, 2007. 129(26): p. 8320-7.

119. Lee, D., et al., Effective rotational correlation times of proteins from NMR relaxation interference. J Magn Reson, 2006. 178(1): p. 72-6.

120. Lathe, W.C., 3rd, B. Snel, and P. Bork, Gene context conservation of a higher order than operons. Trends Biochem Sci, 2000. 25(10): p. 474-9.

121. Wu, E.L., et al., E. coli outer membrane and interactions with OmpLA. Biophys J, 2014. 106(11): p. 2493-502.

122. Cierpicki, T., et al., Increasing the accuracy of solution NMR structures of membrane proteins by application of residual dipolar couplings. High-resolution structure of outer membrane protein A. J Am Chem Soc, 2006. 128(21): p. 6947-51.

123. Pautsch, A. and G.E. Schulz, High-resolution structure of the OmpA membrane domain. J Mol Biol, 2000. 298(2): p. 273-82.

124. Liang, B. and L.K. Tamm, Structure of outer membrane protein $G$ by solution NMR spectroscopy. Proc Natl Acad Sci U S A, 2007. 104(41): p. 16140-5.

125. Yildiz, O., et al., Structure of the monomeric outer-membrane porin OmpG in the open and closed conformation. EMBO J, 2006. 25(15): p. 3702-13.

126. Fernandez, C., et al., NMR structure of the integral membrane protein OmpX. J Mol Biol, 2004. 336(5): p. 1211-21.

127. Vogt, J. and G.E. Schulz, The structure of the outer membrane protein OmpX from Escherichia coli reveals possible mechanisms of virulence. Structure, 1999. 7(10): p. 13019.

128. Lomize, A.L., et al., Positioning of proteins in membranes: a computational approach. Protein Sci, 2006. 15(6): p. 1318-33.

129. Hagn, F., et al., Optimized phospholipid bilayer nanodiscs facilitate high-resolution structure determination of membrane proteins. J Am Chem Soc, 2013. 135(5): p. 1919-25. 
130. Horst, R., P. Stanczak, and K. Wuthrich, NMR polypeptide backbone conformation of the E. coli outer membrane protein W. Structure, 2014. 22(8): p. 1204-9.

131. Kucharska, I., et al., Optimizing nanodiscs and bicelles for solution NMR studies of two beta-barrel membrane proteins. J Biomol NMR, 2015. 61(3-4): p. 261-74.

132. Fox, D.A., et al., Structure of the Neisserial outer membrane protein Opa(6)(0): loop flexibility essential to receptor recognition and bacterial engulfment. J Am Chem Soc, 2014. 136(28): p. 9938-46.

133. Zhuang, T., et al., NMR-based conformational ensembles explain $\mathrm{pH}$-gated opening and closing of OmpG channel. J Am Chem Soc, 2013. 135(40): p. 15101-13.

134. Vaara, M., Agents that increase the permeability of the outer membrane. Microbiol Rev, 1992. 56(3): p. 395-411.

135. Miller, A.K., et al., PhoQ mutations promote lipid A modification and polymyxin resistance of Pseudomonas aeruginosa found in colistin-treated cystic fibrosis patients. Antimicrob Agents Chemother, 2011. 55(12): p. 5761-9.

136. Mueller, P., et al., Reconstitution of cell membrane structure in vitro and its transformation into an excitable system. Nature, 1962. 194: p. 979-80.

137. Muller, C., P. Plesiat, and K. Jeannot, A two-component regulatory system interconnects resistance to polymyxins, aminoglycosides, fluoroquinolones, and beta-lactams in Pseudomonas aeruginosa. Antimicrob Agents Chemother, 2011. 55(3): p. 1211-21.

138. Tugarinov, V., V. Kanelis, and L.E. Kay, Isotope labeling strategies for the study of highmolecular-weight proteins by solution NMR spectroscopy. Nat Protoc, 2006. 1(2): p. 74954.

139. Kucharska, I., et al., OprG Harnesses the Dynamics of its Extracellular Loops to Transport Small Amino Acids across the Outer Membrane of Pseudomonas aeruginosa. Structure, 2015. 23(12): p. 2234-45.

140. Sturiale, L., et al., New conditions for matrix-assisted laser desorption/ionization mass spectrometry of native bacterial R-type lipopolysaccharides. Rapid Commun Mass Spectrom, 2005. 19(13): p. 1829-34.

141. Hardy, E., et al., Sensitive reverse staining of bacterial lipopolysaccharides on polyacrylamide gels by using zinc and imidazole salts. Anal Biochem, 1997. 244(1): p. 2832.

142. Hyberts, S.G., H. Arthanari, and G. Wagner, Applications of non-uniform sampling and processing. Top Curr Chem, 2012. 316: p. 125-48.

143. Hyberts, S.G., et al., Application of iterative soft thresholding for fast reconstruction of NMR data non-uniformly sampled with multidimensional Poisson Gap scheduling. J Biomol NMR, 2012. 52(4): p. 315-27.

144. Hyberts, S.G., K. Takeuchi, and G. Wagner, Poisson-gap sampling and forward maximum entropy reconstruction for enhancing the resolution and sensitivity of protein NMR data. J Am Chem Soc, 2010. 132(7): p. 2145-7.

145. Tobias, P.S., K. Soldau, and R.J. Ulevitch, Identification of a lipid A binding site in the acute phase reactant lipopolysaccharide binding protein. J Biol Chem, 1989. 264(18): p. 1086771.

146. Abraham, S.J., S. Hoheisel, and V. Gaponenko, Detection of protein-ligand interactions by NMR using reductive methylation of lysine residues. J Biomol NMR, 2008. 42(2): p. 143-8.

147. Means, G.E. and R.E. Feeney, Reductive alkylation of amino groups in proteins. Biochemistry, 1968. 7(6): p. 2192-201. 
148. Fielding, L., NMR methods for the determination of protein-ligand dissociation constants. Curr Top Med Chem, 2003. 3(1): p. 39-53.

149. Seelig, J., A. Seelig, and L.K. Tamm, Nuclear Magnetic Resonance and Lipid-Protein Interactions. . Lipid-Protein Interactions. Vol. 2. 1982, New York: John R. Wiley \& Sons.

150. Yeagle, P.L., Non-covalent binding of membrane lipids to membrane proteins. Biochim Biophys Acta, 2014. 1838(6): p. 1548-59.

151. Laganowsky, A., et al., Membrane proteins bind lipids selectively to modulate their structure and function. Nature, 2014. 510(7503): p. 172-5.

152. Srimal, S., et al., Titration calorimetric studies to elucidate the specificity of the interactions of polymyxin B with lipopolysaccharides and lipid A. Biochem J, 1996. 315 ( Pt 2): p. 679-86.

153. Schindler, M. and M.J. Osborn, Interaction of divalent cations and polymyxin $B$ with lipopolysaccharide. Biochemistry, 1979. 18(20): p. 4425-30.

154. Wallin, E. and G. von Heijne, Genome-wide analysis of integral membrane proteins from eubacterial, archaean, and eukaryotic organisms. Protein Sci, 1998. 7(4): p. 1029-38.

155. Linke, D., Detergents: an overview. Methods Enzymol, 2009. 463: p. 603-17.

156. Tate, C.G., Practical considerations of membrane protein instability during purification and crystallisation. Methods Mol Biol, 2010. 601: p. 187-203.

157. Sanders, C.R., 2nd and J.H. Prestegard, Magnetically orientable phospholipid bilayers containing small amounts of a bile salt analogue, CHAPSO. Biophys J, 1990. 58(2): p. 44760.

158. Sanders, C.R. and R.S. Prosser, Bicelles: a model membrane system for all seasons? Structure, 1998. 6(10): p. 1227-34.

159. Sanders, C.R., 2nd and J.P. Schwonek, Characterization of magnetically orientable bilayers in mixtures of dihexanoylphosphatidylcholine and dimyristoylphosphatidylcholine by solid-state NMR. Biochemistry, 1992. 31(37): p. 8898-905.

160. Lau, T.L., et al., Structure of the integrin beta3 transmembrane segment in phospholipid bicelles and detergent micelles. Biochemistry, 2008. 47(13): p. 4008-16.

161. Poget, S.F., S.M. Cahill, and M.E. Girvin, Isotropic bicelles stabilize the functional form of a small multidrug-resistance pump for NMR structural studies. J Am Chem Soc, 2007. 129(9): p. 2432-3.

162. Bocharov, E.V., et al., Spatial structure and $\mathrm{pH}$-dependent conformational diversity of dimeric transmembrane domain of the receptor tyrosine kinase EphA1. J Biol Chem, 2008. 283(43): p. 29385-95.

163. Liang, B., et al., The SNARE motif of synaptobrevin exhibits an aqueous-interfacial partitioning that is modulated by membrane curvature. Biochemistry, 2014. 53(9): p. 1485-94.

164. Song, Y., et al., Impact of bilayer lipid composition on the structure and topology of the transmembrane amyloid precursor C99 protein. J Am Chem Soc, 2014. 136(11): p. 40936.

165. Bayburt, T.H., Y.V. Grinkova, and S.G. Sligar, Single-molecule height measurements on microsomal cytochrome $\mathrm{P} 450$ in nanometer-scale phospholipid bilayer disks. Proc Natl Acad Sci U S A, 2002. 99(10): p. 6725-30.

166. Denisov, I.G., et al., Directed self-assembly of monodisperse phospholipid bilayer Nanodiscs with controlled size. J Am Chem Soc, 2004. 126(11): p. 3477-87. 
167. Gluck, J.M., et al., Integral membrane proteins in nanodiscs can be studied by solution NMR spectroscopy. J Am Chem Soc, 2009. 131(34): p. 12060-1.

168. Park, S.H., et al., Nanodiscs versus macrodiscs for NMR of membrane proteins. Biochemistry, 2011. 50(42): p. 8983-5.

169. Etzkorn, M., et al., Cell-free expressed bacteriorhodopsin in different soluble membrane mimetics: biophysical properties and NMR accessibility. Structure, 2013. 21(3): p. 394401.

170. Tzitzilonis, C., et al., Detergent/nanodisc screening for high-resolution NMR studies of an integral membrane protein containing a cytoplasmic domain. PLoS One, 2013. 8(1): p. e54378.

171. Susac, L., R. Horst, and K. Wuthrich, Solution-NMR characterization of outer-membrane protein A from E. coli in lipid bilayer nanodiscs and detergent micelles. Chembiochem, 2014. 15(7): p. 995-1000.

172. Raschle, T., et al., Structural and functional characterization of the integral membrane protein VDAC-1 in lipid bilayer nanodiscs. J Am Chem Soc, 2009. 131(49): p. 17777-9.

173. Yu, T.Y., et al., Solution NMR spectroscopic characterization of human VDAC-2 in detergent micelles and lipid bilayer nanodiscs. Biochim Biophys Acta, 2012. 1818(6): p. 1562-9.

174. Tropea, J.E., S. Cherry, and D.S. Waugh, Expression and purification of soluble His(6)tagged TEV protease. Methods Mol Biol, 2009. 498: p. 297-307.

175. Ritchie, T.K., et al., Chapter 11 - Reconstitution of membrane proteins in phospholipid bilayer nanodiscs. Methods Enzymol, 2009. 464: p. 211-31.

176. Ellena, J.F., M.C. Burnitz, and D.S. Cafiso, Location of the myristoylated alanine-rich Ckinase substrate (MARCKS) effector domain in negatively charged phospholipid bicelles. Biophys J, 2003. 85(4): p. 2442-8.

177. Losonczi, J.A. and J.H. Prestegard, Improved dilute bicelle solutions for high-resolution NMR of biological macromolecules. J Biomol NMR, 1998. 12(3): p. 447-51.

178. Struppe, J., et al., $2 H N M R$ studies of a myristoylated peptide in neutral and acidic phospholipid bicelles. Biochemistry, 1998. 37(44): p. 15523-7.

179. Delaglio, F., et al., NMRPipe: a multidimensional spectral processing system based on UNIX pipes. J Biomol NMR, 1995. 6(3): p. 277-93.

180. Mulder, F.A., et al., Altered flexibility in the substrate-binding site of related native and engineered high-alkaline Bacillus subtilisins. J Mol Biol, 1999. 292(1): p. 111-23.

181. Schuler, M.A., I.G. Denisov, and S.G. Sligar, Nanodiscs as a new tool to examine lipidprotein interactions. Methods Mol Biol, 2013. 974: p. 415-33.

182. Hyberts, S.G., et al., Perspectives in magnetic resonance: NMR in the post-FFT era. J Magn Reson, 2014. 241: p. 60-73.

183. Hyberts, S.G., S.A. Robson, and G. Wagner, Exploring signal-to-noise ratio and sensitivity in non-uniformly sampled multi-dimensional NMR spectra. J Biomol NMR, 2013. 55(2): p. 167-78.

184. Rovnyak, D., et al., Accelerated acquisition of high resolution triple-resonance spectra using non-uniform sampling and maximum entropy reconstruction. J Magn Reson, 2004. 170(1): p. 15-21.

185. Murray, D.H. and L.K. Tamm, Clustering of syntaxin-1A in model membranes is modulated by phosphatidylinositol 4,5-bisphosphate and cholesterol. Biochemistry, 2009. 48(21): p. 4617-25. 